JOURNAL OF THE

AMERICAN MATHEMATICAL SOCIETY

Volume 14, Number 4, Pages 843-939

S 0894-0347(01)00370-8

Article electronically published on May 15, 2001

\title{
ON THE MODULARITY OF ELLIPTIC CURVES OVER Q: WILD 3-ADIC EXERCISES
}

CHRISTOPHE BREUIL, BRIAN CONRAD, FRED DIAMOND, AND RICHARD TAYLOR

\section{INTRODUCTION}

In this paper, building on work of Wiles [Wi] and of Taylor and Wiles [TW], we will prove the following two theorems (see $\$ 2.2$ ).

Theorem A. If $E_{/ \mathbf{Q}}$ is an elliptic curve, then $E$ is modular.

Theorem B. If $\bar{\rho}: \operatorname{Gal}(\overline{\mathbf{Q}} / \mathbf{Q}) \rightarrow \mathrm{GL}_{2}\left(\mathbf{F}_{5}\right)$ is an irreducible continuous representation with cyclotomic determinant, then $\bar{\rho}$ is modular.

We will first remind the reader of the content of these results and then briefly outline the method of proof.

If $N$ is a positive integer, then we let $\Gamma_{1}(N)$ denote the subgroup of $\mathrm{SL}_{2}(\mathbf{Z})$ consisting of matrices that modulo $N$ are of the form

$$
\left(\begin{array}{ll}
1 & * \\
0 & 1
\end{array}\right) \text {. }
$$

The quotient of the upper half plane by $\Gamma_{1}(N)$, acting by fractional linear transformations, is the complex manifold associated to an affine algebraic curve $Y_{1}(N) / \mathbf{C}$. This curve has a natural model $Y_{1}(N)_{/ \mathbf{Q}}$, which for $N>3$ is a fine moduli scheme for elliptic curves with a point of exact order $N$. We will let $X_{1}(N)$ denote the smooth projective curve which contains $Y_{1}(N)$ as a dense Zariski open subset.

Recall that a cusp form of weight $k \geq 1$ and level $N \geq 1$ is a holomorphic function $f$ on the upper half complex plane $\mathfrak{H}$ such that

- for all matrices

$$
\left(\begin{array}{ll}
a & b \\
c & d
\end{array}\right) \in \Gamma_{1}(N)
$$

and all $z \in \mathfrak{H}$, we have $f((a z+b) /(c z+d))=(c z+d)^{k} f(z)$;

- and $|f(z)|^{2}(\operatorname{Im} z)^{k}$ is bounded on $\mathfrak{H}$.

Received by the editors February 28, 2000 and, in revised form, January 1, 2001.

2000 Mathematics Subject Classification. Primary 11G05; Secondary 11F80.

Key words and phrases. Elliptic curve, Galois representation, modularity.

The first author was supported by the CNRS. The second author was partially supported by a grant from the NSF. The third author was partially supported by a grant from the NSF and an AMS Centennial Fellowship, and was working at Rutgers University during much of the research. The fourth author was partially supported by a grant from the NSF and by the Miller Institute for Basic Science. 
The space $S_{k}(N)$ of cusp forms of weight $k$ and level $N$ is a finite-dimensional complex vector space. If $f \in S_{k}(N)$, then it has an expansion

$$
f(z)=\sum_{n=1}^{\infty} c_{n}(f) e^{2 \pi i n z}
$$

and we define the $L$-series of $f$ to be

$$
L(f, s)=\sum_{n=1}^{\infty} c_{n}(f) / n^{s} .
$$

For each prime $p \nmid N$ there is a linear operator $T_{p}$ on $S_{k}(N)$ defined by

$$
\left(f \mid T_{p}\right)(z)=p^{-1} \sum_{i=0}^{p-1} f((z+i) / p)+p^{k-1}(c p z+d)^{-k} f((a p z+b) /(c p z+d))
$$

for any

$$
\left(\begin{array}{ll}
a & b \\
c & d
\end{array}\right) \in \mathrm{SL}_{2}(\mathbf{Z})
$$

with $c \equiv 0 \bmod N$ and $d \equiv p \bmod N$. The operators $T_{p}$ for $p \nmid N$ can be simultaneously diagonalised on the space $S_{k}(N)$ and a simultaneous eigenvector is called an eigenform. If $f$ is an eigenform, then the corresponding eigenvalues, $a_{p}(f)$, are algebraic integers and we have $c_{p}(f)=a_{p}(f) c_{1}(f)$.

Let $\lambda$ be a place of the algebraic closure of $\mathbf{Q}$ in $\mathbf{C}$ above a rational prime $\ell$ and let $\overline{\mathbf{Q}}_{\lambda}$ denote the algebraic closure of $\mathbf{Q}_{\ell}$ thought of as a $\overline{\mathbf{Q}}$ algebra via $\lambda$. If $f \in S_{k}(N)$ is an eigenform, then there is a unique continuous irreducible representation

$$
\rho_{f, \lambda}: \operatorname{Gal}(\overline{\mathbf{Q}} / \mathbf{Q}) \longrightarrow \mathrm{GL}_{2}\left(\overline{\mathbf{Q}}_{\lambda}\right)
$$

such that for any prime $p \nmid N l, \rho_{f, \lambda}$ is unramified at $p$ and $\operatorname{tr} \rho_{f, \lambda}\left(\operatorname{Frob}_{p}\right)=a_{p}(f)$. The existence of $\rho_{f, \lambda}$ is due to Shimura if $k=2$ [Sh2], to Deligne if $k>2$ [De] and to Deligne and Serre if $k=1$ [DS]. Its irreducibility is due to Ribet if $k>1$ Ri] and to Deligne and Serre if $k=1[\mathrm{DS}]$. Moreover $\rho$ is odd in the sense that $\operatorname{det} \rho$ of complex conjugation is -1 . Also, $\rho_{f, \lambda}$ is potentially semi-stable at $\ell$ in the sense of Fontaine. We can choose a conjugate of $\rho_{f, \lambda}$ which is valued in $\mathrm{GL}_{2}\left(\mathcal{O}_{\overline{\mathbf{Q}}_{\lambda}}\right)$, and reducing modulo the maximal ideal and semi-simplifying yields a continuous representation

$$
\bar{\rho}_{f, \lambda}: \operatorname{Gal}(\overline{\mathbf{Q}} / \mathbf{Q}) \longrightarrow \mathrm{GL}_{2}\left(\overline{\mathbf{F}}_{\ell}\right),
$$

which, up to isomorphism, does not depend on the choice of conjugate of $\rho_{f, \lambda}$.

Now suppose that $\rho: G_{\mathbf{Q}} \rightarrow \mathrm{GL}_{2}\left(\overline{\mathbf{Q}}_{\ell}\right)$ is a continuous representation which is unramified outside finitely many primes and for which the restriction of $\rho$ to a decomposition group at $\ell$ is potentially semi-stable in the sense of Fontaine. To $\left.\rho\right|_{\text {Gal }\left(\overline{\mathbf{Q}}_{\ell} / \mathbf{Q}_{\ell}\right)}$ we can associate both a pair of Hodge-Tate numbers and a Weil-Deligne representation of the Weil group of $\mathbf{Q}_{\ell}$. We define the conductor $N(\rho)$ of $\rho$ to be the product over $p \neq \ell$ of the conductor of $\left.\rho\right|_{\operatorname{Gal}\left(\overline{\mathbf{Q}}_{p} / \mathbf{Q}_{p}\right)}$ and of the conductor of the Weil-Deligne representation associated to $\left.\rho\right|_{\text {Gal }\left(\overline{\mathbf{Q}}_{\ell} / \mathbf{Q}_{\ell}\right)}$. We define the weight $k(\rho)$ of $\rho$ to be 1 plus the absolute difference of the two Hodge-Tate numbers of $\left.\rho\right|_{\operatorname{Gal}\left(\overline{\mathbf{Q}}_{\ell} / \mathbf{Q}_{\ell}\right)}$. It is known by work of Carayol and others that the following two 
conditions are equivalent:

- $\rho \sim \rho_{f, \lambda}$ for some eigenform $f$ and some place $\lambda \mid \ell$;

- $\rho \sim \rho_{f, \lambda}$ for some eigenform $f$ of level $N(\rho)$ and weight $k(\rho)$ and some place $\lambda \mid \ell$.

When these equivalent conditions are met we call $\rho$ modular. It is conjectured by Fontaine and Mazur that if $\rho: G_{\mathbf{Q}} \rightarrow \mathrm{GL}_{2}\left(\overline{\mathbf{Q}}_{\ell}\right)$ is a continuous irreducible representation which satisfies

- $\rho$ is unramified outside finitely many primes,

- $\left.\rho\right|_{\operatorname{Gal}\left(\overline{\mathbf{Q}}_{\ell} / \mathbf{Q}_{\ell}\right)}$ is potentially semi-stable with its smaller Hodge-Tate number 0 ,

- and, in the case where both Hodge-Tate numbers are zero, $\rho$ is odd,

then $\rho$ is modular $[\mathrm{FM}$.

Next consider a continuous irreducible representation $\bar{\rho}: \operatorname{Gal}(\overline{\mathbf{Q}} / \mathbf{Q}) \rightarrow \mathrm{GL}_{2}\left(\overline{\mathbf{F}}_{\ell}\right)$. Serre [Se2] defines the conductor $N(\bar{\rho})$ and weight $k(\bar{\rho})$ of $\bar{\rho}$. We call $\bar{\rho}$ modular if $\bar{\rho} \sim \bar{\rho}_{f, \lambda}$ for some eigenform $f$ and some place $\lambda \mid \ell$. We call $\bar{\rho}$ strongly modular if moreover we may take $f$ to have weight $k(\bar{\rho})$ and level $N(\bar{\rho})$. It is known from work of Mazur, Ribet, Carayol, Gross, Coleman, Voloch and others that for $\ell \geq 3$, $\bar{\rho}$ is strongly modular if and only if it is modular (see [Di1]). Serre has conjectured that all odd, irreducible $\bar{\rho}$ are strongly modular $\mathrm{Se} 2]$.

Now consider an elliptic curve $E_{/ \mathbf{Q}}$. Let $\rho_{E, \ell}\left(\operatorname{resp} . \bar{\rho}_{E, \ell}\right)$ denote the representation of $\operatorname{Gal}(\overline{\mathbf{Q}} / \mathbf{Q})$ on the $\ell$-adic Tate module (resp. the $\ell$-torsion) of $E(\overline{\mathbf{Q}})$. Let $N(E)$ denote the conductor of $E$. It is known that the following conditions are equivalent:

(1) The $L$-function $L(E, s)$ of $E$ equals the $L$-function $L(f, s)$ for some eigenform $f$.

(2) The $L$-function $L(E, s)$ of $E$ equals the $L$-function $L(f, s)$ for some eigenform $f$ of weight 2 and level $N(E)$.

(3) For some prime $\ell$, the representation $\rho_{E, \ell}$ is modular.

(4) For all primes $\ell$, the representation $\rho_{E, \ell}$ is modular.

(5) There is a non-constant holomorphic map $X_{1}(N)(\mathbf{C}) \rightarrow E(\mathbf{C})$ for some positive integer $N$.

(6) There is a non-constant morphism $X_{1}(N(E)) \rightarrow E$ which is defined over $\mathbf{Q}$.

The implications $(2) \Rightarrow(1),(4) \Rightarrow(3)$ and $(6) \Rightarrow(5)$ are tautological. The implication $(1) \Rightarrow(4)$ follows from the characterisation of $L(E, s)$ in terms of $\rho_{E, \ell}$. The implication $(3) \Rightarrow(2)$ follows from a theorem of Carayol Ca1]. The implication (2) $\Rightarrow(6)$ follows from a construction of Shimura [Sh2 and a theorem of Faltings [Fa]. The implication $(5) \Rightarrow(3)$ seems to have been first noticed by Mazur Maz. When these equivalent conditions are satisfied we call $E$ modular.

It has become a standard conjecture that all elliptic curves over $\mathbf{Q}$ are modular, although at the time this conjecture was first suggested the equivalence of the conditions above may not have been clear. Taniyama made a suggestion along the lines (1) as one of a series of problems collected at the Tokyo-Nikko conference in September 1955. However his formulation did not make clear whether $f$ should be a modular form or some more general automorphic form. He also suggested that constructions as in (5) and (6) might help attack this problem at least for some elliptic curves. In private conversations with a number of mathematicians (including Weil) in the early 1960's, Shimura suggested that assertions along the 
lines of (5) and (6) might be true (see [Sh3] and the commentary on [1967a] in We2 $)$. The first time such a suggestion appears in print is Weil's comment in We1 that assertions along the lines of (5) and (6) follow from the main result of that paper, a construction of Shimura and from certain "reasonable suppositions" and "natural assumptions". That assertion (1) is true for CM elliptic curves follows at once from work of Hecke and Deuring. Shimura Sh1 went on to check assertion (5) for these curves.

Our approach to Theorem $\mathrm{A}$ is an extension of the methods of Wiles Wi] and of Taylor and Wiles [TW]. We divide the proof into three cases.

(1) If $\left.\bar{\rho}_{E, 5}\right|_{\mathrm{Gal}(\overline{\mathbf{Q}} / \mathbf{Q}(\sqrt{5}))}$ is irreducible, we show that $\rho_{E, 5}$ is modular.

(2) If $\left.\bar{\rho}_{E, 5}\right|_{\mathrm{Gal}(\overline{\mathbf{Q}} / \mathbf{Q}(\sqrt{5}))}$ is reducible, but $\left.\bar{\rho}_{E, 3}\right|_{\mathrm{Gal}(\overline{\mathbf{Q}} / \mathbf{Q}(\sqrt{-3}))}$ is absolutely irreducible, we show that $\rho_{E, 3}$ is modular.

(3) If $\left.\bar{\rho}_{E, 5}\right|_{\mathrm{Gal}(\overline{\mathbf{Q}} / \mathbf{Q}(\sqrt{5}))}$ is reducible and $\left.\bar{\rho}_{E, 3}\right|_{\mathrm{Gal}(\overline{\mathbf{Q}} / \mathbf{Q}(\sqrt{-3}))}$ is absolutely reducible, then we show that $E$ is isogenous to an elliptic curve with $j$-invariant $0,(11 / 2)^{3}$, or $-5(29)^{3} / 2^{5}$ and so (from tables of modular elliptic curves of low conductor) is modular.

In each of cases $(1)$ and (2) there are two steps. First we prove that $\bar{\rho}_{E, \ell}$ is modular and then that $\rho_{E, \ell}$ is modular. In case (1) this first step is our Theorem $B$ and in case (2) it is a celebrated theorem of Langlands and Tunnell $[\mathrm{L},[\mathrm{T}]$. In fact, in both cases $E$ obtains semi-stable reduction over a tame extension of $\mathbf{Q}_{\ell}$ and the deduction of the modularity of $\rho_{E, \ell}$ from that of $\bar{\rho}_{E, \ell}$ was carried out in [CDT] by an extension of the methods of [Wi] and [TW]. In the third case we have to analyse the rational points on some modular curves of small level. This we did, with Elkies' help, in [CDT.

It thus only remained to prove Theorem $\mathrm{B}$. Let $\bar{\rho}$ be as in that theorem. Twisting by a quadratic character, we may assume that $\left.\bar{\rho}\right|_{\mathrm{Gal}\left(\overline{\mathbf{Q}}_{3} / \mathbf{Q}_{3}\right)}$ falls into one of the following cases (see 2.2 ):

(1) $\bar{\rho}$ is unramified at 3 .

(2) $\bar{\rho}\left(I_{3}\right)$ has order 5 .

(3) $\bar{\rho}\left(I_{3}\right)$ has order 4 .

(4) $\bar{\rho}\left(I_{3}\right)$ has order 12 and $\left.\bar{\rho}\right|_{\text {Gal }\left(\overline{\mathbf{Q}}_{3} / \mathbf{Q}_{3}\right)}$ has conductor 27.

(5) $\bar{\rho}\left(I_{3}\right)$ has order 3 .

(6) $\left.\bar{\rho}\right|_{\operatorname{Gal}\left(\overline{\mathbf{Q}}_{3} / \mathbf{Q}_{3}\right)}$ is induced from a character $\chi: \operatorname{Gal}\left(\overline{\mathbf{Q}}_{3} / \mathbf{Q}_{3}(\sqrt{-3})\right) \rightarrow \mathbf{F}_{25}^{\times}$such that $\chi(-1)=-1$ and

$$
\chi(\sqrt{-3})=\chi(1+3 \sqrt{-3})-\chi(1-3 \sqrt{-3}),
$$

where we use the Artin map (normalised to take uniformisers to arithmetic Frobenius) to identify $\chi$ with a character of $\mathbf{Q}_{3}(\sqrt{-3})^{\times}$.

We will refer to these as the $f=1,3,9,27,81$ and 243 cases respectively.

Using the technique of Minkowski and Klein (i.e. the observation that the moduli space of elliptic curves with full level 5 structure has genus 0 ; see for example [Kl]), Hilbert irreducibility and some local computations of Manoharmayum [Man], we find an elliptic curve $E_{/ \mathbf{Q}}$ with the following properties (see $\$ 2.2$ ):

- $\bar{\rho}_{E, 5} \sim \bar{\rho}$

- $\bar{\rho}_{E, 3}$ is surjective onto $\mathrm{GL}_{2}\left(\mathbf{F}_{3}\right)$, 
- and

(1) in the $f=1$ case, either $\left.\bar{\rho}_{E, 3}\right|_{I_{3}} \otimes \mathbf{F}_{9} \sim \omega_{2} \oplus \omega_{2}^{3}$ or

$$
\left.\bar{\rho}_{E, 3}\right|_{I_{3}} \sim\left(\begin{array}{cc}
\omega & * \\
0 & 1
\end{array}\right)
$$

and is peu ramifié;

(2) in the $f=3$ case,

$$
\left.\bar{\rho}_{E, 3}\right|_{I_{3}} \sim\left(\begin{array}{cc}
\omega & * \\
0 & 1
\end{array}\right)
$$

(3) in the $f=9$ case, $\left.\bar{\rho}_{E, 3}\right|_{I_{3}} \otimes \mathbf{F}_{9} \sim \omega_{2} \oplus \omega_{2}^{3}$;

(4) in the $f=27$ case,

$$
\left.\bar{\rho}_{E, 3}\right|_{I_{3}} \sim\left(\begin{array}{cc}
\omega & * \\
0 & 1
\end{array}\right)
$$

and is très ramifié;

(5) in the $f=81$ case,

$$
\left.\bar{\rho}_{E, 3}\right|_{I_{3}} \sim\left(\begin{array}{cc}
1 & * \\
0 & \omega
\end{array}\right)
$$

and is très ramifié;

(6) in the $f=243$ case,

$$
\left.\bar{\rho}_{E, 3}\right|_{\operatorname{Gal}\left(\overline{\mathbf{Q}}_{3} / \mathbf{Q}_{3}\right)} \sim\left(\begin{array}{cc}
\omega & * \\
0 & 1
\end{array}\right)
$$

is non-split over $\overline{\mathbf{Q}}_{3}^{\mathrm{ker} \bar{\rho}}$ and is très ramifié.

(We are using the terms très ramifié and peu ramifié in the sense of Serre [Se2]. We are also letting $\omega$ denote the $\bmod 3$ cyclotomic character and $\omega_{2}$ the second fundamental character $I_{3} \rightarrow \mathbf{F}_{9}^{\times}$, i.e.

$$
\omega_{2}(\sigma) \equiv \sigma(\sqrt[8]{3}) / \sqrt[8]{3} \bmod \sqrt[8]{3} .
$$

We will often use the equality $\omega=\omega^{-1}$ without further remark.) We emphasise that for a general elliptic curve over $\mathbf{Q}$ with $\bar{\rho}_{E, 5} \cong \bar{\rho}$, the representation $\bar{\rho}_{E, 3}$ does not have the above form, rather we are placing a significant restriction on $E$.

In each case our strategy is to prove that $\rho_{E, 3}$ is modular and so deduce that $\bar{\rho} \sim \bar{\rho}_{E, 5}$ is modular. Again we use the Langlands-Tunnell theorem to see that $\bar{\rho}_{E, 3}$ is modular and then an analogue of the arguments of [Wi and TW] to conclude that $\rho_{E, 3}$ is modular. This was carried out in Di2 in the cases $f=1$ and $f=3$, and in $[\mathrm{CDT}]$ in the case $f=9$. (In these cases the particular form of $\left.\bar{\rho}_{E, 3}\right|_{I_{3}}$ is not important.) This leaves the cases $f=27,81$ and 243, which are complicated by the fact that $E$ now only obtains good reduction over a wild extension of $\mathbf{Q}_{3}$. In these cases our argument relies essentially on the particular form we have obtained for $\left.\bar{\rho}_{E, 3}\right|_{\mathrm{Gal}\left(\overline{\mathbf{Q}}_{3} / \mathbf{Q}_{3}\right)}$ (depending on $\left.\bar{\rho}_{E, 5}\right|_{I_{3}}$ ). We do not believe that our methods for deducing the modularity of $\rho_{E, 3}$ from that of $\bar{\rho}_{E, 3}$ would work without this key simplification. It seems to be a piece of undeserved good fortune that for each possibility for $\left.\bar{\rho}\right|_{I_{3}}$ we can find a choice for $\left.\bar{\rho}_{E, 3}\right|_{\mathrm{Gal}\left(\overline{\mathbf{Q}}_{3} / \mathbf{Q}_{3}\right)}$ for which our methods work.

Following Wiles, to deduce the modularity of $\rho_{E, 3}$ from that of $\bar{\rho}_{E, 3}$, we consider certain universal deformations of $\bar{\rho}_{E, 3}$ and identify them with certain modular deformations which we realise over certain Hecke algebras. The key problem is to 
find the right local condition to impose on these deformations at the prime 3 . As in [CDT] we require that the deformations lie in the closure of the characteristic zero points which are potentially Barsotti-Tate (i.e. come from a 3-divisible group over the ring of integers of a finite extension of $\mathbf{Q}_{3}$ ) and for which the associated representation of the Weil group (see for example Appendix B of [CDT]) is of some specified form. That one can find suitable conditions on the representation of the Weil group at 3 for the arguments of [TW] to work seems to be a rare phenomenon in the wild case. It is here we make essential use of the fact that we need only treat our specific pairs $\left(\bar{\rho}_{E, 5}, \bar{\rho}_{E, 3}\right)$.

Our arguments follow closely the arguments of [CDT]. There are two main new features. Firstly, in the $f=243$ case, we are forced to specify the restriction of our representation of the Weil group completely, rather than simply its restriction to the inertia group as we have done in the past. Secondly, in the key computation of the local deformation rings, we now make use of a new description (due to Breuil) of finite flat group schemes over the ring of integers of any $p$-adic field in terms of certain (semi-)linear algebra data (see [Br2] and the summary [Br1]). This description enables us to make these computations. As the persistent reader will soon discover they are lengthy and delicate, particularly in the case $f=243$. It seems miraculous to us that these long computations with finite flat group schemes in $\$ 78$ and 9 give answers completely in accord with predictions made from much shorter computations with the local Langlands correspondence and the modular representation theory of $\mathrm{GL}_{2}\left(\mathbf{Q}_{3}\right)$ in $\$ 3$ We see no direct connection, but cannot help thinking that some such connection should exist.

Notation. In this paper $\ell$ denotes a rational prime. In $\$ 1.1, \$ 4.1$, $\$ 4.2$ and $\$ 4.3$ it is arbitrary. In the rest of $\$ 1$ and in $\$ 5$ we only assume it is odd. In the rest of the paper we only consider $\ell=3$.

If $F$ is a field we let $\bar{F}$ denote a separable closure, $F^{\text {ab }}$ the maximal subextension of $\bar{F}$ which is abelian over $F$ and $G_{F}$ the Galois group $\operatorname{Gal}(\bar{F} / F)$. If $F_{0}$ is a $p$-adic field (i.e. a finite extension of $\mathbf{Q}_{p}$ ) and $F^{\prime} / F_{0}$ a (possibly infinite) Galois extension, then we let $I_{F^{\prime}} / F_{0}$ denote the inertia subgroup of $\operatorname{Gal}\left(F^{\prime} / F_{0}\right)$. We also let $I_{F_{0}}$ denote $I_{\bar{F}_{0} / F_{0}}$, Frob $_{F_{0}} \in G_{F_{0}} / I_{F_{0}}$ denote the arithmetic Frobenius element and $W_{F_{0}}$ denote the Weil group of $F_{0}$, i.e. the dense subgroup of $G_{F_{0}}$ consisting of elements which map to an integer power of Frob $F_{0}$. We will normalise the Artin map of local class field theory so that uniformisers and arithmetic Frobenius elements correspond. (We apologise for this convention, which now seems to us a bad choice. However we feel it is important to stay consistent with [CDT].) We let $\mathcal{O}_{F_{0}}$ denote the ring of integers of $F_{0}, \wp_{F_{0}}$ the maximal ideal of $\mathcal{O}_{F_{0}}$ and $k_{F_{0}}$ the residue field $\mathcal{O}_{F_{0}} / \wp_{F_{0}}$. We write simply $G_{p}$ for $G_{\mathbf{Q}_{p}}, I_{p}$ for $I_{\mathbf{Q}_{p}}$ and Frob for Frob $\mathbf{Q}_{p}$. We also let $\mathbf{Q}_{p^{n}}$ denote the unique unramified degree $n$ extension of $\mathbf{Q}_{p}$ in $\overline{\mathbf{Q}}_{p}$. If $k$ is any perfect

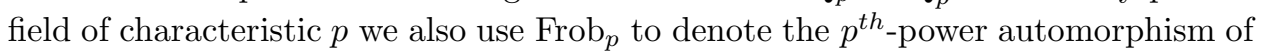
$k$ and its canonical lift to the Witt vectors $W(k)$.

We write $\epsilon$ for the $\ell$-adic cyclotomic character and sometimes $\omega$ for the reduction of $\epsilon$ modulo $\ell$. We write $\omega_{2}$ for the second fundamental character $I_{\ell} \rightarrow \mathbf{F}_{\ell^{2}}^{\times}$, i.e.

$$
\omega_{2}(\sigma) \equiv \sigma\left(\ell^{1 /\left(\ell^{2}-1\right)}\right) / \ell^{1 /\left(\ell^{2}-1\right)} \bmod \ell^{1 /\left(\ell^{2}-1\right)} .
$$

We also use $\omega$ and $\omega_{2}$ to denote the Teichmuller lifts of $\omega$ and $\omega_{2}$.

We let 1 denote the trivial character of a group. We will denote by $V^{\vee}$ the dual of a vector space $V$. 
If $g: A \rightarrow B$ is a homomorphism of rings and if $X / \operatorname{Spec} A$ is an $A$-scheme, then we sometimes write ${ }^{g} X$ for the pullback of $X$ by Spec $g$. We adopt this notation so that ${ }^{g}\left({ }^{h} X\right)={ }^{g h} X$. Similarly if $\theta: X \rightarrow Y$ is a morphism of schemes over $A$ we will sometimes write ${ }^{g} \theta$ for the pullback of $\theta$ by $\operatorname{Spec} g$.

By finite flat group scheme we always mean commutative finite flat group scheme. If $F_{0}$ is a field of characteristic 0 with fixed algebraic closure $\bar{F}_{0}$ we use without comment the canonical identification of finite flat $F_{0}$-group schemes with finite discrete $\operatorname{Gal}\left(\bar{F}_{0} / F_{0}\right)$-modules, and we will say that such objects correspond. If $R$ is a Dedekind domain with field of fractions $F$ of characteristic 0 , then by a model of a finite flat $F$-group scheme $G$ we mean a finite locally free $R$-group scheme $\mathcal{G}$ and an isomorphism $i: G \stackrel{\sim}{\rightarrow} \mathcal{G} \times F^{\prime}$. As in Proposition 2.2.2 of $\mathrm{Ra}$ ] the isomorphism classes of models for $G$ form a lattice $\left((\mathcal{G}, i) \geq\left(\mathcal{G}^{\prime}, i^{\prime}\right)\right.$ if there exists a map of finite flat group schemes $\mathcal{G} \rightarrow \mathcal{G}^{\prime}$ compatible with $i$ and $\left.i^{\prime}\right)$ and we can talk about the inf and sup of two such models. If $R$ is also local we call the model $(\mathcal{G}, i)$ local-local if its special fibre is local-local. When the ring $R$ is understood we sometimes simply refer to $(\mathcal{G}, i)$, or even just $\mathcal{G}$, as an integral model of $G$.

We use Serre's terminology peu ramifié and très ramifié; see [Se2].

\section{Contents}

Introduction 843

Notation $\quad 848$

1. Types 850

1.1. Types of local deformations $\quad 850$

1.2. Types for admissible representations $\quad 852$

1.3. Reduction of types for admissible representations

1.4. The main theorems 857

2. Examples and applications $\quad 860$

2.1. Examples 860

2.2. Applications 862

2.3. An extension of a result of Manoharmayum 865

3. Admittance 866

3.1. The case of $\tau_{1} \quad 866$

3.2. The case of $\tau_{-1} \quad 866$

\begin{tabular}{lll} 
3.3. The case of $\tau_{ \pm 3}$ & 867 \\
\hline
\end{tabular}

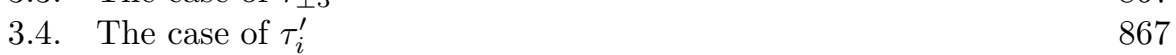

4. New deformation problems 867

4.1. Some generalities on group schemes $\quad 868$

4.2. Filtrations 870

4.3. Generalities on deformation theory 874

4.4. Reduction steps for Theorem 2.1.2 875

4.5. Reduction steps for Theorem 2.1.4 876

\begin{tabular}{ll|l|} 
4.6. Reduction steps for Theorem 2.1.6 & 877 \\
\hline
\end{tabular}

4.7. Some Galois cohomology $\quad 880$

5. Breuil modules 883

5.1. Basic properties of Breuil modules $\quad 883$

\begin{tabular}{ll|}
\hline 5.2 . Examples & 886 \\
\hline 5.3.
\end{tabular}

\begin{tabular}{ll|l|}
\hline 5.3. Relationship to syntomic sheaves & 888 \\
\hline
\end{tabular}

5.4. Base change 890 
5.5. Reformulation 894

5.6. Descent data 896

5.7. More examples 898

6. Some local fields $\quad 899$

6.1. The case of $F_{1}^{\prime} \quad 899$

6.2. The case of $F_{-1}^{\prime} \quad 900$

6.3. The case of $F_{3}^{\prime} \quad 900$

6.4. The case of $F_{-3}^{\prime} \quad 901$

6.5. The case of $F_{i}^{\prime} \quad 1902$

7. Proof of Theorem 4.4.1 904

7.1. Rank one calculations $\quad 904$

7.2. Rank two calculations $\quad 904$

7.3. Rank three calculations $\quad 908$

7.4. Conclusion of the proof of Theorem 4.4.1 909

8. Proof of Theorem 4.5.1 911

8.1. Rank one calculations 911

8.2. Models for $\bar{\rho} \quad 912$

8.3. Completion of the proof of Theorem 4.5.1 917

9. Proof of Theorems 4.6.1, 4.6.2 and 4.6.3 919

9.1. Rank one calculations $\quad 919$

9.2. Models for $\bar{\rho} \quad 920$

9.3. Further rank two calculations 927

9.4. Completion of the proof of Theorem 4.6.1 931

9.5. Completion of the proof of Theorem 4.6.3 932

9.6. Completion of the proof of Theorem 4.6.2

10. Corrigenda for [CDT]

Acknowledgements $\quad 938$

References $\quad 938$

\section{TYPES}

1.1. Types of local deformations. By an $\ell$-type we mean an equivalence class of two-dimensional representations

$$
\tau: I_{\ell} \rightarrow \mathrm{GL}(D)
$$

over $\overline{\mathbf{Q}}_{\ell}$ which have open kernel and which can be extended to a representation of $W_{\mathbf{Q}_{\ell}}$. By an extended $\ell$-type we shall simply mean an equivalence class of twodimensional representations

$$
\tau^{\prime}: W_{\mathbf{Q}_{\ell}} \rightarrow \mathrm{GL}\left(D^{\prime}\right)
$$

over $\overline{\mathbf{Q}}_{\ell}$ with open kernel.

Suppose that $\tau$ is an $\ell$-type and that $K$ is a finite extension of $\mathbf{Q}_{\ell}$ in $\overline{\mathbf{Q}}_{\ell}$. Recall from [CDT] that a continuous representation $\rho$ of $G_{\ell}$ on a two-dimensional $K$-vector space $M$ is said to be of type $\tau$ if

(1) $\rho$ is Barsotti-Tate over $F$ for any finite extension $F$ of $\mathbf{Q}_{\ell}$ such that $\left.\tau\right|_{I_{F}}$ is trivial;

(2) the restriction of $W D(\rho)$ to $I_{\ell}$ is in $\tau$;

(3) the character $\epsilon^{-1} \operatorname{det} \rho$ has finite order prime to $\ell$. 
(For the definition of "Barsotti-Tate" and of the representation $W D(\rho)$ associated to a potentially Barsotti-Tate representation, see $\S 1.1$ and Appendix B of [CDT].) Similarly if $\tau^{\prime}$ is an extended $\ell$-type, then we say that $\rho$ is of extended type $\tau^{\prime}$ if

(1) $\rho$ is Barsotti-Tate over $F$ for any finite extension $F$ of $\mathbf{Q}_{\ell}$ such that $\left.\tau^{\prime}\right|_{I_{F}}$ is trivial;

(2) $W D(\rho)$ is equivalent to $\tau^{\prime}$;

(3) the character $\epsilon^{-1} \operatorname{det} \rho$ has finite order prime to $\ell$.

Note that no representation can have extended type $\tau^{\prime}$ unless $\operatorname{det} \tau^{\prime}$ is of the form $\chi_{1} \chi_{2}$ where $\chi_{1}$ has finite order prime to $\ell$ and where $\chi_{2}$ is unramified and takes an arithmetic Frobenius element to $\ell$; see Appendix B of [CDT]. (Using Theorem 1.4 of [Br2], one can show that for $\ell$ odd one obtains equivalent definitions of "type $\tau$ " and "extended type $\tau^{\prime \prime}$ " if one weakens the first assumption to simply require that $\rho$ is potentially Barsotti-Tate.)

Now fix a finite extension $K$ of $\mathbf{Q}_{\ell}$ in $\overline{\mathbf{Q}}_{\ell}$ over which $\tau$ (resp. $\tau^{\prime}$ ) is rational. Let $\mathcal{O}$ denote the integers of $K$ and let $k$ denote the residue field of $\mathcal{O}$. Let

$$
\bar{\rho}: G_{\ell} \longrightarrow \mathrm{GL}(V)
$$

be a continuous representation of $G_{\ell}$ on a two-dimensional $k$-vector space $V$ and suppose that $\operatorname{End}_{k\left[G_{\ell}\right]} V=k$. One then has a universal deformation ring $R_{V, \mathcal{O}}$ for $\bar{\rho}$ (see, for instance, Appendix A of [CDT]).

We say that a prime ideal $\mathfrak{p}$ of $R_{V, \mathcal{O}}$ is of type $\tau$ (resp. of extended type $\tau^{\prime}$ ) if there exist a finite extension $K^{\prime}$ of $K$ in $\overline{\mathbf{Q}}_{\ell}$ and an $\mathcal{O}$-algebra homomorphism $R_{V, \mathcal{O}} \rightarrow K^{\prime}$ with kernel $\mathfrak{p}$ such that the pushforward of the universal deformation of $\rho$ over $R_{V, \mathcal{O}}$ to $K^{\prime}$ is of type $\tau$ (resp. of extended type $\tau^{\prime}$ ).

Let $\tau$ be an $\ell$-type and $\tau^{\prime}$ an irreducible extended $\ell$-type. If there do not exist any prime ideals $\mathfrak{p}$ of type $\tau$ (resp. of extended type $\tau^{\prime}$ ), we define $R_{V, \mathcal{O}}^{D}=0$ (resp. $\left.R_{V, \mathcal{O}}^{D^{\prime}}=0\right)$. Otherwise, define $R_{V, \mathcal{O}}^{D}$ (resp. $\left.R_{V, \mathcal{O}}^{D^{\prime}}\right)$ to be the quotient of $R_{V, \mathcal{O}}$ by the intersection of all $\mathfrak{p}$ of type $\tau$ (resp. of extended type $\tau^{\prime}$ ). We will sometimes write $R_{V, \mathcal{O}}^{\tau}\left(\operatorname{resp} . R_{V, \mathcal{O}}^{\tau^{\prime}}\right)$ for $R_{V, \mathcal{O}}^{D}\left(\operatorname{resp} . R_{V, \mathcal{O}}^{D^{\prime}}\right)$. We say that a deformation of $\bar{\rho}$ is weakly of type $\tau$ (resp. weakly of extended type $\tau^{\prime}$ ) if the associated local $\mathcal{O}$-algebra map $R_{V, \mathcal{O}} \rightarrow R$ factors through the quotient $R_{V, \mathcal{O}}^{D}$ (resp. $R_{V, \mathcal{O}}^{D^{\prime}}$ ). We say that $\tau$ (resp. $\tau^{\prime}$ ) is weakly acceptable for $\bar{\rho}$ if either $R_{V, \mathcal{O}}^{D}=0$ (resp. $R_{V, \mathcal{O}}^{D^{\prime}}=0$ ) or there is a surjective local $\mathcal{O}$-algebra map $\mathcal{O} \llbracket X \rrbracket \rightarrow R_{V, \mathcal{O}}^{D}\left(\right.$ resp. $\mathcal{O} \llbracket X \rrbracket \rightarrow R_{V, \mathcal{O}}^{D^{\prime}}$ ). We say that $\tau$ (resp. $\tau^{\prime}$ ) is acceptable for $\bar{\rho}$ if $R_{V, \mathcal{O}}^{D} \neq 0$ (resp. $R_{V, \mathcal{O}}^{D^{\prime}} \neq 0$ ) and if there is a surjective local $\mathcal{O}$-algebra map $\mathcal{O} \llbracket X \rrbracket \rightarrow R_{V, \mathcal{O}}^{D}\left(\right.$ resp. $\left.\mathcal{O} \llbracket X \rrbracket \rightarrow R_{V, \mathcal{O}}^{D^{\prime}}\right)$.

If $K^{\prime}$ is a finite extension of $K$ in $\overline{\mathbf{Q}}_{\ell}$ with valuation ring $\mathcal{O}^{\prime}$ and residue field $k^{\prime}$, then $\mathcal{O}^{\prime} \otimes_{\mathcal{O}} R_{V, \mathcal{O}}^{D}$ (resp. $\mathcal{O}^{\prime} \otimes_{\mathcal{O}} R_{V, \mathcal{O}}^{D^{\prime}}$ ) is naturally isomorphic to $R_{V \otimes_{k} k^{\prime}, \mathcal{O}^{\prime}}^{D}$ (resp. $R_{V \otimes_{k} k^{\prime}, \mathcal{O}^{\prime}}^{D^{\prime}}$ ). Thus (weak) acceptability depends only on $\tau$ (resp. $\tau^{\prime}$ ) and $\bar{\rho}$, and not on the choice of $K$. Moreover $\tau$ (resp. $\tau^{\prime}$ ) is acceptable for $\bar{\rho}$ if and only if $\tau$ (resp. $\left.\tau^{\prime}\right)$ is acceptable for $\bar{\rho} \otimes_{k} k^{\prime}$.

Although it is of no importance for the sequel, we make the following conjecture, part of which we already conjectured as Conjecture 1.2.1 of [CDT].

Conjecture 1.1.1. Suppose that $\tau$ is an $\ell$-type and $\tau^{\prime}$ an absolutely irreducible extended $\ell$-type. A deformation $\rho: G_{\ell} \rightarrow \mathrm{GL}(M)$ of $\bar{\rho}$ to the ring of integers $\mathcal{O}^{\prime}$ of a finite extension $K^{\prime} / K$ in $\overline{\mathbf{Q}}_{\ell}$ is weakly of type $\tau$ (resp. weakly of extended $\ell$-type $\left.\tau^{\prime}\right)$ if and only if $M$ is of type $\tau$ (resp. of extended type $\tau^{\prime}$ ). 
If $\tau$ is a tamely ramified $\ell$-type, then we expect that it is frequently the case that $\tau$ is acceptable for residual representations $\bar{\rho}$, as in Conjectures 1.2.2 and 1.2.3 of [CDT]. On the other hand if $\tau$ (resp. $\tau^{\prime}$ ) is a wildly ramified $\ell$-type (resp. wildly ramified extended $\ell$-type), then we expect that it is rather rare that $\tau$ (resp. $\tau^{\prime}$ ) is acceptable for a residual representation $\bar{\rho}$. In this paper we will be concerned with a few wild cases for the prime $\ell=3$ which do turn out to be acceptable.

1.2. Types for admissible representations. From now on we assume that $\ell$ is odd. If $F$ is a finite extension of $\mathbf{Q}_{\ell}$ we will identify $F^{\times}$with $W_{F}^{\text {ab }}$ via the Artin map. Let $U_{0}\left(\ell^{r}\right)$ denote the subgroup of $\mathrm{GL}_{2}\left(\mathbf{Z}_{\ell}\right)$ consisting of elements with upper triangular $\bmod \ell^{r}$ reduction. Also let $\widetilde{U}_{0}(\ell)$ denote the normaliser of $U_{0}(\ell)$ in $\mathrm{GL}_{2}\left(\mathbf{Q}_{\ell}\right)$. Thus $\widetilde{U}_{0}(\ell)$ is generated by $U_{0}(\ell)$ and by

$$
w_{\ell}=\left(\begin{array}{cc}
0 & -1 \\
\ell & 0
\end{array}\right) \text {. }
$$

If $\tau$ is an $\ell$-type, set $U_{\tau}=\mathrm{GL}_{2}\left(\mathbf{Z}_{\ell}\right)$ if $\tau$ is reducible and $U_{\tau}=U_{0}(\ell)$ if $\tau$ is irreducible. If $\tau^{\prime}$ is an extended $\ell$-type with $\left.\tau^{\prime}\right|_{I_{\ell}}$ irreducible, set $U_{\tau^{\prime}}=\widetilde{U}_{0}(\ell)$. In this subsection we will associate to an $\ell$-type $\tau$ an irreducible representation $\sigma_{\tau}$ of $U_{\tau}$ over $\overline{\mathbf{Q}}_{\ell}$ with open kernel, and to an extended $\ell$-type $\tau^{\prime}$ with $\left.\tau^{\prime}\right|_{I_{\ell}}$ irreducible an irreducible representation $\sigma_{\tau^{\prime}}$ of $U_{\tau^{\prime}}$ over $\overline{\mathbf{Q}}_{\ell}$ with open kernel. We need to consider several cases, which we treat one at a time.

First suppose that $\tau=\left.\left.\chi_{1}\right|_{I_{\ell}} \oplus \chi_{2}\right|_{I_{\ell}}$ where each $\chi_{i}$ is a character of $W_{\mathbf{Q}_{\ell}}$. Let $a$ denote the conductor of $\chi_{1} / \chi_{2}$. If $a=0$, then set

$$
\sigma_{\tau}=\operatorname{St} \otimes\left(\chi_{1} \circ \text { det }\right)=\operatorname{St} \otimes\left(\chi_{2} \circ \text { det }\right),
$$

where St denotes the Steinberg representation of $\mathrm{PGL}_{2}\left(\mathbf{F}_{\ell}\right)$. Now suppose that $a>0$. Let $\sigma_{\tau}$ denote the induction from $U_{0}\left(\ell^{a}\right)$ to $\mathrm{GL}_{2}\left(\mathbf{Z}_{\ell}\right)$ of the character of $U_{0}\left(\ell^{a}\right)$ which sends

$$
\left(\begin{array}{cc}
\alpha & \beta \\
\ell^{a} \gamma & \delta
\end{array}\right) \longmapsto\left(\chi_{1} / \chi_{2}\right)(\alpha) \chi_{2}\left(\alpha \delta-\ell^{a} \beta \gamma\right)
$$

This is irreducible and does not depend on the ordering of $\chi_{1}$ and $\chi_{2}$.

For the next case, let $F$ denote the unramified quadratic extension of $\mathbf{Q}_{\ell}$ and $s$ the non-trivial automorphism of $F$ over $\mathbf{Q}_{\ell}$. Suppose that $\tau$ is the restriction to $I_{\ell}$ of the induction from $W_{F}$ to $W_{\mathbf{Q}_{\ell}}$ of a character $\chi$ of $W_{F}$ with $\chi \neq \chi^{s}$. Let $a$ denote the conductor of $\chi / \chi^{s}$, so that $a>0$. Choose a character $\chi^{\prime}$ of $W_{\mathbf{Q}_{\ell}}$ such that $\left.\chi^{\prime}\right|_{W_{F}} ^{-1} \chi$ has conductor $a$. If $a=1$ we set

$$
\sigma_{\tau}=\Theta\left(\left.\chi^{\prime}\right|_{W_{F}} ^{-1} \chi\right) \otimes\left(\chi^{\prime} \circ \operatorname{det}\right),
$$

where $\Theta(\cdot)$ is the irreducible representation of $\mathrm{GL}_{2}\left(\mathbf{F}_{\ell}\right)$ defined on page 532 of CDT.

To define $\sigma_{\tau}$ for $a>1$ we will identify $\mathrm{GL}_{2}\left(\mathbf{Z}_{\ell}\right)$ with the automorphisms of the $\mathbf{Z}_{\ell^{-}}$ module $\mathcal{O}_{F}$. If $a$ is even, then we let $\sigma_{\tau}$ denote the induction from $\mathcal{O}_{F}^{\times}\left(1+\ell^{a / 2} \mathcal{O}_{F} s\right)$ to $\mathrm{GL}_{2}\left(\mathbf{Z}_{\ell}\right)$ of the character $\varphi$ of $\mathcal{O}_{F}^{\times}\left(1+\ell^{a / 2} \mathcal{O}_{F} s\right)$, where, for $\alpha \in \mathcal{O}_{F}^{\times}$and $\beta \in$ $\left(1+\ell^{a / 2} \mathcal{O}_{F} s\right)$,

$$
\varphi(\alpha \beta)=\left(\left.\chi^{\prime}\right|_{W_{F}} ^{-1} \chi\right)(\alpha) \chi^{\prime}(\operatorname{det} \alpha \beta) .
$$

If $a>1$ is odd, then we let $\sigma_{\tau}$ denote the induction from $\mathcal{O}_{F}^{\times}\left(1+\ell^{(a-1) / 2} \mathcal{O}_{F} s\right)$ to $\mathrm{GL}_{2}\left(\mathbf{Z}_{\ell}\right)$ of $\eta$, where $\eta$ is the $\ell$-dimensional irreducible representation of 
$\mathcal{O}_{F}^{\times}\left(1+\ell^{(a-1) / 2} \mathcal{O}_{F} s\right)$ such that $\left.\eta\right|_{\mathcal{O}_{F}^{\times}\left(1+\ell^{(a+1) / 2} \mathcal{O}_{F} s\right)}$ is the direct sum of the characters

$$
\alpha \beta \longmapsto\left(\left.\chi^{\prime}\right|_{W_{F}} ^{-1} \chi \chi^{\prime \prime}\right)(\alpha) \chi^{\prime}(\operatorname{det} \alpha \beta)
$$

for $\alpha \in \mathcal{O}_{F}^{\times}$and $\beta \in\left(1+\ell^{(a+1) / 2} \mathcal{O}_{F} s\right)$, where $\chi^{\prime \prime}$ runs over the $\ell$ non-trivial characters of $\mathcal{O}_{F}^{\times} / \mathbf{Z}_{\ell}^{\times}\left(1+\ell \mathcal{O}_{F}\right)$.

Now suppose $\tau^{\prime}$ is an extended type such that $\left.\tau^{\prime}\right|_{I_{\ell}}$ is irreducible. There is a ramified quadratic extension $F / \mathbf{Q}_{\ell}$ and a character $\chi$ of $W_{F}$ such that the induction from $W_{F}$ to $W_{\mathbf{Q}_{\ell}}$ of $\chi$ is $\tau^{\prime}$ (see $\S 2.6$ of [G]). Let $s$ denote the non-trivial field automorphism of $F$ over $\mathbf{Q}_{\ell}$ and also let $\wp_{F}$ denote the maximal ideal of the ring of integers $\mathcal{O}_{F}$ of $F$. Let $a$ denote the conductor of $\chi / \chi^{s}$, so $a$ is even and $a \geq 2$. We may choose a character $\chi^{\prime}$ of $W_{\mathbf{Q}_{\ell}}$ such that $\left.\chi^{\prime}\right|_{W_{F}} ^{-1} \chi$ has conductor $a$. We will identify $\mathrm{GL}_{2}\left(\mathbf{Q}_{\ell}\right)$ with the automorphisms of the $\mathbf{Q}_{\ell}$ vector space $F$. We will also identify $U_{0}(\ell)$ with the stabiliser of the pair of lattices $\wp_{F}^{-1} \supset \mathcal{O}_{F}$. We define $\sigma_{\tau^{\prime}}$ to be the induction from $F^{\times}\left(1+\wp_{F}^{a / 2} s\right)$ to $\widetilde{U}_{0}(\ell)$ of the character $\varphi$ of $F^{\times}\left(1+\wp_{F}^{a / 2} s\right)$, where

$$
\varphi(\alpha \beta)=\left(\left.\chi^{\prime}\right|_{W_{F}} ^{-1} \chi \chi^{\prime \prime}\right)(\alpha) \chi^{\prime}(\operatorname{det} \alpha \beta),
$$

with $\alpha \in F^{\times}$and $\beta \in\left(1+\wp_{F}^{a / 2} s\right)$, where $\chi^{\prime \prime}$ is a character of $F^{\times} /\left(\mathcal{O}_{F}^{\times}\right)^{2}$ defined as follows. Let $\psi$ be a character of $\mathbf{Q}_{\ell}$ with kernel $\mathbf{Z}_{\ell}$. Choose $\theta \in F^{\times}$such that for $x \in \wp_{F}^{a-1}$ we have

$$
\left(\left.\chi^{\prime}\right|_{W_{F}} ^{-1} \chi\right)(1+x)=\psi\left(\operatorname{tr}_{F / \mathbf{Q}_{\ell}}(\theta x)\right) .
$$

We impose the following conditions which determine $\chi^{\prime \prime}$ :

- $\chi^{\prime \prime}$ is a character of $F^{\times} /\left(\mathcal{O}_{F}^{\times}\right)^{2}$;

- $\left.\chi^{\prime \prime}\right|_{\mathcal{O}_{F}^{\times}}$is non-trivial;

- and

$$
\chi^{\prime \prime}\left(-\theta\left(N_{F / \mathbf{Q}_{\ell}} \varpi\right)^{a / 2}\right)=\sum_{x \in \mathbf{Z} / \ell \mathbf{Z}} \psi\left(x^{2} / N_{F / \mathbf{Q}_{\ell}} \varpi\right),
$$

where $\varpi$ is a uniformiser in $\mathcal{O}_{F}$.

Finally if $\tau$ is an irreducible $\ell$-type, choose an extended $\ell$-type $\tau^{\prime}$ which restricts to $\tau$ on $I_{\ell}$ and set $\sigma_{\tau}=\left.\sigma_{\tau^{\prime}}\right|_{U_{0}(\ell)}$.

We remark that these definitions are independent of any choices (see [G]).

Recall that by the local Langlands conjecture we can associate to an irreducible admissible representation $\pi$ of $\mathrm{GL}_{2}\left(\mathbf{Q}_{\ell}\right)$ a two-dimensional representation $W D(\pi)$ of $W_{\mathbf{Q}_{\ell}}$. (See $\S 4.1$ of $[\overline{C D T}]$ for the normalisation we use.)

Lemma 1.2.1. Suppose that $\tau$ is an $\ell$-type and that $\tau^{\prime}$ is an extended $\ell$-type with $\left.\tau^{\prime}\right|_{I_{\ell}}$ irreducible. Suppose also that $\pi$ is an infinite-dimensional irreducible admissible representation of $\mathrm{GL}_{2}\left(\mathbf{Q}_{\ell}\right)$ over $\overline{\mathbf{Q}}_{\ell}$. Then:

(1) $\sigma_{\tau}$ and $\sigma_{\tau^{\prime}}$ are irreducible.

(2) If $\left.W D(\pi)\right|_{I_{\ell}} \sim \tau\left(\right.$ resp. $\left.W D(\pi) \sim \tau^{\prime}\right)$, then

$$
\operatorname{Hom}_{U_{\tau}}\left(\sigma_{\tau}, \pi\right) \cong \overline{\mathbf{Q}}_{\ell}
$$

(resp.

$$
\left.\operatorname{Hom}_{U_{\tau^{\prime}}}\left(\sigma_{\tau^{\prime}}, \pi\right) \cong \overline{\mathbf{Q}}_{\ell}\right)
$$


(3) If $\left.W D(\pi)\right|_{I_{\ell}} \neq \tau\left(\right.$ resp. $\left.W D(\pi) \neq \tau^{\prime}\right)$, then

$$
\operatorname{Hom}_{U_{\tau}}\left(\sigma_{\tau}, \pi\right)=(0)
$$

(resp.

$$
\left.\operatorname{Hom}_{\tau_{\tau^{\prime}}}\left(\sigma_{\tau^{\prime}}, \pi\right)=(0)\right) \text {. }
$$

Proof. The case that $\tau$ extends to a reducible representation of $W_{\mathbf{Q}_{\ell}}$ follows from the standard theory of principal series representations for $\mathrm{GL}_{2}\left(\mathbf{Q}_{\ell}\right)$. The case that $\tau$ is reducible but does not extend to a reducible representation of $W_{\mathbf{Q}_{\ell}}$ follows from Theorem 3.7 of [G]. The case of $\tau^{\prime}$ follows from Theorem 4.6 of [G].

Thus, suppose that $\tau$ is an irreducible $\ell$-type and that $\tau^{\prime}$ is an extension of $\tau$ to an extended $\ell$-type. If $\delta$ denotes the unramified quadratic character of $W_{\mathbf{Q}_{\ell}}$, then $\tau^{\prime} \nsim \tau^{\prime} \otimes \delta$ and so we deduce that

$$
\sigma_{\tau^{\prime}} \not \sigma_{\tau^{\prime} \otimes \delta} \sim \sigma_{\tau^{\prime}} \otimes(\delta \circ \text { det }) .
$$

Thus $\left.\sigma_{\tau^{\prime}}\right|_{\mathbf{Q}_{\ell}^{\times} U_{0}(\ell)}$ is irreducible. It follows that $\sigma_{\tau}$ is irreducible. The second and third part of the lemma for $\tau$ follow similarly.

1.3. Reduction of types for admissible representations. We begin by reviewing some irreducible representations of $\mathrm{GL}_{2}\left(\mathbf{Z}_{\ell}\right), U_{0}(\ell)$ and $\widetilde{U}_{0}(\ell)$. Let $\sigma_{1,0}$ denote the standard representation of $\mathrm{GL}_{2}\left(\mathbf{F}_{\ell}\right)$ over $\overline{\mathbf{F}}_{\ell}$. If $n=0,1, \ldots, \ell-1$ and if $m \in \mathbf{Z} /(\ell-1) \mathbf{Z}$, then we let $\sigma_{n, m}=\operatorname{Symm}^{n}\left(\sigma_{1,0}\right) \otimes \operatorname{det}^{m}$. We may think of $\sigma_{n, m}$ as a continuous representation of $\mathrm{GL}_{2}\left(\mathbf{Z}_{\ell}\right)$ over $\overline{\mathbf{F}}_{\ell}$. These representations are irreducible, mutually non-isomorphic and exhaust the irreducible continuous representations of $\mathrm{GL}_{2}\left(\mathbf{Z}_{\ell}\right)$ over $\overline{\mathbf{F}}_{\ell}$.

If $m_{1}, m_{2} \in \mathbf{Z} /(\ell-1) \mathbf{Z}$ we let $\sigma_{m_{1}, m_{2}}^{\prime}$ denote the character of $U_{0}(\ell)$ over $\overline{\mathbf{F}}_{\ell}$ determined by

$$
\left(\begin{array}{cc}
a & b \\
\ell c & d
\end{array}\right) \longmapsto a^{m_{1}} d^{m_{2}}
$$

These representations are irreducible, mutually non-isomorphic, and exhaust the irreducible continuous representations of $U_{0}(\ell)$ over $\overline{\mathbf{F}}_{\ell}$.

If $m_{1}, m_{2} \in \mathbf{Z} /(\ell-1) \mathbf{Z}, a \in \overline{\mathbf{F}}_{\ell}^{\times}$and $m_{1} \neq m_{2}$, then we let $\sigma_{\left\{m_{1}, m_{2}\right\}, a}^{\prime}$ denote the representation of $\widetilde{U}_{0}(\ell)$ over $\overline{\mathbf{F}}_{\ell}$ obtained by inducing the character of $\mathbf{Q}_{\ell}^{\times} U_{0}(\ell)$ which restricts to $\sigma_{m_{1}, m_{2}}^{\prime}$ on $U_{0}(\ell)$ and which sends $-\ell$ to $a$. If $m \in \mathbf{Z} /(\ell-1) \mathbf{Z}$ and $a \in \overline{\mathbf{F}}_{\ell}^{\times}$, then we let $\sigma_{\{m\}, a}^{\prime}$ denote the character of $\widetilde{U}_{0}(\ell)$ over $\overline{\mathbf{F}}_{\ell}$ which restricts to $\sigma_{m, m}^{\prime}$ on $U_{0}(\ell)$ and which sends $w_{\ell}$ to $a$. These representations are irreducible, mutually non-isomorphic and exhaust the irreducible, finite-dimensional, continuous representations of $\widetilde{U}_{0}(\ell)$ over $\overline{\mathbf{F}}_{\ell}$.

We will say that a reducible $\ell$-type $\tau$ (resp. irreducible $\ell$-type, resp. extended $\ell$-type $\tau$ with irreducible restriction to $I_{\ell}$ ) admits an irreducible representation $\sigma$ of $\mathrm{GL}_{2}\left(\mathbf{Z}_{\ell}\right)$ (resp. $U_{0}(\ell)$, resp. $\widetilde{U}_{0}(\ell)$ ) over $\overline{\mathbf{F}}_{\ell}$, if $\sigma_{\tau}$ (resp. $\sigma_{\tau}$, resp. $\sigma_{\tau^{\prime}}$ ) contains an invariant $\mathcal{O}_{\overline{\mathbf{Q}}_{\ell}}$-lattice $\Lambda$ and if $\sigma$ is a Jordan-Hölder constituent of $\Lambda \otimes \overline{\mathbf{F}}_{\ell}$. We will say that $\tau$ (resp. $\tau$, resp. $\tau^{\prime}$ ) simply admits $\sigma$ if $\sigma$ is a Jordan-Hölder constituent of $\Lambda \otimes \overline{\mathbf{F}}_{\ell}$ of multiplicity one.

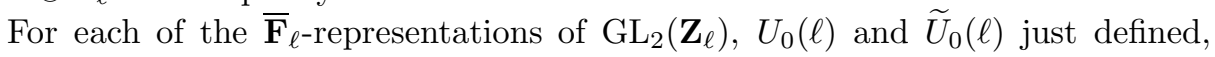
we wish to define notions of "admittance" and "simple admittance" with respect to a continuous representation $\bar{\rho}: G_{\ell} \rightarrow \mathrm{GL}_{2}\left(\overline{\mathbf{F}}_{\ell}\right)$. Let $\bar{\rho}$ be a fixed continuous representation $G_{\ell} \rightarrow \mathrm{GL}_{2}\left(\overline{\mathbf{F}}_{\ell}\right)$. 
- The representation $\sigma_{n, m}$ admits $\bar{\rho}$ if either

$$
\left.\bar{\rho}\right|_{I_{\ell}} \sim\left(\begin{array}{cc}
\omega_{2}^{1-\ell n-m(\ell+1)} & 0 \\
0 & \omega_{2}^{\ell-n-m(\ell+1)}
\end{array}\right)
$$

or

$$
\left.\bar{\rho}\right|_{I_{\ell}} \sim\left(\begin{array}{cc}
\omega^{1-m} & * \\
0 & \omega^{-n-m}
\end{array}\right),
$$

which in addition we require to be peu-ramifié in the case $n=0$. (Note that $\sigma_{n, 0}$ admits $\bar{\rho}$ if and only if the Serre weight (see $[$ Se2 $)$ of $\bar{\rho}^{\vee} \otimes \omega$ is $n+2$.)

- The representation $\sigma_{n, m}$ simply admits $\bar{\rho}$ if $\sigma_{n, m}$ admits $\bar{\rho}$.

- The representation $\sigma_{m_{1}, m_{2}}^{\prime}$ admits $\bar{\rho}$ if either

$$
\left.\bar{\rho}\right|_{I_{\ell}} \sim\left(\begin{array}{cc}
\omega_{2}^{1-\ell m_{i}-m_{j}} & 0 \\
0 & \omega_{2}^{\ell-m_{i}-\ell m_{j}}
\end{array}\right),
$$

where $\left\{m_{i}, m_{j}\right\}=\left\{m_{1}, m_{2}\right\}$ and $m_{i} \geq m_{j}$, or

$$
\left.\bar{\rho}\right|_{I_{\ell}} \sim\left(\begin{array}{cc}
\omega^{1-m_{1}} & * \\
0 & \omega^{-m_{2}}
\end{array}\right) \text {, }
$$

or

$$
\left.\bar{\rho}\right|_{I_{\ell}} \sim\left(\begin{array}{cc}
\omega^{1-m_{2}} & * \\
0 & \omega^{-m_{1}}
\end{array}\right) .
$$

(Note that $\sigma_{m_{1}, m_{2}}^{\prime}$ admits $\bar{\rho}$ if and only if some irreducible constituent of $\operatorname{Ind}_{U_{0}(\ell)}^{\mathrm{GL}_{2}\left(\mathbf{Z}_{\ell}\right)} \sigma_{m_{1}, m_{2}}^{\prime}$ admits $\bar{\rho}$.)

- The representation $\sigma_{m_{1}, m_{2}}^{\prime}$ with $m_{1} \neq m_{2}$ simply admits $\bar{\rho}$ if either

$$
\left.\bar{\rho}\right|_{I_{\ell}} \sim\left(\begin{array}{cc}
\omega^{1-m_{1}} & * \\
0 & \omega^{-m_{2}}
\end{array}\right)
$$

or

$$
\left.\bar{\rho}\right|_{I_{\ell}} \sim\left(\begin{array}{cc}
\omega^{1-m_{2}} & * \\
0 & \omega^{-m_{1}}
\end{array}\right) .
$$

- The representation $\sigma_{m, m}^{\prime}$ simply admits $\bar{\rho}$ if

$$
\left.\bar{\rho}\right|_{I_{\ell}} \sim\left(\begin{array}{cc}
\omega^{1-m} & * \\
0 & \omega^{-m}
\end{array}\right)
$$

is très ramifié.

- The representation $\sigma_{\left\{m_{1}, m_{2}\right\}, a}^{\prime}$ with $m_{1} \neq m_{2}$ admits $\bar{\rho}$ if either $\sigma_{m_{1}, m_{2}}^{\prime}$ or $\sigma_{m_{2}, m_{1}}^{\prime}$ admits $\bar{\rho}$ and if $\left.\left(\omega^{-1} \operatorname{det} \bar{\rho}\right)\right|_{W_{\mathbf{Q}_{\ell}}}$ equals the central character of $\sigma_{\left\{m_{1}, m_{2}\right\}, a}^{\prime}$. (Note that in this case $\left.\sigma_{\left\{m_{1}, m_{2}\right\}, a}^{\prime}\right|_{U_{0}(\ell)}=\sigma_{m_{1}, m_{2}}^{\prime} \oplus \sigma_{m_{2}, m_{1}}^{\prime}$.)

- The representation $\sigma_{\left\{m_{1}, m_{2}\right\}, a}^{\prime}$ with $m_{1} \neq m_{2}$ simply admits $\bar{\rho}$ if $\left.\left(\omega^{-1} \operatorname{det} \bar{\rho}\right)\right|_{W_{\mathbf{Q}_{\ell}}}$ equals the central character of $\sigma_{\left\{m_{1}, m_{2}\right\}, a}^{\prime}$ and either

$$
\left.\bar{\rho}\right|_{I_{\ell}} \sim\left(\begin{array}{cc}
\omega^{1-m_{1}} & * \\
0 & \omega^{-m_{2}}
\end{array}\right)
$$

or

$$
\left.\bar{\rho}\right|_{I_{\ell}} \sim\left(\begin{array}{cc}
\omega^{1-m_{2}} & * \\
0 & \omega^{-m_{1}}
\end{array}\right) .
$$


- The representation $\sigma_{\{m\}, a}^{\prime}$ admits $\bar{\rho}$ if

- $\sigma_{m, m}^{\prime}$ admits $\bar{\rho}$,

- $\left.\left(\omega^{-1} \operatorname{det} \bar{\rho}\right)\right|_{W_{\mathbf{Q}_{\ell}}}$ equals the central character of $\sigma_{\{m\}, a}^{\prime}$,

- and, if

$$
\left.\bar{\rho}\right|_{I_{\ell}} \sim\left(\begin{array}{cc}
\omega^{1-m} & * \\
0 & \omega^{-m}
\end{array}\right)
$$

is très ramifié, then

$$
\bar{\rho} \sim\left(\begin{array}{cc}
* & * \\
0 & \omega^{-m} \chi
\end{array}\right)
$$

where $\chi$ is unramified and sends Frobenius to $-a$.

(Note that $\left.\sigma_{\{m\}, a}^{\prime}\right|_{U_{0}(\ell)}=\sigma_{m, m}^{\prime}$.)

- The representation $\sigma_{\{m\}, a}^{\prime}$ simply admits $\bar{\rho}$ if $\sigma_{\{m\}, a}^{\prime}$ admits $\bar{\rho}$.

We remark that the definition of " $\sigma$ admits the Cartier dual of $\vec{\rho}$ " might look more natural to the reader. We are forced to adopt this version of the definition by some unfortunate choices of normalisations in CDT.

We say that a reducible $\ell$-type $\tau$ (resp. irreducible $\ell$-type $\tau$, resp. extended $\ell$ type $\tau^{\prime}$ with $\left.\tau^{\prime}\right|_{I_{\ell}}$ irreducible) admits a continuous representation $\bar{\rho}: G_{\ell} \rightarrow \mathrm{GL}_{2}\left(\overline{\mathbf{F}}_{\ell}\right)$ if $\tau$ (resp. $\tau$, resp. $\tau^{\prime}$ ) admits an irreducible representation of $\mathrm{GL}_{2}\left(\mathbf{Z}_{\ell}\right)$ (resp. $U_{0}(\ell)$, resp. $\widetilde{U}_{0}(\ell)$ ) over $\overline{\mathbf{F}}_{\ell}$ which in turn admits $\bar{\rho}$. We say that $\tau$ (resp. $\tau$, resp. $\tau^{\prime}$ ) simply admits $\bar{\rho}$ if

- $\tau$ (resp. $\tau$, resp. $\tau^{\prime}$ ) admits a unique irreducible representation $\sigma$ of $\mathrm{GL}_{2}\left(\mathbf{Z}_{\ell}\right)$ (resp. $U_{0}(\ell)$, resp. $\widetilde{U}_{0}(\ell)$ ) over $\overline{\mathbf{F}}_{\ell}$ which admits $\bar{\rho}$,

- $\tau$ (resp. $\tau$, resp. $\tau^{\prime}$ ) simply admits $\sigma$,

- and $\sigma$ simply admits $\bar{\rho}$.

Note that the concept of "simply admits" is strictly stronger than the concept "admits".

The starting point for this work was the following conjecture, of which a few examples will be verified in $\$ 2.1$

Conjecture 1.3.1. Let $k$ be a finite subfield of $\overline{\mathbf{F}}_{\ell}, \bar{\rho}: G_{\ell} \rightarrow \mathrm{GL}_{2}(k)$ a continuous representation, $\tau$ an $\ell$-type and $\tau^{\prime}$ an extended $\ell$-type with irreducible restriction to $I_{\ell}$. Suppose that $\operatorname{det} \tau$ and $\operatorname{det} \tau^{\prime}$ are tamely ramified, that the centraliser of the image of $\bar{\rho}$ is $k$ and that the image of $\tau$ is not contained in the centre of $\mathrm{GL}_{2}\left(\overline{\mathbf{Q}}_{\ell}\right)$.

(1) $\tau$ (resp. $\left.\tau^{\prime}\right)$ admits $\bar{\rho}$ if and only if $R_{V, \mathcal{O}}^{D} \neq(0)\left(\right.$ resp. $\left.R_{V, \mathcal{O}}^{D^{\prime}} \neq(0)\right)$, i.e. if and only if there is a finite extension $K^{\prime}$ of $\mathbf{Q}_{\ell}$ in $\overline{\mathbf{Q}}_{\ell}$ and a continuous representation $\rho: G_{\ell} \rightarrow \mathrm{GL}_{2}\left(\mathcal{O}_{K^{\prime}}\right)$ which reduces to $\bar{\rho}$ and has type $\tau$ (resp. has extended type $\left.\tau^{\prime}\right)$.

(2) $\tau$ (resp. $\left.\tau^{\prime}\right)$ simply admits $\bar{\rho}$ if and only if $\tau$ (resp. $\left.\tau^{\prime}\right)$ is acceptable for $\bar{\rho}$.

We remark that to check if $\tau$ or $\tau^{\prime}$ simply admits $\bar{\rho}$ is a relatively straightforward computation. On the other hand to show that $\tau$ or $\tau^{\prime}$ is acceptable for $\bar{\rho}$ is at present a non-trivial undertaking. (The reader who doubts us might like to compare $\S[$ with \$4, \$5 \$6, \$7] \$8 and \$9] All the latter sections are devoted to verifying some very special cases of this conjecture.) 
1.4. The main theorems. With these definitions, we can state our two main theorems. The proofs very closely parallel the proof of Theorem 7.1.1 of [CDT].

Theorem 1.4.1. Let $\ell$ be an odd prime, $K$ a finite extension of $\mathbf{Q}_{\ell}$ in $\overline{\mathbf{Q}}_{\ell}$ and $k$ the residue field of $K$. Let

$$
\rho: G_{\mathbf{Q}} \longrightarrow \mathrm{GL}_{2}(K)
$$

be an odd continuous representation ramified at only finitely many primes. Assume that its reduction

$$
\bar{\rho}: G_{\mathbf{Q}} \longrightarrow \mathrm{GL}_{2}(k)
$$

is absolutely irreducible after restriction to $\mathbf{Q}\left(\sqrt{(-1)^{(\ell-1) / 2} \ell}\right)$ and is modular. Further, suppose that

- $\left.\bar{\rho}\right|_{G_{\ell}}$ has centraliser $k$,

- $\left.\rho\right|_{G_{\ell}}$ is potentially Barsotti-Tate with $\ell$-type $\tau$,

- $\tau$ admits $\bar{\rho}$,

- and $\tau$ is weakly acceptable for $\bar{\rho}$.

Then $\rho$ is modular.

Proof. Note that the existence of $\rho$ shows that $\tau$ is acceptable for $\bar{\rho}$. Now the proof is verbatim the proof of Theorem 7.1 .1 of [CDT] (see $\S 1.3, \S 1.4, \S 3, \S 4, \S 5$ and $\S 6$ of that paper, and the corrigendum at the end of this paper), with the following exceptions.

- On page 539 one should take $U_{S, \ell}=U_{\tau}, V_{S, \ell}=\operatorname{ker} \sigma_{\tau}$ and $\sigma_{S, l}=\sigma_{\tau}$.

- In the proof of Lemma 5.1.1 one must use Lemma 1.2.1 of this paper, in addition to the results recalled in $\S 4$ of $[\mathrm{CDT}$.

- On page 546 replace "Setting $S=T(\bar{\rho}) \cup\{r\}$..." to the end of the first paragraph by the following. (Again the key component of this argument is very similar to the main idea of $[\mathrm{Kh}$.)

"Set $S=T(\bar{\rho}) \cup\{r\} ; U_{S}^{\prime}=\prod_{p} U_{S, p}^{\prime}$ where $U_{S, p}^{\prime}=U_{1}\left(p^{c_{p}}\right)$ if $p \in T(\bar{\rho})$ and $U_{S, p}^{\prime}=U_{S, p}$ otherwise; $V_{S}^{\prime}=\prod_{p} V_{S, p}^{\prime}$ where $V_{S, p}^{\prime}=U_{1}\left(p^{c_{p}}\right)$ if $p \in T(\bar{\rho})$ and $V_{S, p}^{\prime}=V_{S, p}$ otherwise; and $L_{S}^{\prime}=\operatorname{Hom}_{\mathcal{O}\left[U_{S}^{\prime} / V_{S}^{\prime}\right]}\left(M_{\ell}, H^{1}\left(X_{V_{S}^{\prime}}, \mathcal{O}\right)\right)\left[I_{S}^{\prime}\right]$. Then $\Gamma=\mathrm{SL}_{2}(\mathbf{Z}) \cap\left(U_{S}^{\prime} \mathrm{GL}_{2}\left(\mathbf{Z}_{\ell}\right)\right)$ satisfies the hypotheses of Theorem 6.1.1. Furthermore

$$
H^{1}\left(Y_{U_{S}^{\prime} \mathrm{GL}_{2}\left(\mathbf{Z}_{\ell}\right)}, \mathcal{F}_{M}\right) \cong H^{1}\left(\Gamma, L_{n} \otimes k\right)
$$

as a $\widetilde{\mathbf{T}}_{S}^{\prime}$-module, where $M$ is the module for $U_{S, \ell}=\mathrm{GL}_{2}\left(\mathbf{Z}_{\ell}\right)$ defined by the action of $\mathrm{GL}_{2}\left(\mathbf{F}_{\ell}\right)$ on $L_{n} \otimes k$. Therefore $\mathfrak{m}_{S}$ is in the support of $H^{1}\left(Y_{U_{S}^{\prime} \mathrm{GL}_{2}\left(\mathbf{Z}_{\ell}\right)}, \mathcal{F}_{M}\right)$.

We now drop the special assumption on $\left.\bar{\rho}\right|_{I_{\ell}}$ made in the last paragraph. Twisting we see that if $\sigma$ is an irreducible representation of $\mathrm{GL}_{2}\left(\mathbf{Z}_{\ell}\right)$ over $\overline{\mathbf{F}}_{\ell}$ admitting $\left.\bar{\rho}\right|_{G_{\ell}}$, then

$$
H^{1}\left(Y_{U_{S}^{\prime} \mathrm{GL}_{2}\left(\mathbf{Z}_{\ell}\right)}, \mathcal{F}_{\sigma^{\vee}}\right)_{\mathfrak{m}_{S}} \neq(0) .
$$

Moreover if $\tau$ is irreducible and if $\sigma^{\prime}$ is an irreducible representation of $U_{0}(\ell)$ over $\overline{\mathbf{F}}_{\ell}$ which admits $\left.\bar{\rho}\right|_{G_{\ell}}$, then we see using the definition of admits and Lemmas 3.1.1 and 6.1.2 of [CDT] that

$$
H^{1}\left(Y_{U_{S}^{\prime}}, \mathcal{F}_{\sigma^{\vee}}\right)_{\mathfrak{m}_{S}} \cong H^{1}\left(Y_{U_{S}^{\prime} \mathrm{GL}_{2}\left(\mathbf{z}_{\ell}\right)}, \mathcal{F}_{\operatorname{Ind}_{U_{0}(\ell)}^{\mathrm{GL}_{2}\left(\mathbf{z}_{\ell)}\right)} \sigma^{\vee}}\right)_{\mathfrak{m}_{S}} \neq(0)
$$


It follows from the definition of admits and Lemma 6.1.2 of [CDT] that $\mathfrak{m}_{S}$ is in the support of $H^{1}\left(Y_{U_{S}^{\prime}}, \mathcal{F}_{\mathrm{Hom}_{\mathcal{O}}\left(M_{\ell}, \mathcal{O}\right)}\right)$, so $L_{S}^{\prime}$ is non-zero. Using the fact that Lemma 5.1.1 holds with $U_{S}^{\prime}$ replacing $U_{S}$ and $\sigma_{\ell}$ replacing $\sigma_{S}$ and the discussion on page 541 we conclude that $\mathcal{N}_{S}$ is non-empty."

Theorem 1.4.2. Let $\ell$ be an odd prime, $K$ a finite extension of $\mathbf{Q}_{\ell}$ in $\overline{\mathbf{Q}}_{\ell}$ and $k$ the residue field of $K$. Let

$$
\rho: G_{\mathbf{Q}} \longrightarrow \mathrm{GL}_{2}(K)
$$

be an odd continuous representation ramified at only finitely many primes. Assume that its reduction

$$
\bar{\rho}: G_{\mathbf{Q}} \longrightarrow \mathrm{GL}_{2}(k)
$$

is absolutely irreducible after restriction to $\mathbf{Q}\left(\sqrt{(-1)^{(\ell-1) / 2} \ell}\right)$ and is modular. Further, suppose that

- $\left.\bar{\rho}\right|_{G_{\ell}}$ has centraliser $k$,

- $\left.\rho\right|_{G_{\ell}}$ is potentially Barsotti-Tate with extended $\ell$-type $\tau^{\prime}$,

- $\tau^{\prime}$ admits $\bar{\rho}$,

- and $\tau^{\prime}$ is weakly acceptable for $\bar{\rho}$.

Then $\rho$ is modular.

Proof. The existence of $\rho$ shows that $\tau^{\prime}$ is in fact acceptable for $\bar{\rho}$. Again the proof now follows very closely that of Theorem 7.1.1 of [CDT]. In this case we have to make the following changes. All references are to [CDT] unless otherwise indicated.

- On page 539 one should take $U_{S, \ell}=U_{0}(\ell), V_{S, \ell}=\left.\operatorname{ker} \sigma_{\tau^{\prime}}\right|_{U_{0}(\ell)}$ and $\sigma_{S, \ell}=$ $\left.\sigma_{\tau^{\prime}}\right|_{U_{0}(\ell)}$. One should also define $\widetilde{U}_{S}$ to be the group generated by $U_{S}$ and $w_{\ell} \in \mathrm{GL}_{2}\left(\mathbf{Q}_{\ell}\right)$ and $\widetilde{\sigma}_{S}$ to be the extension of $\sigma_{S}$ to $\widetilde{U}_{S}$ which restricts to $\sigma_{\tau^{\prime}}$ on $\widetilde{U}_{0}(\ell)$.

- In the statement of Lemma 5.1.1 one should replace $\operatorname{Hom}_{U_{S}}\left(\sigma_{S}, \pi^{\infty}\right)$ by $\operatorname{Hom}_{\widetilde{U}_{S}}\left(\widetilde{\sigma}_{S}, \pi^{\infty}\right)$.

- In the proof of Lemma 5.1.1 one must use Lemma11.2.1 above in addition to the results recalled in $\S 4$ of $[\mathrm{CDT}$.

- Because $\tau^{\prime}$ is acceptable for $\bar{\rho}$, we know that $\operatorname{det} \tau$ of a Frobenius lift is $\ell \zeta$ for some root of unity $\zeta$. Thus, $\sigma_{\tau^{\prime}}\left(\ell^{s}\right)=1$ for some $s>0$. Hence, $\widetilde{\sigma}_{S}$ factors through the finite group $\widetilde{G}_{S}=\widetilde{U}_{S} / V_{S} \ell^{\ell \mathbf{Z}}$, where $\ell \in \mathrm{GL}_{2}\left(\mathbf{Q}_{\ell}\right)$.

- In $\S 5.3$ choose $M_{\ell}$ so that it is invariant for the action of $\widetilde{U}_{0}(\ell) / V_{S, \ell} \ell^{s \mathbf{Z}}$. Also, in the definition of $L_{S}$ replace $G_{S}$ by $\widetilde{G}_{S}$.

- In the proof of Lemma 5.3.1 replace $U_{S}$ by $\widetilde{U}_{S}$ and $\sigma_{S}$ by $\widetilde{\sigma}_{S}$.

- Note that $w_{\ell}$ acts naturally on $Y_{S}$ and $\mathcal{F}_{S}$. In Lemma 6.1 .3 we should replace the group $H_{c}^{1}\left(Y_{S}, \mathcal{F}_{S}\right)$ by $H_{c}^{1}\left(Y_{S}, \mathcal{F}_{S}\right)^{w_{\ell}=1}$ and the group $H^{1}\left(Y_{S}, \mathcal{F}_{S}\right)$ by $H^{1}\left(Y_{S}, \mathcal{F}_{S}\right)^{w_{\ell}=1}$.

- Replace $\S 6.2$ with the proof of the required extension of Proposition 5.4.1 given below.

- On page 547 the isomorphism

$$
H_{c}^{1}\left(Y_{S}, \mathcal{F}_{S}\right) \longrightarrow \operatorname{Hom}_{\mathcal{O}}\left(H^{1}\left(Y_{S}, \mathcal{F}_{S}\right), \mathcal{O}\right)
$$

on line 6 is $\tilde{\mathbf{T}}_{S}^{\prime}\left[w_{l}\right]$-linear. In the next line one should not only localise at $\mathfrak{m}$ but restrict to the kernel of $w_{\ell}-1$. Because $w_{\ell}^{2}=1$ on $H^{1}\left(Y_{S}, \mathcal{F}_{S}\right)_{\mathfrak{m}}$ we see 
that the natural map

$$
H^{1}\left(Y_{S}, \mathcal{F}_{S}\right)_{\mathfrak{m}}^{w_{\ell}=1} \longrightarrow H^{1}\left(Y_{S}, \mathcal{F}_{S}\right)_{\mathfrak{m}} /\left(w_{\ell}-1\right)
$$

is an isomorphism, and so the map

$$
L_{S} \longrightarrow \operatorname{Hom}_{\mathcal{O}}\left(L_{S}, \mathcal{O}\right)
$$

is also an isomorphism.

- On page 547 the groups $H^{1}\left(Y_{S}, \mathcal{F}_{S}\right)_{\mathfrak{m}_{S}^{(p)}}$ and $H^{1}\left(Y_{S^{\prime}}, \mathcal{F}_{S^{\prime}}\right)_{\mathfrak{m}_{S}^{(p)}}$ should be replaced by their maximal subgroups on which $w_{\ell}=1$.

- On page 549 one should also define $\widetilde{V}_{0}$ (resp. $\widetilde{V}_{1}$ ) to be the group generated by $V_{0}$ (resp. $\left.V_{1}\right)$ and $w_{\ell} \in \mathrm{GL}_{2}\left(\mathbf{Q}_{\ell}\right)$. Similarly define $\widetilde{\sigma}$ to be $\widetilde{\sigma}_{\emptyset} \otimes \psi_{r^{\prime}}^{-2}$.

- In Lemma 6.4 .1 replace $V_{0}$ by $\widetilde{V}_{0}, V_{1}$ by $\widetilde{V}_{1}$ and $\sigma$ by $\widetilde{\sigma}$. In the proof of Lemma 6.4.1 also replace $U_{\left\{r, r^{\prime}\right\}}$ (resp. $\left.U_{S \cup\left\{r, r^{\prime}\right\}}\right)$ by $\widetilde{U}_{\left\{r, r^{\prime}\right\}}\left(\operatorname{resp} . \widetilde{U}_{S \cup\left\{r, r^{\prime}\right\}}\right)$ and $\sigma_{\left\{r, r^{\prime}\right\}}\left(\operatorname{resp} . \sigma_{S \cup\left\{r, r^{\prime}\right\}}\right)$ by $\widetilde{\sigma}_{\left\{r, r^{\prime}\right\}}\left(\operatorname{resp} . \widetilde{\sigma}_{S \cup\left\{r, r^{\prime}\right\}}\right)$.

- On line 20 of page $550 M$ should be chosen as a model of $\widetilde{\sigma}$. This is possible because ker $\widetilde{\sigma}$ has finite index in $\widetilde{V}_{0}$, because in turn $\sigma_{\tau^{\prime}}\left(\ell^{s}\right)=1$ for some $s>0$. One should also set $L_{i}=H^{1}\left(Y_{V_{i}}, \mathcal{F}_{M^{\vee}}\right)_{\mathfrak{m}}^{w_{\ell}=1}$. On line 25 , we must replace $V_{i}$ by $\widetilde{V}_{i}$.

- In the proof of Lemma 6.4 .2 , one must replace $V_{1}$ by $\widetilde{V}_{1}$ and $\sigma$ by $\widetilde{\sigma}$.

- In line 2 of the proof of Lemma 6.4.3, to see that $L_{1}$ is a direct summand of $H^{1}\left(Y_{V_{1}}, \mathcal{F}_{M \vee}\right)$ as an $\mathcal{O}\left[\Delta_{S}\right]$-module, one needs to note that $H^{1}\left(Y_{V_{1}}, \mathcal{F}_{M \vee}\right)_{\mathfrak{m}}^{w_{\ell}=1}$ is a direct summand of $H^{1}\left(Y_{V_{1}}, \mathcal{F}_{M^{\vee}}\right)_{\mathfrak{m}}$, because $w_{\ell}^{2}=1$ on $H^{1}\left(Y_{V_{1}}, \mathcal{F}_{M^{\vee}}\right)_{\mathfrak{m}}$.

- On line 12 of page 551 replace $R_{V, \mathcal{O}}^{\emptyset, D}$ by $R_{V, \mathcal{O}}^{\emptyset, D^{\prime}}$.

Proof of extension of Proposition 5.4.1 of [CDT]. Let $\Theta=\bigotimes_{p \in T(\bar{\rho})} M_{p}$.

First suppose that $\tau^{\prime}$ admits $\sigma_{\left\{m_{1}, m_{2}\right\}, a}^{\prime}$ with $m_{1} \neq m_{2}$ and that $\sigma_{\left\{m_{1}, m_{2}\right\}, a}^{\prime}$ admits $\bar{\rho}$. As in the proof of Theorem 1.4.1 (especially $\S 6.2$ of [CDT] as modified above), we have

$$
H^{1}\left(Y_{\{r\}}, \mathcal{F}_{\Theta \vee} \otimes \mathcal{F}_{\left(\sigma_{m_{1}, m_{2}}^{\prime}\right) \vee}\right)_{\mathfrak{m}_{\{r\}}^{\prime}} \neq(0)
$$

On the other hand

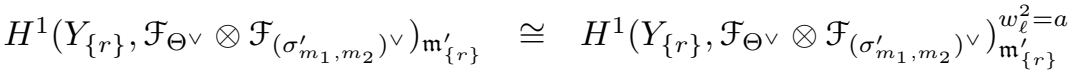

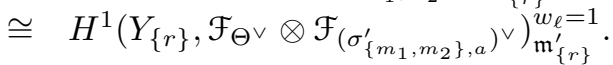

Thus, using the definition of "admits" and Lemma 6.1.2 of [CDT], we see that

$$
H^{1}\left(Y_{\{r\}}, \mathcal{F}_{\{r\}}\right)_{\mathfrak{m}_{\{r\}}^{\prime}}^{w_{\ell}=1} \neq(0),
$$

so $\mathcal{N}_{\emptyset}=\mathcal{N}_{\{r\}} \neq \emptyset$.

Next suppose that $\tau^{\prime}$ admits $\sigma_{\{m\}, a}^{\prime}$ which in turn admits $\bar{\rho}$. Assume that $\left.\bar{\rho}\right|_{G_{\ell}}$ is irreducible or peu ramifié. By twisting we may reduce to the case $m=0$. As in the proof of Theorem 1.4.1 (especially $\S 6.2$ of [CDT] as modified above), we have

$$
H^{1}\left(Y_{U_{\{r\}} \mathrm{GL}_{2}\left(\mathbf{Z}_{\ell}\right)}, \mathcal{F}_{\Theta^{\vee}}\right)_{\mathfrak{m}_{\{r\}}^{\prime}} \neq(0)
$$

Thus

$$
H^{1}\left(Y_{U_{\{r\}} \mathrm{GL}_{2}\left(\mathbf{Z}_{\ell}\right)}, \mathcal{F}_{\Theta^{\vee}}\right)_{\mathfrak{m}_{\ell r\}}^{\prime}}^{w_{2}^{2}=\widetilde{a}^{2}} \neq(0),
$$

where $\widetilde{a}$ is the Teichmüller lift of $a$. Using the embedding

$$
\widetilde{a}+w_{\ell}: H^{1}\left(Y_{U_{\{r\}} \mathrm{GL}_{2}\left(\mathbf{Z}_{\ell}\right)}, \mathcal{F}_{\Theta^{\vee}}\right) \otimes \overline{\mathbf{Q}}_{\ell} \hookrightarrow H^{1}\left(Y_{\{r\}}, \mathcal{F}_{\Theta \vee}\right) \otimes \overline{\mathbf{Q}}_{\ell},
$$


we deduce that

$$
H^{1}\left(Y_{\{r\}}, \mathcal{F}_{\Theta^{\vee}}\right)_{\mathfrak{m}_{\{r\}}^{\prime}}^{w_{\ell}=\widetilde{a}} \neq(0)
$$

and so

$$
H^{1}\left(Y_{\{r\}}, \mathcal{F}_{\Theta \vee} \otimes \mathcal{F}_{\left(\sigma_{\{0\}, a}^{\prime}\right)^{\vee}}\right)_{\mathfrak{m}_{\ell}^{\prime}=1}^{w_{\{r\}}} \neq(0) .
$$

Thus, using the definition of "admits" and Lemma 6.1.2 of [CDT], we see that

$$
H^{1}\left(Y_{\{r\}}, \mathcal{F}_{\{r\}}\right)_{\mathfrak{m}_{\{r\}}^{\prime}}^{w_{\ell}^{\prime}=1} \neq(0)
$$

and so $\mathcal{N}_{\emptyset}=\mathcal{N}_{\{r\}} \neq \emptyset$.

Finally suppose that $\tau^{\prime}$ admits $\sigma_{\{m\}, a}$ which in turn admits $\bar{\rho}$, and that $\left.\bar{\rho}\right|_{G_{\ell}}$ is reducible and très ramifié. By twisting we may reduce to the case $m=0$. Note that $\bar{\rho}_{I_{\ell}}\left(\right.$ Frob $\left._{\ell}\right)=-a$. As in the proof of Theorem 1.4.1 (especially $\S 6.2$ of [CDT] as modified above), we have

$$
H^{1}\left(Y_{\{r\}}, \mathcal{F}_{\Theta^{\vee}}\right)_{\mathfrak{m}_{\{r\}}^{\prime}} \neq(0) .
$$

Suppose that $\pi$ is a cuspidal automorphic representation which contributes to $H^{1}\left(Y_{\{r\}}, \mathcal{F}_{\Theta^{\vee}}\right)_{\mathfrak{m}^{\prime}}{ }_{\{r\}}$, so $\pi$ is a cuspidal automorphic representation of $\mathrm{GL}_{2}(\mathbf{A})$ such that $\pi_{\infty}$ is the lowest discrete series with trivial infinitesimal character, $\rho_{\pi}$ is a lift of $\bar{\rho}$ of type $(\{r\}, 1)$, and hence of type $(\emptyset, 1)$, and $\operatorname{dim} \pi_{\ell}^{U_{0}(\ell)}=1$. As $\epsilon^{-1} \operatorname{det} \rho_{\pi}$ has order prime to $\ell$, we see that $w_{\ell}^{2}$ acts on $\pi_{\ell}^{U_{0}(\ell)}$ by the Teichmüller lift of $a^{2}$. As $\pi_{\ell}$ has a $U_{0}(\ell)$-fixed vector but no $\mathrm{GL}_{2}\left(\mathbf{Z}_{\ell}\right)$-fixed vector, we see that $1+U_{\ell} w_{\ell}^{-1}=0$ on $\pi_{\ell}^{U_{0}(\ell)}$. On the other hand, the eigenvalue of $U_{\ell}$ on $\pi_{\ell}^{U_{0}(\ell)}$ reduces to $-a$. Thus, $w_{\ell}$ acts on $\pi_{\ell}^{U_{0}(\ell)}$ by the Teichmüller lift of $a$, so $w_{\ell}$ acts on $H^{1}\left(Y_{\{r\}}, \mathcal{F}_{\Theta^{\vee}}\right)_{\mathfrak{m}_{\{r\}}^{\prime}}$ by the Teichmüller lift of $a$. We deduce that

$$
H^{1}\left(Y_{\{r\}}, \mathcal{F}_{\Theta \vee} \otimes \mathcal{F}_{\left(\sigma_{0,0}^{\prime}\right)^{\vee}}\right)_{\mathfrak{m}_{\{r\}}^{\prime}}^{w_{\ell}=a} \neq(0) .
$$

Using the definition of "admits" and Lemma 6.1.2 of [CDT], we see that

$$
H^{1}\left(Y_{\{r\}}, \mathcal{F}_{\{r\}}\right)_{\mathfrak{m}_{\{r\}}^{\prime}}^{w_{\ell}^{\prime}=1} \neq(0)
$$

so $\mathcal{N}_{\emptyset}=\mathcal{N}_{\{r\}} \neq \emptyset$.

\section{EXAMPLES AND APPLiCATIONS}

2.1. Examples. Now we will specialise to the case $\ell=3$. Fix an element $\zeta \in$ $\mathrm{GL}_{2}\left(\mathbf{Z}_{3}\right)$ with $\zeta^{3}=1$ but $\zeta \neq 1$. The following definitions, which concern isomorphism classes of 2-dimensional representations into $\mathrm{GL}_{2}\left(\overline{\mathbf{Q}}_{3}\right)$, do not depend on this choice. We will consider the following $\ell$-types. (These are in fact, up to twist, a complete list of the wildly ramified types which can arise from elliptic curves over $\mathbf{Q}_{3}$, or, in the case of conductor 243 , the extended types. We will not need this fact. Rather the justification for studying these particular types can be found in $\$ 2.2$. More detailed information about the fixed fields of these types can be found in $₫[6$ )

- $\tau_{1}$ corresponds to the order 3 homomorphism

$$
\mathbf{Z}_{3}^{\times} \longrightarrow \mathbf{Z}_{3}[\zeta]^{\times}
$$


determined by

$$
\begin{aligned}
-1 & \longmapsto 1 \\
4 & \longmapsto \zeta .
\end{aligned}
$$

- $\tau_{-1}$ corresponds to the order 3 homomorphism

$$
\mathbf{Z}_{3}[\sqrt{-1}]^{\times} \longrightarrow \mathbf{Z}_{3}[\zeta]^{\times}
$$

determined by

$$
\begin{aligned}
\sqrt[4]{-1} & \longmapsto 1 \\
4 & \longmapsto 1 \\
1+3 \sqrt{-1} & \longmapsto \zeta .
\end{aligned}
$$

- $\tau_{3}$ is the unique 3-type such that $\left.\tau_{3}\right|_{I_{\mathbf{Q}_{3}(\sqrt{3})}}$ corresponds to the order 6 homomorphism

$$
\mathbf{Z}_{3}[\sqrt{3}]^{\times} \longrightarrow \mathbf{Z}_{3}[\zeta]^{\times}
$$

determined by

$$
\begin{array}{rll}
-1 & \longmapsto & -1 \\
4 & \longmapsto 1 \\
1+\sqrt{3} & \longmapsto \zeta .
\end{array}
$$

- $\tau_{-3}$ is the unique 3-type such that $\left.\tau_{-3}\right|_{I_{\mathbf{Q}_{3}(\sqrt{-3})}}$ corresponds to the order 6 homomorphism

$$
\mathbf{Z}_{3}[\sqrt{-3}]^{\times} \longrightarrow \mathbf{Z}_{3}[\zeta]^{\times}
$$

determined by

$$
\begin{aligned}
-1 & \longmapsto-1 \\
4 & \longmapsto 1 \\
1+3 \sqrt{-3} & \longmapsto 1 \\
1+\sqrt{-3} & \longmapsto \zeta .
\end{aligned}
$$

For $i \in \mathbf{Z} / 3 \mathbf{Z}$, we will also consider the unique extended 3-types $\tau_{i}^{\prime}$ whose restrictions to $G_{\mathbf{Q}_{3}(\sqrt{-3})}$ correspond to the homomorphisms

$$
\mathbf{Q}_{3}(\sqrt{-3})^{\times} \rightarrow \mathbf{Q}_{3}(\zeta)^{\times}
$$

determined by

$$
\begin{aligned}
\sqrt{-3} & \longmapsto \zeta-\zeta^{-1} \\
-1 & \longmapsto-1 \\
4 & \longmapsto 1 \\
1+3 \sqrt{-3} & \longmapsto \zeta \\
1+\sqrt{-3} & \longmapsto \zeta^{i} .
\end{aligned}
$$

Subsequent sections of this paper will be devoted to checking the following special cases of Conjecture 1.3.1.

Lemma 2.1.1. Suppose that $\bar{\rho}: G_{3} \rightarrow \mathrm{GL}_{2}\left(\mathbf{F}_{3}\right)$ and

$$
\left.\bar{\rho}\right|_{I_{3}} \sim\left(\begin{array}{cc}
1 & * \\
0 & \omega
\end{array}\right)
$$

is très ramifié. Both $\tau_{1}$ and $\tau_{-1}$ simply admit $\bar{\rho}$. 
Theorem 2.1.2. Suppose that $\bar{\rho}: G_{3} \rightarrow \mathrm{GL}_{2}\left(\mathbf{F}_{3}\right)$ and

$$
\left.\bar{\rho}\right|_{I_{3}} \sim\left(\begin{array}{cc}
1 & * \\
0 & \omega
\end{array}\right)
$$

is très ramifié. Both $\tau_{1}$ and $\tau_{-1}$ are weakly acceptable for $\bar{\rho}$.

Lemma 2.1.3. Suppose that $\bar{\rho}: G_{3} \rightarrow \mathrm{GL}_{2}\left(\mathbf{F}_{3}\right)$ and

$$
\left.\bar{\rho}\right|_{I_{3}} \sim\left(\begin{array}{cc}
\omega & * \\
0 & 1
\end{array}\right)
$$

is très ramifié. Both $\tau_{3}$ and $\tau_{-3}$ simply admit $\bar{\rho}$.

Theorem 2.1.4. Suppose that $\bar{\rho}: G_{3} \rightarrow \mathrm{GL}_{2}\left(\mathbf{F}_{3}\right)$ and

$$
\left.\bar{\rho}\right|_{I_{3}} \sim\left(\begin{array}{cc}
\omega & * \\
0 & 1
\end{array}\right)
$$

is très ramifié. Both $\tau_{3}$ and $\tau_{-3}$ are weakly acceptable for $\bar{\rho}$.

Lemma 2.1.5. Let $i \in \mathbf{Z} / 3 \mathbf{Z}$. Suppose that $\bar{\rho}: G_{3} \rightarrow \mathrm{GL}_{2}\left(\mathbf{F}_{3}\right)$ and

$$
\bar{\rho} \sim\left(\begin{array}{cc}
\omega & * \\
0 & 1
\end{array}\right)
$$

is très ramifié. The extended 3-type $\tau_{i}^{\prime}$ simply admits $\bar{\rho}$.

Theorem 2.1.6. Let $i \in \mathbf{Z} / 3 \mathbf{Z}$. Suppose that $\bar{\rho}: G_{3} \rightarrow \mathrm{GL}_{2}\left(\mathbf{F}_{3}\right)$ and

$$
\bar{\rho} \sim\left(\begin{array}{cc}
\omega & * \\
0 & 1
\end{array}\right)
$$

is très ramifié. Then $\tau_{i}^{\prime}$ is weakly acceptable for $\bar{\rho}$.

We remark that in Theorems 2.1.2 2.1.4 and 2.1.6 we could replace "weakly acceptable" by "acceptable". This can be shown by using elliptic curves to construct explicit liftings of the desired type. For Theorems 2.1.2 and 2.1.4 the results of $\mathrm{Man}$ suffice for this. For Theorem 2.1.6 a slightly more refined analysis along the lines of $\$ 2.3$ is required.

We also remark that it was Lemmas 2.1.1 2.1.3, 2.1.5 and Conjecture 1.3.1 which originally suggested to us that we try to prove Theorems 2.1.2, 2.1.4 and 2.1.6.

2.2. Applications. Conditional on the results stated in 2.1 which we will prove below, we prove the following results.

Theorem 2.2.1. Any continuous absolutely irreducible representation $\bar{\rho}: G_{\mathbf{Q}} \rightarrow$ $\mathrm{GL}_{2}\left(\mathbf{F}_{5}\right)$ with cyclotomic determinant is modular.

Proof. Choose an element $\bar{\zeta} \in \mathrm{GL}_{2}\left(\mathbf{F}_{5}\right)$ with $\bar{\zeta}^{3}=1$ but $\bar{\zeta} \neq 1$. (The following classification will be independent of the choice of $\bar{\zeta}$.) Then up to equivalence and twisting by a quadratic character, one of the following possibilities can be attained.

(1) $\bar{\rho}$ is tamely ramified at 3 .

(2) $\left.\bar{\rho}\right|_{G_{3}}$ is given by the character

$$
\mathbf{Q}_{3}^{\times} \longrightarrow \mathbf{F}_{5}(\bar{\zeta})^{\times}
$$


determined by

$$
\begin{aligned}
3 & \longmapsto \bar{\zeta}^{i}\left(\bar{\zeta}-\bar{\zeta}^{-1}\right) \\
-1 & \longmapsto \overline{1} \\
4 & \longmapsto \bar{\zeta}
\end{aligned}
$$

where $i \in \mathbf{Z} / 3 \mathbf{Z}$.

(3) $\left.\bar{\rho}\right|_{G_{\mathbf{Q}_{3}(\sqrt{-1})}}$ is given by the character

determined by

$$
\mathbf{Q}_{3}(\sqrt{-1})^{\times} \longrightarrow \mathbf{F}_{5}(\bar{\zeta})^{\times}
$$

\section{deternined by}

$$
\begin{aligned}
3 & \longmapsto 2 \\
\sqrt[4]{-1} & \longmapsto 1 \\
4 & \longmapsto \frac{1}{\zeta} \\
1+3 \sqrt{-1} & \longmapsto \bar{\zeta}
\end{aligned}
$$

(4) $\left.\bar{\rho}\right|_{G_{\mathbf{Q}_{3}(\sqrt{3})}}$ is given by the character

$$
\mathbf{Q}_{3}(\sqrt{3})^{\times} \longrightarrow \mathbf{F}_{5}(\bar{\zeta})^{\times}
$$

determined by

$$
\begin{aligned}
\sqrt{3} & \longmapsto \bar{\zeta}-\bar{\zeta}^{-1} \\
-1 & \longmapsto-1 \\
4 & \longmapsto 1 \\
1+\sqrt{3} & \longmapsto \bar{\zeta} .
\end{aligned}
$$

(5) $\left.\bar{\rho}\right|_{G_{\mathbf{Q}_{3}(\sqrt{-3})}}$ is given by the character

determined by

$$
\mathbf{Q}_{3}(\sqrt{-3})^{\times} \longrightarrow \mathbf{F}_{5}(\bar{\zeta})^{\times}
$$

$$
\begin{aligned}
\sqrt{-3} & \longmapsto \bar{\zeta}-\bar{\zeta}^{-1} \\
-1 & \longmapsto-1 \\
4 & \longmapsto 1 \\
1+3 \sqrt{-3} & \longmapsto \\
1+\sqrt{-3} & \longmapsto \bar{\zeta} .
\end{aligned}
$$

(6) $\left.\bar{\rho}\right|_{G_{\mathbf{Q}_{3}(\sqrt{-3})}}$ is given by the character

$$
\mathbf{Q}_{3}(\sqrt{-3})^{\times} \longrightarrow \mathbf{F}_{5}(\bar{\zeta})^{\times}
$$

determined by

$$
\begin{aligned}
\sqrt{-3} & \longmapsto \bar{\zeta}-\bar{\zeta}^{-1} \\
-1 & \longmapsto-1 \\
4 & \longmapsto \overline{1} \\
1+3 \sqrt{-3} & \longmapsto \bar{\zeta} \\
1+\sqrt{-3} & \longmapsto \bar{\zeta}^{i}
\end{aligned}
$$

where $i \in \mathbf{Z} / 3 \mathbf{Z}$.

To see that one of these cases can be attained, use the following facts, all of which are easy to verify.

- A subgroup of $\mathrm{GL}_{2}\left(\mathbf{F}_{5}\right)$ with a non-trivial normal subgroup of 3-power order is, up to conjugation, contained in the normaliser of $\mathbf{F}_{5}(\bar{\zeta})^{\times}$. 
- The intersection of $\mathrm{SL}_{2}\left(\mathbf{F}_{5}\right)$ with the normaliser of $\bar{\zeta}$ in $\mathrm{GL}_{2}\left(\mathbf{F}_{5}\right)$ is generated by $\bar{\zeta}$ and an element $\alpha$ such that $\alpha^{2}=-1$ and $\alpha \bar{\zeta} \alpha^{-1}=\bar{\zeta}^{-1}$.

- If $\beta \in \mathbf{F}_{5}(\bar{\zeta})^{\times}$, $\operatorname{det} \beta=3$, and $\alpha \beta \alpha^{-1}=-\beta$, then $\beta= \pm\left(\bar{\zeta}-\bar{\zeta}^{-1}\right)$.

In each case, we may choose an elliptic curve $E_{1 / \mathbf{Q}_{3}}$ such that the representation $\bar{\rho}_{E_{1}, 5}$ of $G_{3}$ on $E_{1}[5]\left(\overline{\mathbf{Q}}_{3}\right)$ is isomorphic to $\left.\bar{\rho}\right|_{G_{3}}$ and such that the representation $\bar{\rho}_{E_{1}, 3}$ of $G_{3}$ on $E_{1}[3]\left(\overline{\mathbf{Q}}_{3}\right)$ has the following form (where we use the same numbering as above).

(1) We place no restriction on $\bar{\rho}_{E_{1}, 3}$.

(2) The restriction of $\bar{\rho}_{E_{1}, 3}$ to $I_{3}$ has the form

$$
\left(\begin{array}{ll}
1 & * \\
0 & \omega
\end{array}\right)
$$

and is très ramifié. (Use Theorem 5.3.2 of $\mathrm{Man}$.)

(3) The restriction of $\bar{\rho}_{E_{1}, 3}$ to $I_{3}$ has the form

$$
\left(\begin{array}{ll}
1 & * \\
0 & \omega
\end{array}\right)
$$

and is très ramifié. (Use Theorem 5.3.2 of $[\mathrm{Man}]$.)

(4) The restriction of $\bar{\rho}_{E_{1}, 3}$ to $I_{3}$ has the form

$$
\left(\begin{array}{cc}
\omega & * \\
0 & 1
\end{array}\right)
$$

and is très ramifié. (Use $\S 5.4$ of Man.)

(5) The restriction of $\bar{\rho}_{E_{1}, 3}$ to $I_{3}$ has the form

$$
\left(\begin{array}{cc}
\omega & * \\
0 & 1
\end{array}\right)
$$

and is très ramifié. (Use $\S 5.4$ of Man].)

(6) $\bar{\rho}_{E_{1}, 3}$ has the form

$$
\left(\begin{array}{cc}
\omega & * \\
0 & 1
\end{array}\right)
$$

is très ramifié and remains indecomposable when restricted to the splitting field of $\bar{\rho}$. (Use Corollary 2.3 .2 below.)

In each case choose such an $E_{1}$ and fix an isomorphism $\alpha: \mathbf{F}_{5}^{2} \stackrel{\sim}{\rightarrow} E_{1}[5]\left(\overline{\mathbf{Q}}_{3}\right)$, such that the Weil pairing on $E_{1}[5]$ corresponds to the standard alternating pairing on $\mathbf{F}_{5}^{2}$, following the conventions in $\S 1$ of $[\mathrm{SBT}]$. The pair $\left(E_{1}, \alpha\right)$ defines a $\mathbf{Q}_{3}$-rational point on the smooth curve denoted $X_{\bar{\rho}}$ in [SBT]. We can find a 3 -adic open set $\mathcal{U} \subset X_{\bar{\rho}}\left(\mathbf{Q}_{3}\right)$ containing $\left(E_{1}, \alpha\right)$ such that if $\left(E_{2}, \beta\right)$ defines a point in $\mathcal{U}$, then $E_{2}[3] \cong E_{1}[3]$ as $\mathbf{F}_{3}\left[G_{3}\right]$-modules.

Using Ekedahl's version of the Hilbert Irreducibility Theorem (see Theorem 1.3 of [E]) and the argument of $\S 1$ of [SBT] we may find an elliptic curve $E_{/ \mathbf{Q}}$ and an $\mathbf{F}_{5}\left[G_{\mathbf{Q}}\right]$-module isomorphism $\beta$ of $\bar{\rho}$ with $E[5](\overline{\mathbf{Q}})$ such that (see also $\S 2$ of [Man])

- under $\beta$, the standard alternating pairing on $\mathbf{F}_{5}^{2}$ and the Weil pairing on $E[5]$ agree;

- the representation $\bar{\rho}_{E, 3}$ of $G_{\mathbf{Q}}$ on $E[3](\overline{\mathbf{Q}})$ is surjective onto $\operatorname{Aut}(E[3](\overline{\mathbf{Q}}))$;

- and $(E, \beta)$ defines a point of $\mathcal{U}$. 
Corresponding to the six types of $\bar{\rho}$ considered above, Proposition B.4.2 of [CDT ensures that the representation $\rho_{E, 3}$ of $G_{\mathbf{Q}}$ on the 3 -adic Tate module of $E$ is

(1) either, up to quadratic twist, ordinary in the sense of Wiles [Wi] or potentially Barsotti-Tate of some tamely ramified type;

(2) potentially Barsotti-Tate of type $\tau_{1}$;

(3) potentially Barsotti-Tate of type $\tau_{-1}$;

(4) potentially Barsotti-Tate of type $\tau_{3}$;

(5) potentially Barsotti-Tate of type $\tau_{-3}$;

(6) potentially Barsotti-Tate of extended type $\tau_{i}^{\prime}$.

In the first case, $E$ is modular by Theorem 7.2.1 of [CDT]. In the other cases we will simply write $\tau$ for the type/extended type. We see that $\bar{\rho}_{E, 3}\left(G_{3}\right)$ has centraliser $\mathbf{F}_{3}$ and the results of $\oint 2.1$ show that $\tau$ admits $\bar{\rho}_{E, 3}$ and that $\tau$ is weakly acceptable for $\bar{\rho}_{E, 3}$. Moreover $\left.\bar{\rho}_{E, 3}\right|_{\mathrm{Gal}(\overline{\mathbf{Q}} / \mathbf{Q}(\sqrt{-3}))}$ is absolutely irreducible and, by the LanglandsTunnell theorem (see [Wi]), modular. Thus by Theorems 1.4.1 and 1.4.2 we see that $\rho_{E, 3}$ is modular. We deduce that $E$ is modular, so $\bar{\rho} \cong \bar{\rho}_{E, 5}$ is modular.

Combining this theorem with Theorem 7.2.4 of [CDT] we immediately obtain the following corollary.

Theorem 2.2.2. Every elliptic curve defined over the rational numbers is modular.

2.3. An extension of a result of Manoharmayum. The following facts follow at once from Man, particularly the classification given just before Theorem 5.4 .2 of that paper. Consider elliptic curves $E$ over $\mathbf{Q}_{3}$ with minimal Weierstrass equation $Y^{2}=X^{3}+A X+B$, where

$$
A \equiv B+3 \equiv 0 \bmod 9,
$$

so $\bar{\rho}_{E, 3}$ has the form $\left(\begin{array}{cc}\omega & * \\ 0 & 1\end{array}\right)$ and is très ramifié. This leaves three possibilities for the equivalence class of $\bar{\rho}_{E, 3}$. Fix $\bar{\zeta}$ in $\operatorname{GL}_{2}\left(\mathbf{F}_{5}\right)$ with $\bar{\zeta}^{3}=1$ but $\bar{\zeta} \neq 1$. The action of $G_{\mathbf{Q}_{3}(\sqrt{-3})}$ on $E[5]\left(\overline{\mathbf{Q}}_{3}\right)$ is via a representation of the form

$$
\begin{aligned}
\sqrt{-3} & \longmapsto \delta\left(\bar{\zeta}-\bar{\zeta}^{-1}\right) \\
-1 & \longmapsto-1 \\
4 & \longmapsto 1 \\
1+\sqrt{-3} & \longmapsto \bar{\zeta}^{i} \\
1+3 \sqrt{-3} & \longmapsto \bar{\zeta}
\end{aligned}
$$

for some $\delta= \pm 1$ and some $i \in \mathbf{Z} / 3 \mathbf{Z}$. All nine possibilities for the pair $\left(\bar{\rho}_{E, 3}, i\right)$ satisfying these conditions can arise for some such choice of $A$ and $B$.

Lemma 2.3.1. With the above notation and assumptions, we have $\delta=1$.

Proof. Let $F=\mathbf{Q}_{3}(\sqrt{-3}, \beta, \alpha)$, where $\beta^{2}=-\sqrt{-3}$ and

$$
\alpha^{3}+A \alpha+B=9 \sqrt{-3} .
$$

$F$ is a totally ramified abelian extension of $\mathbf{Q}_{3}(\sqrt{-3})$ of degree 6 , with uniformiser $\varpi=\alpha / \beta$. The change of coordinates $Y \mapsto \varpi^{15} Y, X \mapsto \varpi^{10} X+\alpha$ shows that $E$ has good reduction over $F$, and the reduction is isomorphic to

$$
Y^{2}=X^{3}-X-1
$$


The arithmetic Frobenius of $W_{F}$ therefore has trace 3 on $E[5]$. Since

$$
N_{F / \mathbf{Q}_{3}(\sqrt{-3})}(\varpi) \equiv \sqrt{-3}(1-3 \sqrt{-3}) \bmod 9 \sqrt{-3},
$$

we conclude that

$$
\operatorname{tr} \delta\left(\bar{\zeta}-\bar{\zeta}^{-1}\right) \bar{\zeta}^{-1}=3
$$

so $\delta=1$.

Twisting by quadratic characters we immediately deduce the following corollary.

Corollary 2.3.2. Let $\bar{\rho}_{3}: G_{3} \rightarrow \mathrm{GL}_{3}\left(\mathbf{F}_{3}\right)$ have the form

$$
\left(\begin{array}{cc}
\omega & * \\
0 & 1
\end{array}\right) \text { or }\left(\begin{array}{cc}
1 & * \\
0 & \omega
\end{array}\right)
$$

and be très ramifié. Let $\bar{\rho}_{5}: G_{3} \rightarrow \mathrm{GL}_{2}\left(\mathbf{F}_{5}\right)$ have cyclotomic determinant and restriction to $G_{\mathbf{Q}_{3}(\sqrt{-3})}$ given by a character

$$
\mathbf{Q}_{3}(\sqrt{-3})^{\times} \longrightarrow \mathbf{F}_{5}(\bar{\zeta})^{\times}
$$

determined by

$$
\begin{aligned}
\sqrt{-3} & \longmapsto\left(\bar{\zeta}-\bar{\zeta}^{-1}\right) \\
-1 & \longmapsto-1 \\
4 & \longmapsto 1 \\
1+\sqrt{-3} & \longmapsto \bar{\zeta}^{i} \\
1+3 \sqrt{-3} & \longmapsto \bar{\zeta}
\end{aligned}
$$

for some $i \in \mathbf{Z} / 3 \mathbf{Z}$. There is an elliptic curve $E_{/ \mathbf{Q}_{3}}$, with $E[3]\left(\overline{\mathbf{Q}}_{3}\right) \sim \bar{\rho}_{3}$ and $E[5]\left(\overline{\mathbf{Q}}_{3}\right) \sim \bar{\rho}_{5}$. In particular, the action of $I_{3}$ on $T_{5} E$ factors through a finite group and so $E$ has potentially good reduction.

\section{Admittance}

In this section we will check Lemmas 2.1.1, 2.1.3 and 2.1.5. We freely use the terminology introduced in $\$ 1.2$ and $\$ 1.3$.

3.1. The case of $\tau_{1}$. In this case $\sigma_{\tau_{1}}$ is the induction from $U_{0}(9)$ to $\mathrm{GL}_{2}\left(\mathbf{Z}_{3}\right)$ of a character of order 3 . Its reduction modulo a prime above 3 has the same

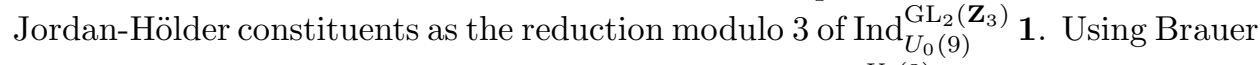
characters, we see that the reduction modulo 3 of $\operatorname{Ind}_{U_{0}(9)}^{U_{0}(3)} \mathbf{1}$ has Jordan-Hölder constituents $\sigma_{0,0}^{\prime}, \sigma_{0,0}^{\prime}$ and $\sigma_{1,1}^{\prime}$. Thus, $\tau_{1}$ admits $\sigma_{0,0}, \sigma_{2,0}, \sigma_{0,1}$ and $\sigma_{2,1}$, the latter two simply. Lemma 2.1 .1 follows in this case.

3.2. The case of $\tau_{-1}$. Let $U$ denote the subgroup of $\mathrm{GL}_{2}\left(\mathbf{Z}_{3}\right)$ consisting of matrices

$$
\left(\begin{array}{ll}
a & b \\
c & d
\end{array}\right)
$$

with $a \equiv d \bmod 3$ and $b+c \equiv 0 \bmod 3$, so $\sigma_{\tau_{-1}}$ is the induction from $U$ to $\mathrm{GL}_{2}\left(\mathbf{Z}_{3}\right)$ of a character of order 3 . Upon reduction modulo a prime above 3 this will have the same Jordan-Hölder constituents as the reduction modulo $3{\text { of } \operatorname{Ind}_{U} \mathrm{GL}_{2}\left(\mathbf{Z}_{3}\right)} \mathbf{1}$. If $\psi$ denotes the non-trivial character of $\mathbf{F}_{3}^{\times}$and $\phi$ a character of $\mathbf{F}_{9}^{\times}$of order 4 , then this latter induction splits up as the sum of the representations of $\mathrm{GL}_{2}\left(\mathbf{Z}_{3}\right) \rightarrow \mathrm{GL}_{2}\left(\mathbf{F}_{3}\right)$ denoted $1, \mathrm{sp}_{\psi}$ and $\Theta(\phi)$ in $\S 3.1$ of [CDT]. By Lemma 3.1.1 of [CDT] we see that 
$\tau_{-1}$ admits $\sigma_{0,0}, \sigma_{2,1}$ and $\sigma_{0,1}$, the latter two simply. Lemma 2.1.1 follows in this case.

3.3. The case of $\tau_{ \pm 3}$. Let $U$ denote the subgroup of $\mathrm{GL}_{2}\left(\mathbf{Z}_{3}\right)$ consisting of matrices

$$
\left(\begin{array}{ll}
a & b \\
c & d
\end{array}\right)
$$

with $a \equiv d \bmod 3$ and $c \equiv 0 \bmod 3$. Then $\sigma_{\tau_{ \pm 3}}$ is the induction from $U$ to $U_{0}(3)$ of a character of order 3 . Upon reduction modulo a prime above 3 this will have the same Jordan-Hölder constituents as the reduction modulo 3 of $\operatorname{Ind}_{U}^{U_{0}(3)} \mathbf{1}$. Thus, $\tau_{ \pm 3}$ simply admits $\sigma_{0,0}^{\prime}$ and $\sigma_{1,1}^{\prime}$. Lemma 2.1 .3 follows.

3.4. The case of $\tau_{i}^{\prime}$. Let $\chi$ be the character of $\mathbf{Q}_{3}(\sqrt{-3})^{\times}$as in (2.1.1). Let $\psi$ be a character of $\mathbf{Q}_{3}$ with kernel $\mathbf{Z}_{3}$ and which sends $1 / 3$ to $\zeta$. If $x \in(3 \sqrt{-3}) \mathbf{Z}_{3}[\sqrt{-3}]$ we have

$$
\chi(1+x)=\psi\left(\operatorname{tr}_{\mathbf{Q}_{3}(\sqrt{-3}) / \mathbf{Q}_{3}}(-x \sqrt{-3} / 54)\right) .
$$

We deduce that if $\chi^{\prime \prime}$ is the character used to define $\sigma_{\tau_{i}^{\prime}}$ in $\$ 1.2$, then $\chi^{\prime \prime}(\sqrt{-3})=$ $\left(\zeta-\zeta^{-1}\right)^{-1}$.

Let $U$ denote the subgroup of $\mathrm{GL}_{2}\left(\mathbf{Z}_{3}\right)$ consisting of matrices

$$
\left(\begin{array}{cc}
a & b \\
3 c & d
\end{array}\right)
$$

with $a \equiv d \bmod 3$ and $b+c \equiv 0 \bmod 3$. Let $\widetilde{U}$ be the group generated by $w_{3}$ (see (1.2.1) ) and $U$, so $\sigma_{\tau_{i}^{\prime}}$ is the representation of $\widetilde{U}_{0}(3)$ induced from a character of $\widetilde{U}$ which sends $w_{3}$ to 1 and has order 3 when restricted to $U$. Thus, the Jordan-Hölder constituents of the reduction of $\sigma_{\tau_{i}^{\prime}}$ modulo a prime above 3 are the same as the

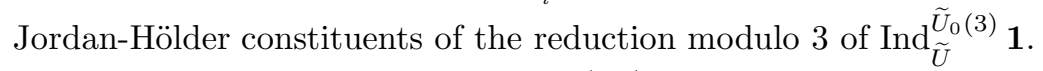

Let $V$ denote the subgroup of $\mathrm{GL}_{2}\left(\mathbf{Z}_{3}\right)$ consisting of matrices

$$
\left(\begin{array}{cc}
a & b \\
3 c & d
\end{array}\right)
$$

with $a \equiv d \bmod 3$. Let $\widetilde{V}$ be the group generated by $w_{3}$ and $V$, and let $\nu$ denote the character of $\widetilde{V} / V$ which sends $w_{3}$ to -1 . We have

$$
\operatorname{Ind}_{\widetilde{U}}^{\widetilde{V}}(\mathbf{1}) \sim \mathbf{1} \oplus \operatorname{Ind}_{V \mathbf{Q}_{3}^{\times}}^{\widetilde{V}} \eta
$$

where $\eta$ is a non-trivial character of $V / U=\left(V \mathbf{Q}_{3}^{\times}\right) /\left(U \mathbf{Q}_{3}^{\times}\right)$. The reduction modulo a prime above 3 of this (3-dimensional) representation has the same Jordan-Hölder constituents as the reduction modulo 3 of $\mathbf{1} \oplus \mathbf{1} \oplus \nu$. Thus, $\tau_{i}^{\prime}$ admits $\sigma_{\{0\}, 1}^{\prime}, \sigma_{\{1\}, 1}^{\prime}$, $\sigma_{\{0\},-1}^{\prime}$ and $\sigma_{\{1\},-1}^{\prime}$, the latter two simply. Lemma 2.1.5 follows.

\section{NeW DEFormation PROBlems}

In this section we begin the proof of Theorems 2.1.2 2.1.4 and 2.1.6. One could approach this directly by using the results of [Br2] to attempt to describe $R_{V, \mathcal{O}}^{D}$ (resp. $R_{V, \mathcal{O}}^{D^{\prime}}$ ). At least one of the authors of this paper (Taylor) thinks that such an approach holds out more promise of attacking the non-acceptable case, and another author (Breuil) has indeed made several computations along these lines. However in the present case it seems to be easier to proceed less directly. 
To this end we will use ad hoc arguments to define deformation problems, which will be represented by $\mathcal{O}$-algebras $S$ such that

- $\operatorname{dim}_{k} \mathfrak{m}_{S} /\left(\wp_{K}, \mathfrak{m}_{S}^{2}\right) \leq 1$,

- and the map $R_{V, \mathcal{O}} \rightarrow R_{V, \mathcal{O}}^{D}$ (resp. $\left.R_{V, \mathcal{O}}^{D^{\prime}}\right)$ factors through $S$.

An important advantage of this approach is that to calculate $\mathfrak{m}_{S} /\left(\wp_{K}, \mathfrak{m}_{S}^{2}\right)$ one need only work in the category of finite flat group schemes killed by a prime. Breuil modules (see 95) for finite flat group schemes killed by an odd prime are significantly simpler than the general case (of prime power torsion). This is particularly true when we also use descent data. On the other hand, to suitably define the new deformation problems is rather delicate. That is what we will do in this section.

4.1. Some generalities on group schemes. In this section, and in 4.2 again be an arbitrary rational prime. Moreover $R$ will denote a complete discrete valuation ring with fraction field $F^{\prime}$ of characteristic zero and perfect residue field $k$ of characteristic $\ell$. We will let $\Gamma$ denote a finite group of continuous automorphisms of $R$ and we will let $F_{0}$ denote the subfield of $F^{\prime}$ consisting of elements fixed by $\Gamma$. Thus $F^{\prime} / F_{0}$ will be finite and Galois with group $\Gamma$. In our applications of these results it suffices to consider the case where $F_{0}$ is a finite extension of $\mathbf{Q}_{3}$ (although we will occasionally pass to the completion of the maximal unramified extension of $F)$.

Lemma 4.1.1. Let $\mathcal{G}$ be a finite flat $R$-group scheme. Scheme theoretic closure gives a bijection between subgroup schemes of $\mathcal{G} \times F^{\prime}$ and finite flat closed subgroup schemes of $\mathcal{G}$.

(See for instance $\S 1.1$ of $\mathrm{Co}$.)

Lemma 4.1.2. Let $\mathcal{G}_{1}$ and $\mathcal{G}_{2}$ be finite flat group schemes over $R$ which have locallocal closed fibre. Suppose that $\mathcal{G}_{1}$ and $\mathcal{G}_{2}$ are the only finite flat $R$-group schemes with local-local closed fibre which have generic fibres $\mathcal{G}_{1} \times F^{\prime}$ and $\mathcal{G}_{2} \times F^{\prime}$ respectively. Suppose also that we have an exact sequence of finite flat $R$-group schemes

$$
(0) \longrightarrow \mathcal{G}_{1} \longrightarrow \mathcal{G} \longrightarrow \mathcal{G}_{2} \longrightarrow(0) \text {. }
$$

Then $\mathcal{G}$ is the unique finite flat $R$-group scheme with local-local closed fibre and with generic fibre $\mathcal{G} \times F$.

Proof. Let $\mathcal{G}_{+}$and $\mathcal{G}_{-}$denote the maximal and minimal local-local models for $\mathcal{G} \times F$. The proof that these exist follows the proof of Proposition 2.2.2 of [Ra] and uses the fact that the Cartier dual of a local-local finite flat group scheme is local-local. We must show that the canonical map $\mathcal{G}_{+} \rightarrow \mathcal{G}_{-}$is an isomorphism. The schemetheoretic closure of $\mathcal{G}_{1} \times F$ in $\mathcal{G}_{ \pm}$must be isomorphic to $\mathcal{G}_{1}$ (by uniqueness), so we have closed immersions $\mathcal{G}_{1} \hookrightarrow \mathcal{G}_{ \pm}$extending $\mathcal{G}_{1} \times F \hookrightarrow \mathcal{G}_{ \pm} \times F$. Similarly $\mathcal{G}_{ \pm} / \mathcal{G}_{1}$ must be isomorphic to $\mathcal{G}_{2}$. This gives a commutative diagram with exact rows:

$$
\begin{aligned}
& 0 \rightarrow \mathcal{G}_{1} \rightarrow \mathcal{G}_{+} \rightarrow \mathcal{G}_{2} \rightarrow 0 \\
& 0 \rightarrow \begin{array}{l}
\downarrow \\
\mathcal{G}_{1}
\end{array} \rightarrow \mathcal{G}_{-} \rightarrow \mathcal{G}_{2} \rightarrow 0
\end{aligned}
$$

The vertical maps $\mathcal{G}_{1} \rightarrow \mathcal{G}_{1}$ and $\mathcal{G}_{2} \rightarrow \mathcal{G}_{2}$ induce isomorphisms on the generic fibre and hence are isomorphisms. This is because some power of them is the identity on the generic fibre and hence is the identity. Working in the abelian category of fppf abelian sheaves over $\operatorname{Spec} R$, the middle map must also be an isomorphism. 
When $\mathcal{G}$ has $\ell$-power order, we will let $D(\mathcal{G})$ denote the classical (contravariant) Dieudonné module of $\mathcal{G} \times k$. It is a $W(k)$-module equipped with a Frobenius operator $\mathbf{F}$ and a Verschiebung operator $\mathbf{V}$. We have $\mathbf{F V}=\mathbf{V F}=\ell$ and for all $x \in W(k)$, $\mathbf{F} x=\left(\right.$ Frob $\left._{\ell} x\right) \mathbf{F}$ and $\mathbf{V} x=\left(\operatorname{Frob}_{\ell}^{-1} x\right) \mathbf{V}$.

If $\mathcal{G}$ is a finite flat $R$-group scheme, then by descent data for $\mathcal{G}$ over $F_{0}$ we mean a collection $\{[g]\}$ of group scheme isomorphisms over $R$

$$
[g]: \mathcal{G} \stackrel{\sim}{\longrightarrow} g \mathcal{G}
$$

for $g \in \Gamma$ such that for all $g, h \in \Gamma$ we have

$$
[g h]=\left({ }^{g}[h]\right) \circ[g] .
$$

Note that this is not descent data in the sense of Grothendieck, since $R / R^{\Gamma}$ might be ramified. However, $\operatorname{Spec} F^{\prime} / \operatorname{Spec} F_{0}$ is étale, so by étale descent we obtain a finite flat group scheme $(\mathcal{G},\{[g]\})_{F_{0}}$ over $F_{0}$ together with an isomorphism

$$
(\mathcal{G},\{[g]\})_{F_{0}} \times{ }_{F_{0}} F^{\prime} \cong \mathcal{G} \times{ }_{R} F^{\prime}
$$

compatible with descent data. We also obtain a natural left action of $\Gamma$ on the Dieudonné module $D(\mathcal{G})$, semi-linear with respect to the $W(k)$-module structure and commuting with $\mathbf{F}$ and $\mathbf{V}$. We refer to the pair $(\mathcal{G},\{[g]\})$ as an $R$-group scheme with descent data relative to $F_{0}$. One defines morphisms of such objects to be morphisms of $R$-group schemes which commute with the descent data. By a closed finite flat subgroup scheme with descent data we mean a closed finite flat subgroup scheme such that the descent data on the ambient scheme takes the subscheme to itself. Quotients by such subobjects are defined in the obvious way. Thus we obtain an additive category with a notion of short exact sequence. Suppose that $G$ is a finite flat $F_{0}$-group scheme. By a model with descent data (or simply model) for $G$ over $R$ we shall mean a triple $(\mathcal{G},\{[g]\}, i)$, where $(\mathcal{G},\{[g]\})$ is an $R$-group scheme with descent data relative to $F_{0}$ and where $i:(\mathcal{G},\{[g]\})_{F_{0}} \stackrel{\sim}{\rightarrow} G$. Sometimes we will suppress $i$ from the notation. It is easy to check that isomorphism classes of models admitting descent data for $G$ over $R$ form a sublattice of the lattice of models for $G_{/ F^{\prime}}$ over $R$. The following lemma follows without difficulty from Lemma 4.1.1

Lemma 4.1.3. Let $F^{\prime} / F_{0}$ be a finite Galois extension as above, and let $(\mathcal{G},\{[g]\})$ be a finite flat $R$-group scheme with descent data relative to $F_{0}$. Base change from $F_{0}$ to $F^{\prime}$, followed by scheme theoretic closure, gives a bijection between subgroup schemes of $(\mathcal{G},\{[g]\})_{F_{0}}$ and closed finite flat subgroup schemes with descent data in $(\mathcal{G},\{[g]\})$.

We let $\mathcal{F F}_{F^{\prime}}$ denote the category of finite flat group schemes over $R$ and $\mathcal{F D}_{F^{\prime}} / F_{0}$ the category of finite flat group schemes over $R$ with descent data over $F_{0}$. Let $W(k)[\mathbf{F}, \mathbf{V}][\Gamma]$ denote the (non-commutative) $W(k)$-algebra generated by elements $\mathbf{F}, \mathbf{V}$ and $[g]$ for $g \in \Gamma$ satisfying

- $[g h]=[g][h]$ for all $g, h \in \Gamma$;

- $[g] \mathbf{F}=\mathbf{F}[g]$ and $[g] \mathbf{V}=\mathbf{V}[g]$ for all $g \in \Gamma$;

- $\mathbf{F V}=\mathbf{V F}=\ell$

- $[g] x=(g x)[g]$ for all $x \in W(k)$ and $g \in \Gamma$;

- $\mathbf{F} x=\left(\right.$ Frob $\left._{\ell} x\right) F$ and $\mathbf{V} x=\left(\operatorname{Frob}_{\ell}^{-1} x\right) \mathbf{V}$ for all $x \in W(k)$.

If $\mathcal{J}$ is a two-sided ideal in $W(k)[\mathbf{F}, \mathbf{V}][\Gamma]$, we will let $\mathcal{F D}_{F^{\prime} / F_{0}, \mathcal{J}}$ denote the full subcategory of $\mathcal{F D}_{F^{\prime}} / F_{0}$ consisting of objects $(\mathcal{G},\{[g]\})$ such that $\mathcal{J}$ annihilates $D(\mathcal{G})$. If $(\mathcal{G},\{[g]\})$ is an object of $\mathcal{F D}_{F^{\prime} / F_{0}, \mathcal{J}}$ and if $(\mathcal{H},\{[g]\}) \subset(\mathcal{G},\{[g]\})$ is a closed finite 
flat subgroup scheme with descent data, then $(\mathcal{H},\{[g]\})$ and $(\mathcal{G},\{[g]\}) /(\mathcal{H},\{[g]\})$ are again objects of $\mathcal{F} \mathcal{D}_{F^{\prime} / F_{0}, \mathcal{J}}$.

Lemma 4.1.4. For $\mathcal{J}$ a two-sided ideal of the ring $W(k)[\mathbf{F}, \mathbf{V}][\Gamma]$, choose objects $\left(\mathcal{G}_{1},\{[g]\}\right)$ and $\left(\mathcal{G}_{2},\{[g]\}\right)$ in $\mathcal{F D}_{F^{\prime} / F_{0}, \mathcal{J}}$ so that $\left(\mathcal{G}_{1},\{[g]\}\right)_{F_{0}} \cong\left(\mathcal{G}_{2},\{[g]\}\right)_{F_{0}}$. Let $G$ denote the base change of this $F_{0}$-group scheme to $F^{\prime}$, so $G$ has canonical descent data relative to $F^{\prime} / F_{0}$. Then the sup and inf of $\mathcal{G}_{1}$ and $\mathcal{G}_{2}$ in the lattice of integral models for $G$ are stable under the descent data on $G$ and with this descent data are objects of $\mathcal{F D}_{F^{\prime} / F_{0}, \mathcal{J}}$.

Proof. By uniqueness of the inf and sup, they are stable under the descent data on the generic fibre. It follows from Raynaud's construction of the inf and sup (Proposition 2.2.2 of [Ra] in terms of subgroup schemes and quotients of $\mathcal{G}_{1} \times \mathcal{G}_{2}$ that the sup and inf are objects of $\mathcal{F D}_{F^{\prime} / F_{0}, \mathfrak{J}}$.

Corollary 4.1.5. Let J be a two-sided ideal of the ring $W(k)[\mathbf{F}, \mathbf{V}][\Gamma]$. Let

$$
(0) \longrightarrow G_{1} \longrightarrow G \longrightarrow G_{2} \longrightarrow(0)
$$

be an exact sequence of finite flat group schemes over $F_{0}$. Let $\left(\mathcal{G}_{1},\{[g]\}\right)$ and $\left(\mathcal{G}_{2},\{[g]\}\right)$ be objects of $\mathcal{F} \mathcal{D}_{F^{\prime} / F_{0}, \mathcal{J}}$ such that $\left(\mathcal{G}_{1},\{[g]\}\right)_{F_{0}} \cong G_{1}$ and $\left(\mathcal{G}_{2},\{[g]\}\right)_{F_{0}} \cong$ $G_{2}$. Suppose that for all objects $(\mathcal{G},\{[g]\})$ of $\mathcal{F D}_{F^{\prime} / F_{0}, \mathcal{J}}$ with $(\mathcal{G},\{[g]\})_{F_{0}} \cong G$, the filtration on $(\mathcal{G},\{[g]\})$ induced by the filtration on $G$ has subobject isomorphic to $\left(\mathcal{G}_{1},\{[g]\}\right)$ and quotient isomorphic to $\left(\mathcal{G}_{2},\{[g]\}\right)$ (without any assumed compatibility with (4.1.1D). Then there is at most one model for $G$ in $\mathcal{F D}_{F^{\prime} / F_{0}, J}$.

Proof. By Lemma 4.1.4, it suffices to prove that if $\left(\mathcal{G}_{+},\{[g]\}, i_{+}\right)$and $\left(\mathcal{G}_{-},\{[g]\}, i_{-}\right)$ are two such models with a morphism between them, then the morphism between them must be an isomorphism. In such a case we have a commutative diagram with exact rows:

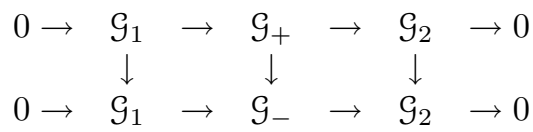

The vertical maps $\mathcal{G}_{1} \rightarrow \mathcal{G}_{1}$ and $\mathcal{G}_{2} \rightarrow \mathcal{G}_{2}$ induce isomorphisms on the generic fibre and hence are isomorphisms. This is because some power of them is the identity on the generic fibre and hence is the identity. Working in the abelian category of fppf abelian sheaves over $\operatorname{Spec} R$, the middle map must also be an isomorphism.

4.2. Filtrations. We keep the notation and assumptions of the previous section. Let $\Sigma$ be a finite non-empty set of objects $\left(\mathcal{G}_{i},\{[g]\}\right)$ of $\mathcal{F D}_{F^{\prime} / F_{0},(\mathcal{J}, \ell)}$. (Note the $\ell$ in the subscript $(\mathcal{J}, \ell)$, which denotes the two-sided ideal generated by $\mathcal{J}$ and $\ell$.) Suppose that

$$
\begin{aligned}
\operatorname{Hom}\left(\left(\mathcal{G}_{i},\{[g]\}\right),\left(\mathcal{G}_{j},\{[g]\}\right)\right) & =\operatorname{Hom}\left(\left(\mathcal{G}_{i},\{[g]\}\right)_{F_{0}},\left(\mathcal{G}_{j},\{[g]\}\right)_{F_{0}}\right) \\
& = \begin{cases}0 & \text { if } i \neq j, \\
\text { finite field } & \text { if } i=j\end{cases}
\end{aligned}
$$

(in particular, the objects in $\Sigma$ are non-zero and pairwise non-isomorphic). By a $\Sigma$ filtration on a finite flat $F_{0}$-group scheme $G$ we mean an increasing filtration $\mathrm{Fil}^{j} G$ such that for all $j$ the graded piece $\mathrm{Fil}^{j} G / \mathrm{Fil}^{j-1} G$ is isomorphic to $\left(\mathcal{G}_{i(j)},\{[g]\}\right)_{F_{0}}$ for a (unique) $\left(\mathcal{G}_{i(j)},\{[g]\}\right) \in \Sigma$. The following lemma is proved by the standard Jordan-Hölder argument. 
Lemma 4.2.1. If $G$ is a finite flat $F_{0}$-group scheme which admits a $\Sigma$-filtration and if $H$ is a quotient or subobject of $G$ which admits a $\Sigma$-filtration, then any $\Sigma$-filtration of $H$ can be extended to a $\Sigma$-filtration of $G$. In addition, all $\Sigma$-filtrations of $G$ have the same length and the same set of successive quotients (with multiplicities).

We say that an object $(\mathcal{G},\{[g]\})$ of $\mathcal{F D}_{F^{\prime} / F_{0}, \mathfrak{J}}$ is weakly filtered by $\Sigma$ if there is some increasing filtration $\operatorname{Fil}^{j}(\mathcal{G},\{[g]\})$ of $(\mathcal{G},\{[g]\})$ by closed subobjects such that for all $j$, the graded piece

$$
\operatorname{Fil}^{j}(\mathcal{G},\{[g]\}) / \mathrm{Fil}^{j-1}(\mathcal{G},\{[g]\})
$$

is isomorphic to an element of $\Sigma$. We say that an object $(\mathcal{G},\{[g]\})$ of $\mathcal{F} \mathcal{D}_{F^{\prime} / F_{0}, \mathcal{J}}$ is strongly filtered by $\Sigma$ if $(\mathcal{G},\{[g]\})$ is weakly filtered by $\Sigma$ and if for every $\Sigma$-filtration of $(\mathcal{G},\{[g]\})_{F_{0}}$ the corresponding filtration of $(\mathcal{G},\{[g]\})$ satisfies

$$
\operatorname{Fil}^{j}(\mathcal{G},\{[g]\}) / \mathrm{Fil}^{j-1}(\mathcal{G},\{[g]\})
$$

is isomorphic to an element of $\Sigma$ for all $j$. The following lemma follows at once from the definitions and from Lemma 4.2.1

Lemma 4.2.2. (1) If $(\mathcal{G},\{[g]\})$ and $\left(\mathcal{G}^{\prime},\{[g]\}\right)$ are objects of $\mathcal{F} \mathcal{D}_{F^{\prime} / F_{0}, \mathcal{J}}$ which are weakly filtered by $\Sigma$, then $(\mathcal{G},\{[g]\}) \times\left(\mathcal{G}^{\prime},\{[g]\}\right)$ is also weakly filtered by $\Sigma$.

(2) Let $(\mathcal{G},\{[g]\})$ and $\left(\mathcal{G}^{\prime},\{[g]\}\right)$ be objects of $\mathcal{F D}_{F^{\prime} / F_{0}, \mathcal{J}}$ with $\left(\mathcal{G}^{\prime},\{[g]\}\right)$ a closed subobject or quotient of $(\mathcal{G},\{[g]\})$. Suppose that $(\mathcal{G},\{[g]\})$ is strongly filtered by $\Sigma$ and that $\left(\mathcal{G}^{\prime},\{[g]\}\right)_{F_{0}}$ admits a $\Sigma$-filtration. Then $\left(\mathcal{G}^{\prime},\{[g]\}\right)$ is strongly filtered by $\Sigma$.

If any object of $\mathcal{F} \mathcal{D}_{F^{\prime} / F_{0}, \mathcal{J}}$ which is weakly filtered by $\Sigma$ is strongly filtered by $\Sigma$, then we will let $\mathcal{F} \mathcal{D}_{F^{\prime} / F_{0}, \mathcal{J}, \Sigma}$ denote the full subcategory of $\mathcal{F} \mathcal{D}_{F^{\prime} / F_{0}}$ J J consisting of objects which are weakly (and therefore strongly) filtered by $\Sigma$.

Lemma 4.2.3. Suppose that any object of $\mathcal{F D}_{F^{\prime} / F_{0}, \mathcal{J}}$ which is weakly filtered by $\Sigma$ is strongly filtered by $\Sigma$. Let $G$ be a finite flat $F_{0}$-group scheme. If $\left(\mathcal{G}_{1},\{[g]\}\right)$ and $\left(\mathcal{G}_{2},\{[g]\}\right)$ are two objects of $\mathcal{F D}_{F^{\prime} / F_{0}, \mathfrak{J}, \Sigma}$ with isomorphisms

$$
i_{j}: G \stackrel{\sim}{\longrightarrow}\left(\mathcal{G}_{j},\{[g]\}\right)_{F_{0}}
$$

for $j=1,2$, then there is a unique isomorphism

$$
\phi:\left(\mathcal{G}_{1},\{[g]\}\right) \stackrel{\sim}{\longrightarrow}\left(\mathcal{G}_{2},\{[g]\}\right)
$$

such that on the generic fibre $i_{2}=\phi \circ i_{1}$.

Proof. It follows from Raynaud's construction of sup and inf that the sup and inf of $\left(\left(\mathcal{G}_{1},\{[g]\}\right), i_{1}\right)$ and $\left(\left(\mathcal{G}_{2},\{[g]\}\right), i_{2}\right)$ are again objects of $\mathcal{F D}_{F^{\prime} / F_{0}, \mathcal{J}, \Sigma}$. Thus we may suppose that there exists a map $\phi:\left(\mathcal{G}_{1},\{[g]\}\right) \rightarrow\left(\mathcal{G}_{2},\{[g]\}\right)$ such that on the generic fibre $i_{2}=\phi \circ i_{1}$. We will argue by induction on the rank of $G$ that $\phi$ is an isomorphism.

If $\left(\mathcal{G}_{1},\{[g]\}\right)$ is isomorphic to an element of $\Sigma$, then the result follows by our assumption on $\Sigma$.

If $\left(\mathcal{G}_{1},\{[g]\}\right)$ is not isomorphic to an element of $\Sigma$, then choose an exact sequence

$$
(0) \longrightarrow\left(\mathcal{G}_{11},\{g\}\right) \longrightarrow\left(\mathcal{G}_{1},\{g\}\right) \longrightarrow\left(\mathcal{G}_{12},\{g\}\right) \longrightarrow(0),
$$

where $\left(\mathcal{G}_{11},\{g\}\right)$ and $\left(\mathcal{G}_{12},\{g\}\right)$ are weakly filtered by $\Sigma$. Let $\left(\mathcal{G}_{21},\{[g]\}\right)$ denote the closed subobject of $\left(\mathcal{G}_{2},\{[g]\}\right)$ corresponding to $\left(\mathcal{G}_{11},\{[g]\}\right)_{F_{0}}$ and define 
$\left(\mathcal{G}_{22},\{[g]\}\right)=\left(\mathcal{G}_{2},\{[g]\}\right) /\left(\mathcal{G}_{21},\{[g]\}\right)$. Then we have a commutative diagram with exact rows

$$
\begin{aligned}
& 0 \rightarrow \mathcal{G}_{11} \rightarrow \mathcal{G}_{1} \rightarrow \mathcal{G}_{12} \rightarrow 0 \\
& 0 \rightarrow \begin{array}{c}
\downarrow \\
\mathcal{G}_{21}
\end{array} \rightarrow \begin{array}{l}
\downarrow \\
\mathcal{G}_{2}
\end{array} \rightarrow \begin{array}{l}
\downarrow \\
\mathcal{G}_{22}
\end{array} \rightarrow 0
\end{aligned}
$$

compatible with descent data, where the central vertical arrow is $\phi$ and where by inductive hypothesis the outside vertical arrows are isomorphisms. Working in the abelian category of fppf abelian sheaves over $\operatorname{Spec} R$, we see that $\phi$ is an isomorphism.

The following lemma and its corollary give criteria for the equivalence of the notions of being weakly filtered by $\Sigma$ and of being strongly filtered by $\Sigma$.

Lemma 4.2.4. Fix $\mathcal{J}$ and $\Sigma$ as above. Suppose that for any pair of (possibly equal) elements $\left(\mathcal{G}^{\prime},\{[g]\}\right)$ and $\left(\mathcal{G}^{\prime \prime},\{[g]\}\right)$ in $\Sigma$, the natural map

$$
\operatorname{Ext}_{\mathcal{F}_{F_{F^{\prime} / F_{0},(J, \ell)}}^{1}}^{1}\left(\left(\mathcal{G}^{\prime \prime},\{[g]\}\right),\left(\mathcal{G}^{\prime},\{[g]\}\right)\right) \longrightarrow \operatorname{Ext}_{\mathbf{F}_{\ell}\left[G_{F_{0}}\right]}^{1}\left(\left(\mathcal{G}^{\prime \prime},\{[g]\}\right)_{F_{0}},\left(\mathcal{G}^{\prime},\{[g]\}\right)_{F_{0}}\right)
$$

is injective. Then any object $(\mathcal{G},\{[g]\})$ of $\mathcal{F D}_{F^{\prime} / F_{0}, \mathcal{J}}$ which is weakly filtered by $\Sigma$ is also strongly filtered by $\Sigma$.

Proof. For brevity, we say "weakly/strongly filtered" rather than "weakly/strongly filtered by $\Sigma$ " since the data $\Sigma$ is fixed for the entire proof. Also, we omit the specification of descent data from the notation, but it should not be forgotten.

Suppose $\mathcal{G}$ is weakly filtered. In order to prove that $\mathcal{G}$ is strongly filtered, we argue by induction on the length of a $\Sigma$-filtration of $\mathcal{G}_{F_{0}}$, this length being well-defined by Lemma 4.2.1. The case of length $\leq 1$ is clear. Otherwise, by the definition of being weakly filtered, there is a short exact sequence of finite flat $R$-group schemes (with descent data relative to $F_{0}$ )

$$
(0) \longrightarrow \mathcal{G}^{\prime} \longrightarrow \mathcal{G} \longrightarrow \mathcal{G}^{\prime \prime} \longrightarrow(0)
$$

with $\mathcal{G}^{\prime} \in \Sigma$ and $\mathcal{G}^{\prime \prime}$ weakly filtered (and hence, by inductive hypothesis, strongly filtered). Let $\mathcal{H}$ be any closed subgroup scheme of $\mathcal{G}$ (with compatible descent data relative to $F_{0}$ ) such that $\mathcal{H}_{F_{0}} \simeq \mathcal{G}_{i_{0}, F_{0}}$ for some $\mathcal{G}_{i_{0}} \in \Sigma$ and such that $(\mathcal{G} / \mathcal{H})_{F_{0}}$ admits a $\Sigma$-filtration. We need to prove (in the category of finite flat group schemes with descent data relative to $\left.F^{\prime} / F_{0}\right)$ that

- $\mathcal{H} \simeq \mathcal{G}_{i_{0}}$,

- and $\mathcal{G} / \mathcal{H}$ is weakly filtered.

If the composite map

$$
\mathcal{H} \hookrightarrow \mathcal{G} \longrightarrow \mathcal{G}^{\prime \prime}
$$

is zero, then $\mathcal{H}=\mathcal{G}^{\prime}$ as closed subgroup schemes of $\mathcal{G}$ (with descent data) and likewise $\mathcal{G} / \mathcal{H}=\mathcal{G}^{\prime \prime}$, so we are done. The interesting case is when the composite map is non-zero. The map $\mathcal{G}_{i_{0}, F_{0}} \cong \mathcal{H}_{F_{0}} \rightarrow \mathcal{G}_{F_{0}}^{\prime \prime}$ is then non-zero and therefore must be a closed immersion by the assumption (4.2.1) on $\Sigma$ and a devissage with respect to a $\Sigma$-filtration of $\mathcal{G}_{F_{0}}^{\prime \prime}$. We conclude that the map of generic fibre étale group schemes $\mathcal{H} \times F^{\prime} \rightarrow \mathcal{G}^{\prime \prime} \times F^{\prime}$ is a closed immersion.

Taking scheme-theoretic closures, we obtain a closed subgroup scheme $\mathcal{H}^{\prime \prime} \hookrightarrow \mathcal{G}^{\prime \prime}$ (with unique compatible descent data over $F_{0}$ ) fitting into a commutative diagram 
of group schemes with descent data

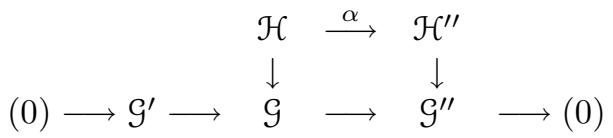

in which the lower row is short exact, the vertical maps are closed immersions and the top map $\mathcal{H} \rightarrow \mathcal{H}^{\prime \prime}$ induces an isomorphism on generic fibres. By Lemma 4.2.1 we may extend $\mathcal{H}_{F_{0}} \hookrightarrow \mathcal{G}_{F_{0}}^{\prime \prime}$ to a $\Sigma$-filtration on $\mathcal{G}_{F_{0}}^{\prime \prime}$ and so, because $\mathcal{G}^{\prime \prime}$ is strongly filtered by induction, we may extend $\mathcal{H}^{\prime \prime} \hookrightarrow \mathcal{G}^{\prime \prime}$ to a $\Sigma$-filtration. In particular $\mathcal{H}^{\prime \prime}$ is isomorphic to an object in $\Sigma$ and $\mathcal{G}^{\prime \prime} / \mathcal{H}^{\prime \prime}$ is strongly filtered.

Pulling back the short exact sequence

$$
0 \longrightarrow \mathcal{G}^{\prime} \longrightarrow \mathcal{G} \longrightarrow \mathcal{G}^{\prime \prime} \longrightarrow 0
$$

by $\mathcal{H}^{\prime \prime} \rightarrow \mathcal{G}^{\prime \prime}$, we get a diagram

$$
\begin{aligned}
& \mathcal{H} \\
& \downarrow \\
& 0 \longrightarrow \mathcal{G}^{\prime} \longrightarrow \mathcal{G} \times \mathcal{G}^{\prime \prime} \mathcal{H}^{\prime \prime} \longrightarrow \mathcal{H}^{\prime \prime} \longrightarrow 0
\end{aligned}
$$

in which the row is a short exact sequence of fppf abelian sheaves and all of the terms are finite flat group schemes (for the middle, this follows from the flatness of $\left.\mathcal{G} \rightarrow \mathcal{G}^{\prime \prime}\right)$. Thus, this bottom row is a short exact sequence of finite flat group schemes (with descent data). As $\mathcal{H}_{F_{0}} \stackrel{\sim}{\rightarrow} \mathcal{H}_{F_{0}}^{\prime \prime}$, the sequence

$$
0 \longrightarrow \mathcal{G}_{F_{0}}^{\prime} \longrightarrow\left(\mathcal{G} \times \mathcal{G}^{\prime \prime} \mathcal{H}^{\prime \prime}\right)_{F_{0}} \longrightarrow \mathcal{H}_{F_{0}}^{\prime \prime} \longrightarrow 0
$$

is split. In particular $\left(\mathcal{G} \times \mathcal{G}^{\prime \prime} \mathcal{H}^{\prime \prime}\right)_{F_{0}}$ and hence $\mathcal{G} \times \mathcal{G}^{\prime \prime} \mathcal{H}^{\prime \prime}$ are killed by $\ell$. By the hypothesis of the lemma

$$
0 \longrightarrow \mathcal{G}^{\prime} \longrightarrow \mathcal{G} \times \mathcal{G}^{\prime \prime} \mathcal{H}^{\prime \prime} \longrightarrow \mathcal{H}^{\prime \prime} \longrightarrow 0
$$

is also split, i.e. we have an isomorphism

$$
\mathcal{G} \times \mathcal{G}^{\prime \prime} \mathcal{H}^{\prime \prime} \cong \mathcal{G}^{\prime} \times_{R} \mathcal{H}^{\prime \prime}
$$

such that $\mathcal{G}^{\prime} \hookrightarrow \mathcal{G} \times \mathcal{G}^{\prime \prime} \mathcal{H}^{\prime \prime}$ corresponds to injection to the first factor of $\mathcal{G}^{\prime} \times_{R} \mathcal{H}^{\prime \prime}$ and $\mathcal{G} \times \mathcal{G}^{\prime \prime} \mathcal{H}^{\prime \prime} \rightarrow \mathcal{H}^{\prime \prime}$ corresponds to projection onto the second factor. By our hypotheses on $\Sigma$ we can find a morphism $\phi: \mathcal{H}^{\prime \prime} \rightarrow \mathcal{G}^{\prime}$ extending

$$
\mathcal{H}_{F_{0}}^{\prime \prime} \stackrel{\sim}{\longleftarrow} \mathcal{H}_{F_{0}} \hookrightarrow \mathcal{G}_{F_{0}}^{\prime} \times \mathcal{H}_{F_{0}}^{\prime \prime} \stackrel{\text { pr }}{\rightarrow} \mathcal{G}_{F_{0}}^{\prime}
$$

Then our closed immersion $\mathcal{H} \hookrightarrow \mathcal{G}^{\prime} \times_{R} \mathcal{H}^{\prime \prime}$ factors as

$$
\mathcal{H} \longrightarrow \mathcal{H}^{\prime \prime} \stackrel{\phi \times 1}{\longrightarrow} \mathcal{G}^{\prime} \times_{R} \mathcal{H}^{\prime \prime} .
$$

As $\mathcal{H} \rightarrow \mathcal{G}^{\prime} \times{ }_{R} \mathcal{H}^{\prime \prime}$ is a closed immersion, $\alpha: \mathcal{H} \rightarrow \mathcal{H}^{\prime \prime}$ must be a closed immersion and hence an isomorphism. Thus $\mathcal{H}$ is isomorphic to an object in $\Sigma$.

Now we turn to the proof that $\mathcal{G} / \mathcal{H}$ is weakly filtered. Since $\alpha: \mathcal{H} \rightarrow \mathcal{H}^{\prime \prime}$ is an isomorphism, it is clear that the natural map

$$
\mathcal{H} \times_{R} \mathcal{G}^{\prime} \longrightarrow \mathcal{G} \times \mathcal{G}^{\prime \prime} \mathcal{H}^{\prime \prime}
$$

is an isomorphism, and hence that

$$
\mathcal{H} \times_{R} \mathcal{G}^{\prime} \longrightarrow \mathcal{G}
$$

is a closed immersion. Thus, the finite flat group scheme $\mathcal{G} /\left(\mathcal{H} \times \mathcal{G}^{\prime}\right)$ makes sense and the natural map

$$
\mathcal{G} /\left(\mathcal{H} \times \mathcal{G}^{\prime}\right) \longrightarrow \mathcal{G}^{\prime \prime} / \mathcal{H}^{\prime \prime}
$$


is an isomorphism (as one sees by using the universal properties of quotients to construct an inverse map). We therefore arrive at a short exact sequence

$$
0 \longrightarrow \mathcal{G}^{\prime} \longrightarrow \mathcal{G} / \mathcal{H} \longrightarrow \mathcal{G}^{\prime \prime} / \mathcal{H}^{\prime \prime} \longrightarrow 0
$$

(compatible with descent data). Since $\mathcal{G}^{\prime \prime} / \mathcal{H}^{\prime \prime}$ is strongly filtered, as we noted above, and $\mathcal{G}^{\prime} \in \Sigma$, it follows that $\mathcal{G} / \mathcal{H}$ is weakly filtered.

Corollary 4.2.5. Fix $\mathcal{J}$ and $\Sigma$ as above. Suppose that $\Sigma=\{(\mathcal{G},\{[g]\})\}$ is a singleton. Suppose also that we have a short exact sequence

$$
(0) \longrightarrow\left(\mathcal{G}_{1},\{[g]\}\right) \longrightarrow(\mathcal{G},\{[g]\}) \longrightarrow\left(\mathcal{G}_{2},\{[g]\}\right) \longrightarrow(0)
$$

in $\mathcal{F} \mathcal{D}_{F^{\prime} / F_{0}, \mathcal{J}}$, where for any $i, j$ (possibly equal)

$$
\begin{aligned}
\operatorname{Hom}\left(\left(\mathcal{G}_{i},\{[g]\}\right),\left(\mathcal{G}_{j},\{[g]\}\right)\right) & =\operatorname{Hom}\left(\left(\mathcal{G}_{i},\{[g]\}\right)_{F_{0}},\left(\mathcal{G}_{j},\{[g]\}\right)_{F_{0}}\right) \\
& = \begin{cases}0 & \text { if } i \neq j, \\
\text { finite field } & \text { if } i=j,\end{cases}
\end{aligned}
$$

and the natural map

$$
\operatorname{Ext}_{\mathcal{F D}_{F^{\prime} / F_{0},(\mathcal{J}, \ell)}}^{1}\left(\left(\mathcal{G}_{i},\{[g]\}\right),\left(\mathcal{G}_{j},\{[g]\}\right)\right) \longrightarrow \operatorname{Ext}_{\mathbf{F}_{\ell}\left[G_{F_{0}}\right]}^{1}\left(\left(\mathcal{G}_{i},\{[g]\}\right)_{F_{0}},\left(\mathcal{G}_{j},\{[g]\}\right)_{F_{0}}\right)
$$

is injective. Then any object $(\mathcal{H},\{[g]\})$ of $\mathcal{F D}_{F^{\prime} / F_{0}, \mathcal{J}}$ which is weakly filtered by $\Sigma$ is also strongly filtered by $\Sigma$.

Proof. As $(\mathcal{H},\{[g]\})$ is weakly filtered by $\Sigma$, it is weakly filtered by $\left\{\left(\mathcal{G}_{1},\{[g]\}\right)\right.$, $\left.\left(\mathcal{G}_{2},\{[g]\}\right)\right\}$, and so by Lemma 4.2.4 is strongly filtered by $\left\{\left(\mathcal{G}_{1},\{[g]\}\right),\left(\mathcal{G}_{2},\{[g]\}\right)\right\}$. Any $\Sigma$-filtration of $(\mathcal{H},\{[g]\})_{F_{0}}$ extends to a $\left\{\left(\mathcal{G}_{1},\{[g]\}\right),\left(\mathcal{G}_{2},\{[g]\}\right)\right\}$-filtration of $(\mathcal{H},\{[g]\})_{F_{0}}$, which in turn gives rise to a $\left\{\left(\mathcal{G}_{1},\{[g]\}\right),\left(\mathcal{G}_{2},\{[g]\}\right)\right\}$-filtration of $(\mathcal{H},\{[g]\})$. By the injectivity of (4.2.2) we see that this yields a $\Sigma$-filtration of $(\mathcal{H},\{[g]\})$ that induces to our chosen $\Sigma$-filtration of $(\mathcal{H},\{[g]\})_{F_{0}}$.

4.3. Generalities on deformation theory. Again in this section $\ell$ denotes an arbitrary rational prime. We let $K$ denote a finite extension of $\mathbf{Q}_{\ell}, \mathcal{O}$ the ring of integers $K, \wp_{K}$ the maximal ideal of $\mathcal{O}$ and $k$ its residue field. Note that $k$ has a different meaning from the previous two sections. Let $V$ be a two-dimensional $k$-vector space and $\bar{\rho}: G_{\ell} \rightarrow \operatorname{Aut}_{k}(V)$ a continuous representation. Suppose that the centraliser of $G_{\ell}$ in $\operatorname{End}_{k}(V)$ is $k$. Let $\psi: G_{\ell} \rightarrow \mathcal{O}^{\times}$denote a continuous character such that $\left(\psi \bmod \wp_{K}\right) \cong \operatorname{det} \bar{\rho}$. Let $\mathcal{S}(\bar{\rho})$ denote the full subcategory of the category of finite length (discrete) $\mathcal{O}$-modules with a continuous $\mathcal{O}$-linear action of $G_{\ell}$ consisting of objects which admit a finite filtration so that each successive quotient is isomorphic to $V$. Because $\operatorname{End}_{k\left[G_{\ell}\right]}(V)=k$, it follows from the usual Jordan-Hölder argument that $\mathcal{S}(\bar{\rho})$ is an abelian category.

Let $\mathcal{S}$ be a full subcategory of $\mathcal{S}(\bar{\rho})$ stable under isomorphisms and which is closed under finite products, $\mathcal{S}(\bar{\rho})$-subobjects and $\mathcal{S}(\bar{\rho})$-quotients, and which contains $V$. We will consider the following set-valued functors on the category of complete noetherian local $\mathcal{O}$-algebras $R$ with finite residue field $k$.

- $\mathcal{D}_{V, \mathcal{O}}(R)$ is the set of conjugacy classes of continuous representations $\rho$ : $G_{\ell} \rightarrow \mathrm{GL}_{2}(R)$ such that $\rho \bmod \mathfrak{m}_{R}$ is conjugate to $\bar{\rho}$.

- $\mathcal{D}_{V, \mathcal{O}}^{\psi}(R)$ is the set of conjugacy classes of continuous representations $\rho$ : $G_{\ell} \rightarrow \mathrm{GL}_{2}(R)$ such that $\rho \bmod \mathfrak{m}_{R}$ is conjugate to $\bar{\rho}$ and $\operatorname{det} \rho=\psi$. 
- $\mathcal{D}_{V, \mathcal{O}}^{\mathcal{S}}(R)$ is the set of conjugacy classes of continuous representations $\rho$ : $G_{\ell} \rightarrow \mathrm{GL}_{2}(R)$ such that $\rho \bmod \mathfrak{m}_{R}$ is conjugate to $\bar{\rho}$ and such that for each open ideal $\mathfrak{a} \subset R$ the action $\rho$ makes $(R / \mathfrak{a})^{2}$ into an object of $\mathcal{S}$.

- $\mathcal{D}_{V, \mathcal{O}}^{\psi, \mathcal{S}}(R)$ is the set of conjugacy classes of continuous representations $\rho$ : $G_{\ell} \rightarrow \mathrm{GL}_{2}(R)$ such that $\rho \bmod \mathfrak{m}_{R}$ is conjugate to $\bar{\rho}$, such that $\operatorname{det} \rho=\psi$, and such that for each open ideal $\mathfrak{a} \subset R$ the action $\rho$ makes $(R / \mathfrak{a})^{2}$ into an object of $\mathcal{S}$.

Each of these deformation problems is representable by objects which we will denote $R_{V, \mathcal{O}}, R_{V, \mathcal{O}}^{\psi}, R_{V, \mathcal{O}}^{\mathcal{S}}$ and $R_{V, \mathcal{O}}^{\psi, \mathcal{S}}$, respectively.

Recall that the following sets are in natural ( $k$-linear) bijection with each other.

- $\left(\mathfrak{m}_{R_{V, \mathcal{O}}} /\left(\wp_{K}, \mathfrak{m}_{R_{V, \mathcal{O}}}^{2}\right)\right)^{\vee}$.

- The set of deformations of $\bar{\rho}$ to $k[\varepsilon] /\left(\varepsilon^{2}\right)$.

- $\operatorname{Ext}_{k\left[G_{\ell}\right]}^{1}(V, V)$.

- $H^{1}\left(G_{\ell}, \operatorname{ad} \bar{\rho}\right)$.

These bijections give rise to an isomorphism

$$
\left(\mathfrak{m}_{R_{V, \mathcal{O}}^{\psi}} /\left(\wp_{K}, \mathfrak{m}_{R_{V, \mathcal{O}}^{\psi}}^{2}\right)\right)^{\vee} \cong H^{1}\left(G_{\ell}, \operatorname{ad}^{0} \bar{\rho}\right),
$$

as well as bijections between

- $\left(\mathfrak{m}_{R_{V, \mathcal{O}}^{s}} /\left(\wp_{K}, \mathfrak{m}_{R_{V, \mathcal{O}}^{s}}^{2}\right)\right)^{\vee}$,

- the set of deformations of $\bar{\rho}$ to $k[\varepsilon] /\left(\varepsilon^{2}\right)$ which make $\left(k[\varepsilon] /\left(\varepsilon^{2}\right)\right)^{2}$ into an object of $\mathcal{S}$,

- $\operatorname{Ext}_{k\left[G_{\ell}\right], s}^{1}(V, V)$, i.e. $\operatorname{Ext}^{1}$ in the category of discrete $k\left[G_{\ell}\right]$-modules which are also objects of $\mathcal{S}$,

- the subgroup $H_{\mathcal{S}}^{1}\left(G_{\ell}, \operatorname{ad} \bar{\rho}\right) \subset H^{1}\left(G_{\ell}, \operatorname{ad} \bar{\rho}\right)$ corresponding to $\operatorname{Ext}_{k\left[G_{\ell}\right], \mathcal{S}}^{1}(V, V)$.

We will set $H_{\mathcal{S}}^{1}\left(G_{\ell}, \operatorname{ad}^{0} \bar{\rho}\right)=H_{\mathcal{S}}^{1}\left(G_{\ell}, \operatorname{ad} \bar{\rho}\right) \cap H^{1}\left(G_{\ell}, \operatorname{ad}^{0} \bar{\rho}\right)$, so that we get an isomorphism

$$
\left(\mathfrak{m}_{R_{V, \mathcal{O}}^{\psi, \mathcal{S}}} /\left(\wp_{K}, \mathfrak{m}_{R_{V, \mathcal{O}}^{\psi, S}}^{2}\right)\right)^{\vee} \cong H_{\mathcal{S}}^{1}\left(G_{\ell}, \operatorname{ad}^{0} \bar{\rho}\right) .
$$

4.4. Reduction steps for Theorem 2.1.2, We now begin the proof of Theorem 2.1.2 Making an unramified twist we may suppose that $\bar{\rho}$ has the form

$$
\left(\begin{array}{ll}
1 & * \\
0 & \omega
\end{array}\right)
$$

We may also suppose that $\mathcal{O}=\mathbf{Z}_{3}$.

Let $F_{1}=F_{1}^{\prime}$ denote a totally ramified cubic Galois extension of $\mathbf{Q}_{3}$. Let $F_{-1}^{\prime}$ denote the unique cubic extension of $\mathbf{Q}_{3}(\sqrt{-1})$ such that $F_{-1}^{\prime} / \mathbf{Q}_{3}$ is Galois but not abelian, and let $F_{-1}$ denote a cubic subfield of $F_{-1}^{\prime}$, so $F_{-1}^{\prime} / F_{-1}$ is unramified.

Let $\mathcal{S}_{ \pm 1}$ denote the full subcategory $\mathcal{S}(\bar{\rho})$ consisting of $\mathbf{Z}_{3}\left[G_{3}\right]$-modules $X$ for which there exists a finite flat $\mathcal{O}_{F_{ \pm 1}^{\prime}}$-group scheme $(\mathcal{G},\{[g]\})$ with descent data for $F_{ \pm 1}^{\prime} / \mathbf{Q}_{3}$ such that $X \cong(\mathcal{G},\{[g]\})_{\mathbf{Q}_{3}}\left(\overline{\mathbf{Q}}_{3}\right)$ as a $\mathbf{Z}_{3}\left[G_{3}\right]$-module. By Lemma 4.1.3 we see that $S_{ \pm 1}$ is closed under finite products, subobjects and quotients. Using Tate's theorem on the uniqueness of extensions of 3-divisible groups from $F_{ \pm 1}^{\prime}$ to $\mathcal{O}_{F_{ \pm 1}^{\prime}}$ (Theorem 4 of [T]), we see that the map $R_{V, \mathbf{Z}_{3}} \rightarrow R_{V, \mathbf{Z}_{3}}^{\tau_{ \pm 1}}$ factors through $R_{V, \mathbf{Z}_{3}}^{\epsilon, \mathcal{S}_{ \pm 1}}$. Thus, Theorem 2.1.2 follows from the following result which we will prove in $₫ 7$

Theorem 4.4.1. $\operatorname{dim} H_{\mathcal{S}_{ \pm 1}}^{1}\left(G_{3}, \operatorname{ad}^{0} \bar{\rho}\right) \leq 1$. 
4.5. Reduction steps for Theorem 2.1.4, We now begin the proof of Theorem 2.1.4. Making an unramified twist, we may suppose that $\bar{\rho}$ has the form

$$
\left(\begin{array}{ll}
\omega & * \\
0 & 1
\end{array}\right) \text {. }
$$

We may also suppose that $\mathcal{O}=\mathbf{Z}_{3}$.

Let $F_{ \pm 3}^{\prime}$ denote the degree 12 abelian extension of $\mathbf{Q}_{3}(\sqrt{ \pm 3})$ with norm subgroup in $\mathbf{Q}_{3}(\sqrt{ \pm 3}) \times$ topologically generated by $\pm 3,4$ and $1+3 \sqrt{ \pm 3}$. Note that $F_{ \pm 3}^{\prime} / \mathbf{Q}_{3}$ is Galois. We have an isomorphism

$$
\operatorname{Gal}\left(F_{ \pm 3}^{\prime} / \mathbf{Q}_{3}(\sqrt{ \pm 3})\right) \cong C_{2} \times C_{2} \times C_{3} .
$$

Let $\gamma_{4}^{2} \in I_{F_{ \pm 3}^{\prime} / \mathbf{Q}_{3}(\sqrt{ \pm 3})}$ be the unique element of order 2. (In later applications this will be the square of an element of order 4 in $\operatorname{Gal}\left(F_{ \pm 3}^{\prime} / \mathbf{Q}_{3}\right)$.) We also let $F_{ \pm 3}$ denote the fixed field of a Frobenius lift of order 2 , so $F_{ \pm 3} / \mathbf{Q}_{3}$ is totally ramified.

We will let $\mathcal{J}_{ \pm 3}$ denote the two-sided ideal of $W\left(\mathbf{F}_{9}\right)[\mathbf{F}, \mathbf{V}]\left[\mathrm{Gal}\left(F_{ \pm 3}^{\prime} / \mathbf{Q}_{3}\right)\right]$ generated by

- $\mathbf{F}+\mathbf{V}$

- and $\left[\gamma_{4}^{2}\right]+1$.

Let $\mathcal{S}_{ \pm 3}$ denote the full subcategory of $\mathcal{S}(\bar{\rho})$ consisting of objects $X$ for which we can find an object $(\mathcal{G},\{[g]\})$ of $\mathcal{F D}_{F_{ \pm 3}^{\prime} / \mathbf{Q}_{3}, \mathcal{J}_{ \pm 3}}$ such that $X \cong(\mathcal{G},\{[g]\})_{\mathbf{Q}_{3}}\left(\overline{\mathbf{Q}}_{3}\right)$ as a $\mathbf{Z}_{3}\left[G_{3}\right]$-module. By Lemma 4.1.3, we see that $\mathcal{S}_{ \pm 3}$ is closed under finite products, subobjects and quotients.

Now choose a finite extension $K / \mathbf{Q}_{3}$ and continuous map of rings $f: R_{V, \mathbf{Z}_{3}} \rightarrow \overline{\mathbf{Q}}_{3}$ such that the corresponding representation $\rho: G_{3} \rightarrow \mathrm{GL}_{2}\left(\mathcal{O}_{K}\right)$ is of type $\tau_{ \pm 3}$. Let $G$ be the corresponding 3-divisible group over $\mathbf{Q}_{3}$. By Tate's theorem (Theorem 4 of [T] ), the base change of $G$ to $F_{ \pm 3}^{\prime}$ has a unique extension to a 3-divisible group $\mathcal{G}$ over $\mathcal{O}_{F_{ \pm 3}^{\prime}}$. By the uniqueness of this extension, it is also equipped with descent data $\{[g]\}$ relative to $F_{ \pm 3}^{\prime} / \mathbf{Q}_{3}$ and with an action of $\mathcal{O}_{K}$, compatible with the canonical structure on the generic fibre.

Let $\widetilde{\gamma}_{2} \in \operatorname{Gal}\left(\mathbf{Q}_{3}(\sqrt{ \pm 3})^{\text {ab }} / \mathbf{Q}_{3}(\sqrt{ \pm 3})\right)$ correspond to $\sqrt{ \pm 3}$. We will use the notation of Appendix B of [CDT] (in particular WD and $D^{\prime}(\mathcal{G})$ ), except that we will write $\mathbf{F}$ and $\mathbf{F}^{\prime}$ in place of $\phi$ and $\phi^{\prime}$. Then

- $\mathrm{WD}(\rho)\left(\gamma_{4}^{2}\right)=-1$,

- $\mathrm{WD}(\rho)\left(\widetilde{\gamma}_{2}^{2}\right)$, but not $\mathrm{WD}(\rho)\left(\widetilde{\gamma}_{2}\right)$, is a scalar,

- and $\operatorname{det} \operatorname{WD}(\rho)\left(\widetilde{\gamma}_{2}\right)=3$.

Thus $\mathrm{WD}(\rho)\left(\widetilde{\gamma}_{2}^{2}\right)=-3$. Hence on $D^{\prime}(\mathcal{G}) \otimes \mathbf{Q}_{3}$ we have

- $\left[\gamma_{4}^{2}\right]=\mathrm{WD}(\rho)\left(\gamma_{4}^{2}\right)=-1$,

- and $\left(\mathbf{F}^{\prime}\right)^{2}=\left[\widetilde{\gamma}_{2}^{2}\right] \mathrm{WD}(\rho)\left(\widetilde{\gamma}_{2}^{-2}\right)=-1 / 3$.

We conclude that on $D(\mathcal{G})$ we have

- $\left[\gamma_{4}^{2}\right]=-1$,

- $\mathbf{F}^{2}=-3$,

- and so $\mathbf{F}=-\mathbf{V}$.

In particular $\mathcal{J}_{ \pm 3}$ annihilates $D(\mathcal{G})$ and for all $m \geq 1$ the $\operatorname{map}\left(f \bmod 3^{m}\right): R_{V, \mathbf{Z}_{3}} \rightarrow$ $\mathcal{O}_{K} /\left(3^{m}\right)$ factors through $R_{V, \mathbf{Z}_{3}}^{\epsilon, \mathcal{S}_{ \pm 3}}$. Hence, the map $R_{V, \mathbf{Z}_{3}} \rightarrow R_{V, \mathbf{Z}_{3}}^{\tau_{ \pm 3}}$ factors through $R_{V, \mathbf{Z}_{3}}^{\epsilon, \mathcal{S}_{ \pm 3}}$ and Theorem 2.1 .4 follows from the following result which we will prove in §. 
Theorem 4.5.1. $\operatorname{dim} H_{\mathcal{S}_{ \pm 3}}^{1}\left(G_{3}, \operatorname{ad}^{0} \bar{\rho}\right) \leq 1$.

4.6. Reduction steps for Theorem 2.1.6. We now begin the proof of Theorem 2.1.6. We may suppose that $\mathcal{O}=\mathbf{Z}_{3}$.

Let $F_{i}^{\prime}$ denote the degree 12 abelian extension of $\mathbf{Q}_{3}(\sqrt{-3})$ with norms the subgroup of $\mathbf{Q}_{3}(\sqrt{-3})^{\times}$topologically generated by $-3,4,1+9 \sqrt{-3}$ and $1+$ $(1-3 \tilde{\imath}) \sqrt{-3}$, where $\tilde{\imath}$ is the unique lift of $i$ to $\mathbf{Z}$ with $0 \leq \tilde{\imath}<3$. Note that $F_{i}^{\prime} / \mathbf{Q}_{3}$ is Galois. We identify

$$
\operatorname{Gal}\left(F_{i}^{\prime} / \mathbf{Q}_{3}(\sqrt{-3})\right) \cong\left\langle\gamma_{2}\right\rangle \times\left\langle\gamma_{3}\right\rangle \times\left\langle\gamma_{4}^{2}\right\rangle,
$$

where $\gamma_{2}$ corresponds to $\sqrt{-3}$ and has order $2, \gamma_{3}$ corresponds to $1-3 \sqrt{-3}$ and has order 3, and $\gamma_{4}^{2}$ corresponds to -1 and has order 2 . We also let $F_{i}$ denote the fixed field of $\left\{1, \gamma_{2}\right\}$, so $F_{i} / \mathbf{Q}_{3}$ is totally ramified.

We will let $\mathcal{J}_{i}$ denote the two-sided ideal of $W\left(\mathbf{F}_{9}\right)[\mathbf{F}, \mathbf{V}]\left[\operatorname{Gal}\left(F_{i}^{\prime} / \mathbf{Q}_{3}\right)\right]$ generated by

$$
\begin{aligned}
& -\mathbf{F}+\mathbf{V} \\
& \text { - }\left[\gamma_{4}^{2}\right]+1, \\
& \text { - and }\left(\left[\gamma_{3}\right]-\left[\gamma_{3}^{-1}\right]\right)\left[\gamma_{2}\right]-\mathbf{F} \text {. }
\end{aligned}
$$

We remark that the ideal $\mathcal{J}_{i}$ is unchanged if we change our choice of $\sqrt{-3}$.

In $₫ 9$ we will prove the following result (and explain the unusual looking notation).

Theorem 4.6.1. There are objects $(\mathcal{G},\{[g]\})_{(2,6)},(\mathcal{G},\{[g]\})_{(6,10)},(\mathcal{G},\{[g]\})_{(2,10)}$ and $(\mathcal{G},\{[g]\})_{(6,6)}$ in the category $\mathcal{F D}_{F_{i}^{\prime} / \mathbf{Q}_{3}, \mathcal{J}_{i}}$ with the following properties.

(1) For $(r, s)=(2,6),(6,10),(2,10)$ and $(6,6)$ we have $\bar{\rho} \cong\left((\mathcal{G},\{[g]\})_{(r, s)}\right)_{\mathbf{Q}_{3}}\left(\overline{\mathbf{Q}}_{3}\right)$ as $G_{3}$-modules.

(2) For $(r, s)=(2,6),(6,10),(2,10)$ and $(6,6)$ there is a short exact sequence in $\mathcal{F D}_{F_{i}^{\prime} / \mathbf{Q}_{3}, \mathfrak{J}_{i}}$,

$$
(0) \longrightarrow\left(\mathcal{G}_{1},\{[g]\}\right)_{(r, s)} \longrightarrow(\mathcal{G},\{[g]\})_{(r, s)} \longrightarrow\left(\mathcal{G}_{2},\{[g]\}\right)_{(r, s)} \longrightarrow(0),
$$

such that $\left(\mathcal{G}_{1},\{[g]\}\right)_{(r, s)}$ and $\left(\mathcal{G}_{2},\{[g]\}\right)_{(r, s)}$ have order 3 and for all $a, b \in$ $\{1,2\}$ (possibly equal) the natural map

$$
\begin{aligned}
\operatorname{Ext}_{\mathcal{F}_{\mathcal{D}^{\prime} / \mathbf{Q}_{3},\left(\mathcal{J}_{i}, 3\right)}}^{1}\left(\left(\mathcal{G}_{a},\{[g]\}\right)_{(r, s)},\left(\mathcal{G}_{b},\{[g]\}\right)\right)_{(r, s)} & \\
& \longrightarrow \operatorname{Ext}_{\mathbf{F}_{3}\left[G_{\mathbf{Q}_{3}}\right]}^{1}\left(\left(\mathcal{G}_{a},\{[g]\}\right)_{(r, s), \mathbf{Q}_{3}},\left(\mathcal{G}_{b},\{[g]\}\right)_{(r, s), \mathbf{Q}_{3}}\right)
\end{aligned}
$$

is injective.

(3) If $k / \mathbf{F}_{3}$ is a finite field extension and if $(\mathcal{G},\{[g]\})$ is an object of $\mathcal{F} \mathcal{D}_{F_{i}^{\prime} / \mathbf{Q}_{3}, \mathcal{J}_{i}}$ with an action of $k$ such that $(\mathcal{G},\{[g]\})_{\mathbf{Q}_{3}}\left(\overline{\mathbf{Q}}_{3}\right)$ is isomorphic to $\bar{\rho} \otimes k$, then for some $(r, s)=(2,6),(6,10),(2,10)$ or $(6,6)$ the object $(\mathcal{G},\{[g]\})$ of $\mathcal{F D}_{F_{i}^{\prime} / \mathbf{Q}_{3}, \mathcal{J}_{i}}$ is weakly filtered by $\left\{\left(\mathcal{G}_{1},\{[g]\}\right)_{(r, s)},\left(\mathcal{G}_{2},\{[g]\}\right)_{(r, s)}\right\}$.

(4) For $(r, s)=(2,6),(6,10)$ and $(2,10)$ we have $\mathbf{F}=0$ on $D\left(\mathcal{G}_{(r, s)}\right)$, while $\mathbf{F} \neq 0$ on $D\left(\mathcal{G}_{(6,6)}\right)$.

Note that for all $a, b$ (possibly equal), we must have

$$
\begin{aligned}
\operatorname{Hom}\left(\left(\mathcal{G}_{a},\{[g]\}\right)_{(r, s)},\left(\mathcal{G}_{b},\{[g]\}\right)_{(r, s)}\right) & =\operatorname{Hom}\left(\left(\mathcal{G}_{a},\{[g]\}\right)_{(r, s), \mathbf{Q}_{3}},\left(\mathcal{G}_{b},\{[g]\}\right)_{(r, s), \mathbf{Q}_{3}}\right) \\
& = \begin{cases}0 & \text { if } a \neq b, \\
\mathbf{F}_{3} & \text { if } a=b .\end{cases}
\end{aligned}
$$


For $(r, s)=(2,6),(6,10),(2,10)$ and $(6,6)$, we let $\mathcal{S}_{i,(r, s)}$ denote the full subcategory of $\mathcal{S}(\bar{\rho})$ consisting of objects $X$ which are isomorphic to $(\mathcal{H},\{[g]\})_{\mathbf{Q}_{3}}$ for some object $(\mathcal{H},\{[g]\})$ of $\mathcal{F D}_{F_{i}^{\prime} / \mathbf{Q}_{3}, \mathcal{J}_{i},\left\{(\mathcal{G},\{[g]\})_{(r, s)}\right\}}$. By Lemma 4.2.2 Corollary 4.2 .5 and Theorem 4.6.1 we see that $\mathcal{S}_{i,(r, s)}$ is closed under finite products, $\mathcal{S}(\bar{\rho})$-subobjects and $\mathcal{S}(\bar{\rho})$-quotients. In $₫ 9$ we will also prove the following two results.

Theorem 4.6.2. For $(r, s)=(2,6),(6,10),(2,10)$ and $(6,6)$ we have

$$
\operatorname{dim} H_{\mathcal{S}_{i,(r, s)}}^{1}\left(G_{3}, \operatorname{ad}^{0} \bar{\rho}\right) \leq 1 .
$$

Theorem 4.6.3. For $(r, s)=(2,6),(6,10)$ and $(2,10)$ and for any $N \geq 1$ there exists a continuous representation

$$
\rho_{N}: G_{\mathbf{Q}_{3}} \longrightarrow \mathrm{GL}_{2}\left(\mathbf{F}_{3}[[T]] /\left(T^{N}\right)\right)
$$

such that

- $\operatorname{det} \rho_{N}=\epsilon$,

- for some object $\left(\mathcal{G}_{N},\{[g]\}\right)$ of $\mathcal{F D}_{F_{i}^{\prime} / \mathbf{Q}_{3},\left(\mathcal{J}_{i}, \mathbf{F}\right),\left\{(\mathcal{G},\{[g]\})_{(r, s)}\right\}}$ we have

$$
\rho_{N} \cong\left(\mathcal{G}_{N},\{[g]\}\right)_{\mathbf{Q}_{3}}\left(\overline{\mathbf{Q}}_{3}\right)
$$

(where $\left(\mathcal{J}_{i}, \mathbf{F}\right)$ denotes the two-sided ideal of $W\left(\mathbf{F}_{9}\right)[\mathbf{F}, \mathbf{V}]\left[\operatorname{Gal}\left(F_{i}^{\prime} / \mathbf{Q}_{3}\right)\right]$ generated by $\mathcal{J}_{i}$ and $\left.\mathbf{F}\right)$,

- and $\rho \bmod \left(T^{2}\right) \neq \bar{\rho} \otimes k[[T]] /\left(T^{2}\right)$.

(We are not asserting that $\rho_{N}$ and $\mathcal{G}_{N}$ are independent of the choice of $(r, s)$, though in fact we believe that $\rho_{N}$ is independent of this choice.)

From these results we can easily draw the following consequence.

Corollary 4.6.4. For $(r, s)=(2,6),(6,10)$ and $(2,10)$ we have

$$
R_{V, \mathbf{Z}_{3}}^{\epsilon, \mathcal{S}_{i,(r, s)}} \cong \mathbf{F}_{3}[[T]] \text {. }
$$

Proof. By Theorems 4.6.2 and 4.6.3 we see that $R_{V, \mathbf{Z}_{3}}^{\epsilon, \mathcal{S}_{i,(r, s)}} /(3) \cong \mathbf{F}_{3}[[T]]$ and that if $R$ is an Artinian quotient of $R_{V, \mathbf{Z}_{3}}^{\epsilon,(r, s)} /(3)$ corresponding to a (necessarily unique; see Lemma 4.2.3) object $(\mathcal{G},\{[g]\})$ of $\mathcal{F D}_{F_{i}^{\prime} / \mathbf{Q}_{3},\left(\mathcal{I}_{i}, 3\right),\left\{(\mathcal{G},\{[g]\})_{(r, s)}\right\}}$, then $\mathbf{F}=0$ on $D(\mathcal{G})$.

Now suppose $R$ is any Artinian quotient of $R_{V, \mathbf{Z}_{3}}^{\epsilon, \mathcal{S}_{i,(r, s)}}$ which corresponds to an $\operatorname{object}(\mathcal{G},\{[g]\})$ of the category $\mathcal{F D}_{F_{i}^{\prime} / \mathbf{Q}_{3}, \mathcal{J}_{i},\left\{(\mathcal{G},\{[g]\})_{(r, s)}\right\}}$. Let $G=(\mathcal{G},\{[g]\})_{\mathbf{Q}_{3}}$ and consider the exact sequences

$$
(0) \longrightarrow G[3] \longrightarrow G \longrightarrow 3 G \longrightarrow(0)
$$

and

$$
(0) \longrightarrow 3 G \longrightarrow G \longrightarrow G / 3 G \longrightarrow(0) .
$$

By Lemma 4.2.3, we have exact sequences

$$
(\mathcal{G},\{[g]\}) \longrightarrow(\mathcal{K},\{[g]\}) \longrightarrow(0)
$$

and

$$
(0) \longrightarrow(\mathcal{K},\{[g]\}) \longrightarrow(\mathcal{G},\{[g]\}) \longrightarrow(\mathcal{H},\{[g]\}) \longrightarrow(0)
$$

in $\mathcal{F D}_{F_{i}^{\prime} / \mathbf{Q}_{3}, \mathcal{J}_{i},\left\{(\mathcal{G},\{[g]\})_{(r, s)}\right\}}$ such that the composite

$$
(\mathcal{G},\{[g]\}) \rightarrow(\mathcal{K},\{[g]\}) \hookrightarrow(\mathcal{G},\{[g]\})
$$


is multiplication by 3 . In particular we have exact sequences

$$
(0) \longrightarrow D(\mathcal{K}) \longrightarrow D(\mathcal{G})
$$

and

$$
(0) \longrightarrow D(\mathcal{H}) \longrightarrow D(\mathcal{G}) \longrightarrow D(\mathcal{K}) \longrightarrow(0),
$$

such that the composite

$$
D(\mathcal{G}) \rightarrow D(\mathcal{K}) \hookrightarrow D(\mathcal{G})
$$

is multiplication by 3. As $\mathbf{F}=-\mathbf{V}=0$ on $D(\mathcal{H})$ we see that $\mathbf{F}$ and $\mathbf{V}$ factor through maps $D(\mathcal{K}) \rightarrow D(\mathcal{G})$, i.e. we can write $\mathbf{F}=3 \mathbf{F}^{\prime}$ and $\mathbf{V}=3 \mathbf{V}^{\prime}$ for some endomorphisms $\mathbf{F}^{\prime}$ and $\mathbf{V}^{\prime}$ of $D(\mathcal{G})$. Thus $3=9 \mathbf{F}^{\prime} \mathbf{V}^{\prime}$ equals zero on $D(\mathcal{G}) / 9 D(\mathcal{G})$ and so $D(\mathcal{K})=0$. We conclude that $\mathcal{K}=(0)$, so that $3 G=(0)$ and $3 R=(0)$.

Thus

$$
R_{V, \mathbf{Z}_{3}}^{\epsilon, \mathcal{S}_{i,(r, s)}}=R_{V, \mathbf{Z}_{3}}^{\epsilon, \mathcal{S}_{i,(r, s)}} /(3)=\mathbf{F}_{3}[[T]]
$$

We now modify the argument in $\$ 4.4$. Choose a finite extension $K / \mathbf{Q}_{3}$ and continuous map of rings $f: R_{V, \mathbf{Z}_{3}} \rightarrow \overline{\mathbf{Q}}_{3}$ such that the corresponding representation $\rho: G_{3} \rightarrow \mathrm{GL}_{2}\left(\mathcal{O}_{K}\right)$ is of extended type $\tau_{i}^{\prime}$. Let $G$ be the corresponding 3 -divisible group over $\mathbf{Q}_{3}$. By Tate's theorem (Theorem 4 of [T]) $G$ has a unique extension to a 3-divisible group $\mathcal{G}$ over $\mathcal{O}_{F_{i}^{\prime}}$. By the uniqueness of this extension, $\mathcal{G}$ comes equipped with descent data $\{[g]\}$ relative to $F_{i}^{\prime} / \mathbf{Q}_{3}$ and with an action of $\mathcal{O}_{K}$, compatible with the canonical structure on the generic fibre.

Let $\widetilde{\gamma}_{2} \in \operatorname{Gal}\left(\mathbf{Q}_{3}(\sqrt{-3})^{\mathrm{ab}} / \mathbf{Q}_{3}(\sqrt{-3})\right)$ correspond to $\sqrt{-3}$. We will use the notation of Appendix B of [CDT] (in particular WD and $D^{\prime}(\mathcal{G})$ ), except that we will write $\mathbf{F}$ and $\mathbf{F}^{\prime}$ in place of $\phi$ and $\phi^{\prime}$. Then

- $\mathrm{WD}(\rho)\left(\gamma_{4}^{2}\right)=-1$

- $\mathrm{WD}(\rho)\left(\widetilde{\gamma}_{2}^{2}\right)=-3$,

- and $\mathrm{WD}(\rho)\left(\widetilde{\gamma}_{2}\right)\left(\mathrm{WD}(\rho)\left(\gamma_{3}\right)-\mathrm{WD}(\rho)\left(\gamma_{3}\right)^{-1}\right)=3$.

Thus on $D^{\prime}(\mathcal{G}) \otimes \mathbf{Q}_{3}$ we have

- $\left[\gamma_{4}^{2}\right]=\mathrm{WD}(\rho)\left(\gamma_{4}^{2}\right)=-1$,

- $\left(\mathbf{F}^{\prime}\right)^{2}=\left[\widetilde{\gamma}_{2}^{2}\right] \mathrm{WD}(\rho)\left(\widetilde{\gamma}_{2}^{-2}\right)=-1 / 3$,

- and $\left[\gamma_{2}\right]\left(\left[\gamma_{3}\right]-\left[\gamma_{3}^{-1}\right]\right)=3 \mathbf{F}^{\prime}$.

We conclude that on $D(\mathcal{G})$ we have

- $\left[\gamma_{4}^{2}\right]=-1$,

- $\mathbf{F}^{2}=-3$,

- and $\left[\gamma_{2}\right]\left(\left[\gamma_{3}^{-1}\right]-\left[\gamma_{3}\right]\right)=3 \mathbf{F}^{-1}$.

Hence also

- $\mathbf{F}=-\mathbf{V}$,

- and $\left[\gamma_{2}\right]\left(\left[\gamma_{3}\right]-\left[\gamma_{3}^{-1}\right]\right)=\mathbf{F}$.

In particular $\mathcal{J}_{i}$ annihilates $D(\mathcal{G})$.

Thus $\left(\mathcal{G}\left[\wp_{K}\right],\{[g]\}\right)$ is an object of $\mathcal{F D}_{F_{i}^{\prime} / \mathbf{Q}_{3}, \mathcal{J}_{i}}$ such that $\left(\mathcal{G}\left[\wp_{K}\right],\{[g]\}\right)_{\mathbf{Q}_{3}}$ corresponds to $\bar{\rho} \otimes \mathcal{O}_{K} / \wp_{K}$. By Theorem 4.6.1 we see that $\left(\mathcal{G}\left[\wp_{K}\right],\{[g]\}\right)$ is weakly filtered by $\left\{\left(\mathcal{G}_{1},\{[g]\}\right)_{(r, s)},\left(\mathcal{G}_{2},\{[g]\}\right)_{(r, s)}\right\}$ for some $(r, s)=(2,6),(6,10),(2,10)$ or $(6,6)$. We will prove $(r, s)=(6,6)$. By Theorem 4.6.1 and Lemma 4.2.4 $\left(\mathcal{G}\left[\wp_{K}\right],\{[g]\}\right)$ 
is strongly filtered by $\left\{\left(\mathcal{G}_{1},\{[g]\}\right)_{(r, s)},\left(\mathcal{G}_{2},\{[g]\}\right)_{(r, s)}\right\}$. As $\left(\mathcal{G}\left[\wp_{K}\right],\{[g]\}\right)_{\mathbf{Q}_{3}}$ is filtered by $\bar{\rho}$, using Theorem 4.6 .1 we see that $\left(\mathcal{G}\left[\wp_{K}\right],\{[g]\}\right)$ is weakly filtered by $(\mathcal{G},\{[g]\})_{(r, s)}$. For all $m \geq 1$ we have

$$
\left(\mathcal{G}\left[\wp_{K}^{m}\right] / \mathcal{G}\left[\wp_{K}^{m-1}\right],\{[g]\}\right) \stackrel{\sim}{\longrightarrow}\left(\mathcal{G}\left[\wp_{K}\right],\{[g]\}\right),
$$

so for all $m \geq 1$ the object $\left(\mathcal{G}\left[\wp_{K}^{m}\right],\{[g]\}\right)$ is also weakly and hence strongly filtered by $(\mathcal{G},\{[g]\})_{(r, s)}$ for the same $(r, s)$. Thus, for all $m \geq 1$, the map $\left(f \bmod p^{m}\right)$ : $R_{V, \mathbf{Z}_{3}} \rightarrow \mathcal{O}_{K^{\prime}} /\left(3^{m}\right)$ factors through $R_{V, \mathbf{Z}_{3}}^{\epsilon, \mathcal{S}_{(r, s)}}$. By Corollary 4.6.4 we see that $(r, s)=(6,6)$, so the map $R_{V, \mathbf{Z}_{3}} \rightarrow R_{V, \mathbf{Z}_{3}}^{\tau_{i}^{\prime}}$ factors through $R_{V, \mathbf{Z}_{3}}^{\epsilon, \mathcal{S}_{i,(6,6)}}$ and Theorem 2.1.6 follows from Theorem 4.6.2,

4.7. Some Galois cohomology. In this section we will begin the proofs of Theorems 4.4.1, 4.5.1 and 4.6.2. We will let $\mathcal{S}$ denote one of the categories $\mathcal{S}_{ \pm 1}, \mathcal{S}_{ \pm 3}$ or $\mathcal{S}_{i,(r, s)}$. We will let $\chi=\omega$ in the cases $\mathcal{S}_{ \pm 1}$ and $\chi=1$ otherwise. In all cases

$$
\bar{\rho} \sim\left(\begin{array}{cc}
\chi \omega & * \\
0 & \chi
\end{array}\right)
$$

is très ramifié.

The maps $\omega \otimes \chi \hookrightarrow \bar{\rho}$ and $\bar{\rho} \rightarrow \chi$ induce a commutative diagram with exact rows and columns:

$$
\begin{aligned}
& \operatorname{Ext}_{\mathbf{F}_{3}\left[G_{3}\right]}^{1} \stackrel{\downarrow}{\downarrow}(\omega \otimes \chi, \omega \otimes \chi) \\
& (0) \longrightarrow \operatorname{Ext}_{\mathbf{F}_{3}\left[G_{3}\right]}^{1}(\chi, \chi) \longrightarrow \operatorname{Ext}_{\mathbf{F}_{3}\left[G_{3}\right]}^{1}(\bar{\rho}, \chi) \longrightarrow \operatorname{Ext}_{\mathbf{F}_{3}\left[G_{3}\right]}^{1}(\omega \otimes \chi, \chi)
\end{aligned}
$$

We will let $\theta_{0}$ denote the composite map

$$
\operatorname{Ext}_{\mathbf{F}_{3}\left[G_{3}\right]}^{1}(\bar{\rho}, \bar{\rho}) \longrightarrow \operatorname{Ext}_{\mathbf{F}_{3}\left[G_{3}\right]}^{1}(\omega \otimes \chi, \chi),
$$

and $\theta_{1}$ (resp. $\theta_{\omega}$ ) the induced mapping

$$
\operatorname{ker} \theta_{0} \longrightarrow \operatorname{Ext}_{\mathbf{F}_{3}\left[G_{3}\right]}^{1}(\chi, \chi)
$$

(resp.

$$
\left.\operatorname{ker} \theta_{0} \longrightarrow \operatorname{Ext}_{\mathbf{F}_{3}\left[G_{3}\right]}^{1}(\omega \otimes \chi, \omega \otimes \chi)\right) .
$$

We will also let $\bar{\theta}_{1}$ (resp. $\bar{\theta}_{\omega}$ ) denote the induced mapping

$$
\operatorname{ker} \theta_{0} \longrightarrow \operatorname{Ext}_{\mathbf{F}_{3}\left[G_{3}\right]}^{1}(\chi, \chi) \longrightarrow \operatorname{Ext}_{\mathbf{F}_{3}\left[I_{3}\right]}^{1}(\chi, \chi)
$$

(resp.

$$
\left.\operatorname{ker} \theta_{0} \longrightarrow \operatorname{Ext}_{\mathbf{F}_{3}\left[G_{3}\right]}^{1}(\omega \otimes \chi, \omega \otimes \chi) \longrightarrow \operatorname{Ext}_{\mathbf{F}_{3}\left[I_{3}\right]}^{1}(\omega \otimes \chi, \omega \otimes \chi)\right) .
$$


If we reinterpret our Ext-groups as cohomology groups and use the isomorphism $\bar{\rho}^{\vee} \sim \bar{\rho} \otimes \omega$, our diagram becomes:

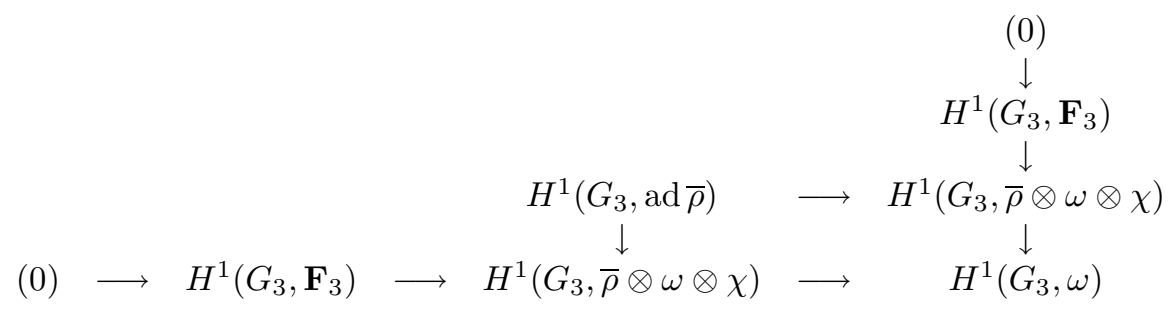

Fix a basis of $\mathbf{F}_{3}^{2}$ so that $\bar{\rho}$ takes the form

$$
\left(\begin{array}{cc}
\omega \otimes \chi & * \\
0 & \chi
\end{array}\right)
$$

Then any extension of $\bar{\rho}$ by $\bar{\rho}$ in characteristic 3 may be represented by a matrix

$$
\left(\begin{array}{cc}
\bar{\rho} & \phi \bar{\rho} \\
0 & \bar{\rho}
\end{array}\right)
$$

where the cocycle

$$
\phi=\left(\begin{array}{ll}
\phi_{11} & \phi_{12} \\
\phi_{21} & \phi_{22}
\end{array}\right) \in Z^{1}\left(G_{3}, \operatorname{ad} \bar{\rho}\right)
$$

represents the class of this extension in $\operatorname{Ext}_{\mathbf{F}_{3}\left[G_{3}\right]}^{1}(\bar{\rho}, \bar{\rho}) \cong H^{1}\left(G_{3}\right.$, ad $\left.\bar{\rho}\right)$. Moreover

- $\theta_{0}([\phi])=\left[\phi_{21}\right] \in H^{1}\left(G_{3}, \omega\right)$,

- if $\phi_{21}=0$, then $\theta_{1}([\phi])=\left[\phi_{22}\right] \in H^{1}\left(G_{3}, \mathbf{F}_{3}\right)$ and $\theta_{\omega}([\phi])=\left[\phi_{11}\right] \in H^{1}\left(G_{3}, \mathbf{F}_{3}\right)$,

- and $[\phi] \in H^{1}\left(G_{3}, \operatorname{ad}^{0} \bar{\rho}\right)$ if and only if $0=\left[\phi_{11}+\phi_{22}\right] \in H^{1}\left(G_{3}, \mathbf{F}_{3}\right)$.

In particular we have $\theta_{1}=-\theta_{\omega}$ on $H^{1}\left(G_{3}, \operatorname{ad}^{0} \bar{\rho}\right) \cap \operatorname{ker} \theta_{0}$.

We have an exact sequence

$$
(0) \longrightarrow \bar{\rho} \otimes \chi \longrightarrow \operatorname{ad}^{0} \bar{\rho} \longrightarrow \omega \longrightarrow(0),
$$

where the first map sends

$$
\left(\begin{array}{l}
x \\
y
\end{array}\right) \longmapsto\left(\begin{array}{cc}
-y / 2 & x \\
0 & y / 2
\end{array}\right)
$$

and the second map sends

$$
\left(\begin{array}{cc}
a & b \\
c & -a
\end{array}\right) \longmapsto c
$$

Thus we get an exact sequence

$$
(0) \longrightarrow H^{1}\left(G_{3}, \bar{\rho} \otimes \chi\right) \longrightarrow H^{1}\left(G_{3}, \operatorname{ad}^{0} \bar{\rho}\right) \stackrel{\theta_{0}}{\longrightarrow} H^{1}\left(G_{3}, \omega\right)
$$

and so we may identify $H^{1}\left(G_{3}\right.$, ad $\left.^{0} \bar{\rho}\right) \cap \operatorname{ker} \theta_{0}$ with $H^{1}\left(G_{3}, \bar{\rho} \otimes \chi\right)$. We also have an exact sequence

$$
(0) \longrightarrow \omega \longrightarrow \bar{\rho} \otimes \chi \longrightarrow 1 \longrightarrow(0),
$$

which gives rise to an exact sequence

$$
(0) \longrightarrow \mathbf{F}_{3} \longrightarrow H^{1}\left(G_{3}, \omega\right) \longrightarrow H^{1}\left(G_{3}, \bar{\rho} \otimes \chi\right) \longrightarrow H^{1}\left(G_{3}, \mathbf{F}_{3}\right) \longrightarrow H^{2}\left(G_{3}, \omega\right) \text {. }
$$

If we identify $H^{1}\left(G_{3}, \bar{\rho} \otimes \chi\right)$ with $H^{1}\left(G_{3}, \operatorname{ad}^{0} \bar{\rho}\right) \cap \operatorname{ker} \theta_{0}$, then the latter map $H^{1}\left(G_{3}, \bar{\rho} \otimes \chi\right) \rightarrow H^{1}\left(G_{3}, \mathbf{F}_{3}\right)$ is identified with $\theta_{\omega}=-\theta_{1}$. 
Lemma 4.7.1. The sequence

$$
(0) \longrightarrow \mathbf{F}_{3} \longrightarrow H^{1}\left(G_{3}, \omega\right) \longrightarrow H^{1}\left(G_{3}, \bar{\rho} \otimes \chi\right) \longrightarrow H^{1}\left(I_{3}, \mathbf{F}_{3}\right)
$$

is exact.

Proof. The key point is that $\bar{\rho}$ is très ramifié (compare with Proposition 6.1 of Di1]). It suffices to show that the composite

$$
H^{1}\left(G_{\mathbf{F}_{3}}, \mathbf{F}_{3}\right) \longrightarrow H^{1}\left(G_{3}, \mathbf{F}_{3}\right) \longrightarrow H^{2}\left(G_{3}, \omega\right)
$$

is injective. Suppose that $x \in H^{1}\left(G_{3}, \mathbf{F}_{3}\right)$ maps to zero in $H^{2}\left(G_{3}, \omega\right)$; then by Tate duality $x$ is annihilated by the image of the map $H^{0}\left(G_{3}, \mathbf{F}_{3}\right) \rightarrow H^{1}\left(G_{3}, \omega\right)$ coming from the short exact sequence

$$
(0) \longrightarrow \omega \longrightarrow(\bar{\rho} \otimes \chi)^{\vee} \otimes \omega \longrightarrow 1 \longrightarrow(0)
$$

Cartier dual to (4.7.1). As $(\bar{\rho} \otimes \chi)^{\vee} \otimes \omega$ is très ramifié we see that the image of

$$
H^{0}\left(G_{3}, \mathbf{F}_{3}\right) \longrightarrow H^{1}\left(G_{3}, \omega\right) \cong \mathbf{Q}_{3}^{\times} /\left(\mathbf{Q}_{3}^{\times}\right)^{3}
$$

is not contained in $\mathbf{Z}_{3}^{\times} /\left(\mathbf{Z}_{3}^{\times}\right)^{3}$. Thus

$$
x \in \operatorname{Hom}\left(\mathbf{Q}_{3}^{\times} / \mathbf{Z}_{3}^{\times}, \mathbf{F}_{3}\right) \cong H^{1}\left(G_{\mathbf{F}_{3}}, \mathbf{F}_{3}\right) \subset H^{1}\left(G_{3}, \mathbf{F}_{3}\right) \cong \operatorname{Hom}\left(\mathbf{Q}_{3}^{\times}, \mathbf{F}_{3}\right)
$$

must be zero (see Proposition 3 of $\S 1$ of Chapter XIV of [Se1]).

Corollary 4.7.2. The maps

$$
\bar{\theta}_{1}: H^{1}\left(G_{3}, \operatorname{ad}^{0} \bar{\rho}\right) \cap \operatorname{ker} \theta_{0} \longrightarrow H^{1}\left(I_{3}, \mathbf{F}_{3}\right)
$$

and

$$
\bar{\theta}_{\omega}: H^{1}\left(G_{3}, \operatorname{ad}^{0} \bar{\rho}\right) \cap \operatorname{ker} \theta_{0} \longrightarrow H^{1}\left(I_{3}, \mathbf{F}_{3}\right)
$$

have the same kernel and this has dimension 1 over $\mathbf{F}_{3}$.

Theorems 4.4.1, 4.5.1 and 4.6.2 now follow from the following results, which we will prove later. One advantage of these new formulations is that, with one exception, they refer only to $\operatorname{Ext}_{\mathcal{S}}^{1}(\bar{\rho}, \bar{\rho})$ and make no mention of the determinant or $\operatorname{ad}^{0} \bar{\rho}$, concepts which we found tricky to translate into the language of integral models.

Theorem 4.7.3. (1) $\theta_{0}: \operatorname{Ext}_{\mathcal{S}_{ \pm 1}}^{1}(\bar{\rho}, \bar{\rho}) \rightarrow H^{1}\left(G_{3}, \omega\right)$ is the zero map.

(2) $\bar{\theta}_{\omega}: \operatorname{Ext}_{\mathcal{S}_{-1}}^{1}(\bar{\rho}, \bar{\rho}) \rightarrow H^{1}\left(I_{3}, \mathbf{F}_{3}\right)$ is the zero map.

(3) $\bar{\theta}_{\omega}: H_{\mathcal{S}_{1}}^{1}\left(G_{3}, \operatorname{ad}^{0} \bar{\rho}\right) \rightarrow H^{1}\left(I_{3}, \mathbf{F}_{3}\right)$ is the zero map.

Theorem 4.7.4. (1) $\theta_{0}: \operatorname{Ext}_{\mathcal{S}_{ \pm 3}}^{1}(\bar{\rho}, \bar{\rho}) \rightarrow H^{1}\left(G_{3}, \omega\right)$ is the zero map.

(2) $\bar{\theta}_{\omega}: \operatorname{Ext}_{\delta_{ \pm 3}}^{1}(\bar{\rho}, \bar{\rho}) \rightarrow H^{1}\left(I_{3}, \mathbf{F}_{3}\right)$ is the zero map.

Theorem 4.7.5. Suppose that $i \in \mathbf{Z} / 3 \mathbf{Z}$ and $(r, s)=(2,6),(6,10),(2,10)$ or $(6,6)$.

(1) $\theta_{0}: \operatorname{Ext}_{\mathcal{S}_{i,(r, s)}}^{1}(\bar{\rho}, \bar{\rho}) \rightarrow H^{1}\left(G_{3}, \omega\right)$ is the zero map.

(2) Either $\bar{\theta}_{\omega}: \operatorname{Ext}_{\mathcal{S}_{i,(r, s)}}^{1}(\bar{\rho}, \bar{\rho}) \rightarrow H^{1}\left(I_{3}, \mathbf{F}_{3}\right)$ or $\bar{\theta}_{1}: \operatorname{Ext}_{\mathcal{S}_{i,(r, s)}}^{1}(\bar{\rho}, \bar{\rho}) \rightarrow H^{1}\left(I_{3}, \mathbf{F}_{3}\right)$ is the zero map.

The deduction of Theorems 4.4.14.5.1 and 4.6.2 from these results is immediate. 


\section{BREUIL MOdULES}

In this section we recall some results from $\mathrm{Br} 2$ (see also the summary $\mathrm{Br} 1$ ) and give some slight extensions of them. Three of the authors apologise to the fourth for the title of this section, but they find that the term "Breuil module" is much more convenient than "filtered $\phi_{1}$-module".

Throughout this section, $\ell$ will be an odd rational prime and $R$ will be a complete discrete valuation ring with fraction field $F^{\prime}$ of characteristic zero and perfect residue field $k$ of characteristic $\ell$.

5.1. Basic properties of Breuil modules. We will fix a choice of uniformiser $\pi$ of $R$ and let

$$
E_{\pi}(u)=u^{e}-\ell G_{\pi}(u)
$$

be the Eisenstein polynomial which is the minimal polynomial of $\pi$ over the fraction field of $W(k)$, so $G_{\pi}(u) \in W(k)[u]$ is a polynomial with unit constant term $G_{\pi}(0) \in$ $W(k)^{\times}$(and degree at most $e-1$ ). The $\ell^{t h}$ power map on $k[u] / u^{e \ell}$ is denoted $\phi$, and we define

$$
c_{\pi}=-\phi\left(G_{\pi}(u)\right) \in\left(k[u] / u^{e \ell}\right)^{\times} .
$$

It is very important to keep in mind that these definitions, as well as many of the definitions below, depend on the choice of the uniformiser $\pi$.

The category of $\ell$-torsion Breuil modules (or " $\ell$-torsion Breuil modules over $R$ ", or simply "Breuil modules" or "Breuil modules over $R$ ") is defined to be the category of triples $\left(\mathcal{M}, \mathcal{M}_{1}, \phi_{1}\right)$, where

- $\mathcal{M}$ is a finite free $k[u] / u^{e \ell}$-module,

- $\mathcal{M}_{1}$ is a $k[u] / u^{e \ell}$-submodule of $\mathcal{M}$ containing $u^{e} \mathcal{N}$,

- $\phi_{1}: \mathcal{M}_{1} \rightarrow \mathcal{M}$ is $\phi$-semi-linear and has image whose $k[u] / u^{e \ell}$-span is all of $\mathcal{M}$.

(A morphism $\left(\mathcal{M}, \mathcal{M}_{1}, \phi_{1}\right) \rightarrow\left(\mathcal{N}, \mathcal{N}_{1}, \psi_{1}\right)$ is a morphism $f: \mathcal{M} \rightarrow \mathcal{N}$ of $k[u] / u^{e \ell_{-}}$ modules such that $f \mathcal{M}_{1} \subset \mathcal{N}_{1}$ and $\psi_{1} \circ f=f \circ \phi_{1}$ on $\mathcal{M}_{1}$.) We define the rank of $\left(\mathcal{M}, \mathcal{M}_{1}, \phi_{1}\right)$ to be the rank of $\mathcal{M}$ over $k[u] / u^{e \ell}$. Breuil modules form an additive category (not abelian in general) in the obvious manner and this category does not

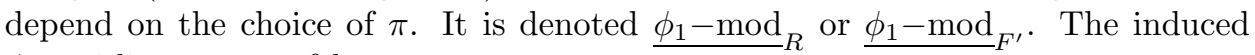
$\phi$-semi-linear map of $k$-vector spaces

$$
\bar{\phi}_{1}: \mathcal{M}_{1} / u \mathcal{M}_{1} \longrightarrow \mathcal{M} / u \mathcal{N}
$$

is bijective (because it is onto and $\# \mathcal{M}_{1} / u \mathcal{M}_{1}=\# \mathcal{M}_{1}[u] \leq \# \mathcal{M}[u]=\# \mathcal{M} / u \mathcal{M}$ ). In particular, a map of Breuil modules

$$
\left(\mathcal{M}, \mathcal{M}_{1}, \phi_{1}\right) \longrightarrow\left(\mathcal{M}^{\prime}, \mathcal{M}_{1}^{\prime}, \phi_{1}^{\prime}\right)
$$

is an isomorphism if and only if the map $\mathcal{N} \rightarrow \mathcal{M}^{\prime}$ on underlying $k[u] / u^{e \ell}$-modules is an isomorphism.

Lemma 5.1.1. Suppose that

$$
0 \longrightarrow \mathcal{M}^{\prime} \longrightarrow \mathcal{M} \longrightarrow \mathcal{M}^{\prime \prime} \longrightarrow 0
$$

is a complex of Breuil modules. The following are equivalent.

(1) The underlying sequence of $k[u] / u^{e \ell}$-modules is exact.

(2) The underlying sequence of $k[u] / u^{e \ell}$-modules is exact as is the sequence

$$
0 \longrightarrow \mathcal{M}_{1}^{\prime} \longrightarrow \mathcal{M}_{1} \longrightarrow \mathcal{M}_{1}^{\prime \prime} \longrightarrow 0
$$


(3) The complex of vector spaces

$$
0 \longrightarrow \mathcal{N}^{\prime} / u \longrightarrow \mathcal{M} / u \longrightarrow \mathcal{M}^{\prime \prime} / u \longrightarrow 0
$$

is exact.

Proof. The second statement clearly implies the first. The first implies the third as Breuil modules are free over $k[u] / u^{e \ell}$. It remains to show that the third condition implies the second. Using Nakayama's lemma and the freeness of Breuil modules we see that

$$
0 \longrightarrow \mathcal{M}^{\prime} \longrightarrow \mathcal{M} \longrightarrow \mathcal{M}^{\prime \prime} \longrightarrow 0
$$

is an exact sequence of $k[u] / u^{e \ell}$-modules. Using the bijectivity of $\bar{\phi}_{1}$, we see that the natural map

$$
f_{1}: \mathcal{M}_{1} \longrightarrow \mathcal{M}_{1}^{\prime \prime}
$$

is surjective modulo $u$ and therefore is surjective. It remains to check that the inclusion of $k[u] / u^{e \ell}$-modules $\mathcal{M}_{1}^{\prime} \subseteq \operatorname{ker}\left(f_{1}\right)$ is an equality. Since $f_{1}$ is compatible with $f: \mathcal{M} \rightarrow \mathcal{M}^{\prime \prime}$ via the inclusions $\mathcal{M}_{1} \subseteq \mathcal{M}, \mathcal{M}_{1}^{\prime \prime} \subseteq \mathcal{M}^{\prime \prime}$ and also via the maps $\phi_{1}$ and $\phi_{1}^{\prime \prime}$, it is obvious that $\operatorname{ker}\left(f_{1}\right) \subseteq \operatorname{ker}(f)=\mathcal{M}^{\prime}$ and that $\phi_{1}\left(\operatorname{ker}\left(f_{1}\right)\right) \subseteq \mathcal{M}^{\prime}$. Since $\operatorname{ker}\left(f_{1}\right)$ contains $\mathcal{M}_{1}^{\prime}$, which in turn contains $u^{e} \mathcal{M}^{\prime}$, we see that $\left(\mathcal{M}^{\prime}, \operatorname{ker}\left(f_{1}\right), \phi_{1}\right)$ is a Breuil module! Then $\left(\mathcal{M}^{\prime}, \mathcal{M}_{1}^{\prime}, \phi_{1}^{\prime}\right) \rightarrow\left(\mathcal{M}^{\prime}, \operatorname{ker}\left(f_{1}\right), \phi_{1}\right)$ defined via the identity map on $\mathcal{M}^{\prime}$ is a map of Breuil modules which is an isomorphism on underlying $k[u] / u^{e \ell}$-modules, so it must be an isomorphism of Breuil modules. This forces $\operatorname{ker}\left(f_{1}\right)=\mathcal{M}_{1}^{\prime}$.

When the equivalent conditions of this lemma are met we call the sequence of Breuil modules

$$
0 \longrightarrow \mathcal{M}^{\prime} \longrightarrow \mathcal{M} \longrightarrow \mathcal{M}^{\prime \prime} \longrightarrow 0
$$

exact.

For any Breuil module $\left(\mathcal{M}, \mathcal{M}_{1}, \phi_{1}\right)$, we define the Frobenius endomorphism $\phi$ : $\mathcal{N} \rightarrow \mathcal{M}$ by

$$
\phi(m)=\frac{1}{c_{\pi}} \phi_{1}\left(u^{e} m\right),
$$

where $c_{\pi}$ is defined as in (5.1.1). Note that this depends on our choice of uniformiser.

We let $N: W(k)[[u]] \rightarrow W(k)[[u]]$ denote the unique continuous $W(k)$-linear derivation satisfying $N u=u$, i.e. $N=u \frac{d}{d u}$. This operator "extends" to any Breuil module. More precisely, we have the following lemma.

Lemma 5.1.2. Let $\mathcal{M}$ be an object of ${\underline{\phi_{1}-\bmod }}_{R}$. There is a unique additive operator $N: \mathcal{M} \rightarrow \mathcal{M}$ (the monodromy operator) satisfying the following three conditions:

(1) $N(s x)=N(s) x+s N(x), s \in k[u] / u^{e \ell}, x \in \mathcal{M}$,

(2) $N \circ \phi_{1}=\phi \circ N$ on $\mathcal{M}_{1}$,

(3) $N(\mathcal{M}) \subset u \mathcal{M}$.

Moreover, any morphism of Breuil modules $\mathcal{M} \rightarrow \mathcal{M}^{\prime}$ automatically commutes with $N$.

Proof. Let's start with unicity. Recall we have an isomorphism $k[u] / u^{e \ell} \otimes_{k\left[u^{\ell}\right] / u^{e \ell}}$ $\phi_{1}\left(\mathcal{M}_{1}\right) \stackrel{\sim}{\rightarrow} \mathcal{M}([\overline{\mathrm{Br} 2}], 2.1 .2 .1)$. Suppose there are two operators $N$ and $N^{\prime}$ satisfying 
(1), (2) and (3) above, so $\Delta=N-N^{\prime}$ is $k[u] / u^{e \ell}$-linear and satisfies $\Delta \phi_{1}=\phi \Delta$ and $\Delta(\mathcal{M}) \subset u \mathcal{M}$. Thus,

$$
\Delta \phi_{1}\left(\mathcal{M}_{1}\right)=\phi \Delta\left(\mathcal{M}_{1}\right) \subset \phi(u \mathcal{N}) \subset u^{\ell} \mathcal{N},
$$

so $\Delta(\mathcal{N})=\Delta\left(k[u] / u^{e \ell} \otimes_{k\left[u^{\ell}\right] / u^{e \ell}} \phi_{1}\left(\mathcal{M}_{1}\right)\right) \subset u^{\ell \mathcal{M}}$. Iterating $\Delta \phi_{1}\left(\mathcal{M}_{1}\right) \subset \phi \Delta(\mathcal{M}) \subset$ $u^{\ell^{2} \mathcal{M}}$ so $\Delta(\mathcal{M}) \subset u^{\ell^{2} \mathcal{M}}$, and so on. As $u^{e \ell}=0$, we get $\Delta=0$. For the existence, let $N_{0}=N \otimes 1$ on

$$
k[u] / u^{e \ell} \otimes_{k\left[u^{\ell}\right] / u^{e \ell}} \phi_{1}\left(\mathcal{M}_{1}\right) \simeq \mathcal{M},
$$

and note $N_{0}$ satisfies $N_{0}(s x)=N(s) x+s N_{0}(x)$. Call a derivation of $\mathcal{M}$ any additive operator satisfying this relation and define successive derivations of $\mathcal{M}$ by the formula

$$
N_{j+1}\left(s \otimes \phi_{1}(x)\right)=N(s) \otimes \phi_{1}(x)+s \phi\left(N_{j}(x)\right),
$$

for $j \geq 0$. Note that $N_{j+1}$ is well defined by the following observations.

- $N\left(u^{\ell} s\right)=u^{\ell} N(s)$ and $N_{j}(u x)=u x+u N_{j}(x)$ imply that $N_{j+1}\left(u^{\ell} s \otimes \phi_{1}(x)\right)=$ $N_{j+1}\left(s \otimes \phi_{1}(u x)\right)$.

- If $\phi_{1}(x)=0$, then $x \in u^{e} \mathcal{M}$ (see (1) of Lemma 2.1.2.1 of [Br1]) and so $N_{j}(x) \in u^{e} \mathcal{M}$ and $\phi\left(N_{j}(x)\right)=0$.

As $N_{0}(\mathcal{M}) \subset u \mathcal{M}$, we have $\left(N_{j+1}-N_{j}\right)(\mathcal{M}) \subset u^{\ell^{j+1}} \mathcal{M}$, so $N_{j}=N_{j+1}$ for $j \gg 0$. This $N_{j}$ satisfies (1), (2) and (3).

The reason for introducing Breuil modules (and putting the factor $c_{\pi}^{-1}$ in the definition of $\phi$ ) is the following theorem.

Theorem 5.1.3. (1) Given the choice of uniformiser $\pi$ for $R$ there is a contravariant functor $\mathcal{M}_{\pi}$ from finite flat $R$-group schemes which are killed by $\ell$ to ${\underline{\phi_{1}-\bmod _{R}}}_{R}$ and a quasi-inverse functor $\mathcal{G}_{\pi}$.

(2) If $\overline{\mathcal{G}}$ is a finite flat $R$-group scheme killed by $\ell$, then $\mathcal{G}$ has rank $\ell^{r}$ if and only if $\mathcal{M}_{\pi}(\mathcal{G})$ has rank $r$.

(3) If $\mathcal{G}$ is a finite flat $R$-group scheme killed by $\ell$, then there is a canonical $k$-linear isomorphism

$$
D(\mathcal{G}) \otimes_{k, \mathrm{Frob}_{\ell}} k \cong \mathcal{M}_{\pi}(\mathcal{G}) / u \mathcal{M}_{\pi}(\mathcal{G}) .
$$

Under this identification, $\mathbf{F} \otimes$ Frob $_{\ell}$ corresponds to $\phi$ and $\mathbf{V} \otimes$ Frob $_{\ell}^{-1}$ corresponds to the composite

$$
\mathbf{V}_{\mathcal{M}}: \mathcal{M} / u \mathcal{N} \stackrel{\bar{\phi}_{1}^{-1}}{\longrightarrow} \mathcal{M}_{1} / u \mathcal{M}_{1} \longrightarrow \mathcal{M} / u \mathcal{M}
$$

(4) If

$$
0 \longrightarrow \mathcal{G}^{\prime} \longrightarrow \mathcal{G} \longrightarrow \mathcal{G}^{\prime \prime} \longrightarrow 0
$$

is a diagram of finite flat group schemes over $R$ which are killed by $\ell$ and if

$$
0 \longrightarrow \mathcal{M}_{\pi}\left(\mathcal{G}^{\prime \prime}\right) \longrightarrow \mathcal{M}_{\pi}(\mathcal{G}) \longrightarrow \mathcal{M}_{\pi}\left(\mathcal{G}^{\prime}\right) \longrightarrow 0
$$

is the corresponding diagram of Breuil modules, then the diagram of finite flat group schemes is a short exact sequence if and only if the diagram of Breuil modules is a short exact sequence. 
Proof. See §2.1.1, Proposition 2.1.2.2, Theorem 3.3.7, Theorem 4.2.1.6 and the proof of Theorem 3.3.5 of $\mathrm{Br2}$. In 3.3.5 of $\mathrm{Br2}$ it is shown that $\mathcal{M}_{\pi}(\mathcal{G}) / u \mathcal{M}_{\pi}(\mathcal{G})$ can be $k$-linearly identified with the crystalline Dieudonné module of $\mathcal{G} \times k$. In 4.2.14 of [BBM] the crystalline Dieudonné module of $\mathcal{G} \times k$ is identified with $D(\mathcal{G}) \otimes_{k, \text { Frob }_{\ell}} k$. The equivalence of the two notions of exactness can be deduced from the compatibility of $\mathcal{M}_{\pi}$ with Dieudonné theory, from Lemma 5.1.1 and from the fact that a complex of finite flat group schemes over $R$ is exact if and only if its special fibre is exact (see for example Proposition 1.1 of $[\mathrm{deJ}]$ ).

5.2. Examples. For $0 \leq r \leq e$ an integer and for $a \in k^{\times}$, define a Breuil module $\mathcal{M}(r, a)$ by

- $\mathcal{M}(r, a)=\left(k[u] / u^{e \ell}\right) \mathbf{e}$,

- $\mathcal{M}(r, a)_{1}=\left(k[u] / u^{e \ell}\right) u^{r} \mathbf{e}$,

- $\phi_{1}\left(u^{r} \mathbf{e}\right)=a \mathbf{e}$.

It is easy to check that $\phi_{1}$ is well defined (and uniquely determined by the given conditions). We will refer to e as the standard generator of $\mathcal{M}(r, a)$ and write $\mathcal{G}(r, a)$ for $\mathcal{G}_{\pi}(\mathcal{M}(r, a))$. The following lemma is easy to check.

Lemma 5.2.1. (1) Any Breuil module of rank 1 over $k[u] / u^{e \ell}$ is isomorphic to some $\mathcal{N}(r, a)$.

(2) There is a non-zero morphism $\mathcal{M}(r, a) \rightarrow \mathcal{M}\left(r^{\prime}, a^{\prime}\right)$ if and only if $r^{\prime} \geq r$, $r^{\prime} \equiv r \bmod \ell-1$ and $a / a^{\prime} \in\left(k^{\times}\right)^{\ell-1}$. All such morphisms are then of the form $\mathbf{e} \mapsto b u^{\ell\left(r^{\prime}-r\right) /(\ell-1)} \mathbf{e}^{\prime}$, where $b^{\ell-1}=a / a^{\prime}$.

(3) The modules $\mathcal{M}(r, a)$ and $\mathcal{M}\left(r^{\prime}, a^{\prime}\right)$ are isomorphic if and only if $r=r^{\prime}$ and $a / a^{\prime} \in\left(k^{\times}\right)^{\ell-1}$, or equivalently if and only if there are non-zero morphisms $\mathcal{M}(r, a) \rightarrow \mathcal{M}\left(r^{\prime}, a^{\prime}\right)$ and $\mathcal{M}\left(r^{\prime}, a^{\prime}\right) \rightarrow \mathcal{M N}(r, a)$.

(4) If we order the $\mathcal{N}(r, a)$ by setting $\mathcal{M}(r, a) \geq \mathcal{M}\left(r^{\prime}, a^{\prime}\right)$ if there is a nonzero morphism $\mathcal{M}\left(r^{\prime}, a^{\prime}\right) \rightarrow \mathcal{M}(r, a)$, then the set of isomorphism classes of $\mathcal{M}_{\pi}(\mathcal{G})$ 's as $\mathcal{G}$ runs over models of a fixed finite flat $F^{\prime}$-group scheme $G$ of order $\ell$ is well ordered.

(5) On $\mathcal{M}(r, a)$ we have $N \mathbf{e}=0$, so $N \circ \phi_{1}=0$.

(6) $\mathcal{G}(r, a)$ is étale (resp. multiplicative) if and only if $r=e($ resp. $r=0)$.

(7) $\mathcal{G}(0,1) \cong \mu_{\ell}$ and $\mathcal{G}\left(e,-G_{\pi}(0)\right) \cong \mathbf{Z} / \ell \mathbf{Z}$.

(8) The Cartier dual of $\mathcal{G}\left(r,-G_{\pi}(0)\right)$ is $\mathcal{G}(e-r, 1)$.

Proof. The first three parts are easy computations. For the fourth part note that two finite flat group schemes $\mathcal{G}$ and $\mathcal{G}^{\prime}$ of order $\ell$ over $R$ have isomorphic generic fibres if and only if there is a non-zero morphism $\mathcal{G} \rightarrow \mathcal{G}^{\prime}$ or $\mathcal{G}^{\prime} \rightarrow \mathcal{G}$. The fifth part is another easy computation and the sixth part follows on computing the Dieudonné module using Theorem 5.1.3.

By 3.1.2 of $[\mathrm{Br} 2]$ we see that the affine $R$-algebra of the group scheme attached to $\mathcal{M}(r, a)$ is

$$
R[X] /\left(X^{\ell}+\frac{\pi^{e-r} \widetilde{a}}{G_{\pi}(\pi)} X\right),
$$

where $\widetilde{a}$ is a lift of $a$ to $W(k)$. This has constant generic fibre if and only if $-\pi^{e-r} \widetilde{a} / G_{\pi}(\pi) \in F^{\prime}$ is an $(\ell-1)^{t h}$ power. This occurs if and only if $r \equiv e \bmod \ell-1$ and $-a / G_{\pi}(0) \in k$ is an $(\ell-1)^{t h}$ power. Thus $\mathcal{M}\left(e,-G_{\pi}(0)\right)$ corresponds to the étale group scheme $\mathbf{Z} / \ell \mathbf{Z}$ over $R$. 
Next, we show that the group scheme $\mathcal{G}$ corresponding to the Breuil module $\mathcal{M}(0,1)$ is isomorphic to $\mu_{\ell}$. By using the relation between Breuil modules and Dieudonné modules (see Theorem [5.1.3) we see that the Dieudonné module of the closed fibre of $\mathcal{G}$ is isomorphic to the Dieudonné module of the closed fibre of $\mu_{\ell}$. This forces $\mathcal{G} \stackrel{\sim}{\rightarrow} \mu_{\ell}$, since we may consider Cartier duals and observe that a finite flat $R$-group scheme $\mathcal{G}$ is étale if and only if its special fibre is étale, and then $\S 18.5 .15$ of book $\mathrm{IV}_{4}$ of [EGA] may be used.

This establishes the seventh part. The final part follows from parts four and seven.

Now suppose that $0 \leq r, s \leq e$ are integers and choose $a, b \in k^{\times}$and $f \in$ $u^{\max (0, r+s-e)} k[u] / u^{e \ell}$. We can define an extension class

$$
(0) \longrightarrow \mathcal{M}(s, b) \longrightarrow \mathcal{M}(s, b ; r, a ; f) \longrightarrow \mathcal{M}(r, a) \longrightarrow(0)
$$

in $\underline{\phi 1}^{-\bmod } R$ by

- $\mathcal{M}(s, b ; r, a ; f)=\left(k[u] / u^{e \ell}\right) \mathbf{e} \oplus\left(k[u] / u^{e \ell}\right) \mathbf{e}^{\prime}$,

- $\mathcal{M}(s, b ; r, a ; f)_{1}=\left\langle u^{s} \mathbf{e}, u^{r} \mathbf{e}^{\prime}+f \mathbf{e}\right\rangle$,

- $\phi_{1}\left(u^{s} \mathbf{e}\right)=b \mathbf{e}$ and $\phi_{1}\left(u^{r} \mathbf{e}^{\prime}+f \mathbf{e}\right)=a \mathbf{e}^{\prime}$,

- the standard generator of $\mathcal{M}(s, b)$ maps to e,

- e maps to 0 and $\mathbf{e}^{\prime}$ maps to the standard generator in $\mathcal{M}(r, a)$.

The following lemma is also easy to check.

Lemma 5.2.2. (1) Any extension of $\mathcal{M}(r, a)$ by $\mathcal{M}(s, b)$ in $\underline{\phi}_{1}-\bmod _{R}$ is isomorphic to $\mathcal{M}(s, b ; r, a ; f)$ for some $f \in u^{\max (0, r+s-e)} k[u] / u^{e \ell}$.

(2) Two such extensions $\mathcal{M}(s, b ; r, a ; f)$ and $\mathcal{M}\left(s, b ; r, a ; f^{\prime}\right)$ are isomorphic as extension classes if and only if

$$
f^{\prime}-f=u^{s} h-(b / a) u^{r} h^{\ell}
$$

for some $h \in k[u] / u^{e \ell}$, in which case one such isomorphism fixes e and sends $\mathbf{e}^{\prime}$ to $\mathbf{e}^{\prime}-(b / a) h^{\ell} \mathbf{e}$.

We remark that $f \in u^{\max (0, r+s-e)} k[u] / u^{e \ell}$ is required so that $\mathcal{M}(s, b ; r, a ; f)_{1} \supset$ $u^{e} \mathcal{M}(s, b ; r, a ; f)$. We will write $\mathcal{G}(s, b ; r, a ; f)$ for $\mathcal{G}_{\pi}(\mathcal{M}(s, b ; r, a ; f))$.

We will also need some slight extensions of these results to allow for coefficients. To this end let $k^{\prime} / \mathbf{F}_{\ell}$ be a finite extension linearly disjoint from $k$ and write $k^{\prime} k$ for the field $k^{\prime} \otimes_{\mathbf{F}_{\ell}} k$. For $0 \leq r \leq e$ an integer and for $a \in\left(k^{\prime} k\right)^{\times}$, define a Breuil module, $\mathcal{M}\left(k^{\prime} ; r, a\right)$, with an action of $k^{\prime}$ by

- $\mathcal{M}\left(k^{\prime} ; r, a\right)=\left(\left(k^{\prime} k\right)[u] / u^{e \ell}\right) \mathbf{e}$,

- $\mathcal{M}\left(k^{\prime} ; r, a\right)_{1}=\left(\left(k^{\prime} k\right)[u] / u^{e \ell}\right) u^{r} \mathbf{e}$,

- $\phi_{1}\left(u^{r} \mathbf{e}\right)=a \mathbf{e}$.

We will let $\phi$ denote the automorphism of $k^{\prime} k[u]$, which is the identity on $k^{\prime}$ and which raises elements of $k[u]$ to the $\ell^{t h}$ power. The following lemma is easy to check.

Lemma 5.2.3. (1) Any Breuil module with an action of $k^{\prime}$ which is free of rank $\left[k^{\prime}: k\right]$ over $k[u] / u^{e \ell}$ is isomorphic to some $\mathcal{M}\left(k^{\prime} ; r, a\right)$.

(2) There is a non-zero morphism $\mathcal{M}\left(k^{\prime} ; r, a\right) \rightarrow \mathcal{M}\left(k^{\prime} ; r^{\prime}, a^{\prime}\right)$ if and only if $r^{\prime} \geq r$, $r^{\prime} \equiv r \bmod \ell-1$ and $a / a^{\prime} \in \phi(b) / b$ for some $b \in\left(k^{\prime} k\right)^{\times}$. All such morphisms are then of the form $\mathbf{e} \mapsto b^{\prime} u^{\ell\left(r^{\prime}-r\right) /(\ell-1)} \mathbf{e}^{\prime}$, where $b \in\left(k^{\prime} k\right)^{\times}$and $\phi\left(b^{\prime}\right) / b^{\prime}=$ $a / a^{\prime}$. 
(3) The modules $\mathcal{M}\left(k^{\prime} ; r, a\right)$ and $\mathcal{M}\left(k^{\prime} ; r^{\prime}, a^{\prime}\right)$ are isomorphic if and only if $r=r^{\prime}$ and $a / a^{\prime} \in\left(\left(k^{\prime} k\right)^{\times}\right)^{\phi-1}$.

(4) On $\mathcal{M}\left(k^{\prime} ; r, a\right)$ we have $N \mathbf{e}=0$ and so $N \circ \phi_{1}=0$.

(5) $\mathcal{G}_{\pi}\left(\mathcal{M}\left(k^{\prime} ; r, a\right)\right)$ is étale (resp. multiplicative) if and only if $r=e$ (resp. $r=0)$.

Now choose $0 \leq r, s \leq e$ integers, $a, b \in\left(k^{\prime} k\right)^{\times}$and $f \in u^{\max (0, r+s-e)}\left(k^{\prime} k\right)[u] / u^{e \ell}$. We define an extension class

$$
(0) \longrightarrow \mathcal{M}\left(k^{\prime} ; s, b\right) \longrightarrow \mathcal{M}\left(k^{\prime} ; s, b ; r, a ; f\right) \longrightarrow \mathcal{M}\left(k^{\prime} ; r, a\right) \longrightarrow(0)
$$

in $\underline{\phi_{1}-\bmod }{ }_{R}$ with an action of $k^{\prime}$ by

- $\mathcal{M}\left(k^{\prime} ; s, b ; r, a ; f\right)=\left(\left(k^{\prime} k\right)[u] / u^{e \ell}\right) \mathbf{e} \oplus\left(\left(k^{\prime} k\right)[u] / u^{e \ell}\right) \mathbf{e}^{\prime}$,

- $\mathcal{M}\left(k^{\prime} ; s, b ; r, a ; f\right)_{1}=\left\langle u^{s} \mathbf{e}, u^{r} \mathbf{e}^{\prime}+f \mathbf{e}\right\rangle$,

- $\phi_{1}\left(u^{s} \mathbf{e}\right)=b \mathbf{e}$ and $\phi_{1}\left(u^{r} \mathbf{e}^{\prime}+f \mathbf{e}\right)=a \mathbf{e}^{\prime}$,

- the standard generator of $\mathcal{M}\left(k^{\prime} ; s, b\right)$ maps to $\mathbf{e}$,

- e maps to 0 and $\mathbf{e}^{\prime}$ to the standard generators in $\mathcal{M}\left(k^{\prime} ; r, a\right)$.

Then the following lemma is easy to check.

Lemma 5.2.4. (1) Any extension of $\mathcal{M}\left(k^{\prime} ; r, a\right)$ by $\mathcal{M}\left(k^{\prime} ; s, b\right)$ in $\underline{\phi}_{1}-\bmod { }_{R}$ with

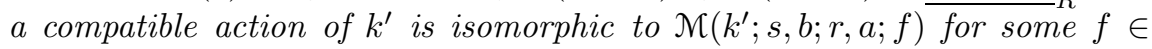
$u^{\max (0, r+s-e)}\left(k^{\prime} k\right)[u] / u^{e \ell}$.

(2) Two such extensions $\mathcal{M}\left(k^{\prime} ; s, b ; r, a ; f\right)$ and $\mathcal{M}\left(k^{\prime} ; s, b ; r, a ; f^{\prime}\right)$ are isomorphic (as extensions) if and only if

$$
f^{\prime}-f=u^{s} h-(b / a) u^{r} \phi(h)
$$

for some $h \in\left(k^{\prime} k\right)[u] / u^{e \ell}$, in which case one such isomorphism fixes $\mathbf{e}$ and sends $\mathbf{e}^{\prime}$ to $\mathbf{e}^{\prime}-(b / a) \phi(h) \mathbf{e}$.

We will write $\mathcal{G}\left(k^{\prime} ; r, a ; s, b ; f\right)$ and $\mathcal{G}\left(k^{\prime} ; r, a\right)$ for $\mathcal{G}_{\pi}\left(\mathcal{M}\left(k^{\prime} ; r, a ; s, b ; f\right)\right)$ and $\mathcal{G}_{\pi}\left(\mathcal{M}\left(k^{\prime} ; r, a\right)\right)$ respectively.

5.3. Relationship to syntomic sheaves. Let us first recall some of the notations of [Br1] and [Br2]. Let $\operatorname{Spf}(R)_{\text {syn }}$ be the small $\ell$-adic formal syntomic site over $R$, $S$ the $\ell$-adic completion of $W(k)\left[u, \frac{u^{i e}}{i !}\right]_{i \in \mathbf{N}}, S_{n}=S / \ell^{n} S, E_{n}=\operatorname{Spec}\left(S_{n}\right)$ and for any $\mathfrak{X} \in \operatorname{Spf}(R)_{\text {syn }}$ :

$$
\mathcal{O}_{n, \pi}^{\text {cris }}(\mathfrak{X})=H^{0}\left(\left(\mathfrak{X}_{n} / E_{n}\right)_{\text {cris }}, \mathcal{O}_{\mathfrak{X}_{n} / E_{n}}\right),
$$

where $\mathfrak{X}_{n}=\mathfrak{X} \times_{R} R / \ell^{n}$ is viewed over $E_{n}$ via the thickening $\left(\operatorname{Spec}\left(R / \ell^{n}\right) \hookrightarrow\right.$ $\left.E_{n}, u \mapsto \pi\right)$. It turns out $\mathcal{O}_{n, \pi}^{\text {cris }}$ is the sheaf of $S_{n}$-modules on $\operatorname{Spf}(R)_{\text {syn }}$ associated to the presheaf (cf. the proof of Lemma 2.3.2 in $\mathrm{Br} 2$ ):

$$
\begin{aligned}
\mathfrak{X} & \mapsto\left(W_{n}(k)[u] \otimes_{\phi^{n}, W_{n}(k)} W_{n}\left(\Gamma\left(\mathfrak{X}_{1}, \mathcal{O}_{\mathfrak{X}_{1}}\right)\right)\right)^{D P} \\
& =\left(\left(W_{n}(k)[u] / u^{e \ell^{n}}\right) \otimes_{\phi^{n}, W_{n}(k)} W_{n}\left(\Gamma\left(\mathfrak{X}_{1}, \mathcal{O}_{\mathfrak{X}_{1}}\right)\right)\right)^{D P} .
\end{aligned}
$$

Here, the subscript " $\phi^{n}$ " means we twist by the $n^{\text {th }}$ power of the Frobenius when sending $W_{n}(k)$ to $W_{n}(k)[u]$ and the exponent " $D P$ " means we take the divided power envelope with respect to the kernel of the canonical map:

$$
\begin{array}{ccc}
W_{n}(k)[u] \otimes_{\phi^{n}, W_{n}(k)} W_{n}\left(\Gamma\left(\mathfrak{X}_{1}, \mathcal{O}_{\mathfrak{X}_{1}}\right)\right) & \longrightarrow & \Gamma\left(\mathfrak{X}_{n}, \mathcal{O}_{\mathfrak{X}_{n}}\right) \\
s(u) \otimes\left(w_{0}, \ldots, w_{n-1}\right) & \longmapsto & s(\pi)\left(\hat{w}_{0}^{\ell^{n}}+\ell \hat{w}_{1}^{\ell^{n-1}}+\ldots+\ell^{n-1} \hat{w}_{n-1}^{\ell}\right),
\end{array}
$$


where $\hat{w}_{i}$ is a local lifting of $w_{i}$, these divided powers being required to be compatible with the usual divided powers $\gamma_{i}(\ell x)=\frac{\ell^{i}}{i !} x^{i}$ (i.e. we take the divided power envelope relative to the usual divided power structure on the maximal ideal of $W_{n}(k)$ ). Note that the latter map induces a canonical surjection of sheaves of $S_{n}$-modules on $\operatorname{Spf}(R)_{\mathrm{syn}}$ :

$$
\mathcal{O}_{n, \pi}^{\text {cris }} \rightarrow \mathcal{O}_{n},
$$

where $\mathcal{O}_{n}(\mathfrak{X})=\Gamma\left(\mathfrak{X}_{n}, \mathcal{O}_{\mathfrak{X}_{n}}\right)$. We denote by ${\underset{f}{n, \pi}}_{n, \pi}^{\text {cris }}$ the kernel of this surjection. For any $n$, let $\phi: S_{n} \rightarrow S_{n}$ be the unique lifting of Frobenius such that $\phi(u)=u^{\ell}$ and $\phi\left(\frac{u^{i e}}{i !}\right)=\frac{u^{i e \ell}}{i !}$. The sheaf $\mathcal{O}_{n, \pi}^{\text {cris }}$ is equipped with the crystalline Frobenius $\phi$, which is also induced by the map $s(u) \otimes\left(w_{0}, \ldots, w_{n-1}\right) \mapsto \phi(s(u)) \otimes\left(w_{0}^{\ell}, \ldots, w_{n-1}^{\ell}\right)$ on the above presheaf (5.3.1). (Here $\phi$ on $W_{n}(k)[u]$ is Frobenius on $W_{n}(k)$ and takes $u$ to $u^{\ell}$.) Since $\ell$ divides $\phi(x)-x^{\ell}$, we get $\phi\left(J_{n, \pi}^{c r i s}\right) \subset \ell \mathcal{O}_{n, \pi}^{\text {cris }}$ for all $n$, so we can define an $S_{1}$-linear $\phi_{1}=\left.\frac{\phi}{\ell}\right|_{\left.\right|_{n, \pi} ^{c r i s}}$ by the usual "flatness" trick (see $\S 2.3$ of [Br2]). Let $N: S_{n} \rightarrow S_{n}$ be the unique $W_{n}(k)$-linear derivation such that $N(u)=u$ and $N\left(\gamma_{i}\left(u^{e}\right)\right)=e u^{e} \gamma_{i-1}\left(u^{e}\right)=i e \gamma_{i}\left(u^{e}\right)$. Finally define:

$$
N: \mathcal{O}_{n, \pi}^{\text {cris }} \rightarrow \mathcal{O}_{n, \pi}^{\text {cris }}
$$

to be the unique $W_{n}(k)$-linear morphism of sheaves which on the presheaf (5.3.1) is given by $N\left(\gamma_{i}\left(\sum s \otimes w\right)\right)=\left(\sum N(s) \otimes w\right) \gamma_{i-1}\left(\sum s \otimes w\right)$. Note that $N \circ \phi=\ell \phi \circ N$, so $N \circ \phi_{1}=\phi \circ N$ on $\partial_{n, \pi}^{c r i s}$.

Let $\mathcal{G}$ be a finite flat group scheme over $R$, which is killed by $\ell$. Viewing $\mathcal{G}$ as a formal scheme over $R$, it is an object in $\operatorname{Spf}(R)_{\mathrm{syn}}$. Viewing it as a sheaf of groups on $\operatorname{Spf}(R)_{\mathrm{syn}}$, its associated Breuil module is defined as:

(1) $\mathcal{M}_{\pi}(\mathcal{G})=\operatorname{Hom}_{\text {sheaves of groups }}\left(\mathcal{G}, \mathcal{O}_{1, \pi}^{\text {cris }}\right) \otimes_{S_{1}} k[u] / u^{e \ell}$,

(2) $\mathcal{M}_{\pi}(\mathcal{G})_{1}=$ image of $\operatorname{Hom}_{\text {sheaves of groups }}\left(\mathcal{G}, \partial_{1, \pi}^{\text {cris }}\right) \otimes_{S_{1}} k[u] / u^{e \ell}$ in $\mathcal{M}_{\pi}(\mathcal{G})$,

(3) $\phi_{1}$ is induced by $\phi_{1} \otimes \phi$,

where the $S_{1}$-module structures are induced by the compatible $S_{1}$ actions on $\mathcal{O}_{1, \pi}^{\text {cris }}$ and $\mathcal{J}_{1, \pi}^{c r i s}$ (see $\S 3.2$ and $\S 2.1 .2 .2$ of $[\mathrm{Br} 2]$ ). Here $S_{1} \rightarrow k[u] / u^{e \ell}$ is the surjection that sends $u$ to $u, \gamma_{i}\left(u^{e}\right)$ to $\gamma_{i}\left(u^{e}\right)$ for $i<l$ and $\gamma_{i}\left(u^{e}\right)$ to 0 for $i \geq l$.

We record for future reference the following straightforward observation.

Lemma 5.3.1. If we denote by $\Delta$ (resp. $\left.\operatorname{pr}_{i}, i \in\{1,2\}\right)$ the coproduct (resp. the two projections)

$$
\mathcal{G} \times \operatorname{Spec}(R) \mathcal{G} \rightarrow \mathcal{G}
$$

then for any sheaf of commutative groups $\mathcal{F}$ on $\operatorname{Spf}(R)_{\text {syn }}$ we have:

$$
\operatorname{Hom}_{\text {sheaves of groups }}(\mathcal{G}, \mathcal{F})=\left\{x \in \mathcal{F}(\mathcal{G}) \mid\left(\Delta^{*}-\operatorname{pr}_{1}^{*}-\operatorname{pr}_{2}^{*}\right)(x)=0\right\} .
$$

The operator $N$ on $\mathcal{O}_{1, \pi}^{\text {cris }}$ induces an operator $N$ on $\operatorname{Hom}_{\text {sheaves of groups }}\left(\mathcal{G}, \mathcal{O}_{1, \pi}^{\text {cris }}\right)$, hence on $\mathcal{M}_{\pi}(\mathcal{G})$.

Lemma 5.3.2. The above operator $N$ on $\mathcal{M}_{\pi}(\mathcal{G})$ coincides with the operator $N$ defined in Lemma 5.1.2.

Proof. By unicity in Lemma 5.1.2, we only have to prove that $N$ satisfies $N\left(\mathcal{M}_{\pi}(\mathcal{G})\right.$ ) $\subset u \mathcal{M}_{\pi}(\mathcal{G})$, since the other conditions are automatically satisfied. It's enough to prove that $N\left(\phi_{1}(x)\right)=(\phi \circ N)(x) \in u \mathcal{M}_{\pi}(\mathcal{G})$ for any $x \in \mathcal{M}_{\pi}(\mathcal{G})_{1}$. But $u^{e \ell-\ell} \phi \circ N=$ 0 on $\mathcal{O}_{1, \pi}^{\text {cris }}$ because it is so on $\left(k[u] \otimes \Gamma\left(\mathfrak{X}_{1}, \mathcal{O}_{\mathfrak{X}_{1}}\right)\right)^{D P}$. Thus one also has $u^{\ell \ell-\ell} \phi \circ N=$ 
0 on $\operatorname{Hom}_{\text {groups }}\left(\mathcal{G}, \mathcal{O}_{1, \pi}^{\text {cris }}\right)$, hence on $\mathcal{M}_{\pi}(\mathcal{G})$. This implies $\phi \circ N\left(\mathcal{M}_{\pi}(\mathcal{G})\right) \subset u^{\ell} \mathcal{M}_{\pi}(\mathcal{G}) \subset$ $u \mathcal{M}_{\pi}(\mathcal{G})$ since $\mathcal{M}_{\pi}(\mathcal{G})$ is free over $k[u] / u^{e \ell}$.

5.4. Base change. In this section we will examine the relationship of the functor $\mathcal{M}_{\pi}$ with two instances of base change. First we consider unramified base change.

Let $k^{\prime}$ be a perfect field of characteristic $\ell$ which is an extension of $k$ and $R^{\prime}=$ $R \otimes_{W(k)} W\left(k^{\prime}\right)$. Choose $\pi^{\prime}=\pi \otimes 1$ as uniformiser in $R^{\prime}$. If $\mathfrak{X} \in \operatorname{Spf}(R)_{\text {syn }}$, let $\mathfrak{X}^{\prime}=\operatorname{Spf}\left(R^{\prime}\right) \times_{\operatorname{Spf}(R)} \mathfrak{X}$ and define:

$$
\mathcal{O}_{n, \pi}^{\text {cris }}(\mathfrak{X})=\mathcal{O}_{n, \pi^{\prime}}^{\text {cris }}\left(\mathfrak{X}^{\prime}\right) \text { and } \mathcal{J}_{n, \pi}^{\text {cris }}(\mathfrak{X})=\mathcal{J}_{n, \pi^{\prime}}^{\text {cris }}\left(\mathfrak{X}^{\prime}\right) \text {. }
$$

As in the proof of 2.3.2 of [Br2], we have that $\mathcal{O}_{n, \pi}^{\text {cris }}{ }^{\prime}$ is the sheaf on $\operatorname{Spf}(R)_{\mathrm{syn}}$ associated to the presheaf:

$$
\begin{aligned}
\mathfrak{X} & \mapsto\left(W_{n}\left(k^{\prime}\right)[u] \otimes_{\phi^{n}, W_{n}\left(k^{\prime}\right)} W_{n}\left(\Gamma\left(\mathfrak{X}_{1}^{\prime}, \mathcal{O}_{\mathfrak{X}_{1}^{\prime}}\right)\right)\right)^{D P} \\
& =\left(W_{n}\left(k^{\prime}\right)[u] \otimes_{\phi^{n}, W_{n}\left(k^{\prime}\right)} W_{n}\left(k^{\prime} \otimes_{k} \Gamma\left(\mathfrak{X}_{1}, \mathcal{O}_{\mathfrak{X}_{1}}\right)\right)\right)^{D P} .
\end{aligned}
$$

Define $S_{n}^{\prime}$ as $S_{n}$ but with $k^{\prime}$ instead of $k$. There is a canonical isomorphism of sheaves:

$$
\mathcal{O}_{n, \pi}^{\text {cris }} \otimes_{S_{n}} S_{n}^{\prime}=\mathcal{O}_{n, \pi}^{\text {cris }} \otimes_{W_{n}(k)} W_{n}\left(k^{\prime}\right) \stackrel{\sim}{\rightarrow} \mathcal{O}_{n, \pi}^{\text {cris }^{\prime}}
$$

coming from the obvious isomorphism:

$$
\begin{aligned}
\left(W_{n}\left(k^{\prime}\right)[u] / u^{e \ell^{n}}\right) \otimes_{\phi^{n}, W_{n}(k)} & W_{n}\left(\Gamma\left(\mathfrak{X}_{1}, \mathcal{O}_{\mathfrak{X}_{1}}\right)\right) \\
\stackrel{\sim}{\rightarrow} & \left(W_{n}\left(k^{\prime}\right)[u] / u^{e \ell^{n}}\right) \otimes_{\phi^{n}, W_{n}\left(k^{\prime}\right)} W_{n}\left(k^{\prime} \otimes_{k} \Gamma\left(\mathfrak{X}_{1}, \mathcal{O}_{\mathfrak{X}_{1}}\right)\right)
\end{aligned}
$$

and one easily sees it induces an isomorphism $\operatorname{f}_{n, \pi}^{c r i s} \otimes_{W_{n}(k)} W_{n}\left(k^{\prime}\right) \stackrel{\sim}{\rightarrow} \operatorname{f}_{n, \pi}^{c r i s^{\prime}}$. Moreover, we have the following obvious lemma.

Lemma 5.4.1. The diagram of sheaves on $\operatorname{Spf}(R)_{\mathrm{syn}}$ :

is commutative.

$$
\begin{array}{ccc}
\underset{n, \pi}{\text { cris }} \otimes_{W_{n}(k)} W_{n}\left(k^{\prime}\right) & \stackrel{\sim}{\longrightarrow} & J_{n, \pi}^{c r i s} \\
\downarrow \phi_{1} \otimes \phi & & \phi_{1} \downarrow \\
\mathcal{O}_{n, \pi}^{c r i s} \otimes_{W_{n}(k)} W_{n}\left(k^{\prime}\right) & \stackrel{\sim}{\longrightarrow} & \mathcal{O}_{n, \pi}^{c r i s^{\prime}}
\end{array}
$$

Using the identification from \$5.3, Lemma 5.3.1 and Lemma 5.4.1 (for $n=1$ ), together with obvious functorialities, we obtain after tensoring by $k[u] / u^{e \ell}$ the following corollary.

Corollary 5.4.2. Let $\mathcal{G}$ be a finite flat group scheme over $R$, which is killed by $\ell$. Let $k^{\prime} / k$ be an extension of fields with $k^{\prime}$ perfect and let $\pi^{\prime}=\pi \otimes 1$, a uniformiser for $R^{\prime}=R \otimes_{W(k)} W\left(k^{\prime}\right)$. Then there is a canonical isomorphism in the category $\underline{\phi_{1}-\bmod _{R}}$

$$
\left(\mathcal{M}_{\pi}(\mathcal{G}) \otimes_{k} k^{\prime}, \mathcal{M}_{\pi}(\mathcal{G})_{1} \otimes_{k} k^{\prime}, \phi_{1} \otimes \phi\right) \stackrel{\sim}{\rightarrow}\left(\mathcal{M}_{\pi^{\prime}}\left(\mathcal{G}^{\prime}\right), \mathcal{M}_{\pi^{\prime}}\left(\mathcal{G}^{\prime}\right)_{1}, \phi_{1}\right)
$$

compatible with composites of such residue field extensions.

We will now turn to the case of base change by a continuous automorphism $g: R \stackrel{\sim}{\rightarrow} R$. For any $s=\sum w_{i} u^{i} \in W(k)[[u]]$, let ${ }^{(g)} s=\sum g\left(w_{i}\right) u^{i}$ and ${ }^{(\phi)} s=$ $\sum \phi\left(w_{i}\right) u^{i}$, where $g$ and $\phi$ act on $W(k)$ through their action on $k$. Choose $H_{g}(u) \in$ $W(k)[[u]]$ such that $g(\pi)=\pi H_{g}(\pi)$. Notice that $H_{g}(u) \in W(k)[[u]]^{\times}$. Define $\widehat{g}: W(k)[[u]] \stackrel{\sim}{\rightarrow} W(k)[[u]]$ by $\widehat{g}\left(\sum w_{i} u^{i}\right)=\sum g\left(w_{i}\right) u^{i} H_{g}(u)^{i}$. 
Lemma 5.4.3. There is a unique element ${ }_{g} t(u) \in W(k)[[u]]$ such that, if ${ }_{g} \phi$ is defined by ${ }_{g} \phi\left(\sum w_{i} u^{i}\right)=\sum \phi\left(w_{i}\right)\left(u^{\ell}\left(1+\ell_{g} t(u)\right)\right)^{i}$, one has $\widehat{g}{ }_{g} \phi=\phi \circ \widehat{g}$.

Proof. One has to solve in $W(k)[[u]]$ :

$$
1+\ell_{g}^{(g)} t\left(u H_{g}(u)\right)=\left(H_{g}(u)^{-1}\right)^{\ell}(\phi) H_{g}\left(u^{\ell}\right)
$$

(where the two sides clearly belong to $1+\ell W(k)[[u]])$. As $H_{g}(u) \in W(k)[[u]]^{\times}$, there is a unique $K_{g} \in u W(k)[[u]]^{\times}$such that $K_{g}(u) H_{g}\left(K_{g}(u)\right)=u$, so we have

$$
1+\ell_{g}^{(g)} t(u)=\left(H_{g}\left(K_{g}(u)\right)^{-1}\right)^{\ell}(\phi) H_{g}\left(K_{g}(u)^{\ell}\right) .
$$

For any object $\mathcal{M}$ of ${\underline{\phi_{1}-\bmod }}_{R}$, define ${ }_{g} \phi_{1}: \mathcal{M}_{1} \rightarrow \mathcal{M}$ by the following formula:

$$
{ }_{g} \phi_{1}(x)=\phi_{1}(x)+{ }_{g} t(u) N\left(\phi_{1}(x)\right)
$$

where $N$ is as in Lemma 5.1 .2

For any $\mathfrak{X} \in \operatorname{Spf}(R)_{\text {syn }}$, let ${ }^{g} \mathfrak{X}=\operatorname{Spf}(R) \times_{g^{*}, \operatorname{Spf}(R)} \mathfrak{X}$ and define:

$$
\mathcal{O}_{n, \pi}^{\text {cris }},(g)(\mathfrak{X})=\mathcal{O}_{n, \pi}^{\text {cris }}\left({ }^{g} \mathfrak{X}\right) \quad \text { and } \quad \operatorname{J}_{n, \pi}^{\text {cris },(g)}(\mathfrak{X})=J_{n, \pi}^{\text {cris }}\left({ }^{g} \mathfrak{X}\right) .
$$

Then $\mathcal{O}_{n, \pi}^{\text {cris, }(g)}$ is the sheaf on $\operatorname{Spf}(R)_{\text {syn }}$ associated to the presheaf:

$$
\begin{aligned}
\mathfrak{X} & \mapsto\left(W_{n}(k)[u] \otimes_{\phi^{n}, W_{n}(k)} W_{n}\left(\Gamma\left({ }^{g} \mathfrak{X}_{1}, \mathcal{O}_{g} \mathfrak{X}_{1}\right)\right)\right)^{D P} \\
& =\left(W_{n}(k)[u] \otimes_{\phi^{n}, W_{n}(k)} W_{n}\left(R \otimes_{g, R} \Gamma\left(\mathfrak{X}_{1}, \mathcal{O}_{\mathfrak{X}_{1}}\right)\right)\right)^{D P} .
\end{aligned}
$$

Let $\widehat{g}: S_{n} \rightarrow S_{n}$ be the unique ring isomorphism such that

$$
\widehat{g}\left(w_{i} \frac{u^{e i+j}}{i !}\right)=g\left(w_{i}\right) \frac{u^{e i+j}}{i !} H_{g}(u)^{e i+j}
$$

for $0 \leq j<e, i \geq 0$. There is a canonical isomorphism of sheaves:

$$
\mathcal{O}_{n, \pi}^{\text {cris }} \otimes_{S_{n}, \hat{g}} S_{n} \stackrel{\sim}{\longrightarrow} \mathcal{O}_{n, \pi}^{\text {cris, }},(g)
$$

coming from the obvious $\widehat{g}$-semi-linear isomorphism:

$$
\begin{aligned}
&\left(W_{n}(k)[u] / u^{e \ell^{n}}\right) \otimes_{\phi^{n}, W_{n}(k)} W_{n}\left(\Gamma\left(\mathfrak{X}_{1}, \mathcal{O}_{\mathfrak{X}_{1}}\right)\right) \\
& \stackrel{\sim}{\longrightarrow}\left(W_{n}(k)[u] / u^{e \ell^{n}}\right) \otimes_{\phi^{n}, W_{n}(k)} W_{n}\left(R \otimes_{g, R} \Gamma\left(\mathfrak{X}_{1}, \mathcal{O}_{\mathfrak{X}_{1}}\right)\right) \\
& s \otimes\left(w_{0}, \ldots, w_{n-1}\right) \longmapsto \\
& \stackrel{g}{g}(s) \otimes\left(1 \otimes w_{0}, \ldots, 1 \otimes w_{n-1}\right)
\end{aligned}
$$

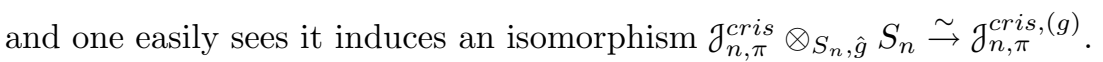

Define ${ }_{g} \phi: S_{n} \rightarrow S_{n}$ as in Lemma 5.4.3 and define:

$$
{ }_{g} \phi: \mathcal{O}_{n, \pi}^{\text {cris }} \longrightarrow \mathcal{O}_{n, \pi}^{\text {cris }}
$$

to be the unique morphism of sheaves which is induced by ${ }_{g} \phi\left(\gamma_{i}\left(\sum s \otimes w\right)\right)=$ $\gamma_{i}\left(\sum_{g} \phi(s) \otimes \phi(w)\right)$ on the presheaf (5.3.1) (see $\$ 5.3$ and note that this is well defined). Since ${ }_{g} \phi\left(\mathcal{J}_{n, \pi}^{c r i s}\right) \subset \ell \mathcal{O}_{n, \pi}^{c r i s}$, we can define ${ }_{g} \phi_{1}=\left.\frac{g \phi}{\ell}\right|_{\mathcal{J}_{n, \pi}^{c r i s}}$. 
Lemma 5.4.4. The diagram of sheaves on $\operatorname{Spf}(R)_{\mathrm{syn}}$ :

$$
\begin{aligned}
& \mathrm{J}_{n, \pi}^{\text {cris }} \otimes_{S_{n}, \hat{g}} S_{n} \stackrel{\sim}{\rightarrow} \mathrm{J}_{n, \pi}^{\text {cris }},(g) \\
& \downarrow_{g} \phi_{1} \otimes \phi \quad \phi_{1} \downarrow \\
& \mathcal{O}_{n, \pi}^{\text {cris }} \otimes_{S_{n}, \hat{g}} S_{n} \stackrel{\sim}{\rightarrow} \mathcal{O}_{n, \pi}^{\text {cris },(g)}
\end{aligned}
$$

is commutative. Moreover we have on $\mathrm{g}_{n, \pi}^{\mathrm{cris}}$ :

$$
{ }_{g} \phi_{1}=\sum_{i=0}^{\infty}\left(\frac{\log \left(1+\ell_{g} t(u)\right)}{\ell}\right)^{i} \frac{N^{i}}{i !} \circ \phi_{1},
$$

where $N$ is defined as in 5.3 .

Proof. By working modulo $\ell^{n+1}$, i.e. with $\mathcal{J}_{n+1, \pi}^{c r i s}$ and $g \phi$, and looking on the above presheaves, the proof is completely straightforward.

Let $\mathcal{G}$ be a finite flat group scheme over $R$ which is killed by $\ell$. Note that thanks to Lemma 5.3.2 and the formula for ${ }_{g} \phi_{1}$ in Lemma 5.4.4, the operator $\mathcal{M}_{\pi}(\mathcal{G})_{1} \rightarrow \mathcal{M}_{\pi}(\mathcal{G})$ induced by the map ${ }_{g} \phi_{1}: \mathcal{J}_{n, \pi}^{\text {cris }} \rightarrow \mathcal{O}_{n, \pi}^{\text {cris }}$ is precisely the operator denoted ${ }_{g} \phi_{1}$ earlier in this section (see (5.4.1)). Using this, together with Lemma 5.3.1. Lemma 5.4.4 (for $n=1$ ) and obvious functorialities, we obtain, after tensoring by $k[u] / u^{e \ell}$, the following corollary.

Corollary 5.4.5. Let $g: R \rightarrow R$ be a continuous automorphism.

(1) Let $\mathcal{G}$ be a finite flat group scheme over $R$, which is killed by $\ell$. Then there is a canonical isomorphism in the category $\underline{\phi_{1}-\bmod }{ }_{R}$ :

$$
\begin{aligned}
\left(\mathcal{M}_{\pi}(\mathcal{G}) \otimes_{k[u] / u^{e \ell}, \hat{g}} k[u] / u^{e \ell}, \mathcal{M}_{\pi}(\mathcal{G})_{1} \otimes_{k[u] / u^{e \ell, \hat{g}}} k[u] / u^{e \ell},{ }_{g} \phi_{1} \otimes \phi\right) & \\
& \stackrel{\sim}{\longrightarrow}\left(\mathcal{M}_{\pi}\left({ }^{g} \mathcal{G}\right), \mathcal{M}_{\pi}\left({ }^{g} \mathcal{G}\right)_{1}, \phi_{1}\right) .
\end{aligned}
$$

(2) If $f: \mathcal{G} \rightarrow \mathcal{G}^{\prime}$ is a morphism of finite flat $R$-group schemes killed by $\ell$ and $\mathcal{M}_{\pi}(f)$ is the corresponding morphism in $\phi_{1}-\bmod { }_{R}$, then $\mathcal{M}_{\pi}(f)$ also commutes with the ${ }_{g} \phi_{1}$ and there is a commutative diagram in $\underline{\phi_{1}-\bmod }{ }_{R}$ :

$$
\begin{array}{ccc}
\mathcal{M}_{\pi}\left(\mathcal{G}^{\prime}\right) \otimes_{k[u] / u^{e \ell, \hat{g}}}\left(k[u] / u^{e \ell}\right) & \stackrel{\mathcal{M}_{\pi(f)} \otimes 1}{\longrightarrow} & \mathcal{M}_{\pi}(\mathcal{G}) \otimes_{k[u] / u^{e \ell,}, \hat{g}}\left(k[u] / u^{e \ell}\right) \\
\mathcal{M}_{\pi}\left({ }^{g} \mathcal{G}^{\prime}\right) & \stackrel{\mathcal{M}_{\pi}\left({ }^{g} f\right)}{\longrightarrow} & \mathcal{M}_{\pi}\left({ }^{g} \mathcal{G}\right)
\end{array}
$$

(3) If $g_{1}, g_{2}$ are two continuous automorphisms of $R$ and if we choose the unique $H_{g_{2} g_{1}} \in W(k)[[u]]$ such that $\widehat{g_{2} g_{1}}=\widehat{g}_{2} \circ \widehat{g}_{1}$ on $W(k)[[u]]$, then on

$$
\begin{aligned}
& \left(\mathcal{M}_{\pi}(\mathcal{G}) \otimes_{k[u] / u^{e \ell}, \hat{g}_{1}} k[u] / u^{e \ell}\right) \otimes_{k[u] / u^{e \ell}, \hat{g}_{2}} k[u] / u^{e \ell} \simeq \mathcal{M}_{\pi}(\mathcal{G}) \otimes_{k[u] / u^{e \ell, \widehat{g_{2} g_{1}}}} k[u] / u^{e \ell}, \\
& \text { one has }{ }_{g_{2}}\left(g_{1} \phi_{1} \otimes \phi\right) \otimes \phi={ }_{g_{2} g_{1}} \phi_{1} \otimes \phi .
\end{aligned}
$$

Corollary 5.4.6. Let $\mathcal{G}$ be a finite flat group scheme over $R$, which is killed by $\ell$. To give a morphism of schemes $[g]: \mathcal{G} \rightarrow \mathcal{G}$ such that the diagram of schemes

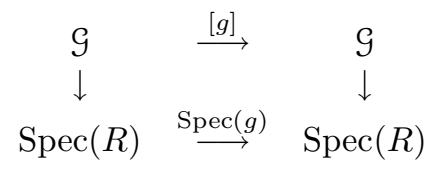


is commutative and the induced morphism $\mathcal{G} \rightarrow \operatorname{Spec}(R) \times_{g, \operatorname{Spec}(R)} \mathcal{G}$ is a morphism of group schemes over $R$, is equivalent to giving an additive map $\widehat{g}: \mathcal{M}_{\pi}(\mathcal{G}) \rightarrow$ $\mathcal{M}_{\pi}(\mathcal{G})$ such that both of the following hold:

(1) For all $s \in k[u] / u^{e \ell}$ and $x \in \mathcal{M}_{\pi}(\mathcal{G}), \widehat{g}(s x)=\widehat{g}(s) \widehat{g}(x)$.

(2) $\widehat{g}\left(\mathcal{M}_{\pi}(\mathcal{G})_{1}\right) \subset \mathcal{M}_{\pi}(\mathcal{G})_{1}$ and $\phi_{1} \circ \widehat{g}=\widehat{g} \circ \phi_{1}+\widehat{g}\left({ }_{g} t(u)\right) \widehat{g} \circ N \circ \phi_{1}$ with ${ }_{g} t$ as in Lemma 5.4.3 and $N$ as in Lemma 5.1.2.

Proof. Note that the last condition is equivalent to $\phi_{1} \circ \widehat{g}=\widehat{g} \circ{ }_{g} \phi_{1}$. The first two conditions are equivalent to giving a morphism $\widehat{g}: \mathcal{M}_{\pi}\left({ }^{g} \mathcal{G}\right) \rightarrow \mathcal{M}_{\pi}(\mathcal{G})$ in $\underline{\phi_{1}-\bmod }{ }_{R}$, which is equivalent to the last two by Corollary 5.4 .5 .

Finally we make some computations that concern the dependence of the above compatibilities on the choice of $H_{g}(u)$. Let $f(u)$ be an element of $\left(k[u] / u^{e \ell}\right)_{1}=$ $u^{e}\left(k[u] / u^{e \ell}\right)$ and define, for any $\mathcal{M}$ in ${\underline{\phi_{1}-\bmod }}_{R}$, the additive map $\mathbf{1}_{f}: \phi_{1}\left(\mathcal{M}_{1}\right) \rightarrow \mathcal{M}$ via

$$
\mathbf{1}_{f}=1+\left(\sum_{i=1}^{\ell-1} \frac{(-1)^{i-1}}{i} f(u)^{i}\right) N,
$$

where $N$ is as in Lemma 5.1.2. Using $k[u] / u^{e \ell} \otimes_{k\left[u^{\ell}\right] / u^{e \ell}} \phi_{1}\left(\mathcal{M}_{1}\right) \simeq \mathcal{M}$, we extend $\mathbf{1}_{f}$ to all of $\mathcal{M}$ by the formula:

$$
\mathbf{1}_{f}\left(u^{i} x\right)=u^{i}(1+f(u))^{i} \mathbf{1}_{f}(x)
$$

for $x \in \phi_{1}\left(\mathcal{M}_{1}\right)$. If $x \in \mathcal{M}_{1}$, one checks that:

$$
\mathbf{1}_{f}\left(\phi_{1}\left(u^{i} x\right)\right)=u^{i \ell} \mathbf{1}_{f}\left(\phi_{1}(x)\right)=\mathbf{1}_{f}\left(u^{i \ell} \phi_{1}(x)\right)
$$

so $\mathbf{1}_{f}$ is well defined. Moreover, it is clear that $\mathbf{1}_{f}\left(\mathcal{M}_{1}\right) \subset \mathcal{M}_{1}$. Let

$$
\mathbf{1}_{f}: \mathcal{O}_{1, \pi}^{\text {cris }} \stackrel{\sim}{\longrightarrow} \mathcal{O}_{1, \pi}^{\text {cris }}
$$

be the unique isomorphism of sheaves coming from the semi-linear isomorphism of presheaves:

$$
\begin{array}{ccc}
\left(k[u] / u^{e \ell}\right) \otimes_{\phi, k} \Gamma\left(\mathfrak{X}_{1}, \mathcal{O}_{\mathfrak{X}_{1}}\right) & \stackrel{\sim}{\longrightarrow}\left(k[u] / u^{e \ell}\right) \otimes_{\phi, k}\left(\Gamma\left(\mathfrak{X}_{1}, \mathcal{O}_{\mathfrak{X}_{1}}\right)\right) \\
s(u) \otimes\left(w_{0}, \ldots, w_{n-1}\right) & \longmapsto s(u(1+f(u))) \otimes\left(w_{0}, \ldots, w_{n-1}\right)
\end{array}
$$

(see 5.3.1).

Let $\mathcal{G}$ be a finite flat group scheme over $R$ killed by $\ell$ and recall that

$$
\mathcal{M}_{\pi}(\mathcal{G})=\operatorname{Hom}_{\text {sheaves of groups }}\left(\mathcal{G}, \mathcal{O}_{1, \pi}^{\text {cris }}\right) \otimes k[u] / u^{e \ell} .
$$

Lemma 5.4.7. The operator $\mathbf{1}_{f}$ on $\mathcal{M}_{\pi}(\mathcal{G})$ is induced by the operator $\mathbf{1}_{f}$ on $\mathcal{O}_{1, \pi}^{\text {cris }}$.

Proof. One can check that the operator $\mathbf{1}_{f}$ on $\mathcal{O}_{1, \pi}^{\text {cris }}$ satisfies $\mathbf{1}_{f} \circ \phi_{1}=\phi_{1}+$ $\log (1+f) N \circ \phi_{1}$, where $N$ is defined as in 5.3 and $\log (1+f)$ is the usual expansion of $\log$ in $S_{1}$, which makes sense because of the assumption that $u^{e} \mid f$ and because of the divided powers $\gamma_{i}\left(u^{e}\right)=\frac{u^{e i}}{i !}$. After tensoring with $k[u] / u^{e \ell}$, we get $\mathbf{1}_{f}=\mathbf{1}+\left(\sum_{i=1}^{\ell-1} \frac{(-1)^{i-1}}{i} f(u)^{i}\right) N$ on $\phi_{1}\left(\mathcal{M}_{\pi}(\mathcal{G})_{1}\right)$ which clearly implies the two $\mathbf{1}_{f}$ 's are the same.

Let $g=1$ and choose $H_{g}(u)=1+f(u)$ for some $f \in E_{\pi}(u) W(k)[[u]]$ (see the start of $\$ 5.1$ for the definition of $E_{\pi}(u)$ ). Recall from Corollary 5.4.5 that we have a canonical isomorphism $\mathcal{M}_{\pi}(\mathcal{G}) \otimes_{k[u] / u^{e \ell, \widehat{g}}}\left(k[u] / u^{\ell \ell}\right) \stackrel{\sim}{\rightarrow} \mathcal{M}_{\pi}\left({ }^{g} \mathcal{G}\right)$. 
Lemma 5.4.8. The map $\mathbf{1}_{f}$ is the composite $\mathcal{M}_{\pi}(\mathcal{G}) \stackrel{\sim}{\rightarrow} \mathcal{M}_{\pi}\left({ }^{g} \mathcal{G}\right) \stackrel{\sim}{\rightarrow} \mathcal{M}_{\pi}(\mathcal{G})$, where the first map is the one in Corollary 5.4 .5 and the second comes from the obvious isomorphism $\mathcal{G} \stackrel{\sim}{\rightarrow} g \mathcal{G}$. In other words, once $H_{g}(u)=1+f(u)$ has been chosen, $\mathbf{1}_{f}: \mathcal{M}_{\pi}(\mathcal{G}) \rightarrow \mathcal{M}_{\pi}(\mathcal{G})$ is the map corresponding to the identity $\mathbf{1}_{\mathcal{G}}: \mathcal{G} \rightarrow \mathcal{G}$ under the equivalence of Corollary 5.4.6.

The proof is straightforward by looking at the usual presheaves and using Lemma 5.4.7. We remark that $\mathbf{1}_{f}$ is not necessarily the identity even though $1_{g}$ is. However, with $f=0, \mathbf{1}_{f}$ is the identity.

5.5. Reformulation. In this section, we will reformulate Corollary 5.4.6,

Lemma 5.5.1. There is a unique element $t_{g}(u) \in W(k)[[u]]$ such that if $\phi_{g}$ is defined by $\phi_{g}\left(\sum w_{i} u^{i}\right)=\sum \phi\left(w_{i}\right)\left(u^{\ell}\left(1+\ell t_{g}(u)\right)\right)^{i}$, one has $\widehat{g} \circ \phi=\phi_{g} \circ \widehat{g}$.

Proof. One has to solve in $W(k)[[u]]$ :

$$
u^{\ell} H_{g}(u)^{\ell}=u^{\ell}\left(1+\ell t_{g}(u)\right)^{(\phi)} H_{g}\left(u^{\ell}\left(1+\ell t_{g}(u)\right)\right) .
$$

As $H_{g}(u) \in W(k)[[u]]^{\times}$, there is a unique $L_{g} \in u W(k)[[u]]^{\times}$such that $L_{g}\left(u H_{g}(u)\right)=$ $u$. Applying $L_{g}$ to $u=K_{g}(u) H_{g}\left(K_{g}(u)\right.$ ) (cf. the proof of Lemma 5.4.3), we get $L_{g}(u)=K_{g}(u)$. We must solve:

$$
1+\ell t_{g}(u)=\frac{{ }^{(\phi)} K_{g}\left(u^{\ell} H_{g}(u)^{\ell}\right)}{u^{\ell}} .
$$

Lemma 5.5.2. There is a unique $\lambda_{g}(u) \in 1+u W(k)[[u]]$ such that if $N_{g}=\lambda_{g} N$, then $N_{g} \circ \widehat{g}=\widehat{g} \circ N$. Similarly, there is a unique ${ }_{g} \lambda(u) \in 1+u W(k)[[u]]$ such that if ${ }_{g} N={ }_{g} \lambda N$, then $\widehat{g} \circ{ }_{g} N=N \circ \widehat{g}$. Moreover, $N_{g} \circ \phi_{g}=\ell \phi_{g} \circ N_{g}$ and ${ }_{g} N \circ{ }_{g} \phi=\ell_{g} \phi \circ{ }_{g} N$.

Proof. Since $N$ is a derivation, so is $\lambda N$ for any $\lambda \in W(k)[[u]]$. One has to solve $\lambda_{g}(u) N\left(u H_{g}(u)\right)=u H_{g}(u)$ and ${ }_{g}^{(g)} \lambda\left(u H_{g}(u)\right)=1+\frac{N\left(H_{g}(u)\right)}{H_{g}(u)}$, which amounts to:

$$
\begin{aligned}
\lambda_{g}(u) & =\left(1+\frac{N\left(H_{g}(u)\right)}{H_{g}(u)}\right)^{-1}, \\
{ }_{g}^{(g)} \lambda(u) & =1+\frac{N\left(H_{g}\right)\left(K_{g}(u)\right)}{H_{g}\left(K_{g}(u)\right)},
\end{aligned}
$$

where $K_{g}$ is as in the proof of Lemma 5.4.3. The commutation relations with the Frobenius follow from $N \circ \phi=\ell \phi \circ N, \phi_{g} \circ \widehat{g}=\widehat{g} \circ \phi, N_{g} \circ \widehat{g}=\widehat{g} \circ N, \widehat{g} \circ{ }_{g} \phi=\phi \circ \widehat{g}$, $\widehat{g} \circ{ }_{g} N=N \circ \widehat{g}$ and the fact $\widehat{g}$ is bijective on $W(k)[[u]]$.

We also denote by ${ }_{g} N={ }_{g} \lambda N$ and $N_{g}=\lambda_{g} N$ the corresponding derivations on $k[u] / u^{e \ell}$. For any object $\mathcal{M}$ of ${\underline{\phi_{1}-\bmod }}_{R}$, define $\phi_{1, g}: \mathcal{M}_{1} \rightarrow \mathcal{M}$ by the formula

$$
\phi_{1, g}(x)=\phi_{1}(x)+t_{g}(u) N\left(\phi_{1}(x)\right) \text {, }
$$

where $N$ is as in Lemma 5.1.2, and we recall that we defined ${ }_{g} \phi_{1}$ in (5.4.1). One checks that $\phi_{1, g}\left(u^{e}\right)={ }_{g} \phi_{1}\left(u^{e}\right)=\phi_{1}\left(u^{e}\right)=c_{\pi}$ (see (5.1.1)). Note that we also have $\phi_{1, g} \circ \widehat{g}=\widehat{g} \circ{ }_{g} \phi_{1}, N_{g} \circ \widehat{g}=\widehat{g} \circ N, \widehat{g} \circ{ }_{g} \phi_{1}=\phi_{1} \circ \widehat{g}, \widehat{g} \circ{ }_{g} N=N \circ \widehat{g}$ in $k[u] / u^{e \ell}$.

Lemma 5.5.3. Let $\mathcal{M}$ be an object of ${\underline{\phi_{1}-\bmod _{R}}}_{R}$; then there is a unique operator $N_{g}: \mathcal{M} \rightarrow \mathcal{M}$ satisfying the following three conditions:

(1) $N_{g}(s x)=N_{g}(s) x+s N_{g}(x), s \in k[u] / u^{e \ell}, x \in \mathcal{M}$, 
(2) $N_{g} \phi_{1, g}(x)=\phi_{g} N_{g}(x), x \in \mathcal{M}_{1}$, where $\phi_{g}(y)=\frac{1}{c_{\pi}} \phi_{1, g}\left(u^{e} y\right)$ if $y \in \mathcal{M}$,

(3) $N_{g}(\mathcal{M}) \subset u \mathcal{M}$.

The same statement holds for ${ }_{g} N,{ }_{g} \phi$ and ${ }_{g} \phi_{1}$.

Proof. The proof is the same as for Lemma 5.1.2 using the fact we still have isomorphisms

$$
k[u] / u^{e \ell} \otimes_{k\left[u^{\ell}\right] / u^{e \ell}} \phi_{1, g}\left(\mathcal{M}_{1}\right) \stackrel{\sim}{\rightarrow} \mathcal{M}
$$

(resp. with ${ }_{g} \phi_{1}$ replacing $\phi_{1, g}$ ).

Lemma 5.5.4. For $\mathcal{M}$ an object of $\phi_{1}-\bmod { }_{R}, N_{g}=\lambda_{g} N$ and ${ }_{g} N={ }_{g} \lambda N$, where $N_{g},{ }_{g} N$ are as in Lemma 5.5.3, $\lambda_{g},{ }_{g} \lambda$ as in Lemma 5.5.2 and $N$ as in Lemma 5.1 .2 .

Proof. By unicity of $N_{g}$, one has to check $\lambda_{g} N$ satisfies the three conditions of Lemma 5.5.3 The first and last are obvious. Note that $N \phi_{1}\left(u^{e} x\right)=\phi N\left(u^{e} x\right)=0$ so $\phi_{1, g}\left(u^{e} x\right)=\phi_{1}\left(u^{e} x\right)$, which implies $\phi=\phi_{g}$ on $\mathcal{M}\left(\phi_{g}\right.$ is as in Lemma 5.5.3). One computes:

$$
\begin{aligned}
\left(\lambda_{g}(u) N\right) \circ \phi_{1, g} & =\lambda_{g}(u)\left(1+N\left(t_{g}(u)\right)\right) \phi \circ N, \\
\phi_{g} \circ\left(\lambda_{g}(u) N\right) & ={ }^{(\phi)} \lambda_{g}\left(u^{\ell}\right) \phi \circ N .
\end{aligned}
$$

But the equality $N_{g} \circ \phi_{g}(u)=\ell \phi_{g} \circ N_{g}(u)$ in $W(k)[[u]]$ (from Lemma 5.5.2) yields

$$
\lambda_{g}(u)\left(1+N\left(t_{g}(u)\right)\right)-{ }^{(\phi)} \lambda_{g}\left(u^{\ell}\right) \in \ell W(k)[[u]] .
$$

We thus get $\left(\lambda_{g} N\right) \circ \phi_{1, g}=\phi_{g} \circ\left(\lambda_{g} N\right)$, hence condition (2). For ${ }_{g} N$, the proof is completely similar.

Lemma 5.5.5. Let $\mathcal{M}$ be an object of ${\underline{\phi_{1}-\bmod _{R}}}_{R}$ and $\widehat{g}: \mathcal{M} \rightarrow \mathcal{M}$ an additive map such that for all $s \in k[u] / u^{e \ell}$ and $x \in \mathcal{M}, \widehat{g}(s x)=\widehat{g}(s) \widehat{g}(x)$ and $\widehat{g}\left(\mathcal{M}_{1}\right) \subset \mathcal{M}_{1}$. If $\widehat{g} \circ \phi_{1}=\phi_{1, g} \circ \widehat{g}$, then $\widehat{g} \circ N=N_{g} \circ \widehat{g}$. Similarly, if $\phi_{1} \circ \widehat{g}=\widehat{g} \circ{ }_{g} \phi_{1}$, then $N \circ \widehat{g}=\widehat{g} \circ{ }_{g} N$.

Proof. We prove the first case, the other one being the same. As in the proof of Lemma 5.1.2 we define $N_{g, 0}, N_{g, 1}, \ldots$, with $N_{g}=N_{g, i}$ for $i$ large enough, using $k[u] / u^{e \ell} \otimes_{k\left[u^{\ell}\right] / u^{e \ell}} \phi_{1, g}\left(\mathcal{M}_{1}\right) \stackrel{\sim}{\rightarrow} \mathcal{M}$. It is enough to show $\widehat{g} \circ N_{i}=N_{g, i} \circ \widehat{g}$ for all $i$. Suppose $\widehat{g} \circ N_{i-1}=N_{g, i-1} \circ \widehat{g}$ and let $s \in k[u] / u^{e \ell}$ and $x \in \mathcal{M}_{1}$. Then

$$
\begin{aligned}
N_{g, i} \widehat{g}\left(s \phi_{1}(x)\right) & =N_{g, i}\left(\widehat{g}(s) \phi_{1, g}(\widehat{g}(x))\right) \\
& =N_{g}(\widehat{g}(s)) \phi_{1, g}(\widehat{g}(x))+\widehat{g}(s) \phi_{1, g} N_{g, i-1}(\widehat{g}(x)) \\
& =\widehat{g}\left(N(s) \phi_{1}(x)\right)+\widehat{g}(s) \phi_{1, g} \widehat{g}\left(N_{i-1}(x)\right) \\
& =\widehat{g}\left(N(s) \phi_{1}(x)\right)+\widehat{g}\left(s \phi_{1}\left(N_{i-1}(x)\right)\right) \\
& =\widehat{g} N_{i}\left(s \phi_{1}(x)\right),
\end{aligned}
$$

so $\widehat{g} \circ N_{i}=N_{g, i} \circ \widehat{g}$ by linearity. One easily checks by a similar computation that $N_{g, 0} \circ \widehat{g}=\widehat{g} \circ N_{0}$, hence the result follows by induction.

Lemma 5.5.6. Let $\mathcal{M}$ be an object of ${\underline{\phi_{1}-\bmod _{R}}}_{R}$ and $\widehat{g}: \mathcal{M} \rightarrow \mathcal{M}$ an additive map such that for all $s \in k[u] / u^{e \ell}$ and $x \in \mathcal{M}, \widehat{g}(s x)=\widehat{g}(s) \widehat{g}(x)$ and $\widehat{g}\left(\mathcal{M}_{1}\right) \subset \mathcal{M}_{1}$. Then the following two conditions are equivalent:

(1) $\phi_{1} \circ \widehat{g}=\widehat{g} \circ \phi_{1}+\widehat{g}\left({ }_{g} t(u)\right) \widehat{g} \circ N \circ \phi_{1}$,

(2) and $\widehat{g} \circ \phi_{1}=\phi_{1} \circ \widehat{g}+t_{g}(u) N \circ \phi_{1} \circ \widehat{g}$. 
Proof. One has to show $\phi_{1} \circ \widehat{g}=\widehat{g} \circ{ }_{g} \phi_{1}$ is equivalent to $\widehat{g} \circ \phi_{1}=\phi_{1, g} \circ \widehat{g}$. We prove $(1) \Rightarrow(2)$, the other case being the same. On $\mathcal{M}$, we have $\widehat{g} \circ \phi=\phi \circ \widehat{g}$, because $\phi=\phi_{g}={ }_{g} \phi$, as in the proof of Lemma 5.5.4. By Lemmas 5.5.4 and 5.5.5, we have $\widehat{g} \circ N=\widehat{g}\left({ }_{g} \lambda^{-1}\right) N \circ \widehat{g}$. Thus we get from (1), using $N \phi_{1}=\phi N$,

$$
\widehat{g} \circ \phi_{1}=\phi_{1} \circ \widehat{g}-\widehat{g}\left({ }_{g} t(u)\right) \widehat{g}\left({ }_{g} \lambda(u)^{-1}\right)^{\ell} N \circ \phi_{1} \circ \widehat{g} .
$$

Playing the same game over $W_{2}(k)[[u]]$ with the relation $\phi \circ \widehat{g}=\widehat{g} \circ \phi+\widehat{g}\left({ }_{g} t(u)\right) \widehat{g} \circ$ $N \circ \phi$, which is easily checked to hold in $W_{2}(k)[[u]]$, we again end up with $\widehat{g} \circ \phi=$ $\phi \circ \widehat{g}-\widehat{g}\left({ }_{g} t(u)\right) \widehat{g}\left({ }_{g} \lambda(u)^{-1}\right)^{\ell} N \circ \phi \circ \widehat{g}$ in $W_{2}(k)[[u]]$. But we also have in $W_{2}(k)[[u]]$ the equality:

$$
\widehat{g} \circ \phi=\phi \circ \widehat{g}+t_{g}(u) N \circ \phi \circ \widehat{g} .
$$

Thus $-\widehat{g}\left({ }_{g} t(u)\right) \widehat{g}\left({ }_{g} \lambda(u)^{-1}\right)^{\ell}=t_{g}(u)$ in $k[u] / u^{e \ell}$, so relation (2) holds.

We can now derive the variant of Corollary 5.4.6 which we will use.

Corollary 5.5.7. Let $\mathcal{G}$ be a finite flat $R$-group scheme killed by $\ell$. Let $g: R \stackrel{\sim}{\rightarrow} R$ be a continuous automorphism, choose $H_{g}(u) \in W(k)[[u]]$ such that $g(\pi)=\pi H_{g}(\pi)$ and define $\widehat{g}: k[u] / u^{e \ell} \rightarrow k[u] / u^{e \ell}$ by $\widehat{g}\left(\sum w_{i} u^{i}\right)=\sum g\left(w_{i}\right) u^{i} H_{g}(u)^{i}$. To give a morphism of schemes $[g]: \mathcal{G} \rightarrow \mathcal{G}$ such that the diagram of schemes

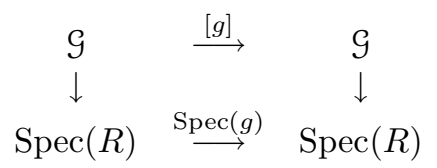

is commutative and the induced morphism $\mathcal{G} \rightarrow \operatorname{Spec}(R) \times_{g, \operatorname{Spec}(R)} \mathcal{G}$ is an morphism of group schemes over $R$, is equivalent to giving an additive map $\widehat{g}: \mathcal{M}_{\pi}(\mathcal{G}) \rightarrow$ $\mathcal{M}_{\pi}(\mathcal{G})$ such that both of the following hold:

(1) For all $s \in k[u] / u^{e \ell}$ and $x \in \mathcal{M}_{\pi}(\mathcal{G}), \widehat{g}(s x)=\widehat{g}(s) \widehat{g}(x)$.

(2) $\widehat{g}\left(\mathcal{M}_{\pi}(\mathcal{G})_{1}\right) \subset \mathcal{M}_{\pi}(\mathcal{G})_{1}$ and $\widehat{g} \circ \phi_{1}=\left(1+t_{g}(u) N\right) \circ \phi_{1} \circ \widehat{g}$, with $t_{g}$ as in Lemma 5.5 .1 and $N$ as in Lemma 5.1.2.

Moreover, $[g]$ is an isomorphism if and only if $\widehat{g}$ is. Assume these are isomorphisms. Choose $H_{g^{-1}}$ such that $\widehat{g^{-1}}(u)=\widehat{g}^{-1}(u)$ on $W(k)[[u]]$, i.e. $H_{g^{-1}}(u)=\widehat{g}^{-1}(u) / u$. Then the map $\widehat{g^{-1}}$ that corresponds to $[g]^{-1}$ is equal to $\widehat{g}^{-1}$. Also, if $g_{1}, g_{2}$ are two automorphisms of $R$ and if we choose $H_{g_{1}}, H_{g_{2}}$ as above, then $\left[g_{1}\right] \circ\left[g_{2}\right]$ corresponds to $\widehat{g}_{2} \circ \widehat{g}_{1}$ provided we choose $H_{g_{2} g_{1}}$ such that $\widehat{g}_{2}\left(\widehat{g}_{1}(u)\right)=u H_{g_{2} g_{1}}(u)$.

Proof. The equivalence is clear thanks to Corollary 5.4.6 and Lemma 5.5.6. The fact that $\left[g_{1}\right] \circ\left[g_{2}\right]$ corresponds to $\widehat{g}_{2} \circ \widehat{g}_{1}$ is automatic using Corollary 5.4.5 and the functor $\mathcal{G} \mapsto \mathcal{M}_{\pi}(\mathcal{G})$. Applying this to $g_{1}=g$ and $g_{2}=g^{-1}$, we see that $1_{\mathcal{G}}=[g] \circ[g]^{-1}$ corresponds to $\widehat{g^{-1}} \circ \widehat{g}$. But by Lemma 5.4.8, $1_{g}$ corresponds to $\mathbf{1}_{f}$ with $f$ defined by $\left(\widehat{g^{-1}} \circ \widehat{g}\right)(u)=u(1+f)$ in $W(k)[[u]]$. We see that $f=0$ and that $\mathbf{1}_{f}$ is the identity on $\mathcal{M}_{\pi}(\mathcal{G})$. Thus $\widehat{g^{-1}}=\widehat{g}^{-1}$ on $\mathcal{M}_{\pi}(\mathcal{G})$.

5.6. Descent data. Assume now that $R$ is endowed with a continuous left faithful action of a finite group $\Gamma$. Then $\Gamma$ becomes the Galois group of the fraction field $F^{\prime}$ of $R$ over some subfield. For each $g \in \Gamma$, choose $H_{g}(u) \in W(k)[u]$ so that $g(\pi)=\pi H_{g}(\pi)$, with the one condition that $H_{1}(u)=1$. Recall from Lemma 5.5.1 that this uniquely determines elements $t_{g}(u) \in W(k)[[u]]$ such that

$$
u^{\ell} H_{g}(u)^{\ell}=u^{\ell}\left(1+\ell t_{g}(u)\right)^{(\phi)} H_{g}\left(u^{\ell}\left(1+\ell t_{g}(u)\right)\right) .
$$


Moreover, for any pair $g_{1}, g_{2} \in \Gamma$, there is obviously a unique $f_{g_{1}, g_{2}}(u) \in$ $E_{\pi}(u) W(k)[[u]]$ such that

$$
\widehat{g}_{1} \circ \widehat{g}_{2}(u)=\widehat{g_{1} \circ g_{2}}\left(u\left(1+f_{g_{1}, g_{2}}(u)\right)\right) .
$$

If $\mathcal{M}$ is an object of ${\underline{\phi_{1}}-\bmod }_{R}$, then we will denote by $\mathbf{1}_{g_{1}, g_{2}}$ the unique $k$-linear map $\mathcal{M} \rightarrow \mathcal{M}$ such that for $x \in \mathcal{M}_{1}$ we have

- $\mathbf{1}_{g_{1}, g_{2}}\left(\phi_{1}(x)\right)=\left(1+\left(\sum_{i=1}^{\ell-1} \frac{(-1)^{i-1}}{i} f_{g_{1}, g_{2}}(u)^{i}\right) N\right)\left(\phi_{1}(x)\right)$, where $N$ is as in Lemma 5.1.2.

- and $\mathbf{1}_{g_{1}, g_{2}}\left(u^{i} \phi_{1}(x)\right)=u^{i}\left(1+f_{g_{1}, g_{2}}(u)\right)^{i} \mathbf{1}_{g_{1}, g_{2}}\left(\phi_{1}(x)\right)$.

(See $\$ 5.4$ where we denoted $\mathbf{1}_{g_{1}, g_{2}}$ by $\mathbf{1}_{f_{g_{1}, g_{2}}}$.)

Suppose that $\mathcal{G}$ is a finite flat $R$-group scheme. Recall that by descent data on $\mathcal{G}$ for $\Gamma$ we mean isomorphisms of finite flat group schemes

$$
[g]: \mathcal{G} \stackrel{\sim}{\rightarrow} g \mathcal{G}
$$

for $g \in \Gamma$, such that

$$
[g h]=\left({ }^{g}[h]\right) \circ[g]
$$

for all $g, h \in \Gamma$. Equivalently we may think of $[g]$ as a map of schemes $\mathcal{G} \rightarrow \mathcal{G}$ over $g^{*}: \operatorname{Spec} R \rightarrow \operatorname{Spec} R$ which induces an isomorphism of group schemes $\mathcal{G} \rightarrow{ }^{g} \mathcal{G}$. In this picture the compatibility condition simply becomes

$$
[g h]=[h][g] .
$$

Theorem 5.6.1. Suppose that $\mathcal{G}$ is a finite flat $R$-group scheme killed by $\ell$. Fix $H_{g}(u)$ as above for all $g \in \Gamma$.

(1) To give descent data on $\mathcal{G}$ relative to $\Gamma$ is equivalent to giving additive bijections $\widehat{g}: \mathcal{M}_{\pi}(\mathcal{G}) \rightarrow \mathcal{M}_{\pi}(\mathcal{G})$ for all $g \in \Gamma$ so that $\widehat{g}$ takes $\mathcal{M}_{\pi}(\mathcal{G})_{1}$ into $\mathcal{M}_{\pi}(\mathcal{G})_{1}$ and:

- $\widehat{g}\left(w u^{i} m\right)=g(w)\left(u H_{g}(u)\right)^{i} \widehat{g}(m)$ for $m \in \mathcal{M}_{\pi}(\mathcal{G}), w \in k$,

- $\widehat{g} \circ \phi_{1}=\left(1+t_{g}(u) N\right) \circ \phi_{1} \circ \widehat{g}$ on $\mathcal{M}_{\pi}(\mathcal{G})_{1}$,

- $\widehat{1}_{\Gamma}=1$ and $\widehat{g}_{1} \circ \widehat{g}_{2}={\widehat{g_{1} g_{2}}}_{\mathbf{1}_{g_{1}, g_{2}}}$.

(2) The above equivalence is functorial in $\mathcal{G}$ and is compatible with classical Dieudonné theory in the following sense: if the action $\{\widehat{g}\}_{g \in \Gamma}$ on $\mathcal{M}_{\pi}(\mathcal{G})$ corresponds to descent data $\{[g]\}$ on $\mathcal{G}$, then the g-semi-linear map $D([g])$ induced on the contravariant Dieudonné module $D(\mathcal{G})$ and the g-semi-linear map $\widehat{g} \bmod u$ induced on $\mathcal{M}_{\pi}(\mathcal{G}) / u \mathcal{M}_{\pi}(\mathcal{G})$ are compatible via the isomorphism of Theorem 5.1.3.

Proof. Part (1) is a consequence of Corollary 5.5.7 Lemma 5.4.8 and the choice $H_{1}=1$. The functoriality in (2) follows from Corollary 5.4.5, and the last statement there comes from ${ }^{g}(\mathcal{G} \times k) \cong g \mathcal{G} \times k$, the functoriality of the isomorphism in Theorem 5.1 .3 and the reduction modulo $u$ of Corollary 5.4.5.

Suppose that $\pi \in R^{\Gamma}$. Then we may take $H_{g}(u)=1$ for all $g \in \Gamma$. With this choice we see that $t_{g_{1}}=0, f_{g_{1}, g_{2}}=0$ and $\mathbf{1}_{g_{1}, g_{2}}=1$ for all $g_{1}, g_{2} \in \Gamma$. In this case to give bijections $\widehat{g}: \mathcal{M}_{\pi}(\mathcal{G}) \rightarrow \mathcal{M}_{\pi}(\mathcal{G})$ as in the lemma is equivalent to giving an $R$-semi-linear $\Gamma$-action on $\mathcal{M}_{\pi}(\mathcal{G})$ which commutes with $u$ and $\phi_{1}$ and preserves $\mathcal{M}_{\pi}(\mathcal{G})_{1}$. Thus $\left(\mathcal{M}_{\pi}(\mathcal{G})^{\Gamma}, \mathcal{M}_{\pi}(\mathcal{G})_{1}^{\Gamma}, \phi_{1}\right)$ is a Breuil module over $R^{\Gamma}$ from which we can recover $\mathcal{M}_{\pi}(\mathcal{G})$ by tensoring with $W(k)$ over $W\left(k^{\Gamma}\right)$. In other words, étale descent for group schemes translates in the obvious manner for Breuil modules if we choose $\pi$ to be $\Gamma$-invariant. 
To build an action of $\Gamma$ on $\mathcal{G}$ using Theorem 5.6.1, the conditions $\widehat{g}_{1} \circ \widehat{g}_{2}=\widehat{g_{1} g_{2}} \circ$ $\mathbf{1}_{g_{1}, g_{2}}$ are not very convenient to check in practice since there are too many of them. It is useful to have the following variant. Choose $d \in \mathbf{Z}_{>0}$ and a group surjection $\theta: \Gamma_{d} \rightarrow \Gamma$, where $\Gamma_{d}$ is the free group on $d$ generators $\gamma_{1}, \ldots, \gamma_{d}$. The group $\Gamma_{d}$ still acts on $R$ (via its quotient $\Gamma$ ) and for each $i \in\{1, \ldots, d\}$, choose elements $H_{\gamma_{i}}(u) \in W(k)[[u]]$ such that $\pi H_{\gamma_{i}}(\pi)=\gamma_{i}(\pi)$. This determines isomorphisms $\widehat{\gamma}_{i}$ on $W(k)[[u]]$ and $k[u] / u^{e \ell}$ and, by composition, isomorphisms $\widehat{\gamma}$ for all $\gamma \in \Gamma_{d}$. Note that if $\gamma \in \operatorname{ker}(\theta)$, then $H_{\gamma}(u)=u\left(1+f_{\gamma}(u)\right)$ for some $f_{\gamma} \in E_{\pi}(u) W(k)[[u]]$. For such $\gamma$, denote by $\mathbf{1}_{\gamma}$ the unique $k$-vector space endomorphism of any object $\mathcal{M}$ of $\phi_{1}-\bmod { }_{R}$ such that for $x \in \mathcal{M}_{1}$ we have

- $\mathbf{1}_{\gamma}=1+\left(\sum_{i=1}^{\ell-1} \frac{(-1)^{i-1}}{i} f_{\gamma}(u)^{i}\right) N$ on the image of $\phi_{1}$,

- and $\mathbf{1}_{\gamma}\left(u^{i} \phi_{1}(x)\right)=u^{i}\left(1+f_{\gamma}(u)\right)^{i} \mathbf{1}_{\gamma}\left(\phi_{1}(x)\right)$,

where $N$ is as in Lemma 5.1.2. (See $\$ 5.4$, where we denoted $\mathbf{1}_{\gamma}$ by $\mathbf{1}_{f_{\gamma}}$.) Let $\mathcal{R}$ be a subset of $\operatorname{ker}(\theta)$ such that $\operatorname{ker}(\theta)$ is the smallest normal subgroup of $\Gamma_{d}$ containing $\mathcal{R}$.

Corollary 5.6.2. With the above notation, to give descent data on $\mathcal{G}$ for $\Gamma$ is equivalent to giving additive bijections $\widehat{\gamma_{j}}: \mathcal{M}_{\pi}(\mathcal{G}) \rightarrow \mathcal{M}_{\pi}(\mathcal{G})$ for $j \in\{1, \ldots, d\}$ so that $\widehat{\gamma_{j}}$ takes $\mathcal{M}_{\pi}(\mathcal{G})_{1}$ into $\mathcal{M}_{\pi}(\mathcal{G})_{1}$ and:

- $\widehat{\gamma}_{j}\left(w u^{i} m\right)=\gamma_{j}(w)\left(u H_{\gamma_{j}}(u)\right)^{i} \widehat{\gamma}_{j}(m)$ for $m \in \mathcal{M}_{\pi}(\mathcal{G}), w \in k$,

- $\widehat{\gamma}_{j} \circ \phi_{1}=\left(1+t_{\gamma_{j}}(u) N\right) \circ \phi_{1} \circ \widehat{\gamma}_{j}$ on $\mathcal{M}_{\pi}(\mathcal{G})_{1}$,

- if $\gamma=\gamma_{i_{1}}^{n_{1}} \ldots \gamma_{i_{m}}^{n_{m}} \in \mathcal{R}$, where $i_{j} \in\{1, \ldots, d\}, n_{j} \in \mathbf{Z}$, and $i_{j} \neq i_{j+1}$ for $1 \leq j<m$, and if we define $\widehat{\gamma}=\widehat{\gamma}_{i_{1}}^{n_{1}} \circ \cdots \circ \widehat{\gamma}_{i_{m}}^{n_{m}}$, then $\widehat{\gamma}=\mathbf{1}_{\gamma}$.

Proof. Straightforward from Corollary 5.5.7 and Lemma 5.4.8.

We define a category $\underline{\phi}_{1} D D_{F^{\prime} /\left(F^{\prime}\right)^{\Gamma}}$ of Breuil modules with descent data for $\Gamma$ in the obvious way. This category is additive but not necessarily abelian. We call a complex in $\underline{\phi}_{1} D D_{F^{\prime} /\left(F^{\prime}\right)^{\Gamma}}$ exact if the underlying complex in $\underline{\phi_{1}-\bmod } R$ is exact. In the natural way, we extend $\mathcal{M}_{\pi}$ to a functor from $\mathcal{F} \mathcal{D}_{F^{\prime} /\left(F^{\prime}\right)^{\Gamma}}$ to $\underline{\phi}_{1} D D_{F^{\prime} /\left(F^{\prime}\right)^{\Gamma}}$.

5.7. More examples. In this section we will determine the possible descent data on a rank one Breuil module. Let $\Gamma$ be as in $\$ 5.6$.

Lemma 5.7.1. Suppose that $\mathcal{G}$ is a finite flat $R$-group scheme of order $\ell$ and that its generic fibre admits descent data over $\left(F^{\prime}\right)^{\Gamma}$. Then there is unique descent data on $\mathcal{G}$ over $\left(F^{\prime}\right)^{\Gamma}$ extending any choice of descent data on $\mathcal{G} \times F^{\prime}$ over $\left(F^{\prime}\right)^{\Gamma}$. If $\mathcal{M}_{\pi}(\mathcal{G}) \cong \mathcal{M}(r, a)$ and if $\gamma \in \Gamma$ satisfies $\gamma(\pi) / \pi \equiv 1 \bmod (\pi)$, then

$$
\widehat{\gamma}(\mathbf{e})=H_{\gamma}(u)^{-r \ell /(\ell-1)} \mathbf{e}
$$

where $H_{\gamma}(u)^{-r \ell /(\ell-1)}$ denotes the unique $(\ell-1)^{\text {th }}$ root of $H_{\gamma}(u)^{-r \ell}$ in $k[u] / u^{e \ell}$ with constant term 1.

We remark that since $\operatorname{Aut}(\mathcal{M}(r, a))=(\mathbf{Z} / \ell \mathbf{Z})^{\times}$by consideration of the geometric generic fibre, the choice of isomorphism $\mathcal{M}_{\pi}(\mathcal{G}) \cong \mathcal{M}(r, a)$ does not matter.

Proof. We first claim two such finite flat group schemes $\mathcal{G}$ and $\mathcal{G}^{\prime}$ have isomorphic generic fibres if and only if there is a non-zero morphism $\mathcal{G} \rightarrow \mathcal{G}^{\prime}$ or $\mathcal{G}^{\prime} \rightarrow \mathcal{G}$. By Lemma 5.2.1 we see that if $G$ is a finite flat $F^{\prime}$-group scheme, then the lattice of models for $G$ over $R$ is well ordered. Suppose all the integral models are $\mathcal{G}_{1}<\cdots<$ 
$\mathcal{G}_{n}$. For $\gamma \in \Gamma$, any isomorphism $[\gamma]: G \stackrel{\sim}{\rightarrow} \gamma G$ must then induce isomorphisms $[\gamma]: \mathcal{G}_{i} \stackrel{\sim}{\rightarrow} \gamma \mathcal{G}_{i}$ for all $i=1, \ldots, n$. The first part of the lemma follows.

Let $\mathcal{M}=\mathcal{M}(r, a)$, so $\mathcal{M}$ is a free $k[u] / u^{e \ell}$-module of rank 1 with the usual basis element $\mathbf{e}$. The submodule $\mathcal{M}_{1}$ is spanned by $u^{r} \mathbf{e}$ and $\phi_{1}\left(u^{r} \mathbf{e}\right)=a \mathbf{e}$. From Theorem 5.2.1, we have $N \circ \phi_{1}=0$, which implies that

$$
\widehat{\gamma} \circ \phi_{1}=\phi_{1} \circ \widehat{\gamma}
$$

For $\gamma \in \Gamma_{1}, H_{\gamma}(0) \equiv 1 \bmod \ell$. Clearly

$$
\widehat{\gamma}: c u^{i} \mathbf{e} \longmapsto c u^{i} H_{\gamma}(u)^{i} \widehat{\gamma}(\mathbf{e})
$$

is a bijection if and only if $\widehat{\gamma}(\mathbf{e})=\xi_{\gamma} \mathbf{e}$ for some unit $\xi_{\gamma} \in\left(k[u] / u^{e \ell}\right)^{\times}$. Evaluating $\widehat{\gamma} \circ \phi_{1}^{\prime}=\phi_{1}^{\prime} \circ \widehat{\gamma}$ on the element $u^{r} \mathbf{e} \in \mathcal{M}_{1}$, we get

$$
\xi_{\gamma}=H_{\gamma}(u)^{r \ell} \xi_{\gamma}^{\ell}
$$

in $k[u] / u^{e \ell}$. Thus,

$$
\xi_{\gamma}=\epsilon_{\gamma} H_{\gamma}^{-r \ell /(\ell-1)}
$$

for some unit $\epsilon_{\gamma} \in \mathbf{F}_{\ell}^{\times}$.

Since Breuil module descent data always induces a $k$-linear action of the inertia group on the $k$-vector space $\mathcal{M} / u \mathcal{M}$ and in this case $\operatorname{dim}_{k} \mathcal{M} / u \mathcal{M}=1$, the action of the element $\gamma$ of $\ell$-power order on $\mathcal{M} / u \mathcal{M}$ must be trivial. Thus $\epsilon_{\gamma}=1$.

\section{Some LOCAL FIELDS}

In order to apply the methods of $\$ 15$, we need some more explicit information about the fields $F^{\prime}$ introduced in 4 . In this section we will collect this essentially elementary information. In each case we will give an explicit description of the Galois group $\operatorname{Gal}\left(F^{\prime} / \mathbf{Q}_{3}\right)$. This is needed to carry out the delicate Breuil module calculations in subsequent sections. We will also specify a uniformiser $\pi$ of $F^{\prime}$ and partially calculate the following polynomials and power series (depending on our choice of $\pi)$.

- $G(u) \in W\left(k_{F^{\prime}}\right)[u]$, a polynomial of degree at most $e\left(F^{\prime} / \mathbf{Q}_{3}\right)-1$ such that $\pi$ has minimal polynomial $u^{e\left(F^{\prime} / \mathbf{Q}_{3}\right)}-3 G(u)$ over $\mathbf{Q}_{3}$.

- $c_{\pi} \equiv-G(u)^{3} \bmod \left(3, u^{3 e\left(F^{\prime} / \mathbf{Q}_{3}\right)}\right)$.

- For $\gamma \in \operatorname{Gal}\left(F^{\prime} / \mathbf{Q}_{3}\right)$, the unique polynomial $H_{\gamma}(u) \in W\left(k_{F^{\prime}}\right)[u]$ of degree at most $e\left(F^{\prime} / \mathbf{Q}_{3}\right)-1$ such that $\gamma(\pi) / \pi=H_{\gamma}(\pi)$.

- In some cases power series $t_{\gamma}$ and $f_{\gamma, \gamma^{\prime}}$ as in 5.6 .

6.1. The case of $F_{1}^{\prime}$. Recall that $\tau_{1}$ corresponds to the order 3 homomorphism

$$
\mathbf{Z}_{3}^{\times} \longrightarrow \mathrm{GL}_{2}\left(\overline{\mathbf{Q}}_{3}\right)
$$

is determined by

$$
\begin{aligned}
-1 & \longmapsto 1 \\
4 & \longmapsto \zeta,
\end{aligned}
$$

where $\operatorname{det} \zeta=1$ and $\zeta^{3}=1$ but $\zeta \neq 1$. Recall also that $F_{1}^{\prime}=F_{1}$ is any totally ramified cubic Galois extension of $\mathbf{Q}_{3}$. We may take $F_{1}^{\prime}=F_{1}=\mathbf{Q}_{3}[\pi]$, where $\pi$ is a root of $X^{3}-3 X^{2}+3$. One may check that the other roots of $X^{3}-3 X^{2}+3$ are $\pi^{2}-2 \pi$ and $3+\pi-\pi^{2}$, so $\operatorname{Gal}\left(F_{1}^{\prime} / \mathbf{Q}_{3}\right)$ is generated by one element $\gamma_{3}$, which sends 
$\pi$ to $\pi^{2}-2 \pi$ and satisfies $\gamma_{3}^{3}=1$. Also, $\pi$ is a uniformiser for $F_{1}^{\prime}$, so

- $G(u)=u^{2}-1$

- $c_{\pi} \equiv 1-u^{6} \bmod \left(3, u^{9}\right)$,

- $H_{\gamma_{3}}(u)=u-2$.

6.2. The case of $F_{-1}^{\prime}$. Recall that $\tau_{-1}$ corresponds to the order 3 homomorphism

$$
\mathbf{Z}_{3}[\sqrt{-1}]^{\times} \longrightarrow G L_{2}\left(\overline{\mathbf{Q}}_{3}\right)
$$

determined by

$$
\begin{aligned}
\sqrt[4]{-1} & \longmapsto 1 \\
4 & \longmapsto 1 \\
1+3 \sqrt{-1} & \longmapsto \zeta,
\end{aligned}
$$

where $\operatorname{det} \zeta=1$ and $\zeta^{3}=1$ but $\zeta \neq 1$. Recall also that $F_{-1}^{\prime} / \mathbf{Q}_{3}(\sqrt{-1})$ is the unique cubic extension such that $F_{-1}^{\prime} / \mathbf{Q}_{3}$ is Galois but not abelian and that $F_{-1}$ is any cubic subfield. We may take $F_{-1}=\mathbf{Q}_{3}(\pi)$ and $F_{-1}^{\prime}=F_{-1}(\sqrt{-1})$, where $\pi$ is a root of $X^{3}-3 X^{2}+6$. The other roots of $X^{3}-3 X^{2}+6$ are $\left(\sqrt{-1} \pi^{2}-\pi+3(1-\sqrt{-1})\right) / 2$ and $\left(-\sqrt{-1} \pi^{2}-\pi+3(1+\sqrt{-1})\right) / 2$. Thus, $\operatorname{Gal}\left(F_{-1}^{\prime} / \mathbf{Q}_{3}\right)$ is generated by two elements $\gamma_{2}$ and $\gamma_{3}$ defined by

- $\gamma_{2}(\pi)=\pi$,

- $\gamma_{2}(\sqrt{-1})=-\sqrt{-1}$

- $\gamma_{3}(\pi)=\left(\sqrt{-1} \pi^{2}-\pi+3(1-\sqrt{-1})\right) / 2$,

- and $\gamma_{3}(\sqrt{-1})=\sqrt{-1}$.

We have $\gamma_{2}^{2}=\gamma_{3}^{3}=1$ and $\gamma_{2} \gamma_{3}=\gamma_{3}^{2} \gamma_{2}$, and $\pi$ is a uniformiser for $F_{-1}^{\prime}$. Thus

- $G(u)=u^{2}-2$,

- $c_{\pi} \equiv-1-u^{6} \bmod \left(3, u^{9}\right)$,

- $H_{\gamma_{2}}(u)=1$,

- $H_{\gamma_{3}}(u)=\left((\sqrt{-1}-1) u^{2}+(3-\sqrt{-1}) u-2\right) / 4$.

6.3. The case of $F_{3}^{\prime}$. Recall that $\tau_{3}$ is the unique 3-type such that $\left.\tau_{3}\right|_{I_{\mathbf{Q}_{3}(\sqrt{3})}}$ corresponds to the order 6 homomorphism

$$
\mathbf{Z}_{3}[\sqrt{3}]^{\times} \longrightarrow G L_{2}\left(\overline{\mathbf{Q}}_{3}\right)
$$

determined by

$$
\begin{aligned}
-1 & \longmapsto-1 \\
4 & \longmapsto 1 \\
1+\sqrt{3} & \longmapsto \zeta,
\end{aligned}
$$

where $\operatorname{det} \zeta=1$ and $\zeta^{3}=1$ but $\zeta \neq 1$. Recall also that $F_{3}^{\prime}$ is the degree 12 abelian extension of $\mathbf{Q}_{3}(\sqrt{3})$ with norm subgroup in $\mathbf{Q}_{3}(\sqrt{3})^{\times}$topologically generated by 3,4 and $1+3 \sqrt{3}$. We also let $\gamma_{4}^{2}$ denote the unique element of $I_{F_{3}^{\prime} / \mathbf{Q}_{3}(\sqrt{3})}$ of order 3 and we let $F_{3}$ denote the fixed field of some Frobenius lift of order 2 .

We claim that $F_{3}^{\prime}=\mathbf{Q}_{3}(\sqrt{3})(\sqrt{-1}, \alpha, \beta)$, where $\alpha$ is a root of $X^{3}-3 X+3$ and $\beta$ a root of $X^{2}-\sqrt{3}$. To verify this, set $F^{\prime \prime}=\mathbf{Q}_{3}(\sqrt{3})(\sqrt{-1}, \alpha, \beta)$. We must check that $F^{\prime \prime} / \mathbf{Q}_{3}(\sqrt{3})$ is abelian and that $N_{F^{\prime \prime}} / \mathbf{Q}_{3}(\sqrt{3})\left(F^{\prime \prime}\right)^{\times}$contains 3,4 , and $1+3 \sqrt{3}$. To see that $F^{\prime \prime} / \mathbf{Q}_{3}(\sqrt{3})$ is abelian, note that if $\alpha$ is one root of $X^{3}-3 X+3$, then the other roots are $\left(2 \sqrt{3} \alpha^{2}-(-3 \sqrt{3}+\sqrt{-5}) \alpha-4 \sqrt{3}\right) / 2 \sqrt{-5}$ and $\left(-2 \sqrt{3} \alpha^{2}-(3 \sqrt{3}+\sqrt{-5}) \alpha+4 \sqrt{3}\right) / 2 \sqrt{-5}$ (where for definiteness we choose $\left.\sqrt{-5} \in 1+3 \mathbf{Z}_{3}\right)$. Note that $N_{F^{\prime \prime} / \mathbf{Q}_{3}(\sqrt{3})}(\alpha / \beta)=3$ and $N_{F^{\prime \prime} / \mathbf{Q}_{3}(\sqrt{3})}(1+\alpha)=5^{4}$. 
Note that $\operatorname{Gal}\left(F_{3}^{\prime} / \mathbf{Q}_{3}(\sqrt{3})\right)$ is generated by three commuting elements $\gamma_{2}, \gamma_{4}^{2}$ and $\gamma_{3}$ of respective orders 2,2 and 3 . They may be defined by

- $\gamma_{2} \sqrt{-1}=-\sqrt{-1}, \gamma_{2} \beta=\beta$ and $\gamma_{2} \alpha=\alpha$;

- $\gamma_{4}^{2} \sqrt{-1}=\sqrt{-1}, \gamma_{4}^{2} \beta=-\beta$ and $\gamma_{4}^{2} \alpha=\alpha$;

- $\gamma_{3} \sqrt{-1}=\sqrt{-1}, \gamma_{3} \beta=\beta$ and $\gamma_{3} \alpha=\left(-2 \sqrt{3} \alpha^{2}-(3 \sqrt{3}+\sqrt{-5}) \alpha+4 \sqrt{3}\right) / 2 \sqrt{-5}$.

Choose an element $\gamma \in I_{F_{3}^{\prime} / \mathbf{Q}_{3}}-I_{F_{3}^{\prime} / \mathbf{Q}_{3}(\sqrt{3})}$. Then $\gamma^{2} \in\left\langle\gamma_{4}^{2}, \gamma_{3}\right\rangle$. As $\gamma \gamma_{3} \gamma^{-1}=\gamma_{3}^{2}$ we may alter our choice of $\gamma$ so that $\gamma^{2} \in\left\langle\gamma_{4}^{2}\right\rangle$. As $\gamma \sqrt{3}=-\sqrt{3}$ we see that $\gamma \beta= \pm \sqrt{-1} \beta$, so $\gamma^{2}=\gamma_{4}^{2}$. We will rename $\gamma$ as $\gamma_{4}$ and suppose it chosen so that $\gamma_{4} \beta=\sqrt{-1} \beta$. Thus, $\operatorname{Gal}\left(F_{3}^{\prime} / \mathbf{Q}_{3}\right)$ is generated by elements $\gamma_{2}, \gamma_{3}$ and $\gamma_{4}$ satisfying

- $\gamma_{2}^{2}=\gamma_{3}^{3}=\gamma_{4}^{4}=1$,

- $\gamma_{2} \gamma_{3}=\gamma_{3} \gamma_{2}$,

- $\gamma_{4} \gamma_{2}=\gamma_{2} \gamma_{4}^{-1}$,

- and $\gamma_{4} \gamma_{3}=\gamma_{3}^{2} \gamma_{4}$.

The element $\gamma_{4}^{2}$ is the unique element of $I_{F_{3}^{\prime} / \mathbf{Q}_{3}(\sqrt{3})}$ of order 2 and hence coincides with our previous definition. The element $\gamma_{2}$ is a Frobenius lift of order 2 and so we may take $F_{3}$ to be its fixed field, i.e. $F_{3}=\mathbf{Q}_{3}(\pi)$, where $\pi=\alpha / \beta$ is a uniformiser for $F_{3}^{\prime}$. (We are not asserting that $\gamma_{2}$ equals the element denoted $\widetilde{\gamma}_{2}$ in 4 !) One can check that

$$
\gamma_{3}(\pi) / \pi \equiv 1+\pi^{2} \bmod \pi^{4} .
$$

Note also that $\left\langle\gamma_{2}, \gamma_{4}\right\rangle$ projects isomorphically to the quotient of $\operatorname{Gal}\left(F_{3}^{\prime} / \mathbf{Q}_{3}\right)$ by the wild inertia subgroup.

We conclude

- $G(0)=1$,

- $c_{\pi} \equiv-1 \bmod (3, u)$,

- $H_{\gamma_{2}}(u)=1$,

- $H_{\gamma_{4}}(u)=-\sqrt{-1}$,

- $H_{\gamma_{3}}(u) \equiv 1+u^{2} \bmod \left(3, u^{4}\right)$,

- $t_{g}=f_{g, g^{\prime}}=0$ for $g, g^{\prime} \in\left\langle\gamma_{2}, \gamma_{4}\right\rangle$.

6.4. The case of $F_{-3}^{\prime}$. Recall that $\tau_{-3}$ is the unique 3-type such that $\left.\tau_{-3}\right|_{I_{\mathbf{Q}_{3}(\sqrt{ }-3)}}$ corresponds to the order 6 homomorphism

$$
\mathbf{Z}_{3}[\sqrt{-3}]^{\times} \longrightarrow \mathrm{GL}_{2}\left(\overline{\mathbf{Q}}_{3}\right)
$$

determined by

$$
\begin{aligned}
-1 & \longmapsto-1 \\
4 & \longmapsto 1 \\
1+3 \sqrt{-3} & \longmapsto 1 \\
1+\sqrt{-3} & \longmapsto \zeta,
\end{aligned}
$$

where $\operatorname{det} \zeta=1$ and $\zeta^{3}=1$ but $\zeta \neq 1$. Recall also that $F_{-3}^{\prime}$ is the degree 12 abelian extension of $\mathbf{Q}_{3}(\sqrt{-3})$ with norm subgroup in $Q_{3}(\sqrt{-3})^{\times}$topologically generated by $-3,4$ and $1+3 \sqrt{-3}$. We also let $\gamma_{4}^{2}$ denote the unique element of $I_{F_{-3}^{\prime} / \mathbf{Q}_{3}(\sqrt{-3})}$ of order 3 and we let $F_{-3}$ denote the fixed field of some Frobenius lift of order 2 .

We claim that $F_{-3}^{\prime}=\mathbf{Q}_{3}(\sqrt{-3})(\sqrt{-1}, \alpha, \beta)$ where $\alpha$ is a root of $X^{3}-4$ and $\beta$ a root of $X^{2}+\sqrt{-3}$. To verify this, set $F^{\prime \prime}=\mathbf{Q}_{3}(\sqrt{-3})(\sqrt{-1}, \alpha, \beta)$. Then $F^{\prime \prime} / \mathbf{Q}_{3}(\sqrt{-3})$ is abelian and so we must check that $N_{F^{\prime \prime}} / \mathbf{Q}_{3}(\sqrt{-3})\left(F^{\prime \prime}\right)^{\times}$contains -3 , 
4 and $1+3 \sqrt{-3}$. But note that we have the identities $N_{F^{\prime \prime} / \mathbf{Q}_{3}(\sqrt{-3})}((\alpha-1) / \beta)=-3$, $N_{F^{\prime \prime} / \mathbf{Q}_{3}(\sqrt{-3})}(\alpha)=4^{4}$ and $N_{F^{\prime \prime} / \mathbf{Q}_{3}(\sqrt{-3})}(1-\beta)=(1+\sqrt{-3})^{6}$.

Note that $\operatorname{Gal}\left(F_{3}^{\prime} / \mathbf{Q}_{3}(\sqrt{-3})\right)$ is generated by three commuting elements $\gamma_{2}, \gamma_{4}^{2}$ and $\gamma_{3}$ of respective orders 2,2 and 3 . They may be defined by

- $\gamma_{2} \sqrt{-1}=-\sqrt{-1}, \gamma_{2} \beta=\beta$ and $\gamma_{2} \alpha=\alpha$;

- $\gamma_{4}^{2} \sqrt{-1}=\sqrt{-1}, \gamma_{4}^{2} \beta=-\beta$ and $\gamma_{4}^{2} \alpha=\alpha$;

- $\gamma_{3} \sqrt{-1}=\sqrt{-1}, \gamma_{3} \beta=\beta$ and $\gamma_{3} \alpha=(-1-\sqrt{-3}) \alpha / 2$.

Choose an element $\gamma \in I_{F_{-3}^{\prime} / \mathbf{Q}_{3}}-I_{F_{-3}^{\prime} / \mathbf{Q}_{3}(\sqrt{-3})}$, so $\gamma^{2} \in\left\langle\gamma_{4}^{2}, \gamma_{3}\right\rangle$. As $\gamma \gamma_{3} \gamma^{-1}=\gamma_{3}^{2}$, we may alter our choice of $\gamma$ so that $\gamma^{2} \in\left\langle\gamma_{4}^{2}\right\rangle$. As $\gamma \sqrt{-3}=-\sqrt{-3}$ we see that $\gamma \beta= \pm \sqrt{-1} \beta$, so $\gamma^{2}=\gamma_{4}^{2}$. We will rename $\gamma$ as $\gamma_{4}$ and suppose it chosen so that $\gamma_{4} \beta=\sqrt{-1} \beta$. Thus, $\operatorname{Gal}\left(F_{3}^{\prime} / \mathbf{Q}_{3}\right)$ is generated by elements $\gamma_{2}, \gamma_{3}$ and $\gamma_{4}$ satisfying

- $\gamma_{2}^{2}=\gamma_{3}^{3}=\gamma_{4}^{4}=1$,

- $\gamma_{2} \gamma_{3}=\gamma_{3} \gamma_{2}$,

- $\gamma_{4} \gamma_{2}=\gamma_{2} \gamma_{4}^{-1}$,

- and $\gamma_{4} \gamma_{3}=\gamma_{3}^{2} \gamma_{4}$.

The element $\gamma_{4}^{2}$ is the unique element of $I_{F_{-3}^{\prime} / \mathbf{Q}_{3}(\sqrt{-3})}$ of order 2 and hence coincides with our previous definition. The element $\gamma_{2}$ is a Frobenius lift of order 2 and so we may take $F_{-3}$ to be its fixed field, i.e. $F_{-3}=\mathbf{Q}_{3}(\pi)$, where $\pi=\alpha / \beta$ is a uniformiser for $F_{-3}^{\prime}$. (We are not asserting that $\gamma_{2}$ equals the element denoted $\widetilde{\gamma}_{2}$ in 4 ) One can check that

$$
\gamma_{3}(\pi) / \pi \equiv 1+\pi^{2} \bmod \pi^{4} .
$$

Note also that $\left\langle\gamma_{2}, \gamma_{4}\right\rangle$ lifts tame inertia.

We conclude

- $G(0)=-1$,

- $c_{\pi} \equiv 1 \bmod (3, u)$,

- $H_{\gamma_{2}}(u)=1$,

- $H_{\gamma_{4}}(u)=-\sqrt{-1}$,

- $H_{\gamma_{3}}(u) \equiv 1+u^{2} \bmod \left(3, u^{4}\right)$

- $t_{g}=f_{g, g^{\prime}}=0$ for $g, g^{\prime} \in\left\langle\gamma_{2}, \gamma_{4}\right\rangle$.

6.5. The case of $F_{i}^{\prime}$. Here $i \in \mathbf{Z} / 3 \mathbf{Z}$ and we will let $\tilde{\imath}$ denote the unique lifting of $i$ to $\mathbf{Z}$ with $0 \leq \tilde{\imath}<3$. Recall that $\tau_{i}^{\prime}$ is the unique extended 3 -type whose restrictions to $G_{\mathbf{Q}_{3}(\sqrt{-3})}$ correspond to the homomorphism

$$
\mathbf{Q}_{3}(\sqrt{-3})^{\times} \longrightarrow \mathrm{GL}_{2}\left(\overline{\mathbf{Q}}_{3}\right)
$$

determined by

$$
\begin{aligned}
\sqrt{-3} & \longmapsto \zeta-\zeta^{-1} \\
-1 & \longmapsto-1 \\
4 & \longmapsto 1 \\
1+3 \sqrt{-3} & \longmapsto \zeta \\
1+\sqrt{-3} & \longmapsto \zeta^{i},
\end{aligned}
$$

where $\operatorname{det} \zeta=1$ and $\zeta^{3}=1$ but $\zeta \neq 1$. Recall also that $F_{i}^{\prime}$ is the degree 12 abelian extension of $\mathbf{Q}_{3}(\sqrt{-3})$ with norms the subgroup of $\mathbf{Q}_{3}(\sqrt{-3})^{\times}$topologically generated by $-3,4,1+9 \sqrt{-3}$ and $1+(1-3 \tilde{\imath}) \sqrt{-3}$. We let $\gamma_{2}, \gamma_{3}$ and $\gamma_{4}^{2}$ denote the elements of $\operatorname{Gal}\left(F_{i}^{\prime} / \mathbf{Q}_{3}\right)$ which correspond respectively to $\sqrt{-3}, 1-3 \sqrt{-3}$ and -1 . 
We claim that $F_{i}^{\prime}=\mathbf{Q}_{3}(\sqrt{-3})(\sqrt{-1}, \alpha, \beta)$, where $\alpha$ is a root of $X^{3}-3(1+3 \tilde{\imath})$ and $\beta$ a root of $X^{2}+\sqrt{-3}$. To verify this, set $F^{\prime \prime}=\mathbf{Q}_{3}(\sqrt{-3})(\sqrt{-1}, \alpha, \beta)$, so $F^{\prime \prime} / \mathbf{Q}_{3}(\sqrt{-3})$ is abelian and we must check that $N_{F^{\prime \prime} / \mathbf{Q}_{3}(\sqrt{-3})}\left(F^{\prime \prime}\right)^{\times}$contains -3 , $4,1+9 \sqrt{-3}$, and $1+(1-3 \tilde{\imath}) \sqrt{-3}$. But note that $N_{F^{\prime \prime} / \mathbf{Q}_{3}(\sqrt{-3})}(\alpha / \beta)=-3(1+3 \tilde{\imath})^{4}$, $N_{F^{\prime \prime} / \mathbf{Q}_{3}(\sqrt{-3})}(1+\alpha)=(4+9 \tilde{\imath})^{4}$ and

$$
\begin{aligned}
N_{F^{\prime \prime} / \mathbf{Q}_{3}(\sqrt{-3})}(\beta(\sqrt{-3}-\alpha) / \alpha) & =(1+\sqrt{-3}+3 \tilde{\imath}) /(1+3 \tilde{\imath})^{4} \\
& \equiv 1+(1-3 \tilde{\imath}) \sqrt{-3} \bmod 9 .
\end{aligned}
$$

Note that $\gamma_{4}^{2}$ is an element of $I_{F_{i}^{\prime} / \mathbf{Q}_{3}(\sqrt{-3})}$ of order $2, \gamma_{2} \neq \gamma_{4}^{2}$ but also has order 2 , and $\gamma_{3}$ is an element of $I_{F_{i}^{\prime} / \mathbf{Q}_{3}(\sqrt{-3})}$ of order 3. Thus,

- $\gamma_{4}^{2} \sqrt{-1}=\sqrt{-1}, \gamma_{4}^{2} \beta=-\beta$ and $\gamma_{4}^{2} \alpha=\alpha$

- $\gamma_{2} \sqrt{-1}=-\sqrt{-1}$ and $\gamma_{2} \alpha=\alpha$;

- $\gamma_{3} \sqrt{-1}=\sqrt{-1}$ and $\gamma_{3} \beta=\beta$.

Moreover $\sqrt{-3}$ is a norm from $\mathbf{Q}_{3}(\sqrt{-3})(\alpha, \beta)$, because $\alpha / \beta$ has norm $\sqrt{-3}(1+3 \tilde{\imath})^{2}$, so

- $\gamma_{2}(\beta)=\beta$.

The determination of $\gamma_{3}(\alpha)$ is more delicate. Let $\delta$ be a root of $X^{3}-$ $(1+3 \sqrt{-3})$, so $\delta=1+\sqrt{-3} \mu$, where $\mu$ is a root of $Y^{3}-\sqrt{-3} Y^{2}-Y+1$. Thus $\mathbf{Q}_{3}(\sqrt{-3})(\delta) / \mathbf{Q}_{3}(\sqrt{-3})$ is unramified and

$$
\operatorname{Frob}_{3}(\delta) / \delta \equiv\left(1+\sqrt{-3} \mu^{3}\right) /(1+\sqrt{-3} \mu) \equiv(-1+\sqrt{-3}) / 2 \bmod 3 .
$$

The norms from $\mathbf{Q}_{3}(\sqrt{-3})(\delta)^{\times}$to $\mathbf{Q}_{3}(\sqrt{-3})^{\times}$are generated by $\mathbf{Z}_{3}[\sqrt{-3}]^{\times}$and $3 \sqrt{-3}$. The norms from $\mathbf{Q}_{3}(\sqrt{-3})(\alpha)^{\times}$to $\mathbf{Q}_{3}(\sqrt{-3})^{\times}$are generated by $1+9 \mathbf{Z}_{3}[\sqrt{-3}]$, $1+(1-3 \tilde{\imath}) \sqrt{-3}, 4,-1$ and $\sqrt{-3}$. The norms from $\mathbf{Q}_{3}(\sqrt{-3})(\alpha, \delta)^{\times}$to $\mathbf{Q}_{3}(\sqrt{-3})^{\times}$ are generated by $1+9 \mathbf{Z}_{3}[\sqrt{-3}], 1+(1-3 \tilde{\imath}) \sqrt{-3}, 4,-1$ and $3 \sqrt{-3}$. Thus

$$
\begin{aligned}
\left(\gamma_{3}, \operatorname{Frob}_{3}\right) & \in \operatorname{Gal}\left(\mathbf{Q}_{3}(\sqrt{-3})(\alpha) / \mathbf{Q}_{3}(\sqrt{-3})\right) \times \operatorname{Gal}\left(\mathbf{Q}_{3}(\sqrt{-3})(\delta) / \mathbf{Q}_{3}(\sqrt{-3})\right) \\
& \cong \operatorname{Gal}\left(\mathbf{Q}_{3}(\sqrt{-3})(\alpha, \delta) / \mathbf{Q}_{3}(\sqrt{-3})\right)
\end{aligned}
$$

corresponds to $\sqrt{-3}(1-3 \sqrt{-3}) \in \mathbf{Q}_{3}(\sqrt{-3})^{\times}$. As $\delta \alpha$ has norm to $\mathbf{Q}_{3}(\sqrt{-3})$ the product of $(\sqrt{-3}(1-3 \sqrt{-3}))^{2}$ and $-(1+3 \tilde{\imath})(1+3 \sqrt{-3}) /(1-3 \sqrt{-3})^{2}$, we conclude that $\left(\gamma_{3}\right.$, Frob $\left._{3}\right)$ fixes $\delta \alpha$. Thus $\gamma_{3}(\alpha) / \alpha=\delta / \operatorname{Frob}_{3}(\delta)=(-1-\sqrt{-3}) / 2$. In other words

- $\gamma_{3}(\alpha)=(-1-\sqrt{-3}) \alpha / 2$.

Choose an element $\gamma \in I_{F_{i}^{\prime} / \mathbf{Q}_{3}}-I_{F_{i}^{\prime} / \mathbf{Q}_{3}(\sqrt{-3})}$. Then $\gamma^{2} \in\left\langle\gamma_{4}^{2}, \gamma_{3}\right\rangle$. As $\gamma \gamma_{3} \gamma^{-1}=$ $\gamma_{3}^{2}$ we may alter our choice of $\gamma$ so that $\gamma^{2} \in\left\langle\gamma_{4}^{2}\right\rangle$. As $\gamma \sqrt{-3}=-\sqrt{-3}$ we see that $\gamma \beta= \pm \sqrt{-1} \beta$ and so $\gamma^{2}=\gamma_{4}^{2}$. We will rename $\gamma$ as $\gamma_{4}$ and suppose it chosen so that $\gamma_{4} \beta=\sqrt{-1} \beta$. Thus, $\operatorname{Gal}\left(F_{i}^{\prime} / \mathbf{Q}_{3}\right)$ is generated by elements $\gamma_{2}, \gamma_{3}$ and $\gamma_{4}$ satisfying

- $\gamma_{2}^{2}=\gamma_{3}^{3}=\gamma_{4}^{4}=1$,

- $\gamma_{2} \gamma_{3}=\gamma_{3} \gamma_{2}$

- $\gamma_{4} \gamma_{2}=\gamma_{2} \gamma_{4}^{-1}$,

- and $\gamma_{4} \gamma_{3}=\gamma_{3}^{2} \gamma_{4}$.

The element $\gamma_{2}$ is a Frobenius lift and it has fixed field $F_{i}=\mathbf{Q}_{3}(\pi)$, where $\pi=\alpha / \beta$ is a uniformiser for $F_{i}^{\prime}$. One can check that

$$
\gamma_{3}^{ \pm 1}(\pi) / \pi=-\left(1 \mp(1+3 \tilde{\imath})^{-2} \pi^{6}\right) / 2 .
$$


We conclude

- $G(u)=-(1+3 \tilde{\imath})^{4}$

- $c_{\pi} \equiv 1 \bmod \left(3, u^{36}\right)$,

- $H_{\gamma_{2}}(u)=1$,

- $H_{\gamma_{4}}(u)=-\sqrt{-1}$,

- $H_{\gamma_{3}^{ \pm 1}}(u) \equiv 1 \mp u^{6} \bmod 3$,

- $t_{\gamma_{3}^{ \pm 1}}(u) \equiv-1 \mp u^{6} \bmod \left(3, u^{12}\right)$,

- $t_{g}=f_{g, g^{\prime}}=0$ for $g, g^{\prime} \in\left\langle\gamma_{2}, \gamma_{4}\right\rangle$,

- $f_{\gamma_{3}^{ \pm 1}, \gamma_{3}^{ \pm 1}}^{ \pm}(u), f_{\gamma_{3}^{ \pm 1}, \gamma_{3}^{\mp 1}}^{\mp}(u) \equiv 0 \bmod \left(3, u^{12}\right)$.

\section{Proof of Theorem 4.4.1}

In this section we will keep the notation of 44.4 and either $\$ 6.1$ or $\$ 6.2$ (depending on whether we are working with $\mathcal{S}_{1}$ or $\mathcal{S}_{-1}$ ). We will set $\delta= \pm 1$ in the case of $\mathcal{S}_{ \pm 1}$. We will write $F$ for $F_{ \pm 1}$ and $F^{\prime}$ for $F_{ \pm 1}^{\prime}$. If $\mathcal{G}$ (resp. $\mathcal{M}$ ) is a finite flat $\mathcal{O}_{F}$-group scheme (resp. Breuil module over $\mathcal{O}_{F}$ ) we will write $\mathcal{G}^{\prime}\left(\right.$ resp. $\mathcal{M}^{\prime}$ ) for the unramified base change to $\mathcal{O}_{F^{\prime}}$.

7.1. Rank one calculations. We recall from Lemma 5.2.1 that the only $\mathcal{O}_{F^{-}}$ models for $(\mathbf{Z} / 3 \mathbf{Z})_{/ F}$ are $\mathcal{G}(3, \delta) \cong(\mathbf{Z} / 3 \mathbf{Z})_{/ \mathcal{O}_{F}}$ and $\mathcal{G}(1, \delta)$, and the only $\mathcal{O}_{F}$-models for $\left(\mu_{3}\right)_{/ F}$ are $\mathcal{G}(0,1) \cong\left(\mu_{3}\right) / \mathcal{O}_{F}$ and $\mathcal{G}(2,1)$. In each case, by Lemma 5.7.1, the base change to $\mathcal{O}_{F^{\prime}}$ admits unique descent data over $\mathbf{Q}_{3}$ compatible with the canonical descent data on the generic fibre of $\mathbf{Z} / 3 \mathbf{Z}$ (resp. $\mu_{3}$ ) over $\mathbf{Q}_{3}$. We will refer to this descent data as the standard descent data on these finite flat group schemes.

\subsection{Rank two calculations.}

Lemma 7.2.1. The group of extensions of $\mathcal{M}(2,1)$ by $\mathcal{M}(1, \delta)$ over $\mathcal{O}_{F}$ is parametrised by $c \in \mathbf{F}_{3}$. The Breuil module $\mathcal{M}(1, \delta ; 2,1 ; c)$ corresponding to $c$ is free of rank two over $\mathbf{F}_{3}[u] / u^{9}$ with a basis $\left\{\mathbf{e}_{1}, \mathbf{e}_{\omega}\right\}$ such that

- $\mathcal{M}_{1}=\left\langle u \mathbf{e}_{1}, u^{2} \mathbf{e}_{\omega}+c \mathbf{e}_{1}\right\rangle$,

- $\phi_{1}\left(u \mathbf{e}_{1}\right)=\delta \mathbf{e}_{1}, \phi_{1}\left(u^{2} \mathbf{e}_{\omega}+c \mathbf{e}_{1}\right)=\mathbf{e}_{\omega}$,

- $N\left(\mathbf{e}_{1}\right)=0, N\left(\mathbf{e}_{\omega}\right)=c u^{6} \mathbf{e}_{1}$.

The standard descent data on $\mathcal{M}(2,1)^{\prime}$ and $\mathcal{M}(1, \delta)^{\prime}$ extends uniquely to descent data on $\mathcal{N}(1, \delta ; 2,1 ; c)^{\prime}$. The corresponding representations $G_{3} \rightarrow \mathrm{GL}_{2}\left(\mathbf{F}_{3}\right)$ are of the form

$$
\left(\begin{array}{cc}
\omega & * \\
0 & 1
\end{array}\right)
$$

and are peu ramifié. Any such peu-ramifié extension arises for a suitable choice of $c$.

Proof. The classification of extensions of Breuil modules follows from Lemma 5.2.2 Next, we compute $N$ on $\mathcal{M}=\mathcal{M}(1, \delta ; 2,1 ; c)$. (We will not in fact need the result of this computation of $N$, but the calculation is given here as a representative sample of calculations needed later in more complicated settings.) By the last part of Lemma 5.2.1 $N\left(\mathbf{e}_{1}\right)=0$ and $N\left(\mathbf{e}_{\omega}\right)=g \mathbf{e}_{1}$ for some $g \in \mathbf{F}_{3}[u] / u^{e \ell}$ divisible by $u$. In $\mathbf{F}_{3}[u] / u^{9}$ we compute

$$
c_{\pi}=-\phi\left(G_{\pi}(u)\right)=-\left(u^{2}-\delta\right)^{3}=-u^{6}+\delta,
$$


So

$$
\frac{\delta u^{6}}{c_{\pi}}=u^{6}
$$

Using the defining properties of $N$, we compute in $\mathbf{F}_{3}[u] / u^{9}$

$$
\begin{aligned}
N\left(\mathbf{e}_{\omega}\right) & =N \circ \phi_{1}\left(u^{2} \mathbf{e}_{\omega}+c \mathbf{e}_{1}\right) \\
& =\phi \circ N\left(u^{2} \mathbf{e}_{\omega}+c \mathbf{e}_{1}\right) \\
& =\phi\left(-u^{2} \mathbf{e}_{\omega}+u^{2} N\left(\mathbf{e}_{\omega}\right)\right) \\
& =\frac{\phi_{1}}{c_{\pi}}\left(-u^{5} \mathbf{e}_{\omega}+u^{5} N\left(\mathbf{e}_{\omega}\right)\right) \\
& =\frac{\phi_{1}}{c_{\pi}}\left(-u^{3}\left(u^{2} \mathbf{e}_{\omega}+c \mathbf{e}_{1}\right)+c u^{3} \mathbf{e}_{1}+u^{5} N\left(\mathbf{e}_{\omega}\right)\right) \\
& =\frac{\phi_{1}}{c_{\pi}}\left(c u^{3} \mathbf{e}_{1}\right)
\end{aligned}
$$

since $u^{5} N\left(\mathbf{e}_{\omega}\right) \in u^{6} \mathcal{M}=u^{3} \cdot u^{3} \mathcal{M} \subseteq u^{3} \mathcal{M}_{1}$ and the Frobenius-semi-linear $\phi_{1}$ must kill $u^{3} \mathcal{M}_{1}$. Thus,

$$
N\left(\mathbf{e}_{\omega}\right)=\frac{\phi_{1}}{c_{\pi}}\left(c u^{2} \cdot u \mathbf{e}_{1}\right)=\frac{c u^{6}}{c_{\pi}} \phi_{1}\left(u \mathbf{e}_{1}\right)=\frac{c \delta u^{6}}{c_{\pi}} \mathbf{e}_{1}=c u^{6} \mathbf{e}_{1} .
$$

To see existence and uniqueness of the descent data on $\mathcal{M}(1, \delta ; 2,1 ; c)^{\prime}$ compatible with the standard descent data on $\mathcal{M}(1, \delta)^{\prime}$ and $\mathcal{M}(2,1)^{\prime}$ we will work on the side of finite flat group schemes. Because $\mathcal{G}(1, \delta ; 2,1 ; c)^{\prime}$ is the unique extension of $\mathcal{G}(1, \delta)^{\prime}$ by $\mathcal{G}(2,1)^{\prime}$ with generic fibre $\mathcal{G}(1, \delta ; 2,1 ; c)^{\prime} \times F^{\prime}$ (by Lemma 4.1.2), uniqueness reduces to the corresponding questions on the generic fibre, which follows from the injectivity of

$$
H^{1}\left(G_{3}, \omega\right) \longrightarrow H^{1}\left(G_{F^{\prime}}, \omega\right)
$$

For existence it suffices to exhibit a continuous representation $G_{3} \rightarrow \mathrm{GL}_{2}\left(\mathbf{F}_{3}\right)$ of the form

$$
\left(\begin{array}{ll}
\omega & * \\
0 & 1
\end{array}\right)
$$

which is peu ramifié but not split, with restriction to $G_{F}$ corresponding to a locallocal finite flat $\mathcal{O}_{F}$-group scheme $\mathcal{G}$. By Theorem 5.3.2 of [Man] we can find an elliptic curve $E_{/ \mathbf{Q}_{3}}$ such that $E[3]$ furnishes the desired example. This also proves the final two assertions of the lemma.

Lemma 7.2.2. Suppose that $\widetilde{F}_{1}$ is a totally ramified abelian cubic extension of $\mathbf{Q}_{3}$ and suppose that $\mathcal{G}$ is a local-local finite flat $\mathcal{O}_{\widetilde{F}_{1}}$-group scheme killed by 3 such that $\mathcal{G} \times \widetilde{F}_{1}$ is an extension of $\mathbf{Z} / 3 \mathbf{Z}$ by $\mu_{3}$. Then $\mathcal{G} \times{ }_{\mathcal{O}_{F_{1}}} \widetilde{F}_{1} \cong G \times{ }_{\mathbf{Q}_{3}} \widetilde{F}_{1}$ for some finite flat $\mathbf{Q}_{3}$-group scheme $G$.

Proof. As in the proof of the last lemma we see that $\mathcal{M}_{\pi}(\mathcal{G}) \cong \mathcal{M}(1,1 ; 2,1 ; c)$ for some $c \in \mathbf{F}_{3}$. As the only action of $\operatorname{Gal}\left(\widetilde{F}_{1} / \mathbf{Q}_{3}\right)$ on a one-dimensional $\mathbf{F}_{3 \text {-vector }}$ space is trivial, we see that each such $c$ gives a class in $H^{1}\left(G_{\widetilde{F}_{1}}, \omega\right)$ which is invariant by $\operatorname{Gal}\left(\widetilde{F}_{1} / \mathbf{Q}_{3}\right)$. But

$$
H^{1}\left(G_{3}, \omega\right) \stackrel{\sim}{\longrightarrow} H^{1}\left(G_{\widetilde{F}_{1}}, \omega\right)^{\operatorname{Gal}\left(\widetilde{F}_{1} / \mathbf{Q}_{3}\right)},
$$

and so the lemma follows. 
Lemma 7.2.3. The group of extensions of $\mathcal{M}(1, \delta)$ by $\mathcal{M}(2,1)$ over $\mathcal{O}_{F}$ is isomorphic to the group of linear polynomials $c+c^{\prime} u$ in $\mathbf{F}_{3}[u]$. The Breuil module $\mathcal{M}\left(2,1 ; 1, \delta ; c+c^{\prime} u\right)$ corresponding to $c+c^{\prime} u$ is free of rank two over $\mathbf{F}_{3}[u] / u^{9}$ with a basis $\left\{\mathbf{e}_{\omega}, \mathbf{e}_{1}\right\}$ such that

- $\mathcal{M}\left(2,1 ; 1, \delta ; c+c^{\prime} u\right)_{1}=\left\langle u^{2} \mathbf{e}_{\omega}, u \mathbf{e}_{1}+\left(c+c^{\prime} u\right) \mathbf{e}_{\omega}\right\rangle$,

- $\phi_{1}\left(u^{2} \mathbf{e}_{\omega}\right)=\mathbf{e}_{\omega}, \phi_{1}\left(u \mathbf{e}_{1}+\left(c+c^{\prime} u\right) \mathbf{e}_{\omega}\right)=\delta \mathbf{e}_{1}$.

Each $\mathcal{M}\left(2,1 ; 1, \delta ; c+c^{\prime} u\right)^{\prime}$ admits unique descent data compatible with the standard descent data on $\mathcal{M}(1, \delta)^{\prime}$ and $\mathcal{M}(2,1)^{\prime}$. As c, $c^{\prime}$ vary over $\mathbf{F}_{3}$ the corresponding descent to $\mathbf{Q}_{3}$ of the generic fibre of $\mathcal{G}_{\pi}\left(\mathcal{M}\left(2,1 ; 1, \delta ; c+c^{\prime} u\right)^{\prime}\right)$ runs over all 9 extensions of $\mu_{3}$ by $\mathbf{Z} / 3 \mathbf{Z}$. The corresponding representation of $G_{3}$ is peu ramifié if and only if $c=0$.

Proof. The classification of extensions of Breuil modules follows from Lemma 5.2 .2 The uniqueness of the descent data on $\mathcal{M}\left(2,1 ; 1, \delta ; c+c^{\prime} u\right)^{\prime}$ follows from Lemma 4.1 .2 and the injectivity of $H^{1}\left(G_{3}, \omega^{-1}\right) \rightarrow H^{1}\left(G_{F^{\prime}}, \omega^{-1}\right)$ as in the proof of Lemma 7.2.1. Note that Frobenius vanishes on the Dieudonné module of $\mathcal{G}\left(2,1 ; 1, \delta ; c+c^{\prime} u\right)$ if and only if $c=0$. Thus the lemma will follow if for each 3-torsion extension $G$ of $\mu_{3}$ by $\mathbf{Z} / 3 \mathbf{Z}$ over $\mathbf{Q}_{3}$ which is très ramifié, we can find a finite flat $\mathcal{O}_{F}$-group scheme $\mathcal{G}$ such that

- the generic fibre of $\mathcal{G}$ is isomorphic to $G \times F$,

- the closed fibre of $\mathcal{G}$ is local-local,

- and Frobenius is not identically zero on $D(\mathcal{G})$.

The splitting field of $G$ contains a cube root of $3 v$ for some $v \equiv 1 \bmod 3$, where the three choices of $v \bmod 9$ correspond to the three choices of très ramifié $\bar{\rho}$. The calculations in $\S 5.3$ of $\mathrm{Man}$ give explicit additive reduction elliptic curves $E$ and $E^{\prime}$ over $\mathbf{Q}_{3}$ with $E[3] \simeq E^{\prime}[3] \simeq G$, where $E$ acquires good supersingular reduction over the non-Galois cubic ramified extension

$$
\mathbf{Q}_{3}[X] /\left(X^{3}-3 X+2 b\right),
$$

with $b^{2}=1+3 v$, and $E^{\prime}$ acquires good supersingular reduction over the abelian cubic ramified extension of $\mathbf{Q}_{3}$ with norm group generated by $3 v \bmod \left(\mathbf{Q}_{3}^{\times}\right)^{3}$. The appropriate $\mathcal{G}$ are provided by the 3 -torsion on the Néron models of $E$ or $E^{\prime}$ over $\mathcal{O}_{F}$.

Corollary 7.2.4. Suppose that $\mathcal{G}$ is a finite flat $\mathcal{O}_{F}$-group scheme and that $\{[g]\}$ is descent data on $\mathcal{G}^{\prime}=\mathcal{G} \times \mathcal{O}_{F^{\prime}}$ such that $\left(\mathcal{G}^{\prime},\{[g]\}\right)_{\mathbf{Q}_{3}}\left(\overline{\mathbf{Q}}_{3}\right)$ corresponds to $\bar{\rho}$. Then

$$
\mathcal{M}_{\pi}(\mathcal{G}) \cong \mathcal{M}\left(2,1 ; 1, \delta ; c+c^{\prime} u\right)
$$

for some $c, c^{\prime} \in \mathbf{F}_{3}$ with $c \neq 0$.

Proof. From the connected-étale exact sequence and its dual we see that $\mathcal{G} \times \mathbf{F}_{3}$ must be local-local. The corollary now follows from Lemma [7.2.3 and the discussion of 7.1

Lemma 7.2.5. The group of extensions of $\mathcal{M}(1, \delta)$ by $\mathcal{M}(1, \delta)$ over $\mathcal{O}_{F}$ is isomorphic to the group of linear polynomials $b+b^{\prime} u$ in $\mathbf{F}_{3}[u]$. The Breuil module $\mathcal{M}\left(1, \delta ; 1, \delta ; b+b^{\prime} u\right)$ corresponding to $b+b^{\prime} u$ is free of rank two over $\mathbf{F}_{3}[u] / u^{9}$ with a basis $\left\{\mathbf{e}, \mathbf{e}^{\prime}\right\}$ such that

- $\mathcal{M}\left(1, \delta ; 1, \delta ; b+b^{\prime} u\right)_{1}=\left\langle u \mathbf{e}, u \mathbf{e}^{\prime}+\left(b+b^{\prime} u\right) \mathbf{e}\right\rangle$,

- $\phi_{1}(u \mathbf{e})=\delta \mathbf{e}, \phi_{1}\left(u \mathbf{e}^{\prime}+\left(b+b^{\prime} u\right) \mathbf{e}\right)=\delta \mathbf{e}^{\prime}$. 
This extension splits over an unramified extension if and only if $b=0$. If $F^{\prime} / \mathbf{Q}_{3}$ is non-abelian, then any descent data on $\mathcal{M}\left(1,-1 ; 1,-1 ; b+b^{\prime} u\right)^{\prime}$ compatible with the standard descent data on $\mathcal{M}(1,-1)^{\prime}$ satisfies

$$
\widehat{\gamma}_{2} \mathbf{e}=\mathbf{e}, \widehat{\gamma}_{2} \mathbf{e}^{\prime}=\mathbf{e}^{\prime}, \widehat{\gamma_{3}^{ \pm 1}}(\mathbf{e})=H_{\gamma_{3}^{ \pm 1}}(u)^{3} \mathbf{e}, \widehat{\gamma_{3}^{ \pm 1}}\left(\mathbf{e}^{\prime}\right)=H_{\gamma_{3}^{ \pm 1}}(u)^{3} \mathbf{e}^{\prime}+h_{\gamma_{3}^{ \pm 1}}(u) \mathbf{e},
$$

where

$$
h_{\gamma_{3}^{ \pm 1}}(0)=-b H_{\gamma_{3}^{ \pm 1}}^{\prime}(0) .
$$

Proof. The classification of extensions of Breuil modules follows from Lemma 5.2.2 The computation of which of these split over an unramified extension follows from Lemma 5.2.2 and Corollary 5.4.2.

Now suppose that $F^{\prime} / \mathbf{Q}_{3}$ is non-abelian. By Lemma 5.7.1 the only issue is to compute $h_{\gamma_{3}}(0)$. Since $H_{\gamma_{3}}(0) \equiv 1 \bmod 3$, by evaluating the congruence

$$
\widehat{\gamma_{3}} \circ \phi_{1}^{\prime} \equiv \phi_{1}^{\prime} \circ \widehat{\gamma_{3}} \bmod u \mathcal{M}\left(1,-1 ; 1,-1 ; b+b^{\prime} u\right)^{\prime}
$$

on $u \mathbf{e}^{\prime}+\left(b+b^{\prime} u\right) \mathbf{e}$ and comparing constant terms of the coefficients of $\mathbf{e}$ on both sides we get

$$
\begin{aligned}
h_{\gamma_{3}}(0) & =h_{\gamma_{3}}(0)^{3}+\left.b\left(\frac{1-H_{\gamma_{3}}(u)}{u}\right)^{3}\right|_{u=0} \\
& =h_{\gamma_{3}}(0)^{3}-b H_{\gamma_{3}}^{\prime}(0)^{3}=h_{\gamma_{3}}(0)^{3}+b H_{\gamma_{3}}^{\prime}(0)
\end{aligned}
$$

in $\mathbf{F}_{9}$, where we have used the equality $H_{\gamma_{3}}^{\prime}(0)^{2} \equiv-1 \bmod 3($ see $\$ 6.2)$ ).

In other words $h_{\gamma_{3}}(0)$ is a root of $T^{3}-T+b H_{\gamma_{3}}^{\prime}(0)=0$. Since $H_{\gamma_{3}}^{\prime}(0)^{2}=-1$, we must have $h_{\gamma_{3}}(0)=-b H_{\gamma_{3}}^{\prime}(0)+a$ for some $a \in \mathbf{F}_{3}$. Since $\gamma_{2}\left(H_{\gamma_{3}}(u)\right)=H_{\gamma_{3}^{-1}}(u)$ and $\gamma_{2}\left(h_{\gamma_{3}}(u)\right)=h_{\gamma_{3}^{-1}}(u)$ are forced by the identity $\gamma_{2}(\pi)=\pi$, we see that $h_{\gamma_{3}^{-1}}(0)=$ $-b H_{\gamma_{3}^{-1}}^{\prime}(0)+a$ for the same $a \in \mathbf{F}_{3}$. The identity

$$
\widehat{\gamma_{3}} \circ \widehat{\gamma_{3}^{-1}} \circ \phi_{1}^{\prime} \equiv \phi_{1}^{\prime} \bmod u \mathcal{M}\left(1,-1 ; 1,-1 ; b+b^{\prime} u\right)^{\prime}
$$

then implies $h_{\gamma_{3}}(0)+h_{\gamma_{3}^{-1}}(0)=0$, so $a=0$.

Lemma 7.2.6. The group of extensions of $\mathcal{M}(2,1)$ by $\mathcal{M}(2,1)$ over $\mathcal{O}_{F}$ is isomorphic to the group of quadratic polynomials vanishing at $0,\left(b+b^{\prime} u\right) u$, in $\mathbf{F}_{3}[u]$. The Breuil module $\mathcal{M}\left(2,1 ; 2,1 ;\left(b+b^{\prime} u\right) u\right)$ corresponding to $\left(b+b^{\prime} u\right) u$ is free of rank two over $\mathbf{F}_{3}[u] / u^{9}$ with a basis $\left\{\mathbf{e}, \mathbf{e}^{\prime}\right\}$ such that

- $\mathcal{M}\left(2,1 ; 2,1 ;\left(b+b^{\prime} u\right) u\right)_{1}=\left\langle u^{2} \mathbf{e}, u^{2} \mathbf{e}^{\prime}+\left(b+b^{\prime} u\right) u \mathbf{e}\right\rangle$,

- $\phi_{1}\left(u^{2} \mathbf{e}\right)=\mathbf{e}, \phi_{1}\left(u^{2} \mathbf{e}^{\prime}+\left(b+b^{\prime} u\right) u \mathbf{e}\right)=\mathbf{e}^{\prime}$.

This extension splits over an unramified extension if and only if $b=0$. If $F^{\prime} / \mathbf{Q}_{3}$ is non-abelian, then any descent data on $\mathcal{M}\left(2,1 ; 2,1 ;\left(b+b^{\prime} u\right) u\right)^{\prime}$ compatible with the standard descent data on $\mathcal{M}(2,1)^{\prime}$ satisfies

$$
\widehat{\gamma_{3}}(\mathbf{e})=H_{\gamma_{3}}(u)^{6} \mathbf{e}, \widehat{\gamma_{3}}\left(\mathbf{e}^{\prime}\right)=H_{\gamma_{3}}(u)^{6} \mathbf{e}^{\prime}+h_{\gamma_{3}}(u) \mathbf{e},
$$

where

$$
h_{\gamma_{3}}(0)=-b H_{\gamma_{3}}^{\prime}(0) .
$$

The sign in $h_{\gamma_{3}}(0)=-b H_{\gamma_{3}}^{\prime}(0)$ will be very important in 7.4 . The proof of this lemma is essentially the same as that of Lemma 7.2.5, but we repeat it anyway. 
Proof. The classification of extensions of Breuil modules follows from Lemma 5.2.2 The computation of which of these split over an unramified extension follows from Lemma 5.2.2 and Corollary 5.4.2.

Now suppose that $F^{\prime} / \mathbf{Q}_{3}$ is non-abelian. By Lemma 5.7.1 the only issue is to compute $h_{\gamma_{3}}(0)$. Since $H_{\gamma_{3}}(0) \equiv 1 \bmod 3$, by evaluating the congruence

$$
\widehat{\gamma_{3}} \circ \phi_{1}^{\prime} \equiv \phi_{1}^{\prime} \circ \widehat{\gamma_{3}} \bmod u \mathcal{M}\left(2,1 ; 2,1 ;\left(b+b^{\prime} u\right) u\right)^{\prime}
$$

on $u \mathbf{e}^{\prime}+\left(b+b^{\prime} u\right) \mathbf{e}$ and comparing constant terms of the coefficients of $\mathbf{e}$ on both sides we get

$$
\begin{aligned}
h_{\gamma_{3}}(0) & =h_{\gamma_{3}}(0)^{3}+\left.b\left(\frac{1-H_{\gamma_{3}}(u)}{u}\right)^{3}\right|_{u=0} \\
& =h_{\gamma_{3}}(0)^{3}-b H_{\gamma_{3}}^{\prime}(0)^{3}=h_{\gamma_{3}}(0)^{3}+b H_{\gamma_{3}}^{\prime}(0)
\end{aligned}
$$

in $\mathbf{F}_{9}$, where we have used the equality $H_{\gamma_{3}}^{\prime}(0)^{2}=-1$ (see $₫ 6.2$ ).

In other words $h_{\gamma}(0)$ is a root of $T^{3}-T+b H_{\gamma_{3}}^{\prime}(0)=0$. Since $H_{\gamma_{3}}^{\prime}(0)^{2}=-1$, we must have $h_{\gamma_{3}}(0)=-b H_{\gamma_{3}}^{\prime}(0)+a$ for some $a \in \mathbf{F}_{3}$. Since $\gamma_{2}\left(H_{\gamma_{3}}(u)\right)=H_{\gamma_{3}^{-1}}(u)$ and $\gamma_{2}\left(h_{\gamma_{3}}(u)\right)=h_{\gamma_{3}^{-1}}(u)$ are forced by the identity $\gamma_{2}(\pi)=\pi$, we see that $h_{\gamma_{3}^{-1}}(0)=$ $-b H_{\gamma_{3}^{-1}}^{\prime}(0)+a$ for the same $a \in \mathbf{F}_{3}$. The identity

$$
\widehat{\gamma_{3}} \circ \widehat{\gamma_{3}^{-1}} \circ \phi_{1}^{\prime} \equiv \phi_{1}^{\prime} \bmod u \mathcal{M}\left(2,1 ; 2,1 ; b+b^{\prime} u\right)^{\prime}
$$

then implies $h_{\gamma_{3}}(0)+h_{\gamma_{3}^{-1}}(0)=0$, so $a=0$.

\subsection{Rank three calculations.}

Lemma 7.3.1. Suppose that $\mathcal{G}$ is a finite flat group scheme over $\mathcal{O}_{F}$ which is killed by 3. Suppose that there is a filtration by closed finite flat subgroup schemes $\mathfrak{G}_{1} \subset$ $\mathcal{G}_{2} \subset \mathcal{G}$ such that $\mathcal{G}_{1} \cong \mathcal{G}(1, \delta), \mathcal{G}_{2} / \mathcal{G}_{1} \cong \mathcal{G}(2,1)$ and $\mathcal{G} / \mathcal{G}_{2} \cong \mathcal{G}(1, \delta)$. Suppose finally that $\mathcal{G}_{2} \times_{\mathcal{O}_{F}} F^{\prime}$ descends to $\mathbf{Q}_{3}$ in such a way that it is a très ramifié extension of $\mu_{3}$ by $\mathbf{Z} / 3 \mathbf{Z}$. Then

$$
\mathcal{G} / \mathcal{G}_{1} \cong \mathcal{G}(2,1) \oplus \mathcal{G}(1, \delta)
$$

compatibly with the extension class structure.

Proof. Let $\mathcal{M}=\mathcal{M}_{\pi}(\mathcal{G})$ and $\mathcal{N}=\mathcal{M}_{\pi}\left(\mathcal{G} / \mathcal{G}_{1}\right)$. Using Lemmas 7.2.1 and 7.2.3 we see that we can write

- $\mathcal{M}=\left(\mathbf{F}_{3}[u] / u^{9}\right) \mathbf{e}_{1} \oplus\left(\mathbf{F}_{3}[u] / u^{9}\right) \mathbf{e}_{\omega} \oplus\left(\mathbf{F}_{3}[u] / u^{9}\right) \mathbf{e}_{1}^{\prime}$,

- $\mathcal{M}_{1}=\left\langle u \mathbf{e}_{1}, u^{2} \mathbf{e}_{\omega}+b \mathbf{e}_{1}, u \mathbf{e}_{1}^{\prime}+\left(c+c^{\prime} u\right) \mathbf{e}_{\omega}+f \mathbf{e}_{1}\right\rangle$

for $b, c, c^{\prime} \in \mathbf{F}_{3}$ with $c \neq 0$ and with $f \in \mathbf{F}_{3}[u] / u^{9}$. It suffices to show $b=0$. Since we must have $u^{3} \mathcal{M} \subseteq \mathcal{M}_{1}$, we see that

$$
\begin{gathered}
\left(c+c^{\prime} u\right)\left(u^{2} \mathbf{e}_{\omega}+b \mathbf{e}_{1}\right)-u^{2}\left(u \mathbf{e}_{1}^{\prime}+\left(c+c^{\prime} u\right) \mathbf{e}_{\omega}+f \mathbf{e}_{1}\right)+u^{3} \mathbf{e}_{1}^{\prime} \\
=\left(b c+b c^{\prime} u-u^{2} f\right) \mathbf{e}_{1} \in \mathcal{M}_{1} .
\end{gathered}
$$

The Breuil module $\mathcal{N}$ is spanned as a $\mathbf{F}_{3}[u] / u^{9}$-module by $\mathbf{e}_{1}$ and $\mathbf{e}_{\omega}$, so by Lemma 7.2.1] $u$ must divide $b c+b c^{\prime} u-u^{2} f$. As $c \neq 0$ we must have $b=0$, as desired.

Combining this with Lemma 7.2.1 and the injectivity of $H^{1}\left(G_{3}, \omega\right) \rightarrow H^{1}\left(G_{F^{\prime}}, \omega\right)$ we get the following corollary, which is also the first part of Theorem 4.7.3, 
Corollary 7.3.2. The natural map

$$
\theta_{0}: \operatorname{Ext}_{\mathcal{S}_{ \pm 1}}^{1}(\bar{\rho}, \bar{\rho}) \longrightarrow H^{1}\left(G_{3}, \omega\right)
$$

is zero.

7.4. Conclusion of the proof of Theorem 4.4.1. Consider first the case of $F_{1}$. We still have to explain why

$$
\bar{\theta}_{\omega}: H_{\mathcal{S}_{1}}^{1}\left(G_{3}, \operatorname{ad}^{0} \bar{\rho}\right) \longrightarrow H^{1}\left(I_{3}, \mathbf{F}_{3}\right)
$$

is zero. Suppose $x \in H_{\mathcal{S}_{1}}^{1}\left(G_{3}, \operatorname{ad}^{0} \bar{\rho}\right)$ does not map to zero in $H^{1}\left(I_{3}, \mathbf{F}_{3}\right)$.

By our hypothesis on $x$ we may choose a totally ramified abelian cubic extension $\widetilde{F}_{1} / \mathbf{Q}_{3}$ such that $x$ restricts to zero under the natural map $H^{1}\left(G_{3}, \operatorname{ad}^{0} \bar{\rho}\right) \rightarrow$ $H^{1}\left(G_{\widetilde{F}_{1}}, \mathbf{F}_{3}\right)$. Then the image of $x$ under the natural map $H^{1}\left(G_{3}, \operatorname{ad}^{0} \bar{\rho}\right) \rightarrow$ $H^{1}\left(G_{\widetilde{F}_{1}}, \bar{\rho} \otimes \omega\right)$ is the image of some $\widetilde{x} \in H^{1}\left(G_{\widetilde{F}_{1}}, \omega\right)$ under the natural map $H^{1}\left(G_{\widetilde{F}_{1}}, \omega\right) \rightarrow H^{1}\left(G_{\widetilde{F}_{1}}, \bar{\rho} \otimes \omega\right)$. The element $\widetilde{x}$ parametrises a finite flat $\widetilde{F}_{1}$-group scheme $H$ which is an extension of $\mu_{3}$ by $\mathbf{Z} / 3 \mathbf{Z}$ and which is a subquotient of the restriction to $G_{\widetilde{F}_{1}}$ of the extension of $\bar{\rho}$ by itself parametrised by $x$. It follows that $H$ has a finite flat model $\mathcal{H} / \mathcal{O}_{\widetilde{F}}$ (see Lemma 4.1.1) and the special fibre of $\mathcal{H}$ must be local-local (if $\widetilde{x}=0$, then the extension of $\bar{\rho}$ by itself parametrised by $x$ splits over $\widetilde{F}_{1}$ and this is clear, while if $\widetilde{x} \neq 0$ we would otherwise get a contradiction from the connected-étale sequence). By Lemma 7.2.2, we may therefore lift $\widetilde{x}$ to $H^{1}\left(G_{3}, \omega\right)$. Using the commutative diagram

$$
\begin{array}{ccc}
H^{1}\left(G_{3}, \omega\right) & \longrightarrow & H^{1}\left(G_{3}, \bar{\rho} \otimes \omega\right) \\
\operatorname{res} \downarrow & & \downarrow \\
H^{1}\left(G_{\widetilde{F}_{1}}, \omega\right) & \longrightarrow & H^{1}\left(G_{\widetilde{F}_{1}}, \bar{\rho} \otimes \omega\right)
\end{array}
$$

and noting that the right-hand vertical map is injective we conclude that

$$
x \in H_{\mathcal{S}_{1}}^{1}\left(G_{3}, \operatorname{ad}^{0} \bar{\rho}\right) \subset H^{1}\left(G_{3}, \bar{\rho} \otimes \omega\right)
$$

is in the image of $H^{1}\left(G_{3}, \omega\right) \rightarrow H^{1}\left(G_{3}, \bar{\rho} \otimes \omega\right)$, a contradiction with the hypothesis that even the image of $x$ in $H^{1}\left(I_{3}, \mathbf{F}_{3}\right)$ is non-zero.

Now consider the case $F^{\prime}=F_{-1}^{\prime}$ which is non-abelian over $\mathbf{Q}_{3}$. We must show that

$$
\bar{\theta}_{\omega}: \operatorname{Ext}_{\mathcal{S}_{-1}}^{1}(\bar{\rho}, \bar{\rho}) \longrightarrow H^{1}\left(I_{3}, \mathbf{F}_{3}\right)
$$

is zero.

An element $x \in \operatorname{Ext}_{\mathcal{S}_{-1}}^{1}(\bar{\rho}, \bar{\rho})$ gives rise to a finite flat $\mathcal{O}_{F_{-1}}$-group scheme $\mathcal{G}$ killed by 3 and descent data $\{[g]\}$ for $F_{-1}^{\prime} / \mathbf{Q}_{3}$ on $\mathcal{G}^{\prime}=\mathcal{G} \times \times_{\mathcal{O}_{F_{-1}^{\prime}}} F_{-1}^{\prime}$, such that $\left(\mathcal{G}^{\prime},\{[g]\}\right)_{\mathbf{Q}_{3}}$ corresponds to the extension of $\bar{\rho}$ by itself classified by $x$. Let $\mathcal{N}$ denote the Breuil module for $\mathcal{G}$ and let $\mathcal{N}^{\prime}=\mathcal{N} \otimes \mathbf{F}_{9}$. According to Lemmas 7.2.1, 7.2.3, 7.2.5 77.2.6 and 7.3.1 we see that we can write

$$
\mathcal{N}=\left(\mathbf{F}_{3}[u] / u^{9}\right) \mathbf{e}_{\omega} \oplus\left(\mathbf{F}_{3}[u] / u^{9}\right) \mathbf{e}_{1} \oplus\left(\mathbf{F}_{3}[u] / u^{9}\right) \mathbf{e}_{\omega}^{\prime} \oplus\left(\mathbf{F}_{3}[u] / u^{9}\right) \mathbf{e}_{1}^{\prime}
$$

with

$$
\begin{aligned}
& \mathcal{N}_{1}=\left\langle u^{2} \mathbf{e}_{\omega}, u \mathbf{e}_{1}+\left(c+c^{\prime} u\right) \mathbf{e}_{\omega}, u^{2} \mathbf{e}_{\omega}^{\prime}+\left(a u+a^{\prime} u^{2}\right) \mathbf{e}_{\omega},\right. \\
&\left.u \mathbf{e}_{1}^{\prime}+\left(c+c^{\prime} u\right) \mathbf{e}_{\omega}^{\prime}+\left(b+b^{\prime} u\right) \mathbf{e}_{1}+h \mathbf{e}_{\omega}\right\rangle,
\end{aligned}
$$

where $h \in \mathbf{F}_{3}[u] / u^{9}$ is some polynomial and where $a, a^{\prime}, b, b^{\prime}, c, c^{\prime} \in \mathbf{F}_{3}$ with $c \neq 0$ (as $\bar{\rho}$ is très ramifié). By Lemma [7.2.6 what we must show is that $a=0$. 
Note that $H_{\gamma_{3}}^{\prime}(0) \neq 0$ in $\mathbf{F}_{9}$ by 86.2 By Lemmas 5.7 .1 and 7.2 .1 , the action $\widehat{\gamma_{3}}$ is determined by

$$
\begin{gathered}
\widehat{\gamma_{3}}\left(\mathbf{e}_{\omega}\right)=H_{\gamma_{3}}(u)^{6} \mathbf{e}_{\omega}, \widehat{\gamma_{3}}\left(\mathbf{e}_{1}\right)=H_{\gamma_{3}}(u)^{3} \mathbf{e}_{1}+g_{\gamma_{3}}(u) \mathbf{e}_{\omega}^{\prime}, \\
\widehat{\gamma_{3}}\left(\mathbf{e}_{\omega}^{\prime}\right)=H_{\gamma_{3}}(u)^{6} \mathbf{e}_{\omega}^{\prime}+h_{\gamma_{3}, \omega}(u) \mathbf{e}_{\omega}, \\
\widehat{\gamma_{3}}\left(\mathbf{e}_{\omega}^{\prime}\right)=H_{\gamma_{3}}(u)^{3} \mathbf{e}_{1}^{\prime}+g_{\gamma_{3}}(u) \mathbf{e}_{\omega}^{\prime}+h_{\gamma_{3}, \mathbf{1}} \mathbf{e}_{1}+G_{\gamma_{3}}(u) \mathbf{e}_{\omega},
\end{gathered}
$$

where $g_{\gamma_{3}}(u), G_{\gamma_{3}}(u) \in \mathbf{F}_{9}[u] / u^{9}$ and $h_{\gamma_{3}, \omega}$ and $h_{\gamma_{3}, \mathbf{1}}$ are as in Lemmas 7.2 .6 and 7.2.5 respectively.

Due to the requirement $\widehat{\gamma_{3}}\left(\mathcal{N}_{1}^{\prime}\right) \subseteq \mathcal{N}_{1}^{\prime}$, we must have

$$
\widehat{\gamma_{3}}\left(u \mathbf{e}_{1}^{\prime}+\left(c+c^{\prime} u\right) \mathbf{e}_{\omega}^{\prime}+\left(b+b^{\prime} u\right) \mathbf{e}_{1}+h(u) \mathbf{e}_{\omega}\right) \in \mathcal{N}_{1}^{\prime},
$$

and this element is obviously equal to

$$
\begin{array}{r}
\left(u H_{\gamma_{3}}\right)\left(H_{\gamma_{3}}^{3} \mathbf{e}_{1}^{\prime}+g_{\gamma_{3}} \mathbf{e}_{\omega}^{\prime}+h_{\gamma_{3}, \mathbf{1}} \mathbf{e}_{1}+G_{\gamma_{3}} \mathbf{e}_{\omega}\right)+\left(c+c^{\prime} u H_{\gamma_{3}}\right)\left(H_{\gamma_{3}}^{6} \mathbf{e}_{\omega}^{\prime}+h_{\gamma_{3}, \omega} \mathbf{e}_{\omega}\right) \\
+\left(b+b^{\prime} u H_{\gamma_{3}}\right)\left(H_{\gamma_{3}}^{3} \mathbf{e}_{1}+g_{\gamma_{3}} \mathbf{e}_{\omega}\right)+h\left(u H_{\gamma_{3}}\right) H_{\gamma_{3}}^{6} \mathbf{e}_{\omega} .
\end{array}
$$

We now try to express this as a linear combination of the generators of $\mathcal{N}_{1}^{\prime}$ listed in (7.4.1), while working modulo $\left\langle u^{3} \mathcal{N}^{\prime}, u^{2} \mathbf{e}_{\omega}\right\rangle \subseteq \mathcal{N}_{1}^{\prime}$. Using that $H_{\gamma_{3}}(0)=1$ in $\mathbf{F}_{9}$ and $h\left(u H_{\gamma_{3}}\right) \equiv h(u) \bmod u^{2}$, we arrive at the expression

$$
\begin{aligned}
H_{\gamma_{3}}\left(u \mathbf{e}_{1}^{\prime}+(c+\right. & \left.\left.c^{\prime} u\right) \mathbf{e}_{\omega}^{\prime}+\left(b+b^{\prime} u\right) \mathbf{e}_{1}+h \mathbf{e}_{\omega}\right) \\
& +\left(\frac{c\left(\left(1-H_{\gamma_{3}}\right) / u\right)+g_{\gamma_{3}}}{u}\right)\left(u^{2} \mathbf{e}_{\omega}^{\prime}+\left(a u+a^{\prime} u^{2}\right) \mathbf{e}_{\omega}\right) \\
& +\left(H_{\gamma_{3}} h_{\gamma_{3}, \mathbf{1}}+b\left(\frac{1-H_{\gamma_{3}}}{u}\right)\right)\left(u \mathbf{e}_{1}+\left(c+c^{\prime} u\right) \mathbf{e}_{\omega}\right)+F_{\gamma_{3}}(u) \mathbf{e}_{\omega}
\end{aligned}
$$

where

$$
\begin{aligned}
F_{\gamma_{3}}(u)= & u H_{\gamma_{3}} G_{\gamma_{3}}+\left(c+c^{\prime} u H_{\gamma_{3}}\right) h_{\gamma_{3}, \omega}+\left(b+b^{\prime} u H_{\gamma_{3}}\right) g_{\gamma_{3}}+h(u)\left(1-H_{\gamma_{3}}\right) \\
& -\left(a+a^{\prime} u\right)\left(c\left(1-H_{\gamma_{3}}\right) / u+g_{\gamma_{3}}\right)-\left(c+c^{\prime} u\right)\left(H_{\gamma_{3}} h_{\gamma_{3}, \mathbf{1}}+b\left(\left(1-H_{\gamma_{3}}\right) / u\right)\right)
\end{aligned}
$$

in $\mathbf{F}_{9}[u] / u^{9}$. In particular, $c\left(1-H_{\gamma_{3}}(u)\right) / u+g_{\gamma_{3}} \equiv 0 \bmod u$ and $F_{\gamma_{3}}(u) \equiv 0 \bmod u^{2}$. The condition $c\left(\left(1-H_{\gamma_{3}}\right) / u\right)+g_{\gamma_{3}} \equiv 0 \bmod u$ can be reformulated as

$$
g_{\gamma_{3}}(0)=c H_{\gamma_{3}}^{\prime}(0) \text {. }
$$

Since $F_{\gamma_{3}}(u) \equiv 0 \bmod u^{2}$, we have to have $F_{\gamma_{3}}(0)=0$. But a direct calculation using $g_{\gamma_{3}}(0)=\mathrm{cH}_{\gamma_{3}}^{\prime}(0)$ and the definition of $F_{\gamma_{3}}$ gives

$$
\begin{aligned}
F_{\gamma_{3}}(0) & =0+c h_{\gamma_{3}, \omega}(0)+b g_{\gamma_{3}}(0)+0-0-c\left(h_{\gamma_{3}, \mathbf{1}}(0)-b H_{\gamma_{3}}^{\prime}(0)\right) \\
& =c\left(h_{\gamma_{3}, \omega}(0)-h_{\gamma_{3}, \mathbf{1}}(0)-b H_{\gamma_{3}}^{\prime}(0)\right),
\end{aligned}
$$

so the non-vanishing of $c$ forces

$$
h_{\gamma_{3}, \omega}(0)-h_{\gamma_{3}, 1}(0)=b H_{\gamma_{3}}^{\prime}(0) .
$$

Lemmas 7.2.6 and 7.2.5 give us the values

$$
h_{\gamma_{3}, \omega}(0)=-a H_{\gamma_{3}}^{\prime}(0), \quad h_{\gamma_{3}, \mathbf{1}}(0)=-b H_{\gamma_{3}}^{\prime}(0) .
$$

Thus $(-a+b) H_{\gamma_{3}}^{\prime}(0)=b H_{\gamma_{3}}^{\prime}(0)$, and so $a=0$. This completes the proof of Theorem 4.7 .3 and hence of Theorem 4.4.1. 


\section{Proof of Theorem 4.5.1}

In this section we will keep the notation of $\$ 4.5$ and either $\$ 6.3$ or $\$ 6.4$ (depending on whether we are working with $\mathcal{S}_{3}$ or $\mathcal{S}_{-3}$ ). We will set $\delta= \pm 1$ in the case of $\mathcal{S}_{\mp 3}$ (so that $c_{\pi} \equiv \delta \bmod (3, u)$ ). Note the signs. We will write $F$ for $F_{ \pm 3}, F^{\prime}$ for $F_{ \pm 3}^{\prime}$ and $\mathcal{J}$ for $\mathcal{J}_{ \pm 3}$. If $\mathcal{G}$ (resp. $\mathcal{M}$ ) is a finite flat $\mathcal{O}_{F}$-group scheme (resp. Breuil module over $\mathcal{O}_{F}$ ) we will write $\mathcal{G}^{\prime}$ (resp. $\mathcal{M}^{\prime}$ ) for the base change to $\mathcal{O}_{F^{\prime}}$.

8.1. Rank one calculations. We remark that with our choice of polynomials $H_{g}(u)$ in $\S 6.3$ and $\S 6.4$, any object $\mathcal{M}$ in $\underline{\phi 1}_{1} D D_{F^{\prime} / \mathbf{Q}_{3}}$ has an action of $\left\langle\gamma_{2}, \gamma_{4}\right\rangle$ (via $\widehat{\gamma}_{2}$ and $\widehat{\gamma}_{4}$, the action of $\gamma_{2}$ being Frob ${ }_{3}$-semi-linear). Also, since $\gamma_{3}$ and $\gamma_{2}$ commute,

$H_{\gamma_{2}}=1$ and $H_{\gamma_{3}^{ \pm 1}}(u) \in \mathbf{Z}_{3}[u]$, we see that $\widehat{\gamma}_{2}$ must commute with $\widehat{\gamma_{3}^{ \pm 1}}$ by Corollary 5.6 .2

We recall from Lemma 5.2 .1 that the only models for $(\mathbf{Z} / 3 \mathbf{Z})_{/ F}$ over $\mathcal{O}_{F}$ are $\mathcal{G}(r, \delta)$ for $r=0,2,4,6,8,10,12$ with $\mathcal{G}(12, \delta) \cong(\mathbf{Z} / 3 \mathbf{Z})_{/ \mathcal{O}_{F}}$, and the only models for $\left(\mu_{3}\right)_{/ F}$ over $\mathcal{O}_{F}$ are $\mathcal{G}(r, 1)$ for $r=0,2,4,6,8,10,12$ with $\mathcal{G}(0,1) \cong\left(\mu_{3}\right)_{/ \mathcal{O}_{F}}$. In each case, the base change to $\mathcal{O}_{F^{\prime}}$ admits unique descent data over $\mathbf{Q}_{3}$ such that descent of the generic fibre to $\mathbf{Q}_{3}$ is $\mathbf{Z} / 3 \mathbf{Z}$ (resp. $\mu_{3}$ ). (See Lemma 5.7.1.) We will write $\mathcal{G}_{r, 1}^{\prime}$ (resp. $\left.\mathcal{G}_{r, \omega}^{\prime}\right)$ for the corresponding pair $\left(\mathcal{G}(r, \delta) \times \mathcal{O}_{F^{\prime}},\{[g]\}\right)$ (resp. $\left.\left(\mathcal{G}(r, 1) \times \mathcal{O}_{F^{\prime}},\{[g]\}\right)\right)$. We will also let $\mathcal{M}_{r, 1}^{\prime}$ (resp. $\left.\mathcal{M}_{r, \omega}^{\prime}\right)$ denote the corresponding object of $\underline{\phi 1}_{1} D D_{F^{\prime} / \mathbf{Q}_{3}}$. In particular, for $\chi=1$ or $\omega$, the underlying $\mathbf{F}_{9}[u] / u^{36}$ module has the form $\left(\mathbf{F}_{9}[u] / u^{36}\right) \mathbf{e}_{\chi}$ with $\mathbf{e}_{\chi}$ the standard generator, though we write $\mathbf{e}$ rather than $\mathbf{e}_{\chi}$ if $\chi$ is understood.

We have the following useful lemma.

Lemma 8.1.1. Let $0 \leq r \leq e=12$ be an even integer. The descent data on $\mathcal{M}_{r, 1}^{\prime}$ is determined by

$$
\widehat{\gamma_{2}}(\mathbf{e})=\mathbf{e}, \quad \widehat{\gamma_{4}}(\mathbf{e})=-(-\sqrt{-1})^{r / 2} \mathbf{e}, \widehat{\gamma_{3}^{ \pm 1}}(\mathbf{e})=H_{\gamma_{3}^{ \pm 1}}(u)^{-3 r / 2} \mathbf{e},
$$

and the descent data on $\mathcal{M}_{r, \omega}^{\prime}$ is determined by

$$
\widehat{\gamma_{2}}(\mathbf{e})=\mathbf{e}, \widehat{\gamma_{4}}(\mathbf{e})=(-\sqrt{-1})^{r / 2} \mathbf{e}, \widehat{\gamma_{3}^{ \pm 1}}(\mathbf{e})=H_{\gamma_{3}^{ \pm 1}}(u)^{-3 r / 2} \mathbf{e} .
$$

In particular, $\gamma_{4}^{2}=-1$ on $D\left(\mathcal{G}_{r, 1}^{\prime}\right)$ if and only if $\gamma_{4}^{2}=-1$ on $D\left(\mathcal{G}_{r, \omega}^{\prime}\right)$ if and only if $r=2,6$ or 10 .

Proof. Certainly $\widehat{\gamma_{2}}(\mathbf{e})=\mathbf{e}$. We have already seen in Lemma 5.7.1 that descent data must exist in each case, so our task is to compute the unique units $\xi_{\gamma_{4}}, \xi_{\gamma_{3} \pm 1} \in$ $\left(\mathbf{F}_{9}[u] / u^{36}\right)^{\times}$so that

$$
\widehat{\gamma_{4}}(\mathbf{e})=\xi_{\gamma_{4}} \mathbf{e}, \widehat{\gamma_{3}^{ \pm 1}}(\mathbf{e})=\xi_{\gamma_{3} \pm 1} \mathbf{e}
$$

corresponds to generic fibre descent data for the mod 3 cyclotomic or trivial character on $G_{3}$. The case of $\gamma_{3}{ }^{ \pm 1}$ follows from Lemma 5.7.1

From the condition

$$
\widehat{\gamma_{4}} \circ \phi_{1}^{\prime}\left(u^{r} \mathbf{e}\right)=\phi_{1}^{\prime} \circ \widehat{\gamma_{4}}\left(u^{r} \mathbf{e}\right)
$$

we get $\xi_{\gamma_{4}}^{2}(u)=(-\sqrt{-1})^{r}$, so

$$
\xi_{\gamma_{4}}(u)= \pm(-\sqrt{-1})^{r / 2} .
$$

The non-zero morphisms $\mathcal{M}_{r, 1} \rightarrow \mathcal{M}_{12,1}$ are given by $\mathbf{e} \mapsto \pm u^{3(12-r) / 2} \mathbf{e}$ and the non-zero morphisms $\mathcal{M}_{0, \omega} \rightarrow \mathcal{M}_{r, \omega}$ are given by $\mathbf{e} \mapsto \pm u^{3 r / 2} \mathbf{e}$. Thus, it suffices to 
check that $\widehat{\gamma_{4}} \mathbf{e}=\mathbf{e}$ on $\mathcal{M}_{12,1}^{\prime}$ and $\widehat{\gamma_{4}} \mathbf{e}=\mathbf{e}$ on $\mathcal{M}_{0, \omega}^{\prime}$. In both cases we have shown that $\widehat{\gamma_{4}} \mathbf{e}= \pm \mathbf{e}$ and so we only need to check that $\gamma_{4}=1$ on $D\left(\mathcal{G}_{12,1}^{\prime}\right)$ and $D\left(\mathcal{G}_{0, \omega}^{\prime}\right)$. That is, we have to show that the $\mathcal{O}_{F^{\prime}}$-group scheme maps $\mathbf{Z} / 3 \mathbf{Z} \rightarrow \gamma_{4}(\mathbf{Z} / 3 \mathbf{Z})$ and $\mu_{3} \rightarrow{ }^{\gamma_{4}} \mu_{3}$ arising from the canonical generic fibre descent data induce the identity on the special fibres. This is easy.

Lemma 8.1.2. Let $\mathcal{M}$ be an object of $\phi_{1}-\bmod _{F}$ corresponding to a finite flat group scheme $\mathcal{G}$ and let $\{[g]\}$ be descent data on $\mathcal{G}^{\prime}=\mathcal{G} \times \mathcal{O}_{F^{\prime}}$ relative to $\mathbf{Q}_{3}$. Assume that $\left(\mathcal{G}^{\prime},\{\widehat{g}\}\right)_{\mathbf{Q}_{3}}$ can be filtered so that each graded piece is isomorphic to $\mathbf{Z} / 3 \mathbf{Z}$ or $\mu_{3}$ and so that the corresponding filtration of $\left(\mathcal{M}^{\prime},\{\widehat{g}\}\right)$ in ${\underline{\phi_{1} D D}}_{F^{\prime} / \mathbf{Q}_{3}}$ has successive quotients of the form $\mathcal{M}_{r_{j}, \chi_{j}}^{\prime}$ with $r_{j} \in\{2,6,10\}$ and $\chi_{j} \in\{\mathbf{1}, \omega\}$. Then $\gamma_{4}^{2}=-1$ on $\mathcal{M}^{\prime} / u \mathcal{M}^{\prime}$ and there exists a basis $\left\{\mathbf{e}_{j}\right\}$ of $\mathcal{M}$ over $\mathbf{F}_{3}[u] / u^{36}$ so that for all $j$

- $\mathbf{e}_{j} \in \phi_{1}\left(\mathcal{M}_{1}\right)$,

- $\mathbf{e}_{j}$ is an eigenvector of the $\mathbf{F}_{9}$-linear map $\widehat{\gamma_{4}}$ on $\mathcal{M}^{\prime}$,

- $\mathbf{e}_{j}$ lies in the part of the filtration of $\mathcal{M}^{\prime}$ which surjects onto $\mathcal{M}_{r_{j}, \chi_{j}}^{\prime}$ and this surjection sends $\mathbf{e}_{j}$ onto the standard basis vector $\mathbf{e}$ of $\mathcal{M}_{r_{j}, \chi_{j}}^{\prime}$ over $\mathbf{F}_{9}[u] / u^{36}$.

Proof. Since $\gamma_{4}^{2}$ acts linearly on $\mathcal{M}^{\prime} / u \mathcal{M}^{\prime}$ and $\left(\gamma_{4}^{2}\right)^{2}=1$, the action of $\gamma_{4}^{2}$ must be semi-simple. The eigenvalues of $\gamma_{4}^{2}$ are all equal to -1 , so necessarily $\gamma_{4}^{2}=-1$ on $\mathcal{M}^{\prime} / u \mathcal{M}^{\prime}$.

We now argue by induction on the number of Jordan-Hölder factors in the generic fibre, the case of length 1 being clear. Thus, we can assume we have a short exact sequence in $\underline{\phi}_{1} D D_{F^{\prime} / \mathbf{Q}_{3}}$,

$$
0 \longrightarrow \mathcal{N}^{\prime} \longrightarrow \mathcal{M}^{\prime} \longrightarrow \mathcal{M}_{r, \chi}^{\prime} \longrightarrow 0
$$

so the lemma is known for $\mathcal{N}^{\prime}$. We just have to find $\mathbf{e}_{0} \in \phi_{1}\left(\mathcal{M}_{1}\right)$ mapping onto the standard basis vector $\mathbf{e}$ in $\mathcal{M}_{r, \chi}^{\prime}$ such that $\mathbf{e}_{0}$ is an eigenvector of $\widehat{\gamma}_{4}$. Since $\phi_{1}^{\prime}\left(\mathcal{M}_{1}^{\prime}\right) \rightarrow \phi_{1}^{\prime}\left(\left(\mathcal{M}_{r, \chi}^{\prime}\right)_{1}\right)$ is a surjective map of $\mathbf{F}_{9}$-vector spaces which is compatible with the semi-simple $\mathbf{F}_{9}$-linear endomorphism $\widehat{\gamma}_{4}$ on each side, we can find $\mathbf{e}_{0}^{\prime} \in$ $\phi_{1}^{\prime}\left(\mathcal{M}_{1}^{\prime}\right)$ mapping onto e with $\mathbf{e}_{0}^{\prime}$ an eigenvector of $\widehat{\gamma}_{4}$, say $\widehat{\gamma}_{4}\left(\mathbf{e}_{0}^{\prime}\right)=(\sqrt{-1})^{ \pm 1} \mathbf{e}_{0}^{\prime}$. Since

$$
\widehat{\gamma}_{4} \circ \widehat{\gamma}_{2}\left(\mathbf{e}_{0}^{\prime}\right)=\widehat{\gamma}_{2} \circ \widehat{\gamma}_{4}^{3}\left(\mathbf{e}_{0}^{\prime}\right)=\widehat{\gamma}_{2}\left(\sqrt{-1}^{\mp 1} \mathbf{e}_{0}^{\prime}\right)=\sqrt{-1}^{ \pm 1} \widehat{\gamma}_{2}\left(\mathbf{e}_{0}^{\prime}\right),
$$

the element $\mathbf{e}_{0}=(1 / 2)\left(\mathbf{e}_{0}^{\prime}+\widehat{\gamma}_{2}\left(\mathbf{e}_{0}^{\prime}\right)\right)$ maps to $\mathbf{e}$ and is an an eigenvector for $\widehat{\gamma}_{4}$. Also, $\mathbf{e}_{0} \in \phi_{1}^{\prime}\left(\mathcal{M}_{1}^{\prime}\right)$ is $\widehat{\gamma}_{2}$-invariant and $\widehat{\gamma}_{2}$ commutes with $\phi_{1}^{\prime}$, so $\mathbf{e}_{0} \in \phi_{1}\left(\mathcal{M}_{1}\right)$.

\subsection{Models for $\bar{\rho}$.}

Proposition 8.2.1. There exists a unique object $\left(\mathcal{G}^{\prime},\{[g]\}\right)$ of $\mathcal{F D}_{F^{\prime} / \mathbf{Q}_{3}, \mathcal{J}}$ such that $\left(\mathcal{G}^{\prime},\{[g]\}\right)_{\mathbf{Q}_{3}}$ corresponds to $\bar{\rho}$. If we set $\left(\mathcal{M}(\bar{\rho})^{\prime},\{\widehat{g}\}\right)=\mathcal{M}_{\pi}\left(\mathcal{G}^{\prime},\{[g]\}\right)$, then $\left(\mathcal{M}(\bar{\rho})^{\prime},\{\widehat{g}\}\right)$ is an extension of $\mathcal{M}_{2, \omega}^{\prime}$ by $\mathcal{M}_{10,1}^{\prime}$ in $\underline{\phi}_{1} D D_{F^{\prime} / \mathbf{Q}_{3}}$. Moreover Frobenius is not identically zero on $D\left(\mathcal{G}^{\prime}\right)$.

Proof. Let $\left(\mathcal{G}^{\prime},\{[g]\}\right)$ be an object of $\mathcal{F D}_{F^{\prime} / \mathbf{Q}_{3}, \mathcal{J}}$ such that $\left(\mathcal{G}^{\prime},\{[g]\}\right)_{\mathbf{Q}_{3}}$ corresponds to $\bar{\rho}$, and set $\left(\mathcal{M}^{\prime},\{\widehat{g}\}\right)=\mathcal{M}_{\pi}\left(\mathcal{G}^{\prime},\{[g]\}\right)$. As in the discussion following Theorem 5.6.1, we have canonically $\mathcal{M}^{\prime} \simeq \mathbf{F}_{9} \otimes_{\mathbf{F}_{3}} \mathcal{M}$ for a Breuil module $\mathcal{M}$ over $\mathcal{O}_{F}$, with $\widehat{\gamma}_{2}$ acting as $\gamma_{2} \otimes 1$. By Lemma 8.1.1, there is a short exact sequence of Breuil modules over $\mathcal{O}_{F}$,

$$
0 \longrightarrow \mathcal{M}(s, \delta) \longrightarrow \mathcal{M} \longrightarrow \mathcal{M}(r, 1) \longrightarrow 0
$$


with $r, s \in\{2,6,10\}$ and this is compatible with descent data after base change to $\mathcal{O}_{F^{\prime}}$ in the sense that we obtain an exact sequence

$$
0 \longrightarrow \mathcal{M}_{s, 1}^{\prime} \longrightarrow \mathcal{M}^{\prime} \longrightarrow \mathcal{M}_{r, \omega}^{\prime} \longrightarrow 0,
$$

compatible with descent data. Because $\bar{\rho}$ is très ramifié, it follows that $\left.\bar{\rho}\right|_{G_{F^{\prime}}}$ is non-split, so the sequence

$$
0 \longrightarrow \mathcal{N}(s, \delta) \longrightarrow \mathcal{M} \longrightarrow \mathcal{M}(r, 1) \longrightarrow 0
$$

is non-split.

We first show that we must have $(r, s)=(2,10)$. Since $\bar{\rho}$ is self-dual, in order to prove $(r, s)=(2,10)$ we may use Cartier duality (and Lemma 5.2.1) in order to reduce to the case where $r+s \leq e=12$. We will first rule out cases with $r \geq s$ and then the case $(r, s)=(2,6)$.

By Lemmas 8.1.1 and 8.1.2, we can write

$$
\mathcal{M}=\left(\mathbf{F}_{3}[u] / u^{36}\right) \mathbf{e}_{1} \oplus\left(\mathbf{F}_{3}[u] / u^{36}\right) \mathbf{e}_{\omega}^{\prime}, \quad \mathcal{M}_{1}=\left\langle u^{s} \mathbf{e}_{1}, u^{r} \mathbf{e}_{\omega}^{\prime}+h \mathbf{e}_{1}\right\rangle
$$

for some $h \in \mathbf{F}_{3}[u] / u^{36}$ so that

$$
\phi_{1}\left(u^{s} \mathbf{e}_{1}\right)=\delta \mathbf{e}_{1}, \quad \phi_{1}\left(u^{r} \mathbf{e}_{\omega}^{\prime}+h \mathbf{e}_{1}\right)=\mathbf{e}_{\omega}^{\prime}
$$

and

$$
\widehat{\gamma}_{4}\left(\mathbf{e}_{1}\right)=-(-\sqrt{-1})^{s / 2} \mathbf{e}_{1}, \quad \widehat{\gamma}_{4}\left(\mathbf{e}_{\omega}^{\prime}\right)=(-\sqrt{-1})^{r / 2} \mathbf{e}_{\omega}^{\prime} .
$$

Recall from Lemma 5.2.2 that the "parameter" $h$ gives an isomorphism of abstract groups

$$
\left(\mathbf{F}_{3}[u] / u^{36}\right) /\left\{u^{s} t-\delta u^{r} t^{3} \mid t \in \mathbf{F}_{3}[u] / u^{36}\right\} \simeq \operatorname{Ext}_{\underline{\phi_{1}-\bmod _{F}}}^{1}(\mathcal{M}(r, 1), \mathcal{M}(s, \delta)) .
$$

It is easy to see that

$$
\widehat{\gamma}_{4}\left(\mathcal{M}_{1}^{\prime}\right) \subseteq \mathcal{M}_{1}^{\prime}, \quad \widehat{\gamma}_{4} \circ \phi_{1}^{\prime}=\phi_{1}^{\prime} \circ \widehat{\gamma}_{4} \text { on } \mathcal{M}_{1}^{\prime}
$$

if and only if $\widehat{\gamma}_{4}\left(u^{r} \mathbf{e}_{\omega}^{\prime}+h \mathbf{e}_{1}\right) \in \mathcal{M}_{1}^{\prime}$ and $\widehat{\gamma}_{4}\left(\mathbf{e}_{\omega}^{\prime}\right)=\phi_{1}^{\prime} \circ \widehat{\gamma}_{4}\left(u^{r} \mathbf{e}_{\omega}^{\prime}+h \mathbf{e}_{1}\right)$, or equivalently

$$
(\sqrt{-1})^{r / 2} h(u) \equiv-(-\sqrt{-1})^{s / 2} h(-\sqrt{-1} u) \bmod u^{12+s} .
$$

This says exactly that

$$
j \equiv 2-(r+s) / 2 \bmod 4
$$

for any $j<12+s$ with a non-zero $u^{j}$ term appearing in $h$.

If $(r, s)=(6,2)$ this would force $h \equiv 0 \bmod u^{2}$, yet $\left\{u^{2} t-\delta u^{6} t^{3} \mid t \in \mathbf{F}_{3}[u] / u^{36}\right\}$ contains all multiples of $u^{2}$, so

$$
0 \longrightarrow \mathcal{N}(2, \delta) \longrightarrow \mathcal{M} \longrightarrow \mathcal{M}(6,1) \longrightarrow 0
$$

is split, a contradiction.

When $(r, s)=(10,2)$ or $(r, s)=(2,2)$ we see that $h \equiv h(0) \bmod u^{4}$, yet

$$
u^{4}\left(\mathbf{F}_{3}[u] / u^{36}\right) \subseteq\left\{u^{s} t-\delta u^{r} t^{3} \mid t \in \mathbf{F}_{3}[u] / u^{36}\right\},
$$

so the choice of $\mathbf{e}_{\omega}^{\prime}$ may be changed in order to arrange that

$$
h \in \mathbf{F}_{3}
$$

(though making this change of basis of $\mathcal{M}$ may destroy the "diagonal" form of $\widehat{\gamma}_{4}$ ). Since

$$
0 \longrightarrow \mathcal{M}(s, \delta) \longrightarrow \mathcal{N} \longrightarrow \mathcal{N}(r, 1) \longrightarrow 0
$$


is non-split, necessarily $h \neq 0$, so by rescaling $\mathbf{e}_{\omega}^{\prime}$ it can be assumed that $h=1$. Then $\mathbf{V}_{\mathcal{M}}\left(\mathbf{e}_{\omega}^{\prime}\right) \equiv \mathbf{e}_{1} \bmod u \mathcal{M}$ (by Theorem 5.1 .3 ) and

$$
\phi\left(\mathbf{e}_{\omega}^{\prime}\right) \equiv-\left(\delta / c_{\pi}\right) u^{3(12-r-s)} \mathbf{e}_{1} \equiv-u^{3(12-r-s)} \mathbf{e}_{1} \bmod u \mathcal{M} .
$$

This forces $r+s=12$. In particular, $(r, s)=(2,2)$ is ruled out.

For $(r, s)=(10,2)$, a splitting of the generic fibre $\left.\bar{\rho}\right|_{F}$ is induced by the Breuil module map

$$
\mathcal{M}(0,1) \longrightarrow \mathcal{M}
$$

defined by

$$
\mathbf{e} \longmapsto u^{15} \mathbf{e}_{\omega}^{\prime}+u^{3} f \mathbf{e}_{1}=u^{5}\left(u^{10} \mathbf{e}_{\omega}^{\prime}+\mathbf{e}_{1}\right)+\left(u f-u^{3}\right) u^{2} \mathbf{e}_{1},
$$

where $f \in \mathbf{F}_{3}[u] / u^{36}$ satisfies $f^{3}-\delta f=u^{6}$ (i.e. $f=-\delta u^{6}-u^{18}$, and a constant $c \in \mathbf{F}_{3}$ can even be added to this if $\delta=1$ ). But $\left.\bar{\rho}\right|_{G_{F^{\prime}}}$ must be non-split, so this rules out $(r, s)=(10,2)$.

The remaining case with $r \geq s$ is $(r, s)=(6,6)$. In this case $\left\{u^{s} t-\delta u^{r} t^{3} \mid t \in\right.$ $\left.\mathbf{F}_{3}[u] / u^{36}\right\}$ contains all multiples of $u^{8}$. But we have $j \equiv 0 \bmod 4$ for all $j<12+s=$ 18 such that a non-zero $u^{j}$ term appears in $h$, so again (at the expense of possibly making the $\widehat{\gamma}_{4}$-action non-diagonal) we may assume

$$
h=c+c^{\prime} u^{4}
$$

for some $c, c^{\prime} \in \mathbf{F}_{3}$. Writing $\widehat{\gamma}_{4}\left(\mathbf{e}_{\omega}^{\prime}\right)=(\sqrt{-1}) \mathbf{e}_{\omega}^{\prime}+h_{\gamma_{4}}(u) \mathbf{e}_{1}$, the commutativity of $\widehat{\gamma}_{4}$ and $\phi_{1}^{\prime}$ amounts to $h_{\gamma_{4}}=-\delta h_{\gamma_{4}}^{3}$, so $h_{\gamma_{4}}(u)=b \sqrt{-\delta}$ for some $b \in \mathbf{F}_{3}$. The condition $\widehat{\gamma}_{4}^{4}\left(\mathbf{e}_{\omega}^{\prime}\right)=\mathbf{e}_{\omega}^{\prime}$ forces $b=0$, so $\widehat{\gamma}_{4}$ still has diagonal action. This analysis shows that the map of $\mathbf{F}_{3}$-vector spaces

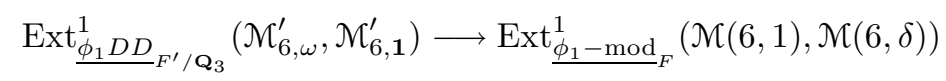

has at most a 2-dimensional image. If $c^{\prime}+\delta c=0$, then the Breuil module map

$$
\mathbf{F}_{9} \otimes \mathcal{M}(0,1) \longrightarrow \mathcal{M}^{\prime}
$$

defined by

$$
\mathbf{e} \longmapsto c \delta u^{7} \mathbf{e}_{1}+u^{3}\left(u^{6} \mathbf{e}_{\omega}^{\prime}+\left(c+c^{\prime} u^{4}\right) \mathbf{e}_{1}\right)
$$

gives a splitting of the corresponding representation of $G_{F^{\prime}}$. Thus the image of

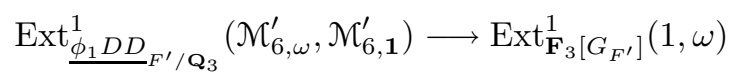

is at most one dimensional and, because $\left.\bar{\rho}\right|_{G_{F^{\prime}}}$ is non-split, the pair $\left(c, c^{\prime}\right)$ corresponding to a model of $\bar{\rho}$ satisfies $c^{\prime}+\delta c \neq 0$.

At this point, we treat the cases $\delta= \pm 1$ separately. Consider first the case $\delta=1$. We must have

$$
\widehat{\gamma}_{3}\left(\mathbf{e}_{1}\right)=H_{\gamma_{3}}(u)^{-9} \mathbf{e}_{1}, \quad \widehat{\gamma}_{3}\left(\mathbf{e}_{\omega}^{\prime}\right)=H_{\gamma_{3}}(u)^{-9} \mathbf{e}_{\omega}^{\prime}+h_{\gamma_{3}}(u) \mathbf{e}_{1},
$$

where $h_{\gamma_{3}}(u) \in \mathbf{F}_{9}[u] / u^{36}$ lies in $\mathbf{F}_{3}[u] / u^{36}$ because $\widehat{\gamma_{3}}$ commutes with $\widehat{\gamma}_{2}$. Evaluating $\widehat{\gamma}_{3} \circ \phi_{1}^{\prime} \equiv \phi_{1}^{\prime} \circ \widehat{\gamma}_{3} \bmod u \mathcal{M}^{\prime}$ on $u^{6} \mathbf{e}_{\omega}^{\prime}+\left(c+c^{\prime} u^{4}\right) \mathbf{e}_{1} \in \mathcal{M}_{1}^{\prime}$ and using our knowledge of $H_{\gamma_{3}}(u) \bmod 3$, we arrive at

$$
h_{\gamma_{3}}(0)=\delta\left(h_{\gamma_{3}}(0)^{3}+\left(c+c^{\prime}\right)\right)
$$

which is impossible for $h_{\gamma_{3}}(0) \in \mathbf{F}_{3}$ with $\delta=1$ because $c+c^{\prime}=c+\delta c^{\prime} \in \mathbf{F}_{3}^{\times}$. 
Now let us turn to the case $\delta=-1$, still in the case $(r, s)=(6,6)$. In this case $\operatorname{Ext}_{\mathbf{F}_{3}\left[G_{3}\right]}^{1}(1, \omega) \rightarrow \operatorname{Ext}_{\mathbf{F}_{3}\left[G_{F^{\prime}}\right]}^{1}(1, \omega)$ is injective and so by 8.2 .2$)$ we see that the image of

$$
\operatorname{Ext}_{\underline{\phi_{1} D D}}^{1}{ }_{F^{\prime} / \mathbf{Q}_{3}}\left(\mathcal{M}_{6, \omega}^{\prime}, \mathcal{M}_{6, \mathbf{1}}^{\prime}\right) \longrightarrow \operatorname{Ext}_{\mathbf{F}_{3}\left[G_{3}\right]}^{1}(1, \omega)
$$

is at most one dimensional. Thus to exclude the case $(r, s)=(6,6)$ and $\delta=-1$, it suffices to show that this image contains the peu ramifié line (as $\bar{\rho}$ is très ramifié). By Proposition 5.2.1 of [Man], there is an elliptic curve $E_{/ \mathbf{Q}_{3}}^{\prime}$ which has supersingular reduction over $\mathbf{Q}_{3}(\sqrt{-1}, \beta)$, with $\bar{\rho}_{E^{\prime}, 3}$ a non-split, peu ramifié extension of 1 by $\omega$. The representation $\left.\bar{\rho}_{E^{\prime}, 3}\right|_{F^{\prime}}$ is non-split (again because $H^{1}\left(G_{3}, \omega\right) \rightarrow H^{1}\left(G_{F^{\prime}}, \omega\right)$ is injective in the $\delta=-1$ case). Let $\mathcal{N}^{\prime}$ be the Breuil module corresponding to the 3 -torsion on the Néron model of $E^{\prime} \times \mathbf{Q}_{3} F^{\prime}$, so $\mathcal{N}^{\prime}$ admits descent data $\left\{\widehat{g}^{\prime}\right\}$ via the universal property of Néron models. The filtration of $\bar{\rho}$ induces a short exact sequence in $\underline{\phi 1}_{1} D F_{F^{\prime}} / \mathbf{Q}_{3}$

$$
0 \longrightarrow \mathcal{M}_{a, \mathbf{1}}^{\prime} \longrightarrow\left(\mathcal{N}^{\prime},\left\{\widehat{g}^{\prime}\right\}\right) \longrightarrow \mathcal{M}_{b, \omega}^{\prime} \longrightarrow 0
$$

for some even $a, b$ with $2 \leq a, b \leq 10$. The Néron model of $E^{\prime} \times \mathbf{Q}_{3} \mathbf{Q}_{3}(\sqrt{-1}, \beta)$ has local-local 3-torsion, and the induced local-local integral models $\mathcal{G}_{\omega}$ and $\mathcal{G}_{1}$ of the diagonal characters $\left.\omega\right|_{\mathbf{Q}_{3}(\sqrt{-1}, \beta)}$ and $\left.1\right|_{\mathbf{Q}_{3}(\sqrt{-1}, \beta)}$ must be the unique local-local models (uniqueness follows from Corollary 1.5.1 of [Ra]). Moreover, Corollary 1.5.1 of [Ra makes it clear that base change to $\mathcal{O}_{F^{\prime}}$ takes the order 3 group schemes $\mathcal{S}_{\omega}$ and $\mathcal{G}_{1}$ to the integral models that lie in the middle of the well-ordered sets of integral models of $\left.\omega\right|_{F^{\prime}}$ and $\left.1\right|_{F^{\prime}}$. It follows that $a=b=6$, so the map

$$
\operatorname{Ext}_{\underline{\phi_{1} D D}}^{1}{\underline{F^{\prime} / \mathbf{Q}_{3}}}\left(\mathcal{M}_{6, \omega}^{\prime}, \mathcal{M}_{6, \mathbf{1}}^{\prime}\right) \longrightarrow \operatorname{Ext}_{\mathbf{F}_{3}\left[G_{3}\right]}^{1}(1, \omega)
$$

indeed hits the peu ramifié line.

We next exclude the case $(r, s)=(2,6)$. As a first step, we check that there is at most one possibility for the underlying Breuil module $\mathcal{M}$ (ignoring the extension class structure) if $(r, s)=(2,6)$. We can write

$$
\mathcal{M}=\left(\mathbf{F}_{3}[u] / u^{36}\right) \mathbf{e}_{1} \oplus\left(\mathbf{F}_{3}[u] / u^{36}\right) \mathbf{e}_{\omega}^{\prime}, \quad \mathcal{M}_{1}=\left\langle u^{6} \mathbf{e}_{1}, u^{2} \mathbf{e}_{\omega}^{\prime}+h \mathbf{e}_{1}\right\rangle
$$

for some necessarily non-zero $h \in \mathbf{F}_{3}[u] / u^{36}$ with

$$
\phi_{1}\left(u^{6} \mathbf{e}_{1}\right)=\delta \mathbf{e}_{1}, \quad \phi_{1}\left(u^{2} \mathbf{e}_{\omega}^{\prime}+h \mathbf{e}_{1}\right)=\mathbf{e}_{\omega}^{\prime}
$$

and

$$
\widehat{\gamma}_{4}\left(\mathbf{e}_{1}\right)=-\sqrt{-1} \mathbf{e}_{1}, \quad \widehat{\gamma}_{4}\left(\mathbf{e}_{\omega}^{\prime}\right)=-\sqrt{-1} \mathbf{e}_{\omega}^{\prime} .
$$

The combined conditions $\widehat{\gamma}_{4}\left(\mathcal{M}_{1}^{\prime}\right) \subseteq \mathcal{M}_{1}^{\prime}$ and $\phi_{1}^{\prime} \circ \widehat{\gamma}_{4}=\widehat{\gamma}_{4} \circ \phi_{1}^{\prime}$ on $\mathcal{M}_{1}^{\prime}$ are equivalent to

$$
h(u) \equiv-h(-\sqrt{-1} u) \bmod u^{18} .
$$

Since $\left\{u^{6} t-\delta u^{2} t^{3} \mid t \in \mathbf{F}_{3}[u] / u^{36}\right\}$ contains $u^{6}-\delta u^{2}$ and all multiples of $u^{9}$, we may change $\mathbf{e}_{\omega}^{\prime}$ (at the expense of possibly losing the diagonal form for $\widehat{\gamma}_{4}$ ) so that $h=c u^{2}$ for some $c \in \mathbf{F}_{3}$. Since $h$ is necessarily non-zero, we may rescale to get $h=u^{2}$, so there is indeed at most one possibility for the underlying Breuil module $\mathcal{M}$ when $(r, s)=(2,6)$.

Again we treat the cases $\delta= \pm 1$ separately. Consider first the case $\delta=-1$. We have seen above that there is an extension $\mathcal{E}_{6,6}=\left(\mathcal{N}^{\prime},\left\{\widehat{g}^{\prime}\right\}\right)$ of $\mathcal{M}_{6, \omega}^{\prime}$ by $\mathcal{M}_{6,1}^{\prime}$ in 
${\underline{\phi_{1} D D}}_{F^{\prime} / \mathbf{Q}_{3}}$ corresponding to a non-split, peu ramifié extension of 1 by $\omega$. Pulling back $\mathcal{E}_{6,6}$ by a non-zero map

$$
\mathcal{M}_{2, \omega}^{\prime} \longrightarrow \mathcal{M}_{6, \omega}^{\prime}
$$

in ${\underline{\phi_{1} D D}}_{F^{\prime} / \mathbf{Q}_{3}}$ given by $\mathbf{e} \mapsto \pm u^{6} \mathbf{e}$, we get an extension $\mathcal{E}_{2,6}$ of $\mathcal{M}_{2, \omega}^{\prime}$ by $\mathcal{M}_{6,1}^{\prime}$ in $\underline{\phi}_{1} \overline{D D} F^{\prime} / \mathbf{Q}_{3}$ corresponding to a non-split, peu ramifié extension of 1 by $\omega$. The underlying Breuil module of $\mathcal{E}_{2,6}$ must be isomorphic to $\mathbf{F}_{9} \otimes_{\mathbf{F}_{3}} \mathcal{M}$ for our uniquely determined $\mathcal{M}$ (with $h=u^{2}$ ). By the injectivity of $H^{1}\left(G_{3}, \omega\right) \rightarrow H^{1}\left(G_{F^{\prime}}, \omega\right)$ in the $\delta=-1$ case, we conclude that $\mathbf{F}_{9} \otimes_{\mathbf{F}_{3}} \mathcal{N}$ cannot admit descent data giving rise to a très ramifié element in $\operatorname{Ext}_{\mathbf{F}_{3}\left[G_{3}\right]}^{1}(1, \omega)$. This rules out the case $(r, s)=(2,6)$ and $\delta=-1$.

Now turn to the case $(r, s)=(2,6)$ and $\delta=1$. We will show that with the Breuil module $\mathcal{M}$ constructed above (with $h=u^{2}$ ), the Breuil module $\mathcal{M}^{\prime}=\mathbf{F}_{9} \otimes_{\mathbf{F}_{3}} \mathcal{M}$ does not admit descent data relative to $F^{\prime} / \mathbf{Q}_{3}$ (with $\widehat{\gamma}_{2}=\gamma_{2} \otimes 1$, without loss of generality). One checks that $N\left(\mathbf{e}_{1}\right)=N\left(\mathbf{e}_{\omega}^{\prime}\right)=0$, so

$$
N \circ \phi_{1}=0 .
$$

We must have

$$
\widehat{\gamma}_{3}\left(\mathbf{e}_{1}\right)=H_{\gamma_{3}}(u)^{-9} \mathbf{e}_{1}, \quad \widehat{\gamma}_{3}\left(\mathbf{e}_{\omega}^{\prime}\right)=H_{\gamma_{3}}(u)^{-3} \mathbf{e}_{\omega}^{\prime}+h_{\gamma_{3}}(u) \mathbf{e}_{1}
$$

for some $h_{\gamma_{3}} \in \mathbf{F}_{9}[u] / u^{36}$. As usual, since $\widehat{\gamma}_{3}$ and $\widehat{\gamma}_{2}$ must commute, we have $h_{\gamma_{3}} \in \mathbf{F}_{3}[u] / u^{36}$. The condition $\widehat{\gamma}_{3}\left(\mathcal{M}_{1}^{\prime}\right) \subseteq \mathcal{M}_{1}^{\prime}$ is equivalent to

$$
\widehat{\gamma}_{3}\left(u^{2} \mathbf{e}_{\omega}^{\prime}+u^{2} \mathbf{e}_{1}\right) \in \mathcal{M}_{1}^{\prime},
$$

which amounts to

$$
h_{\gamma_{3}}(u) \equiv H_{\gamma_{3}}^{-3}-H_{\gamma_{3}}^{-9} \equiv 0 \bmod u^{4},
$$

so

$$
\widehat{\gamma}_{3}\left(u^{2} \mathbf{e}_{\omega}^{\prime}+u^{2} \mathbf{e}_{1}\right)=H_{\gamma_{3}}(u)^{-1}\left(u^{2} \mathbf{e}_{\omega}^{\prime}+u^{2} \mathbf{e}_{1}\right)+\left(\frac{h_{\gamma_{3}}-H_{\gamma_{3}}^{-3}+H_{\gamma_{3}}^{-9}}{u^{4}}\right) H_{\gamma_{3}}^{2} u^{6} \mathbf{e}_{1} .
$$

As $N \circ \phi_{1}=0$, we have

$$
\widehat{\gamma}_{3} \circ \phi_{1}^{\prime}=\phi_{1}^{\prime} \circ \widehat{\gamma}_{3}
$$

on $\mathcal{M}_{1}^{\prime}$. Evaluating this identity on $u^{2} \mathbf{e}_{\omega}^{\prime}+u^{2} \mathbf{e}_{1} \in \mathcal{M}_{1}$ gives

$$
h_{\gamma_{3}}=H_{\gamma_{3}}^{6} \cdot\left(\frac{h_{\gamma_{3}}-H_{\gamma_{3}}^{-3}+H_{\gamma_{3}}^{-9}}{u^{4}}\right)^{3}
$$

so $h_{\gamma_{3}}$ is a cube. Thus, $h_{\gamma_{3}}=u^{6} g_{\gamma_{3}}$ for some $g_{\gamma_{3}} \in \mathbf{F}_{3}[u] / u^{36}$.

Since $H_{\gamma_{3}}^{3} \equiv 1+u^{6} \bmod u^{12}$, we compute

$$
H_{\gamma_{3}}^{-9}-H_{\gamma_{3}}^{-3} \equiv u^{6} \bmod u^{12},
$$

so

$$
h_{\gamma_{3}} \equiv H_{\gamma_{3}}^{6} \cdot\left(\left(\frac{h_{\gamma_{3}}}{u^{4}}\right)^{3}+u^{6}\right) \bmod u^{7}
$$

and

$$
g_{\gamma_{3}}(0)=g_{\gamma_{3}}(0)^{3}+1
$$


in $\mathbf{F}_{3}$. This is absurd. This rules out all possibilities for $(r, s)$ aside from $(r, s)=$ $(2,10)$. Uniqueness now follows from Corollary 4.1.5

From Theorem 5.4.2 of [Man] and Proposition B.4.2 of [CDT] we see that there is an elliptic curve $E_{/ \mathbf{Q}_{3}}$ such that $E[3]\left(\overline{\mathbf{Q}}_{3}\right) \cong \bar{\rho}$ and $\rho_{E, 3}$ has type $\tau_{ \pm 3}$. Let $\mathcal{E}$ denote the Néron model of $E \times{ }_{\mathbf{Q}_{3}} F^{\prime}$ over $\mathcal{O}_{F^{\prime}}$. By the Néron property of $\mathcal{E}_{/ \mathcal{O}_{F^{\prime}}}$ we see that $\mathcal{E}\left[3^{\infty}\right]$ has descent data over $\mathbf{Q}_{3}$. As in $\$ 4.5$ we see that $\mathcal{J}$ annihilates the Dieudonné module of $\mathcal{E}\left[3^{\infty}\right] \times \mathbf{F}_{9}$. Thus $\mathcal{M}(\bar{\rho})^{\prime} \cong \mathcal{M}_{\pi}(\mathcal{E}[3])$ in $\underline{\phi}_{1} D D_{F^{\prime} / \mathbf{Q}_{3}}$ and it follows that Frobenius is non-zero on $D\left(\mathcal{G}^{\prime}\right)$.

\subsection{Completion of the proof of Theorem 4.5.1.}

Lemma 8.3.1. Let $\left(\mathcal{G}^{\prime},\{[g]\}\right)$ be the unique object of $\mathcal{F D}_{F^{\prime} / \mathbf{Q}_{3}, \mathcal{J}}$ such that $\left(\mathcal{G}^{\prime},\{[g]\}\right)_{\mathbf{Q}_{3}}$ corresponds to $\bar{\rho}$. Set $\left(\mathcal{M}(\bar{\rho})^{\prime},\{\widehat{g}\}\right)=\mathcal{M}_{\pi}\left(\mathcal{G}^{\prime},\{[g]\}\right)$. The natural map of groups

$$
\operatorname{Ext}_{\underline{\phi_{1} D D}}^{1}{ }_{F^{\prime} / \mathbf{Q}_{3}}\left(\left(\mathcal{M}(\bar{\rho})^{\prime},\{\widehat{g}\}\right),\left(\mathcal{M}(\bar{\rho})^{\prime},\{\widehat{g}\}\right)\right) \longrightarrow \operatorname{Ext}_{\underline{\phi_{1} D D}}^{1}{ }_{F^{\prime} / \mathbf{Q}_{3}}\left(\mathcal{M}_{10,1}^{\prime}, \mathcal{M}_{2, \omega}^{\prime}\right),
$$

using pushout by $\left(\mathcal{M}(\bar{\rho})^{\prime},\{\widehat{g}\}\right) \rightarrow \mathcal{M}_{2, \omega}^{\prime}$ and pullback by $\mathcal{M}_{10,1}^{\prime} \rightarrow\left(\mathcal{M}(\bar{\rho})^{\prime},\{\widehat{g}\}\right)$, is zero.

Proof. Let $\left(\widetilde{\mathcal{M}}^{\prime},\{\widehat{g}\}\right)$ represent a class in $\operatorname{Ext}_{\underline{\phi_{1} D D^{\prime}}}^{1}{ }_{F^{\prime} / \mathbf{Q}_{3}}\left(\left(\mathcal{M}(\bar{\rho})^{\prime},\{\widehat{g}\}\right),\left(\mathcal{M}(\bar{\rho})^{\prime},\{\widehat{g}\}\right)\right)$ and let $\left(\mathcal{M}^{\prime},\{\widehat{g}\}\right)$ be its image in $\operatorname{Ext}_{\underline{\phi_{1} D D_{F^{\prime} / \mathbf{Q}_{3}}}}^{1}\left(\mathcal{M}_{10, \mathbf{1}}^{\prime}, \mathcal{M}_{2, \omega}^{\prime}\right)$. By Lemma 5.2.2 $\mathcal{M}^{\prime}=\mathbf{F}_{9} \otimes \mathcal{M}$ with $\widehat{\gamma}_{2}=\gamma_{2} \otimes 1$ and

$$
\mathcal{M}=\left(\mathbf{F}_{3}[u] / u^{36}\right) \mathbf{e}_{\omega} \oplus\left(\mathbf{F}_{3}[u] / u^{36}\right) \mathbf{e}_{1}^{\prime}, \quad \mathcal{M}_{1}=\left\langle u^{2} \mathbf{e}_{\omega}, u^{10} \mathbf{e}_{1}^{\prime}+\left(c+c^{\prime} u\right) \mathbf{e}_{\omega}\right\rangle,
$$

with $c, c^{\prime} \in \mathbf{F}_{3}$. Also,

$$
\phi_{1}\left(u^{2} \mathbf{e}_{\omega}\right)=\mathbf{e}_{\omega}, \quad \phi_{1}\left(u^{10} \mathbf{e}_{1}^{\prime}+\left(c+c^{\prime} u\right) \mathbf{e}_{\omega}\right)=\delta \mathbf{e}_{1}^{\prime},
$$

and

$$
\widehat{\gamma}_{4}\left(\mathbf{e}_{\omega}\right)=-\sqrt{-1} \mathbf{e}_{\omega}, \quad \widehat{\gamma}_{4}\left(\mathbf{e}_{1}^{\prime}\right)=\sqrt{-1} \mathbf{e}_{1}^{\prime}+h_{\gamma_{4}}(u) \mathbf{e}_{\omega}
$$

for some $h_{\gamma_{4}} \in \mathbf{F}_{9}[u] / u^{36}$.

The properties $\widehat{\gamma}_{4}\left(\mathcal{M}_{1}^{\prime}\right) \subseteq \mathcal{M}_{1}^{\prime}$ and $\widehat{\gamma}_{4} \circ \phi_{1}^{\prime}=\phi_{1}^{\prime} \circ \widehat{\gamma}_{4}$ on $\mathcal{M}_{1}^{\prime}$ amount to

$$
c^{\prime}=0, \quad h_{\gamma_{4}}=-\delta h_{\gamma_{4}}^{3} u^{24}
$$

so $h_{\gamma_{4}}=0$. If $c=0$, then $N \circ \phi_{1}=0$, so $\widehat{\gamma}_{3} \circ \phi_{1}^{\prime}=\phi_{1}^{\prime} \circ \widehat{\gamma}_{3}$ on $\mathcal{M}_{1}^{\prime}$. From this we

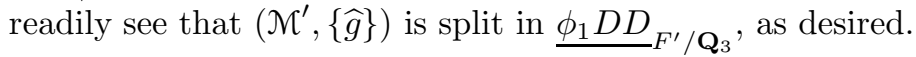

Now assume $c \neq 0$; we will deduce a contradiction. Consider the rank three Breuil module with descent data

$$
(\mathcal{N},\{\widehat{g}\})=\left(\widetilde{\mathcal{M}}^{\prime},\{\widehat{g}\}\right) /\left(\mathcal{M}_{10,1}^{\prime},\{\widehat{g}\}\right),
$$

where $\mathcal{M}_{10, \mathbf{1}}^{\prime} \hookrightarrow \mathcal{M}(\bar{\rho})^{\prime} \hookrightarrow \widetilde{\mathcal{M}}^{\prime}$. Then $\mathcal{N}$ has an ordered basis $\left\{\mathbf{e}_{\omega}, \mathbf{e}_{1}^{\prime}, \mathbf{e}_{\omega}^{\prime}\right\}$ with respect to which

$$
\mathcal{N}_{1}=\left\langle u^{2} \mathbf{e}_{\omega}, u^{10} \mathbf{e}_{1}^{\prime}+\mathbf{e}_{\omega}, u^{2} \mathbf{e}_{\omega}^{\prime}+h \mathbf{e}_{1}^{\prime}+\left(b+b^{\prime} u\right) \mathbf{e}_{\omega}\right\rangle
$$

for some $b, b^{\prime} \in \mathbf{F}_{9}$ and $h=a+a^{\prime} u^{4}+a^{\prime \prime} u^{8} \in \mathbf{F}_{9}[u] /\left(u^{36}\right)$ defined modulo $\left\{u^{10} t-\right.$ $\left.\delta u^{2} t^{3}\right\}$ (see 8.2.1). Since our base field $F^{\prime}$ has absolute ramification degree $12, \mathcal{N}_{1}$ contains

$$
u^{12} \mathbf{e}_{\omega}^{\prime}=u^{10}\left(u^{2} \mathbf{e}_{\omega}^{\prime}+h \mathbf{e}_{1}^{\prime}+\left(b+b^{\prime} u\right) \mathbf{e}_{\omega}\right)-h\left(u^{10} \mathbf{e}_{1}^{\prime}+\mathbf{e}_{\omega}\right)+\left(h-u^{10}\left(b+b^{\prime} u\right)\right) \mathbf{e}_{\omega} .
$$


From the list of generators of $\mathcal{N}_{1}$, it is not difficult to check that in the above expression for $u^{12} \mathbf{e}_{\omega}^{\prime} \in \mathcal{N}_{1}, u^{2}$ must divide the coefficient of $\mathbf{e}_{\omega}$. Thus $a=0$.

We must have $\mathcal{N} /\left\langle\mathbf{e}_{\omega}\right\rangle \cong \mathcal{M}(\bar{\rho})^{\prime}$. Since $a=0, \mathcal{M}(\bar{\rho})$ has basis $\left\{\mathbf{e}_{1}^{\prime}, \mathbf{e}_{\omega}^{\prime}\right\}$ and

$$
\mathcal{M}(\bar{\rho})_{1}=\left\langle u^{10} \mathbf{e}_{1}^{\prime}, u^{2} \mathbf{e}_{\omega}^{\prime}+\left(a^{\prime} u^{4}+a^{\prime \prime} u^{8}\right) \mathbf{e}_{1}^{\prime}\right\rangle
$$

Since $\phi_{1}$ for $\mathcal{M}(\bar{\rho})$ satisfies

$$
\phi_{1}\left(u^{10} \mathbf{e}_{1}^{\prime}\right)=\delta \mathbf{e}_{1}^{\prime}, \quad \phi_{1}\left(u^{2} \mathbf{e}_{\omega}^{\prime}+\left(a^{\prime} u^{4}+a^{\prime \prime} u^{8}\right) \mathbf{e}_{1}^{\prime}\right)=\mathbf{e}_{\omega}^{\prime},
$$

it follows immediately that $\phi \equiv 0 \bmod u \mathcal{N}(\bar{\rho})$, which (using Theorem 5.1.3) contradicts Proposition 8.2.1.

Corollary 8.3.2. The natural map

$$
\theta_{0}: \operatorname{Ext}_{\delta_{ \pm 3}}^{1}(\bar{\rho}, \bar{\rho}) \longrightarrow H^{1}\left(G_{3}, \omega\right)
$$

is zero.

Theorem 4.7.4 and hence Theorem 4.4.1 now follow from the first case of the following lemma. We include the second case to simplify the proof.

Lemma 8.3.3. The maps of groups

$$
\begin{gathered}
\operatorname{Ext}_{\frac{\phi_{1} D D_{F^{\prime} / \mathbf{Q}_{3}}}{1}}^{1}\left(\mathcal{M}_{10, \mathbf{1}}^{\prime}, \mathcal{M}_{10, \mathbf{1}}^{\prime}\right) \longrightarrow \operatorname{Ext}_{\mathbf{F}_{3}\left[G_{3}\right]}^{1}(1,1), \\
\operatorname{Ext}_{\underline{\phi_{1} D D} F_{F^{\prime} / \mathbf{Q}_{3}}}^{1}\left(\mathcal{M}_{2, \omega}^{\prime}, \mathcal{M}_{2, \omega}^{\prime}\right) \longrightarrow \operatorname{Ext}_{\mathbf{F}_{3}\left[G_{3}\right]}^{1}(\omega, \omega)
\end{gathered}
$$

have images inside the line of extension classes that split over an unramified extension of $\mathbf{Q}_{3}$.

Proof. Since

$$
H^{1}\left(G_{3}, \mathbf{Z} / 3\right) \longrightarrow H^{1}\left(G_{F^{\prime}}, \mathbf{Z} / 3\right)
$$

is injective and induces an isomorphism between the subgroups of unramified classes, it suffices to check that

$$
\begin{gathered}
\operatorname{Ext}_{\underline{\phi_{1} D D}}^{1}{ }_{F^{\prime} / \mathbf{Q}_{3}}\left(\mathcal{M}_{10, \mathbf{1}}^{\prime}, \mathcal{M}_{10, \mathbf{1}}^{\prime}\right) \longrightarrow \operatorname{Ext}_{{\underline{\phi_{1}-\bmod _{F}}}_{F}^{1}}^{1}(1,1), \\
\operatorname{Ext}_{{\underline{\phi_{1} D D}}_{F^{\prime} / \mathbf{Q}_{3}}^{1}}^{1}\left(\mathcal{M}_{2, \omega}^{\prime}, \mathcal{M}_{2, \omega}^{\prime}\right) \longrightarrow \operatorname{Ext}_{{\underline{\phi_{1}-\bmod _{F}}}_{F}}^{1}(\omega, \omega)
\end{gathered}
$$

have images consisting of elements split over an unramified extension of $F$. By Cartier duality it suffices to consider only the second map.

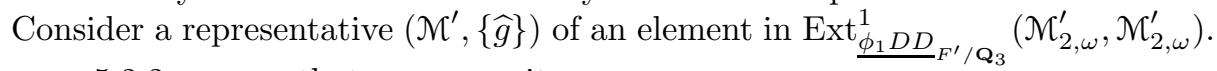
Lemma 5.2.2 ensures that we can write

$$
\mathcal{M}=\left(\mathbf{F}_{3}[u] / u^{36}\right) \mathbf{e}_{\omega} \oplus\left(\mathbf{F}_{3}[u] / u^{36}\right) \mathbf{e}_{\omega}^{\prime}, \quad \mathcal{M}_{1}=\left\langle u^{2} \mathbf{e}_{\omega}, u^{2} \mathbf{e}_{\omega}^{\prime}+h \mathbf{e}_{\omega}\right\rangle
$$

for some $h=c+c^{\prime} u+c^{\prime \prime} u^{2}$ with $c, c^{\prime}, c^{\prime \prime} \in \mathbf{F}_{3}$ and

$$
\phi_{1}\left(u^{2} \mathbf{e}_{\omega}\right)=\mathbf{e}_{\omega}, \quad \phi_{1}\left(u^{2} \mathbf{e}_{\omega}^{\prime}+h \mathbf{e}_{\omega}\right)=\mathbf{e}_{\omega}^{\prime} .
$$

We have

$$
\widehat{\gamma}_{4}\left(\mathbf{e}_{\omega}\right)=-\sqrt{-1} \mathbf{e}_{\omega}, \quad \widehat{\gamma}_{4}\left(\mathbf{e}_{\omega}^{\prime}\right)=-\sqrt{-1} \mathbf{e}_{\omega}^{\prime}+h_{\gamma_{4}}(u) \mathbf{e}_{\omega}
$$

for some $h_{\gamma_{4}} \in \mathbf{F}_{9}[u] / u^{36}$, and the condition $\widehat{\gamma}_{4}\left(\mathcal{M}_{1}^{\prime}\right) \subseteq \mathcal{M}_{1}^{\prime}$ is equivalent to

$$
h(u) \equiv-h(-\sqrt{-1} u) \bmod u^{2},
$$


so $c=c^{\prime}=0$. The Breuil module extension class $\mathcal{M}$ over $\mathcal{O}_{F}$ (ignoring descent data) therefore only depends on the parameter $c^{\prime \prime} \in \mathbf{F}_{3}$. We then have a splitting $\overline{\mathbf{F}}_{3} \otimes_{\mathbf{F}_{3}} \mathcal{M}(2,1) \rightarrow \overline{\mathbf{F}}_{3} \otimes_{\mathbf{F}_{3}} \mathcal{M}$ determined by

$$
\mathbf{e} \longmapsto a \mathbf{e}_{\omega}+\mathbf{e}_{\omega}^{\prime},
$$

where $a \in \overline{\mathbf{F}}_{3}$ satisfies $a^{3}=a+c^{\prime \prime}$.

\section{Proof of Theorems 4.6.1, 4.6.2 And 4.6.3}

In this section we will keep the notation of $\$ 4.6$ and $\$ 6.5$. We will write $F$ for $F_{i}, F^{\prime}$ for $F_{i}^{\prime}$ and $\mathcal{J}$ for $\mathcal{J}_{i}$. If $\mathcal{G}$ (resp. $\mathcal{M}$ ) is a finite flat $\mathcal{O}_{F}$-group scheme (resp. Breuil module over $\mathcal{O}_{F}$ ) we will write $\mathcal{G}^{\prime}\left(\right.$ resp. $\mathcal{M}^{\prime}$ ) for the base change to $\mathcal{O}_{F^{\prime}}$.

9.1. Rank one calculations. We remark that with our choice of polynomials $H_{g}(u)$ in 6.5 any object $\mathcal{M}$ in $\underline{\phi}_{1} D D_{F^{\prime} / \mathbf{Q}_{3}}$ has an action of $\left\langle\gamma_{2}, \gamma_{4}\right\rangle$ via $\widehat{\gamma}_{2}$ and $\widehat{\gamma}_{4}$.

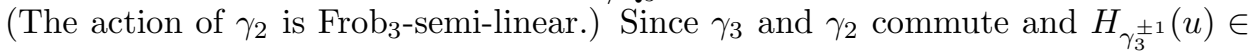
$\mathbf{Z}_{3}[u]$ we see that $\widehat{\gamma}_{2}$ must commute with $\widehat{\gamma_{3}^{ \pm 1}}$ (see Corollary $\widehat{5.6 .2}$ ).

By Lemma 5.2.1 the only models for $(\mathbf{Z} / 3 \mathbf{Z})_{/ F}$ over $\mathcal{O}_{F}$ are $\mathcal{G}(r, 1)$ for $r=$ $0,2,4,6,8,10,12$ with $\mathcal{G}(12,1) \cong(\mathbf{Z} / 3 \mathbf{Z})_{/ \mathcal{O}_{F}}$, and the only models for $\left(\mu_{3}\right)_{/ F}$ over $\mathcal{O}_{F}$ are $\mathcal{G}(r, 1)$ for $r=0,2,4,6,8,10,12$ with $\mathcal{G}(0,1) \cong\left(\mu_{3}\right) / \mathcal{O}_{F}$. Lemma 5.7.1 ensures that the base changes to $\mathcal{O}_{F^{\prime}}$ admit unique descent data over $\mathbf{Q}_{3}$ such that descent of the generic fibre to $\mathbf{Q}_{3}$ is $\mathbf{Z} / 3 \mathbf{Z}$ (resp. $\mu_{3}$ ). We will write $\mathcal{G}_{r, 1}^{\prime}$ (resp. $\mathcal{G}_{r, \omega}^{\prime}$ ) for the corresponding pair $\left(\mathcal{G}(r, 1) \times \times_{\mathcal{O}_{F}} \mathcal{O}_{F^{\prime}},\{[g]\}\right)\left(\operatorname{resp} .\left(\mathcal{G}(r, 1) \times_{\mathcal{O}_{F}} \mathcal{O}_{F^{\prime}},\{[g]\}\right)\right)$. We will also let $\mathcal{M}_{r, 1}^{\prime}$ (resp. $\mathcal{M}_{r, \omega}^{\prime}$ ) denote the corresponding object of $\underline{\phi_{1} D D}{ }_{F^{\prime}} / \mathbf{Q}_{3}$.

We have the following useful lemmas, for which the proofs are identical to the proofs of Lemmas 8.1 .1 and 8.1 .2 .

Lemma 9.1.1. Let $0 \leq r \leq e=12$ be an even integer. The descent data on $\mathcal{M}_{r, 1}$ is determined by

$$
\widehat{\gamma_{2}}(\mathbf{e})=\mathbf{e}, \widehat{\gamma_{4}}(\mathbf{e})=-(-\sqrt{-1})^{r / 2} \mathbf{e}, \widehat{\gamma_{3}^{ \pm 1}}(\mathbf{e})=H_{\gamma_{3}^{ \pm 1}}(u)^{-3 r / 2} \mathbf{e},
$$

and the descent data on $\mathcal{M}_{r, \omega}$ is determined by

$$
\widehat{\gamma_{2}}(\mathbf{e})=\mathbf{e}, \widehat{\gamma_{4}}(\mathbf{e})=(-\sqrt{-1})^{r / 2} \mathbf{e}, \widehat{\gamma_{3}^{ \pm 1}}(\mathbf{e})=H_{\gamma_{3}^{ \pm 1}}(u)^{-3 r / 2} \mathbf{e} .
$$

In particular, $\gamma_{4}^{2}=-1$ on $D\left(\mathcal{G}_{r, 1}\right)$ if and only if $\gamma_{4}^{2}=-1$ on $D\left(\mathcal{G}_{r, \omega}\right)$ if and only if $r=2,6$ or 10 .

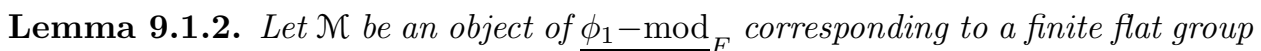
scheme $\mathcal{G}$ and let $\{[g]\}$ be descent data on $\mathcal{G}^{\prime}=\mathcal{G} \times{ }_{\mathcal{O}_{F}} \mathcal{O}_{F^{\prime}}$ over $\mathbf{Q}_{3}$. Assume that $\left(\mathcal{G}^{\prime},\{\widehat{g}\}\right)_{\mathbf{Q}_{3}}$ can be filtered so that each graded piece is isomorphic to $\mathbf{Z} / 3 \mathbf{Z}$ or $\mu_{3}$ and so that the corresponding filtration of $\left(\mathcal{M}^{\prime},\{\widehat{g}\}\right)$ in $\underline{\phi}_{1} D D F_{F^{\prime} / \mathbf{Q}_{3}}$ has successive quotients of the form $\mathcal{M}_{r_{j}, \chi_{j}}^{\prime}$ with $r_{j} \in\{2,6,10\}$ and $\chi_{j} \in\{\mathbf{1}, \omega\}$. Then $\gamma_{4}^{2}=-1$ on $\mathcal{M}^{\prime} / u \mathcal{M}^{\prime}$ and there exists a basis $\left\{\mathbf{e}_{j}\right\}$ of $\mathcal{M}$ over $\mathbf{F}_{3}[u] / u^{36}$ so that for all $j$

- $\mathbf{e}_{j} \in \phi_{1}\left(\mathcal{M}_{1}\right)$,

- $\mathbf{e}_{j}$ is an eigenvector of the $\mathbf{F}_{9}$-linear map $\widehat{\gamma_{4}}$ on $\mathcal{M}^{\prime}$,

- $\mathbf{e}_{j}$ lies in the part of the filtration of $\mathcal{N}^{\prime}$ which surjects onto $\mathcal{M}_{r_{j}, \chi_{j}}^{\prime}$ and this surjection sends $\mathbf{e}_{j}$ onto the standard basis vector $\mathbf{e}$ of $\mathcal{M}_{r_{j}, \chi_{j}}^{\prime}$ over $\mathbf{F}_{9}[u] / u^{36}$. 
9.2. Models for $\bar{\rho}$. Recall that we are assuming that $\bar{\rho}$ has the très ramifié form

$$
\left(\begin{array}{cc}
\omega & * \\
0 & 1
\end{array}\right)
$$

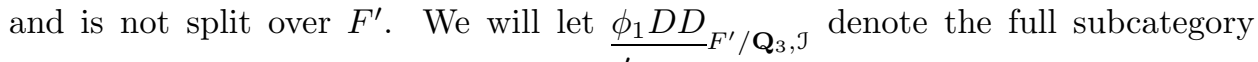
of $\underline{\phi}_{1} D D F_{F^{\prime} / \mathbf{Q}_{3}}$ consisting of objects $\mathcal{M}^{\prime}$ for which the ideal $\mathcal{J}$ acts trivially on $\left(\mathcal{M}^{\prime} / u \mathcal{M}^{\prime}\right) \otimes_{\mathbf{F}_{9}, \mathrm{Frob}_{3}} \mathbf{F}_{9}$.

Proposition 9.2.1. Suppose that $\left(\mathcal{M}^{\prime},\{\widehat{g}\}\right)$ is an object of ${\underline{\phi_{1} D D}}_{F^{\prime} / \mathbf{Q}_{3}, \mathcal{J}}$ such that $\left(\mathcal{M}^{\prime},\{\widehat{g}\}\right)_{\mathbf{Q}_{3}}$ is an extension of $\mathbf{Z} / 3 \mathbf{Z}$ by $\mu_{3}$. Then we have an exact sequence

$$
(0) \longrightarrow \mathcal{M}_{s, 1}^{\prime} \longrightarrow \mathcal{M}^{\prime} \longrightarrow \mathcal{M}_{r, \omega}^{\prime} \longrightarrow(0)
$$

with $(r, s)=(2,6),(6,10),(2,10)$ or $(6,6)$. Moreover we can write $\mathcal{M}^{\prime}=\mathcal{M} \otimes_{\mathbf{F}_{3}} \mathbf{F}_{9}$ with $\widehat{\gamma}_{2}=1 \otimes$ Frob $_{3}$, where $\mathcal{M}$ has an $\mathbf{F}_{3}[u] /\left(u^{36}\right)$-basis $\left\{\mathbf{e}_{1}, \mathbf{e}_{\omega}^{\prime}\right\}$ with $\mathbf{e}_{1}$ the standard basis element of $\mathcal{M}(s, 1)$ and $\mathbf{e}_{\omega}^{\prime}$ mapping to the standard basis element of $\mathcal{M}(r, 1)$. More precisely we have the following exhaustive list of extension class possibilities, all of which are well defined. ( $N$ denotes the monodromy operator described in Lemma 5.1.2.)

(1) $(r, s)=(2,6):$ The natural map

$$
\operatorname{Ext}_{\underline{\phi_{1} D D}}^{1}{ }_{F^{\prime} / \mathbf{Q}_{3}, j}\left(\mathcal{M}_{2, \omega}^{\prime}, \mathcal{M}_{6, \mathbf{1}}^{\prime}\right) \longrightarrow \operatorname{Ext}_{\mathbf{F}_{3}\left[G_{3}\right]}^{1}(1, \omega)
$$

is an isomorphism, with elements parametrised by pairs $\left(c, c_{1}\right) \in \mathbf{F}_{3}^{2}$ corresponding to

$$
\begin{aligned}
& \mathcal{M}_{1}=\left\langle u^{6} \mathbf{e}_{1}, u^{2} \mathbf{e}_{\omega}^{\prime}+c u^{2} \mathbf{e}_{1}\right\rangle, \phi_{1}\left(u^{6} \mathbf{e}_{1}\right)=\mathbf{e}_{1}, \phi_{1}\left(u^{2} \mathbf{e}_{\omega}^{\prime}+c u^{2} \mathbf{e}_{1}\right)=\mathbf{e}_{\omega}^{\prime} \\
& \left(\text { so } N \circ \phi_{1}=0\right) \text { with }
\end{aligned}
$$

$$
\begin{gathered}
\widehat{\gamma}_{4}\left(\mathbf{e}_{1}\right)=-\sqrt{-1} \mathbf{e}_{1}, \quad \widehat{\gamma}_{4}\left(\mathbf{e}_{\omega}^{\prime}\right)=-\sqrt{-1} \mathbf{e}_{\omega}^{\prime}, \\
\widehat{\gamma_{3}^{ \pm 1}}\left(\mathbf{e}_{1}\right)=\mathbf{e}_{1}, \widehat{\gamma_{3}^{ \pm 1}}\left(\mathbf{e}_{\omega}^{\prime}\right)=\left(1 \pm u^{18}\right)\left(\mathbf{e}_{\omega}^{\prime} \pm c_{1} u^{6} \mathbf{e}_{1}\right) .
\end{gathered}
$$

The pairs with $c=0$ are the ones which generically split over $F^{\prime}$. In all cases $\phi \equiv 0 \bmod u \mathcal{N}$.

(2) $\underline{(r, s)=(6,10)}$ : The natural map

$$
\operatorname{Ext}_{\underline{\phi_{1} D D}}^{1}{ }_{F^{\prime} / \mathbf{Q}_{3}, j}\left(\mathcal{M}_{6, \omega}^{\prime}, \mathcal{M}_{10, \mathbf{1}}^{\prime}\right) \longrightarrow \operatorname{Ext}_{\mathbf{F}_{3}\left[G_{3}\right]}^{1}(1, \omega)
$$

is an isomorphism, with elements parametrised by pairs $\left(c, c_{1}\right) \in \mathbf{F}_{3}^{2}$ corresponding to

$$
\begin{gathered}
\mathcal{M}_{1}=\left\langle u^{10} \mathbf{e}_{1}, u^{6} \mathbf{e}_{\omega}^{\prime}+c u^{6} \mathbf{e}_{1}\right\rangle, \quad \phi_{1}\left(u^{10} \mathbf{e}_{1}\right)=\mathbf{e}_{1}, \quad \phi_{1}\left(u^{6} \mathbf{e}_{\omega}^{\prime}+c u^{6} \mathbf{e}_{1}\right)=\mathbf{e}_{\omega}^{\prime} \\
\left(\text { so } N \circ \phi_{1}=0\right) \text { with } \\
\widehat{\gamma}_{4}\left(\mathbf{e}_{1}\right)=\sqrt{-1} \mathbf{e}_{1}, \widehat{\gamma}_{4}\left(\mathbf{e}_{\omega}^{\prime}\right)=\sqrt{-1} \mathbf{e}_{\omega}^{\prime}, \\
\widehat{\gamma_{3}^{ \pm 1}}\left(\mathbf{e}_{1}\right)=\left(1 \mp u^{18}\right) \mathbf{e}_{1}, \widehat{\gamma_{3}^{ \pm 1}}\left(\mathbf{e}_{\omega}^{\prime}\right)=\mathbf{e}_{\omega}^{\prime} \pm c_{1} u^{6} \mathbf{e}_{1} .
\end{gathered}
$$

The pairs with $c=0$ are the ones which generically split over $F^{\prime}$. In all cases $\phi \equiv 0 \bmod u \mathcal{M}$. These cases are Cartier dual to the $(2,6)$ cases above. 
(3) $(r, s)=(2,10)$ : The natural map

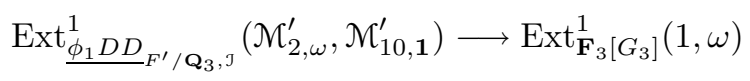

is an isomorphism, with elements parametrised by pairs $\left(c, c_{1}\right) \in \mathbf{F}_{3}^{2}$ corresponding to

$$
\begin{aligned}
& \mathcal{M}_{1}=\left\langle u^{10} \mathbf{e}_{1}, u^{2} \mathbf{e}_{\omega}^{\prime}+c u^{8} \mathbf{e}_{1}\right\rangle, \phi_{1}\left(u^{10} \mathbf{e}_{1}\right)=\mathbf{e}_{1}, \phi_{1}\left(u^{2} \mathbf{e}_{\omega}^{\prime}+c u^{8} \mathbf{e}_{1}\right)=\mathbf{e}_{\omega}^{\prime} \\
& \left(\text { so } N \circ \phi_{1}=0\right) \text { with }
\end{aligned}
$$

$$
\begin{gathered}
\widehat{\gamma}_{4}\left(\mathbf{e}_{1}\right)=\sqrt{-1} \mathbf{e}_{1}, \quad \widehat{\gamma}_{4}\left(\mathbf{e}_{\omega}^{\prime}\right)=-\sqrt{-1} \mathbf{e}_{\omega}^{\prime}, \\
\widehat{\gamma_{3}^{ \pm 1}}\left(\mathbf{e}_{1}\right)=\left(1 \mp u^{18}\right) \mathbf{e}_{1}, \widehat{\gamma_{3}^{ \pm 1}}\left(\mathbf{e}_{\omega}^{\prime}\right)=\left(1 \pm u^{18}\right)\left(\mathbf{e}_{\omega}^{\prime} \pm c_{1} u^{12} \mathbf{e}_{1}\right) .
\end{gathered}
$$

The pairs with $c=0$ are the ones which generically split over $F^{\prime}$. In all cases $\phi \equiv 0 \bmod u \mathcal{N}$.

(4) $(r, s)=(6,6)$ : The natural map

$$
\operatorname{Ext}_{\underline{\phi_{1} D D}}^{1}{ }_{F^{\prime} / \mathbf{Q}_{3}, j}\left(\mathcal{M}_{6, \omega}^{\prime}, \mathcal{M}_{6,1}^{\prime}\right) \longrightarrow \operatorname{Ext}_{\mathbf{F}_{3}\left[G_{3}\right]}^{1}(1, \omega)
$$

is an isomorphism, with elements parametrised by pairs $\left(c, c^{\prime}\right) \in \mathbf{F}_{3}^{2}$ corresponding to

$$
\mathcal{M}_{1}=\left\langle u^{6} \mathbf{e}_{1}, u^{6} \mathbf{e}_{\omega}^{\prime}+\left(c+c^{\prime} u^{4}\right) \mathbf{e}_{1}\right\rangle, \quad \phi_{1}\left(u^{6} \mathbf{e}_{1}\right)=\mathbf{e}_{1}, \phi_{1}\left(u^{6} \mathbf{e}_{\omega}^{\prime}+\left(c+c^{\prime} u^{4}\right) \mathbf{e}_{1}\right)=\mathbf{e}_{\omega}^{\prime}
$$

(it is easily checked that $N\left(\mathbf{e}_{1}\right)=0$ and $N\left(\mathbf{e}_{\omega}^{\prime}\right)=c^{\prime} u^{30} \mathbf{e}_{1}$ ) and

$$
\begin{gathered}
\widehat{\gamma}_{4}\left(\mathbf{e}_{1}\right)=-\sqrt{-1} \mathbf{e}_{1}, \widehat{\gamma}_{4}\left(\mathbf{e}_{\omega}^{\prime}\right)=\sqrt{-1} \mathbf{e}_{\omega}^{\prime}, \\
\widehat{\gamma_{3}^{ \pm 1}}\left(\mathbf{e}_{1}\right)=\mathbf{e}_{1}, \widehat{\gamma_{3}^{ \pm 1}}\left(\mathbf{e}_{\omega}^{\prime}\right)=\mathbf{e}_{\omega}^{\prime}+\left( \pm c \mp c^{\prime} u^{12}-c^{\prime} u^{30}\right) \mathbf{e}_{1} .
\end{gathered}
$$

In particular, $\phi \equiv 0 \bmod u \mathcal{M}$ if and only if $c=0$.

In the first three cases, the peu ramifié condition on a class in $\operatorname{Ext}_{\mathbf{F}_{3}\left[G_{3}\right]}^{1}(1, \omega)$ is equivalent to the vanishing of $c_{1}$. In the fourth case it is equivalent to the vanishing of $c$.

Proof. By Lemma 9.1.1 we have an exact sequence

$$
(0) \longrightarrow \mathcal{M}_{s, 1}^{\prime} \longrightarrow \mathcal{M}^{\prime} \longrightarrow \mathcal{M}_{r, \omega}^{\prime} \longrightarrow(0)
$$

with $r, s \in\{2,6,10\}$. As usual

$$
\mathcal{M}_{1}=\left\langle u^{s} \mathbf{e}_{1}, u^{r} \mathbf{e}_{\omega}^{\prime}+h \mathbf{e}_{1}\right\rangle .
$$

In the cases $(r, s)=(2,2)$ and $(6,2)$ as in the proof of Proposition 8.2.1 we may take $h=0$. We will show that in the case $(r, s)=(10,2)$ we also have $h=0$. Following the proof of Proposition 8.2.1 we may suppose that $h \in \mathbf{F}_{3}$. Without loss of generality we can take $h=1$ and look for a contradiction. Again following the proof of Proposition 8.2.1 and using

$$
\mathcal{M}_{1}=\left\langle u^{2} \mathbf{e}_{1}, u^{10} \mathbf{e}_{\omega}^{\prime}+\mathbf{e}_{1}\right\rangle
$$

we find that $\phi \mathbf{e}_{\omega}^{\prime} \equiv-\mathbf{e}_{1} \bmod u \mathcal{M}$. Also

$$
\begin{aligned}
\widehat{\gamma_{3}^{ \pm 1}} \mathbf{e}_{1} & =\left(1 \pm u^{18}\right) \mathbf{e}_{1} \\
\widehat{\gamma_{3}^{ \pm 1}} \mathbf{e}_{\omega}^{\prime} & =\left(1 \mp u^{18}\right) \mathbf{e}_{\omega}^{\prime}+h_{ \pm 1}(u) \mathbf{e}_{1}
\end{aligned}
$$


for some $h_{ \pm 1}(u) \in \mathbf{F}_{9}[u] /\left(u^{36}\right)$, which must actually lie in $\mathbf{F}_{3}[u] /\left(u^{36}\right)$ (using, as usual, the fact that $\widehat{\gamma}_{2}$ and $\widehat{\gamma}_{3}$ commute). Thus

$$
\begin{aligned}
-\mathbf{e}_{1} & \equiv \phi \mathbf{e}_{\omega}^{\prime} \\
& \equiv\left(\widehat{\gamma}_{3} \widehat{\gamma}_{2}-\widehat{\gamma_{3}^{-1}} \widehat{\gamma}_{2}\right)\left(\mathbf{e}_{\omega}^{\prime}\right) \\
& \equiv\left(\widehat{\gamma}_{3}-\widehat{\gamma_{3}^{-1}}\right)\left(\mathbf{e}_{\omega}^{\prime}\right) \\
& \equiv\left(h_{1}(0)-h_{-1}(0)\right) \mathbf{e}_{1} \bmod u \mathcal{M}^{\prime}
\end{aligned}
$$

The inverse linear maps $\widehat{\gamma_{3}^{ \pm 1}}$ on $\mathcal{M}^{\prime} / u \mathcal{M}^{\prime}$ have matrices

$$
\left(\begin{array}{cc}
1 & h_{ \pm 1}(0) \\
0 & 1
\end{array}\right)
$$

with respect to the basis $\left\{\mathbf{e}_{1}, \mathbf{e}_{\omega}^{\prime}\right\}$, so that $h_{-1}(0)=-h_{1}(0)$. Thus $h_{1}(0)=1$. On the other hand evaluating $\widehat{\gamma}_{3} \phi_{1}^{\prime} \equiv \phi_{1}^{\prime} \widehat{\gamma}_{3} \bmod u \mathcal{M}^{\prime}$ on $u^{10} \mathbf{e}_{\omega}^{\prime}+\mathbf{e}_{1}$ and comparing coefficients of $\mathbf{e}_{1}$ gives $h_{1}(0)=0$, a contradiction.

Thus if any case $(r, 2)$ arises, the underlying Breuil module must be a split extension

$$
\begin{array}{ll}
\mathcal{M}=\left(\mathbf{F}_{3}[u] /\left(u^{36}\right)\right) \mathbf{e}_{1} \oplus\left(\mathbf{F}_{3}[u] /\left(u^{36}\right)\right) \mathbf{e}_{\omega}^{\prime}, & \mathcal{M}_{1}=\left\langle u^{2} \mathbf{e}_{1}, u^{r} \mathbf{e}_{\omega}^{\prime}\right\rangle, \\
\phi_{1}\left(u^{2} \mathbf{e}_{1}\right)=\mathbf{e}_{1}, & \phi_{1}\left(u^{r} \mathbf{e}_{\omega}^{\prime}\right)=\mathbf{e}_{\omega}^{\prime}
\end{array}
$$

(so $N \circ \phi_{1}=0$ ), with

$$
\begin{gathered}
\widehat{\gamma}_{2} \mathbf{e}_{1}=\mathbf{e}_{1}, \quad \widehat{\gamma}_{2} \mathbf{e}_{\omega}^{\prime}=\mathbf{e}_{\omega}^{\prime} \\
\widehat{\gamma}_{4} \mathbf{e}_{1}=\sqrt{-1} \mathbf{e}_{1}, \quad \widehat{\gamma}_{4} \mathbf{e}_{\omega}^{\prime}=(-\sqrt{-1})^{r / 2} \mathbf{e}_{\omega}^{\prime} .
\end{gathered}
$$

We also have

$$
\widehat{\gamma_{3}^{ \pm 1}} \mathbf{e}_{1}=H_{\gamma_{3}^{ \pm 1}}(u)^{-3} \mathbf{e}_{1} \quad \widehat{\gamma_{3}^{ \pm 1}} \mathbf{e}_{\omega}^{\prime}=H_{\gamma_{3}^{ \pm 1}}(u)^{-3 r / 2} \mathbf{e}_{\omega}^{\prime}+h_{ \pm 1}(u) \mathbf{e}_{1}
$$

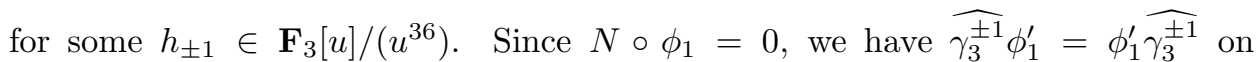
$\mathcal{M}_{1}^{\prime}$. Evaluating this on $u^{r} \mathbf{e}_{\omega}^{\prime}$ and comparing coefficients of $\mathbf{e}_{1}$ gives $h_{ \pm 1}(u)=$ $u^{3(r-2)} h_{ \pm 1}(u)^{3} H_{\gamma_{3}^{ \pm 1}}(u)^{3 r}$. This forces $h_{ \pm 1}(u)=0$ if $r \neq 2$. If $r=2$ it forces $h_{ \pm 1}(u)=c_{ \pm 1}\left(1 \pm u^{18}\right)$ for some $c_{ \pm 1} \in \mathbf{F}_{3}$. We will show $c_{-1}=c_{1}=0$. Indeed, evaluating the congruence

$$
\phi \equiv\left(\widehat{\gamma}_{3} \widehat{\gamma}_{2}-\widehat{\gamma_{3}^{-1}} \widehat{\gamma}_{2}\right) \bmod u \mathcal{M}^{\prime}
$$

on $\mathbf{e}_{\omega}^{\prime}$ gives

$$
0=\phi\left(\mathbf{e}_{\omega}^{\prime}\right) \equiv\left(c_{1}-c_{-1}\right) \mathbf{e}_{1} \bmod u \mathcal{M}^{\prime}
$$

so that $c_{-1}=c_{1}$. On the other hand the congruence

$$
\widehat{\gamma}_{3} \widehat{\gamma}_{4} \equiv \widehat{\gamma}_{4} \widehat{\gamma_{3}^{-1}} \bmod u \mathcal{M}^{\prime}
$$

gives

$$
\left(\begin{array}{cc}
1 & c_{1} \\
0 & 1
\end{array}\right)\left(\begin{array}{cc}
\sqrt{-1} & 0 \\
0 & -\sqrt{-1}
\end{array}\right)=\left(\begin{array}{cc}
\sqrt{-1} & 0 \\
0 & -\sqrt{-1}
\end{array}\right)\left(\begin{array}{cc}
1 & c_{-1} \\
0 & 1
\end{array}\right)
$$

in $M_{2}\left(\mathbf{F}_{3}\right)$, so $c_{-1}=-c_{1}$. Thus $c_{-1}=c_{1}=0$ and $h_{ \pm 1}=0$ for $r=2$ as well. Thus for $r=2,6$ and 10 the Breuil module with descent data $\mathcal{M}^{\prime}$ is split, so $\bar{\rho}$ is split, a contradiction.

This rules out the possibilities $(2,2),(6,2)$ and $(10,2)$. Using Cartier duality we can also rule out $(10,10)$ and $(10,6)$. We are left with the four possible pairs $(r, s)$ 
as asserted in the proposition and must determine which possibilities arise in each case.

Next consider the case $(r, s)=(2,6)$. Using the same analysis as in the $(r, s)=$ $(2,6)$ case in Proposition 8.2.1 we find that the possibilities for the Breuil module $\mathcal{M}$ are the ones in the statement of the proposition (and $N \circ \phi_{1}=0$ is easy to check), though we only know that

$$
\widehat{\gamma}_{4}\left(\mathbf{e}_{1}\right)=-\sqrt{-1} \mathbf{e}_{1}, \widehat{\gamma}_{4}\left(\mathbf{e}_{\omega}^{\prime}\right)=-\sqrt{-1} \mathbf{e}_{\omega}^{\prime}+h_{\gamma_{4}}(u) \mathbf{e}_{1}
$$

for some $h_{\gamma_{4}}(u) \in \mathbf{F}_{9}[u] / u^{36}$. The conditions

$$
\widehat{\gamma}_{4}\left(\mathcal{M}_{1}^{\prime}\right) \subseteq \mathcal{M}_{1}^{\prime}, \quad \widehat{\gamma}_{4} \circ \phi_{1}^{\prime}=\phi_{1}^{\prime} \circ \widehat{\gamma}_{4} \text { on } \mathcal{M}_{1}^{\prime}
$$

are equivalent to

$$
h_{\gamma_{4}} \equiv 0 \bmod u^{4}, h_{\gamma_{4}}=-\left(\frac{h_{\gamma_{4}}}{u^{4}}\right)^{3} .
$$

The solutions to this are $h_{\gamma_{4}}=a \sqrt{-1} u^{6}$ for $a \in \mathbf{F}_{3}$. Replacing $\mathbf{e}_{\omega}^{\prime}$ by $\mathbf{e}_{\omega}^{\prime}+a u^{6} \mathbf{e}_{1}$ preserves our standardized form but makes $h_{\gamma_{4}}=0$ :

$$
\widehat{\gamma}_{4}\left(\mathbf{e}_{1}\right)=-\sqrt{-1} \mathbf{e}_{1}, \quad \widehat{\gamma}_{4}\left(\mathbf{e}_{\omega}^{\prime}\right)=-\sqrt{-1} \mathbf{e}_{\omega}^{\prime} .
$$

The wild descent data must have the form

$$
\widehat{\gamma_{3}^{ \pm 1}}\left(\mathbf{e}_{1}\right)=\mathbf{e}_{1}, \widehat{\gamma_{3}^{ \pm 1}}\left(\mathbf{e}_{\omega}^{\prime}\right)=\left(1 \pm u^{18}\right) \mathbf{e}_{\omega}^{\prime}+h_{ \pm 1}(u) \mathbf{e}_{1}
$$

for some $h_{ \pm 1} \in \mathbf{F}_{9}[u] / u^{36}$. The conditions

$$
\widehat{\gamma_{3}^{ \pm 1}}\left(\mathcal{M}_{1}^{\prime}\right) \subseteq \mathcal{M}_{1}^{\prime}, \quad \widehat{\gamma_{3}^{ \pm 1}} \circ \phi_{1}^{\prime}=\phi_{1}^{\prime} \circ \widehat{\gamma_{3}^{ \pm 1}} \text { on } \mathcal{M}_{1}^{\prime}
$$

(recall $N \circ \phi_{1}=0$ ) are equivalent to

$$
h_{ \pm 1} \equiv 0 \bmod u^{4}, h_{ \pm 1}=\left(1 \pm u^{18}\right)\left(\frac{h_{ \pm 1}}{u^{4}}\right)^{3}
$$

whose solutions are

$$
h_{ \pm 1}=c_{ \pm 1} u^{6}\left(1 \pm u^{18}\right)
$$

for some $c_{ \pm 1} \in \mathbf{F}_{3}$. Since $N \circ \phi_{1}=0$, we have

$$
\widehat{\gamma_{3}^{ \pm 1}} \circ \widehat{\gamma_{3}^{\mp 1}} \circ \phi_{1}^{\prime}=\phi_{1}^{\prime} \text {, }
$$

so

$$
c_{-1}=-c_{1} .
$$

Using Lemma 5.2 .2 and Corollary 5.6 .2 we see that all of these possibilities are well defined. We also see that $\mathcal{J}$ annihilates $\mathcal{M} / u \mathcal{M} \otimes \mathbf{F}_{9}$. It is straightforward to check that generic splitting over $F^{\prime}$ (which is equivalent to generic splitting over $F$ ) is equivalent to $c=0$, and that such splitting is compatible with descent data (i.e. descends to $\mathbf{Q}_{3}$ ) if and only if $c=c_{1}=0$. For dimension reasons, the map on Ext $^{1}$ 's is therefore an isomorphism.

Now consider the case $(r, s)=(2,10)$. Here we have

$$
\mathcal{M}_{1}=\left\langle u^{10} \mathbf{e}_{1}, u^{2} \mathbf{e}_{\omega}^{\prime}+h \mathbf{e}_{1}\right\rangle
$$

for some $h \in \mathbf{F}_{3}[u] / u^{36}$, with

$$
\phi_{1}\left(u^{10} \mathbf{e}_{1}\right)=\mathbf{e}_{1}, \quad \phi_{1}\left(u^{2} \mathbf{e}_{\omega}^{\prime}+h \mathbf{e}_{1}\right)=\mathbf{e}_{\omega}^{\prime}
$$


and

$$
\widehat{\gamma}_{4}\left(\mathbf{e}_{1}\right)=\sqrt{-1} \mathbf{e}_{1}, \widehat{\gamma}_{4}\left(\mathbf{e}_{\omega}^{\prime}\right)=-\sqrt{-1} \mathbf{e}_{\omega}^{\prime}
$$

In order that

$$
\widehat{\gamma}_{4}\left(\mathcal{M}_{1}^{\prime}\right) \subseteq \mathcal{M}_{1}^{\prime}, \quad \widehat{\gamma}_{4} \circ \phi_{1}^{\prime}=\phi_{1}^{\prime} \circ \widehat{\gamma}_{4} \text { on } \mathcal{M}_{1}^{\prime},
$$

it is necessary and sufficient that

$$
h \equiv h(-\sqrt{-1} u) \bmod u^{22} .
$$

But $\left\{u^{10} t-u^{2} t^{3} \mid t \in \mathbf{F}_{3}[u] / u^{36}\right\}$ is spanned by $u^{13}-u^{11}, u^{12}-u^{8}, u^{11}-u^{5}, u^{10}-u^{2}$, and all multiples of $u^{15}$, so we may suppose

$$
h=c^{\prime \prime}+c^{\prime} u^{4}+c u^{8},
$$

for some $c^{\prime \prime}, c^{\prime}, c \in \mathbf{F}_{3}$, at the expense of possibly losing the diagonal form of $\widehat{\gamma}_{4}$.

The monodromy operator satisfies

$$
N\left(\mathbf{e}_{1}\right)=0, \quad N\left(\mathbf{e}_{\omega}^{\prime}\right)=\left(c^{\prime \prime} u^{6}-c^{\prime} u^{18}+c^{\prime \prime} u^{30}\right) \mathbf{e}_{1} .
$$

Since the wild descent data must take the form

$$
\widehat{\gamma_{3}^{ \pm 1}}\left(\mathbf{e}_{1}\right)=\left(1 \mp u^{18}\right) \mathbf{e}_{1}, \widehat{\gamma_{3}^{ \pm 1}}\left(\mathbf{e}_{\omega}^{\prime}\right)=\left(1 \pm u^{18}\right) \mathbf{e}_{\omega}^{\prime}+h_{ \pm 1} \mathbf{e}_{1}
$$

for some $h_{ \pm 1} \in \mathbf{F}_{9}[u] / u^{36}$, we compute

$$
\widehat{\gamma_{3}^{ \pm 1}}\left(u^{2} \mathbf{e}_{\omega}^{\prime}+h \mathbf{e}_{1}\right)=H_{\gamma_{3}^{ \pm 1}}^{2}(u)\left(1 \pm u^{18}\right)\left(u^{2} \mathbf{e}_{\omega}^{\prime}+h \mathbf{e}_{1}\right)+f_{ \pm 1}(u) \mathbf{e}_{1},
$$

where

$$
f_{ \pm 1}(u)=-h H_{\gamma_{3}^{ \pm 1}}^{2}(u)\left(1 \pm u^{18}\right)+u^{2} H_{\gamma_{3}^{ \pm 1}}^{2} h_{ \pm 1}+\left(1 \mp u^{18}\right) h\left(u H_{\gamma_{3}^{ \pm 1}}\right) .
$$

Thus, in order that $\widehat{\gamma_{3}^{ \pm 1}}\left(\mathcal{M}_{1}^{\prime}\right) \subseteq \mathcal{M}_{1}^{\prime}$, it is necessary and sufficient that $f_{ \pm 1}$ satisfies

$$
f_{ \pm 1} \equiv 0 \bmod u^{10} \text {. }
$$

Using (9.2.1) and $H_{\gamma_{3}^{ \pm 1}} \equiv 1 \mp u^{6} \bmod 3$, this amounts to

$$
h_{ \pm 1} \equiv \pm c^{\prime \prime} u^{4} \bmod u^{8}
$$

However, $N \circ \phi_{1}\left(\mathcal{M}_{1}\right) \subseteq u^{6} \mathcal{M}$, so

$$
\widehat{\gamma_{3}^{ \pm 1}} \circ \phi_{1}^{\prime} \equiv \phi_{1}^{\prime} \circ \widehat{\gamma_{3}^{ \pm 1}} \bmod u^{6} \mathcal{M}^{\prime}
$$

when evaluated on $\mathcal{M}_{1}^{\prime}$. This gives

$$
h_{ \pm 1} \equiv\left(\frac{f_{ \pm 1}}{u^{10}}\right)^{3} \bmod u^{6}
$$

Since $h_{ \pm 1}$ is a cube modulo $u^{6}$, by (9.2.3) we must have $c^{\prime \prime}=0$, and so $N \circ \phi_{1} \equiv$ $0 \bmod u^{18} \mathcal{M}$. Thus, $\widehat{\gamma_{3}^{ \pm 1}}$ and $\phi_{1}^{\prime}$ commute modulo $u^{18} \mathcal{M}^{\prime}$ when evaluated on $\mathcal{M}_{1}^{\prime}$, so we get

$$
h_{ \pm 1} \equiv\left(\frac{f_{ \pm 1}}{u^{10}}\right)^{3} \bmod u^{18}
$$

and $h_{ \pm 1}$ is a cube modulo $u^{18}$.

On the other hand, with $c^{\prime \prime}=0$, we see from (9.2.3) that

$$
h_{ \pm 1} \equiv 0 \bmod u^{8} \text {. }
$$


Because $h_{ \pm 1}$ is a cube modulo $u^{18}$, we get the slight improvement

$$
h_{ \pm 1} \equiv 0 \bmod u^{9} \text {. }
$$

Combining this with the vanishing of $c^{\prime \prime}$, we deduce from (9.2.2) that $f_{ \pm 1} \equiv$ $\pm c^{\prime} u^{10} \bmod u^{11}$, so by 9.2.4

$$
h_{ \pm 1} \equiv \pm c^{\prime} \bmod u \text {. }
$$

This forces $c^{\prime}=0$, so $N \circ \phi_{1}=0$. Thus, $\widehat{\gamma_{3}^{ \pm 1}}$ and $\phi_{1}^{\prime}$ commute on $\mathcal{M}_{1}^{\prime}$, so

$$
h_{ \pm 1}=\left(\frac{f_{ \pm 1}}{u^{10}}\right)^{3}
$$

in $\mathbf{F}_{9}[u] / u^{36}$. Using $h=c u^{8}$ this becomes (via (9.2.2) )

SO

$$
h_{ \pm 1}=\left(1 \pm u^{18}\right)\left(\frac{h_{ \pm 1}}{u^{8}}\right)^{3},
$$

$$
h_{ \pm 1}=c_{ \pm 1} u^{12}\left(1 \pm u^{18}\right)
$$

for some $c_{ \pm 1} \in \mathbf{F}_{3}$. As before, we get $c_{-1}=-c_{1}$.

Now we "diagonalise" $\widehat{\gamma}_{4}$. Since we have

$$
\widehat{\gamma}_{4}\left(\mathbf{e}_{1}\right)=\sqrt{-1} \mathbf{e}_{1}, \quad \widehat{\gamma}_{4}\left(\mathbf{e}_{\omega}^{\prime}\right)=-\sqrt{-1} \mathbf{e}_{\omega}^{\prime}+h_{\gamma_{4}}(u) \mathbf{e}_{1}
$$

for some $h_{\gamma_{4}} \in \mathbf{F}_{9}[u] / u^{36}$, the conditions

$$
\widehat{\gamma}_{4}\left(\mathcal{M}_{1}^{\prime}\right) \subseteq \mathcal{M}_{1}^{\prime}, \quad \widehat{\gamma}_{4} \circ \phi_{1}^{\prime}=\phi_{1}^{\prime} \circ \widehat{\gamma}_{4} \text { on } \mathcal{M}_{1}^{\prime}
$$

are equivalent to

$$
h_{\gamma_{4}} \equiv 0 \bmod u^{8}, \quad h_{\gamma_{4}}=-\left(\frac{h_{\gamma_{4}}}{u^{8}}\right)^{3}
$$

which is to say

$$
h_{\gamma_{4}}=a \sqrt{-1} u^{12}
$$

for some $a \in \mathbf{F}_{3}$. Replacing $\mathbf{e}_{\omega}^{\prime}$ by $\mathbf{e}_{\omega}^{\prime}+a u^{12} \mathbf{e}_{1}$ then puts us in a setting with $a=0$. Thus all extensions have the form asserted in the proposition. It is easy to check that in each case $\mathcal{J}$ annihilates $(\mathcal{M} / u \mathcal{N}) \otimes \mathbf{F}_{9}$.

Pushout by the non-zero map $\mathcal{M}_{6,1}^{\prime} \rightarrow \mathcal{M}_{10,1}^{\prime}$ in $\underline{\phi 1}_{1} D D_{F^{\prime} / \mathbf{Q}_{3}}$ induced by $\mathbf{e} \mapsto u^{6} \mathbf{e}$ takes our $(2,6)$ examples to our $(2,10)$ examples (compatibly with the labelling of parameters $c, c_{1}$ as in the statement of the proposition). Thus all 9 possibilities for $\left(c, c_{1}\right)$ do occur and we get an isomorphism of Ext ${ }^{1}$ 's as asserted. Moreover, generic splitting over $F^{\prime}$ (which is equivalent to generic splitting over $F$ ) is equivalent to $c=0$, and such splitting is compatible with descent data (i.e. descends to $\mathbf{Q}_{3}$ ) if and only if $c=c_{1}=0$.

Using Cartier duality and the case $(r, s)=(2,6)$, we see that in the case $(r, s)=$ $(6,10)$ the map of Ext $^{1}$ 's is an isomorphism. It is easy to check that the objects in our asserted list of 9 possibilities for $(r, s)=(6,10)$ are well defined and that pullback by the non-zero map $\mathcal{M}_{2, \omega}^{\prime} \rightarrow \mathcal{M}_{6, \omega}^{\prime}$ induced by $\mathbf{e} \mapsto u^{6} \mathbf{e}$ takes these to our $(2,10)$ examples (compatibly with the labelling of parameters $\left.c, c_{1}\right)$.

Finally, we turn to the case $(r, s)=(6,6)$. Choosing a basis with respect to which $\widehat{\gamma}_{4}$ has a diagonal action, the conditions

$$
\widehat{\gamma}_{4}\left(\mathcal{M}_{1}^{\prime}\right) \subseteq \mathcal{M}_{1}^{\prime}, \quad \widehat{\gamma}_{4} \circ \phi_{1}^{\prime}=\phi_{1}^{\prime} \circ \widehat{\gamma}_{4} \text { on } \mathcal{M}_{1}^{\prime}
$$


are equivalent to

$$
h(u) \equiv h(-\sqrt{-1} u) \bmod u^{18} .
$$

Since $\left\{u^{6} t-u^{6} t^{3} \mid t \in \mathbf{F}_{3}[u] / u^{36}\right\}$ consists of multiples of $u^{7}$, we may change $\mathbf{e}_{\omega}^{\prime}$ to get

$$
h=c+c^{\prime} u^{4}
$$

for some $c, c^{\prime} \in \mathbf{F}_{3}$, with

$$
\widehat{\gamma}_{4}\left(\mathbf{e}_{1}\right)=-\sqrt{-1} \mathbf{e}_{1}, \quad \widehat{\gamma}_{4}\left(\mathbf{e}_{\omega}^{\prime}\right)=\sqrt{-1} \mathbf{e}_{\omega}^{\prime}+h_{\gamma_{4}}(u) \mathbf{e}_{1}
$$

for some $h_{\gamma_{4}} \in \mathbf{F}_{9}[u] / u^{36}$. Feeding this into (9.2.5) we get $h_{\gamma_{4}}=-h_{\gamma_{4}}^{3}$, so $h_{\gamma_{4}}=$ $\sqrt{-1} a$ for some $a \in \mathbf{F}_{3}$. Replacing $\mathbf{e}_{\omega}^{\prime}$ by $\mathbf{e}_{\omega}^{\prime}-a \mathbf{e}_{1}$ returns us to the setting with "diagonal" $\widehat{\gamma}_{4}$-action and preserves the standardizations we have made so far.

It is easy to compute $N\left(\mathbf{e}_{\omega}^{\prime}\right)=c^{\prime} u^{30} \mathbf{e}_{1}$ (and we know $N\left(\mathbf{e}_{1}\right)=0$ ). The "wild" descent data is

$$
\widehat{\gamma_{3}^{ \pm 1}}\left(\mathbf{e}_{1}\right)=\mathbf{e}_{1}, \widehat{\gamma_{3}^{ \pm 1}}\left(\mathbf{e}_{\omega}^{\prime}\right)=\mathbf{e}_{\omega}^{\prime}+h_{ \pm 1} \mathbf{e}_{1}
$$

for some $h_{ \pm 1} \in \mathbf{F}_{9}[u] / u^{36}$. Using the congruence for $t_{\gamma_{3}^{ \pm 1}}$ in $\oint 6.5$, the identity

$$
\widehat{\gamma_{3}^{ \pm 1}} \circ \phi_{1}^{\prime}=\left(1+t_{\gamma_{3}^{ \pm 1}} \cdot N\right) \circ \phi_{1}^{\prime} \circ \widehat{\gamma_{3}^{ \pm 1}}
$$

on $\mathcal{M}_{1}^{\prime}$ amounts to the condition

$$
h_{ \pm 1}=h_{ \pm 1}^{3} \mp c^{\prime} u^{12}-c^{\prime} u^{30}
$$

whose solutions are

$$
h_{ \pm 1}=c_{ \pm 1} \mp c^{\prime} u^{12}-c^{\prime} u^{30}
$$

for some $c_{ \pm 1} \in \mathbf{F}_{3}$. The identity

$$
\widehat{\gamma_{3}^{ \pm 1}} \circ \widehat{\gamma_{3}^{\mp 1}} \equiv 1 \bmod u \mathcal{M}^{\prime}
$$

implies $c_{-1}=-c_{1}$. Thus

$-\mathbf{V}_{\mathcal{M}^{\prime}}\left(\mathbf{e}_{\omega}^{\prime}\right) \equiv \phi\left(\mathbf{e}_{\omega}^{\prime}\right) \equiv-c \mathbf{e}_{1} \bmod u \mathcal{M}^{\prime}, \quad\left(\widehat{\gamma}_{3} \circ \widehat{\gamma}_{2}-\widehat{\gamma_{3}^{-1}} \widehat{\gamma}_{2}\right)\left(\mathbf{e}_{\omega}^{\prime}\right) \equiv-c_{1} \mathbf{e}_{1} \bmod u \mathcal{M}^{\prime}$

Thus $\mathcal{J}$ annihilates $(\mathcal{M} / u \mathcal{M}) \otimes \mathbf{F}_{3} \mathbf{F}_{9}$ if and only if $c_{1}=c$.

By Lemma 5.2.2 and Corollary 5.6.2, it is easy to see that all of these objects are well defined. The kernel of

$$
\operatorname{Ext}_{{\underline{\phi_{1} D D}}_{F^{\prime} / \mathbf{Q}_{3}, \mathcal{J}}^{1}}\left(\mathcal{M}_{6, \omega}^{\prime}, \mathcal{M}_{6, \mathbf{1}}^{\prime}\right) \longrightarrow \operatorname{Ext}_{\mathbf{F}_{3}\left[G_{F}\right]}^{1}(1, \omega)
$$

consists of pairs $(c,-c)$, where generic splittings are induced by any of the (nonzero) Breuil module maps

$$
\mathcal{M}(0,1) \longrightarrow \mathcal{M}
$$

defined by

$$
\mathbf{e} \longmapsto u^{9} \mathbf{e}_{\omega}^{\prime}+\left(\tilde{c} u^{9}+c u^{3}\right) \mathbf{e}_{1}=u\left(c+u^{2} \tilde{c}\right) u^{6} \mathbf{e}_{1}+u^{3}\left(u^{6} \mathbf{e}_{\omega}^{\prime}+\left(c-c u^{4}\right) \mathbf{e}_{1}\right)
$$

with $\tilde{c} \in \mathbf{F}_{3}$. Thus, the pairs $\left(c, c^{\prime}\right)$ corresponding to the $\bar{\rho}$ which are split over $F$ (or equivalently, split over $F^{\prime}$ ) are exactly those for which $c+c^{\prime}=0$. The map

$$
\operatorname{Ext}_{{\underline{\phi_{1} D D}}_{F^{\prime} / \mathbf{Q}_{3}, \mathcal{J}}^{1}}^{1}\left(\mathcal{M}_{6, \omega}^{\prime}, \mathcal{M}_{6, \mathbf{1}}^{\prime}\right) \longrightarrow \operatorname{Ext}_{\mathbf{F}_{3}\left[G_{3}\right]}^{1}(1, \omega)
$$

is therefore injective, because the splitting given above respects descent data if and only if $\tilde{c}=c_{1}=0$. 
It remains to establish which of the given extensions of Breuil modules correspond to peu ramifié extensions of $\mathbf{Z} / 3 \mathbf{Z}$ by $\mu_{3}$ over $\mathbf{Q}_{3}$. We noted above that the maps among the $\operatorname{Ext}_{{\underline{\phi_{1} D D}}_{F^{\prime} / \mathbf{Q}_{3}}^{1}}$ 's in the $(2,6),(6,10),(2,10)$ cases induced by pushout/pullback along $\mathbf{e} \mapsto u^{6} \mathbf{e}$ are compatible with the parametrisation by pairs $\left(c, c_{1}\right)$. With a little more care, one checks that the maps

$$
\begin{aligned}
& \operatorname{Ext}_{\underline{\phi_{1} D D}}^{1} F_{F^{\prime} / \mathbf{Q}_{3}, J}\left(\mathcal{M}_{2, \omega}^{\prime}, \mathcal{M}_{6, \mathbf{1}}^{\prime}\right) \longleftarrow \operatorname{Ext}_{\underline{\phi_{1} D D}}^{1} F_{F^{\prime} / \mathbf{Q}_{3}, J}\left(\mathcal{M}_{6, \omega}^{\prime}, \mathcal{M}_{6, \mathbf{1}}^{\prime}\right) \\
& \longrightarrow \operatorname{Ext}_{\underline{\phi 1}_{1} D D_{F^{\prime} / \mathbf{Q}_{3}, j}}^{1}\left(\mathcal{M}_{6, \omega}^{\prime}, \mathcal{M}_{10, \mathbf{1}}^{\prime}\right)
\end{aligned}
$$

induced by $\mathbf{e} \mapsto u^{6} \mathbf{e}$ send the pair $\left(c, c^{\prime}\right)$ in the middle to the pair $\left(c+c^{\prime}, c\right)$ on either end (to construct the necessary commutative diagrams of short exact sequences in the two cases, use the maps

$$
\left(\mathbf{e}_{\omega}^{\prime}, \mathbf{e}_{1}\right) \longmapsto\left(u^{6} \mathbf{e}_{\omega}^{\prime}-c^{\prime} \mathbf{e}_{1}, \mathbf{e}_{1}\right),\left(\mathbf{e}_{\omega}^{\prime}, \mathbf{e}_{1}\right) \longmapsto\left(\mathbf{e}_{\omega}^{\prime}+c^{\prime} \mathbf{e}_{1}, u^{6} \mathbf{e}_{1}\right)
$$

respectively). This reduces us to checking the $(6,6)$ case.

By Corollary 2.3.2, the two très ramifié extensions, $\bar{\rho}_{1}$ and $\bar{\rho}_{2}$, of 1 by $\omega$ which are non-split over $F$ arise from elliptic curves, $E_{1}$ and $E_{2}$, over $\mathbf{Q}_{3}$ for which $\rho_{E_{j}, 3}$ is potentially Barsotti-Tate with extended type $\tau_{i}^{\prime}$ (see 6.5). Let $\mathcal{G}_{j}$ denote the 3 -torsion in the Néron model of $E_{j}$ over $\mathcal{O}_{F}$. From the universal property of Néron models we see that $\mathcal{G}_{j}^{\prime}=\mathcal{G}_{j} \times_{\mathcal{O}_{F}} \mathcal{O}_{F^{\prime}}$ inherits descent data $\{[g]\}$ over $\mathbf{Q}_{3}$. By the same argument used at the end of 44.6] we see that $\left(\mathcal{G}_{j}^{\prime},\{[g]\}\right)$ is an object of

$\underline{\phi}_{1} D D_{F^{\prime} / \mathbf{Q}_{3}, \mathcal{J}}$. Moreover we see that $\mathbf{F} \neq 0$ on $D\left(\mathcal{G}_{j}\right)$. Since all non- $(6,6)$ cases above have $\phi \equiv 0 \bmod u \mathcal{M}$, by the parts of Proposition 9.2.1 which we have already proved we see that $\mathcal{M}_{\pi}\left(\mathcal{G}_{j}^{\prime},\{[g]\}\right)$ is an extension of $\mathcal{M}_{6, \omega}^{\prime}$ by $\mathcal{M}_{6,1}^{\prime}$ and correspond to a pair $\left(c, c^{\prime}\right.$ ) with $c \neq 0$ (since $\mathbf{F} \neq 0$ ) and $c+c^{\prime} \neq 0$ (by our analysis of (9.2.6), since $\bar{\rho}_{i}$ is non-split over $\left.F\right)$. Hence $\mathcal{M}_{\pi}\left(\mathcal{G}_{1}^{\prime},\{[g]\}\right)$ and $\mathcal{M}_{\pi}\left(\mathcal{G}_{2}^{\prime},\{[g]\}\right)$ must correspond in some order to the lines $c^{\prime}=0$ and $c=c^{\prime}$ in $\mathbf{F}_{3}^{2}$.

As a non-split peu ramifié extension of 1 by $\omega$ remains non-split over $F^{\prime}$, we see that the peu ramifié line in

$$
\operatorname{Ext}_{{\underline{\phi_{1} D D}}_{F^{\prime} / \mathbf{Q}_{3}, \mathfrak{j}}^{1}}^{1}\left(\mathcal{M}_{6, \omega}^{\prime}, \mathcal{M}_{6, \mathbf{1}}^{\prime}\right) \cong \operatorname{Ext}_{\mathbf{F}_{3}\left[G_{3}\right]}^{1}(1, \omega)
$$

cannot correspond to $c+c^{\prime}=0$. By the above analysis it cannot correspond to $c^{\prime}=0$ or $c-c^{\prime}=0$. Thus it must correspond to the remaining line $c=0$.

The properties of $\phi$ in the cases listed in Proposition 9.2.1 make it clear that the $(6,6)$ case there is "different". We will see further manifestations of this difference later.

\subsection{Further rank two calculations.}

Lemma 9.3.1. For $(r, s)=(2,6),(6,10)$ and $(2,10)$ we have

$$
\operatorname{Ext}_{\underline{\phi_{1} D D_{F^{\prime} / \mathbf{Q}_{3}, j}}}^{1}\left(\mathcal{M}_{s, 1}^{\prime}, \mathcal{M}_{r, \omega}^{\prime}\right)=(0) \text {. }
$$

Proof. The $(6,10)$ case follows from the $(2,6)$ case by Cartier duality. Thus, we assume $r=2, s \in\{6,10\}$. Let $\left(\mathcal{M}^{\prime},\{\widehat{g}\}\right)$ be such an extension. By Lemma 8.1.2 $\left(\mathcal{M}^{\prime},\{\widehat{g}\}\right)$ arises from a Breuil module over $\mathcal{O}_{F}$ of the form

$$
\mathcal{M}=\left(\mathbf{F}_{3}[u] / u^{36}\right) \mathbf{e}_{\omega} \oplus\left(\mathbf{F}_{3}[u] / u^{36}\right) \mathbf{e}_{1}^{\prime}, \quad \mathcal{M}_{1}=\left\langle u^{2} \mathbf{e}_{\omega}, u^{s} \mathbf{e}_{1}^{\prime}+h \mathbf{e}_{\omega}\right\rangle
$$

with

$$
\phi_{1}\left(u^{2} \mathbf{e}_{\omega}\right)=\mathbf{e}_{\omega}, \quad \phi_{1}\left(u^{s} \mathbf{e}_{1}^{\prime}+h \mathbf{e}_{\omega}\right)=\mathbf{e}_{1}^{\prime}
$$


and

$$
\widehat{\gamma}_{4}\left(\mathbf{e}_{\omega}\right)=-\sqrt{-1} \mathbf{e}_{\omega}, \quad \widehat{\gamma}_{4}\left(\mathbf{e}_{1}^{\prime}\right)=-(-\sqrt{-1})^{s / 2} \mathbf{e}_{1}^{\prime},
$$

where $h \in \mathbf{F}_{3}[u] / u^{36}$.

The combined conditions

$$
\widehat{\gamma}_{4}\left(\mathcal{M}_{1}^{\prime}\right) \subseteq \mathcal{M}_{1}^{\prime}, \widehat{\gamma}_{4} \circ \phi_{1}^{\prime}=\phi_{1}^{\prime} \circ \widehat{\gamma}_{4} \text { on } \mathcal{M}_{1}^{\prime}
$$

are equivalent to

$$
(\sqrt{-1})^{s / 2} h(u) \equiv(\sqrt{-1}) h(-\sqrt{-1} u) \bmod u^{14}
$$

Treating the cases $s=6$ and $s=10$ separately, we conclude from Lemma 5.2 .2 that we may change $\mathbf{e}_{1}^{\prime}$ so that $h=0$ when $s=6$ and $h \in \mathbf{F}_{3}$ when $s=10$. As a result of this change, we only have

$$
\widehat{\gamma}_{4}\left(\mathbf{e}_{\omega}\right)=-\sqrt{-1} \mathbf{e}_{\omega}, \quad \widehat{\gamma}_{4}\left(\mathbf{e}_{1}^{\prime}\right)=-(-\sqrt{-1})^{s / 2} \mathbf{e}_{1}^{\prime}+h_{\gamma_{4}}(u) \mathbf{e}_{\omega} .
$$

However, with $h \in \mathbf{F}_{3}$ when $s=10$ and $h=0$ when $s=6$, the condition

$$
\widehat{\gamma}_{4} \circ \phi_{1}^{\prime}=\phi_{1}^{\prime} \circ \widehat{\gamma}_{4} \text { on } \mathcal{M}_{1}^{\prime}
$$

forces $h_{\gamma_{4}}=-u^{3(s-2)} h_{\gamma_{4}}^{3}$, so that in fact $h_{\gamma_{4}}=0$ after all.

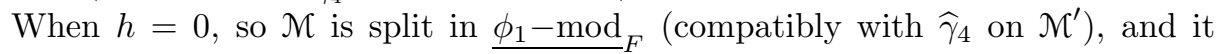
is easy to check (using $N=0$ ) that the "wild" descent data $\widehat{\gamma_{3}^{ \pm 1}}$ must also be diagonal, so we have the desired splitting in $\underline{\phi}_{1} D D F^{\prime} / \mathbf{Q}_{3}$.

It remains to consider the case $(r, s)=(2,10)$ with $h=c \in \mathbf{F}_{3}$. It is easy to compute

$$
N\left(\mathbf{e}_{\omega}\right)=0, \quad N\left(\mathbf{e}_{1}^{\prime}\right)=-c u^{30} \mathbf{e}_{\omega} .
$$

The wild descent data must have the form

$$
\widehat{\gamma_{3}^{ \pm 1}}\left(\mathbf{e}_{\omega}\right)=\left(1 \pm u^{18}\right) \mathbf{e}_{\omega}, \widehat{\gamma_{3}^{ \pm 1}}\left(\mathbf{e}_{1}^{\prime}\right)=\left(1 \mp u^{18}\right) \mathbf{e}_{1}^{\prime}+h_{ \pm 1} \mathbf{e}_{\omega}
$$

with $h_{ \pm 1} \in \mathbf{F}_{9}[u] / u^{36}$.

It is straightforward to check that $\widehat{\gamma_{3}^{ \pm 1}}\left(\mathcal{M}_{1}^{\prime}\right) \subseteq \mathcal{M}_{1}^{\prime}$, and then the condition

$$
\widehat{\gamma_{3}^{ \pm 1}} \circ \phi_{1}^{\prime}=\left(1+t_{\gamma_{3}^{ \pm 1}} \cdot N\right) \circ \phi_{1}^{\prime} \circ \widehat{\gamma_{3}^{ \pm 1}}
$$

on $\mathcal{M}_{1}^{\prime}$ gives

$$
h_{ \pm 1}= \pm c u^{12}+h_{ \pm 1}^{3} u^{24}\left(1 \mp u^{18}\right)+c u^{30} .
$$

The unique solution to this is

$$
h_{ \pm 1}=c\left( \pm u^{12}+u^{30}\right)
$$

Thus $\widehat{\gamma}_{3} \widehat{\gamma}_{2}-\widehat{\gamma_{3}^{-1}} \widehat{\gamma}_{2} \equiv 0 \bmod u$, while $\phi\left(\mathbf{e}_{1}^{\prime}\right) \equiv-c \mathbf{e}_{\omega} \bmod u$. This forces $c=0$. With $c=0$ we obviously have only the split extension class.

Lemma 9.3.2. The natural map

$$
\operatorname{Ext}_{\underline{\phi_{1} D D_{F^{\prime}} / \mathbf{Q}_{3}, j}}^{1}\left(\mathcal{M}_{6, \mathbf{1}}^{\prime}, \mathcal{M}_{6, \omega}^{\prime}\right) \longrightarrow \operatorname{Ext}_{\mathbf{F}_{3}\left[G_{3}\right]}^{1}(\omega, 1)
$$

is an isomorphism, with elements parametrised by pairs $\left(c, c^{\prime}\right) \in \mathbf{F}_{3}^{2}$ corresponding to

$$
\mathcal{M}=\left(\mathbf{F}_{3}[u] / u^{36}\right) \mathbf{e}_{\omega} \oplus\left(\mathbf{F}_{3}[u] / u^{36}\right) \mathbf{e}_{1}^{\prime}, \quad \mathcal{M}_{1}=\left\langle u^{6} \mathbf{e}_{\omega}, u^{6} \mathbf{e}_{1}^{\prime}+\left(c+c^{\prime} u^{4}\right) \mathbf{e}_{\omega}\right\rangle
$$


where

$$
\phi_{1}\left(u^{6} \mathbf{e}_{\omega}\right)=\mathbf{e}_{\omega}, \quad \phi_{1}\left(u^{6} \mathbf{e}_{1}^{\prime}+\left(c+c^{\prime} u^{4}\right) \mathbf{e}_{\omega}\right)=\mathbf{e}_{1}^{\prime}, \quad N\left(\mathbf{e}_{\omega}\right)=0, \quad N\left(\mathbf{e}_{1}^{\prime}\right)=c^{\prime} u^{30} \mathbf{e}_{\omega}
$$

and the descent data is

$$
\begin{gathered}
\widehat{\gamma}_{4}\left(\mathbf{e}_{\omega}\right)=\sqrt{-1} \mathbf{e}_{\omega}, \widehat{\gamma}_{4}\left(\mathbf{e}_{1}^{\prime}\right)=-\sqrt{-1} \mathbf{e}_{1}^{\prime}, \\
\widehat{\gamma_{3}^{ \pm 1}}\left(\mathbf{e}_{\omega}\right)=\mathbf{e}_{\omega}, \widehat{\gamma_{3}^{ \pm 1}}\left(\mathbf{e}_{1}^{\prime}\right)=\mathbf{e}_{1}^{\prime}+\left( \pm c \mp c^{\prime} u^{12}-c^{\prime} u^{30}\right) \mathbf{e}_{\omega} .
\end{gathered}
$$

Proof. The proof is identical to the proof of the case $(r, s)=(6,6)$ in Proposition 9.2.1 except $\sqrt{-1}$ is everywhere replaced by $-\sqrt{-1}$ and when we study splitting we give $\mathcal{M}(0,1)$ the descent data for the trivial mod 3 character (which amounts to using $\widehat{\gamma}_{4}(\mathbf{e})=-\mathbf{e}$ rather than $\left.\widehat{\gamma}_{4}(\mathbf{e})=\mathbf{e}\right)$.

Lemma 9.3.3. For $r \in\{2,10\}$, the maps

$$
\operatorname{Ext}_{\underline{\phi_{1} D D}}^{1}{ }_{F^{\prime} / \mathbf{Q}_{3}}\left(\mathcal{M}_{r, \omega}^{\prime}, \mathcal{M}_{r, \omega}^{\prime}\right) \longrightarrow \operatorname{Ext}_{\mathbf{F}_{3}\left[G_{3}\right]}^{1}(\omega, \omega)
$$

and

$$
\operatorname{Ext}_{\underline{\phi_{1} D D}}^{1}{ }_{F^{\prime} / \mathbf{Q}_{3}}\left(\mathcal{M}_{r, \mathbf{1}}^{\prime}, \mathcal{M}_{r, \mathbf{1}}^{\prime}\right) \longrightarrow \operatorname{Ext}_{\mathbf{F}_{3}\left[G_{3}\right]}^{1}(1,1)
$$

are injective and have image consisting of the 1-dimensional space of classes which split over an unramified extension of $\mathbf{Q}_{3}$.

Proof. The cases $r=10$ follow from the cases $r=2$ using Cartier duality. Thus we suppose $r=2$. We treat only the case of $\mathcal{M}_{2, \omega}^{\prime}$, the case $\mathcal{M}_{2,1}^{\prime}$ being exactly the same except that $-\sqrt{-1}$ replaces $\sqrt{-1}$ everywhere.

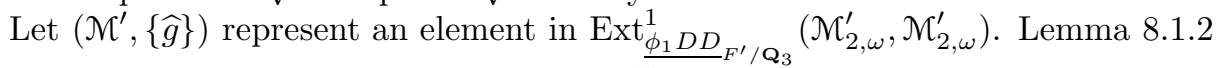
ensures the existence of an ordered $\mathbf{F}_{3}[u] / u^{36}$-basis $\mathbf{e}_{\omega}, \mathbf{e}_{\omega}^{\prime}$ of $\mathcal{M}$ such that

$$
\mathcal{M}_{1}=\left\langle u^{2} \mathbf{e}_{\omega}, u^{2} \mathbf{e}_{\omega}^{\prime}+h \mathbf{e}_{\omega}\right\rangle, \quad \phi_{1}\left(u^{2} \mathbf{e}_{\omega}\right)=\mathbf{e}_{\omega}, \quad \phi_{1}\left(u^{2} \mathbf{e}_{\omega}^{\prime}+h \mathbf{e}_{\omega}\right)=\mathbf{e}_{\omega}^{\prime}
$$

with

$$
\widehat{\gamma}_{4}\left(\mathbf{e}_{\omega}\right)=-\sqrt{-1} \mathbf{e}_{\omega}, \widehat{\gamma}_{4}\left(\mathbf{e}_{\omega}^{\prime}\right)=-\sqrt{-1} \mathbf{e}_{\omega}^{\prime} .
$$

Carrying out the usual calculation,

$$
\widehat{\gamma}_{4}\left(\mathcal{M}_{1}^{\prime}\right) \subseteq \mathcal{M}_{1}^{\prime}, \quad \phi_{1}^{\prime} \circ \widehat{\gamma}_{4}=\widehat{\gamma}_{4} \circ \phi_{1}^{\prime} \text { on } \mathcal{M}_{1}^{\prime}
$$

if and only if

$$
h \equiv-h(-\sqrt{-1} u) \bmod u^{14} .
$$

Combining this with Lemma 5.2 .2 , we may change $\mathbf{e}_{\omega}^{\prime}$ so that $h=c u^{2}$, with $c \in \mathbf{F}_{3}$, at the expense of possibly losing the diagonal form of $\widehat{\gamma}_{4}$. But with $h=c u^{2}$ and $\widehat{\gamma}_{4}\left(\mathbf{e}_{\omega}^{\prime}\right)=-\sqrt{-1} \mathbf{e}_{\omega}^{\prime}+h_{\gamma_{4}}(u) \mathbf{e}_{\omega}$, the conditions (9.3.1) imply $h_{\gamma_{4}}=-h_{\gamma_{4}}^{3}$, and so $h_{\gamma_{4}}(u)=(\sqrt{-1}) a$ for some $a \in \mathbf{F}_{3}$. Then $\widehat{\gamma}_{4}^{4}=1$ forces $a=0$, so $\widehat{\gamma}_{4}$ still has diagonal form.

It is easy to check that $N\left(\mathbf{e}_{\omega}^{\prime}\right)=0$, so $N \circ \phi_{1}=0$. Thus, we must have

$$
\widehat{\gamma_{3}^{ \pm 1}} \circ \phi_{1}^{\prime}=\phi_{1}^{\prime} \circ \widehat{\gamma_{3}^{ \pm 1}}
$$

on $\mathcal{M}_{1}^{\prime}$. Since the wild descent data has to be of the form

$$
\widehat{\gamma_{3}^{ \pm 1}}\left(\mathbf{e}_{\omega}\right)=\left(1 \pm u^{18}\right) \mathbf{e}_{\omega}, \widehat{\gamma_{3}^{ \pm 1}}\left(\mathbf{e}_{\omega}^{\prime}\right)=\left(1 \pm u^{18}\right) \mathbf{e}_{\omega}^{\prime}+h_{ \pm 1} \mathbf{e}_{\omega}
$$


for some $h_{ \pm 1} \in \mathbf{F}_{3}[u] / u^{36}$, evaluation of (19.3.2) on $u^{2} \mathbf{e}_{\omega}^{\prime}+c u^{2} \mathbf{e}_{\omega} \in \mathcal{M}_{1}$ gives $h_{ \pm 1}=$ $\left(1 \pm u^{18}\right) h_{ \pm 1}^{3}$, so $h_{ \pm 1}=c_{ \pm 1}\left(1 \pm u^{18}\right)$ for some $c_{ \pm 1} \in \mathbf{F}_{3}$. The relation $\widehat{\gamma_{3}^{ \pm 1}} \circ \widehat{\gamma}_{4} \circ$ $\widehat{\gamma_{3}^{ \pm 1}} \widehat{\gamma_{4}^{3}}\left(\mathbf{e}_{\omega}^{\prime}\right)=\mathbf{e}_{\omega}^{\prime}$ forces $c_{ \pm 1}=0$.

We now have described all possibilities in terms of the single parameter $c \in \mathbf{F}_{3}$, and it is straightforward to use Corollary 5.6 .2 to check that all of these examples are in fact well defined. Generic splittings over unramified extensions of $\mathbf{Q}_{3}$ correspond to the maps

$$
\overline{\mathbf{F}}_{3} \otimes_{\mathbf{F}_{3}} \mathcal{M}(0,1) \longrightarrow \overline{\mathbf{F}}_{3} \otimes_{\mathbf{F}_{3}} \mathcal{M}
$$

given by

$$
\mathbf{e} \longmapsto a u^{3} \mathbf{e}_{\omega}+u\left(u^{2} \mathbf{e}_{\omega}^{\prime}+c u^{2} \mathbf{e}_{\omega}\right)
$$

where $a \in \overline{\mathbf{F}}_{3}$ satisfies $a^{3}=a+c$. Such generic splittings can be defined over $\mathbf{Q}_{3}$ (i.e. without extending the residue field) if and only if $c=0$.

Lemma 9.3.4. (1) The map of groups

$$
\operatorname{Ext}_{\underline{\phi_{1} D D}}^{1}{ }_{F^{\prime} / \mathbf{Q}_{3}}\left(\mathcal{M}_{6, \mathbf{1}}^{\prime}, \mathcal{M}_{6, \mathbf{1}}^{\prime}\right) \longrightarrow \operatorname{Ext}_{\mathbf{F}_{3}\left[G_{3}\right]}^{1}(1,1)
$$

is an isomorphism.

Explicitly, the group $\operatorname{Ext}_{\underline{\phi_{1} D D_{F^{\prime} / \mathbf{Q}_{3}}}}^{1}\left(\mathcal{M}_{6, \mathbf{1}}^{\prime}, \mathcal{M}_{6, \mathbf{1}}^{\prime}\right)$ is parametrised by pairs $\left(c, c^{\prime}\right) \in \mathbf{F}_{3}^{2}$ corresponding to

$$
\mathcal{M}=\left(\mathbf{F}_{3}[u] / u^{36}\right) \mathbf{e}_{1} \oplus\left(\mathbf{F}_{3}[u] / u^{36}\right) \mathbf{e}_{1}^{\prime}, \quad \mathcal{M}_{1}=\left\langle u^{6} \mathbf{e}_{1}, u^{6} \mathbf{e}_{1}^{\prime}+\left(c u^{2}+c^{\prime} u^{6}\right) \mathbf{e}_{1}\right\rangle,
$$

with

$$
\begin{gathered}
\phi_{1}\left(u^{6} \mathbf{e}_{1}\right)=\mathbf{e}_{1}, \quad \phi_{1}\left(u^{6} \mathbf{e}_{1}^{\prime}+\left(c u^{2}+c^{\prime} u^{6}\right) \mathbf{e}_{1}\right)=\mathbf{e}_{1}^{\prime}, \\
N\left(\mathbf{e}_{1}\right)=0, \quad N\left(\mathbf{e}_{1}^{\prime}\right)=-c u^{24} \mathbf{e}_{1}
\end{gathered}
$$

and descent data

$$
\begin{gathered}
\widehat{\gamma}_{4}\left(\mathbf{e}_{1}\right)=-\sqrt{-1} \mathbf{e}_{1}, \quad \widehat{\gamma}_{4}\left(\mathbf{e}_{1}^{\prime}\right)=-\sqrt{-1} \mathbf{e}_{1}^{\prime}, \\
\widehat{\gamma_{3}^{ \pm 1}}\left(\mathbf{e}_{1}\right)=\mathbf{e}_{1}, \widehat{\gamma_{3}^{ \pm 1}}\left(\mathbf{e}_{1}^{\prime}\right)=\mathbf{e}_{1}^{\prime}+c\left( \pm u^{6} \pm u^{18}-u^{24} \pm u^{30}\right) \mathbf{e}_{1} .
\end{gathered}
$$

The classes in $\operatorname{Ext}_{\mathbf{F}_{3}\left[G_{3}\right]}^{1}(1,1)$ which split over an unramified extension of $\mathbf{Q}_{3}$ correspond to the pairs with $c=0$.

(2) The map of groups

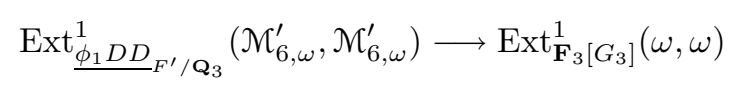

is an isomorphism.

Explicitly, the group $\operatorname{Ext}_{\phi_{1} D D}^{1}{\underline{F^{\prime} / \mathbf{Q}_{3}}}^{1}\left(\mathcal{M}_{6, \omega}^{\prime}, \mathcal{M}_{6, \omega}^{\prime}\right)$ is parametrised by pairs $\left(c, c^{\prime}\right) \in \mathbf{F}_{3}^{2}$ corresponding to

$$
\begin{gathered}
\mathcal{M}=\left(\mathbf{F}_{3}[u] / u^{36}\right) \mathbf{e}_{\omega} \oplus\left(\mathbf{F}_{3}[u] / u^{36}\right) \mathbf{e}_{\omega}^{\prime}, \mathcal{M}_{1}=\left\langle u^{6} \mathbf{e}_{\omega}, u^{6} \mathbf{e}_{\omega}^{\prime}+\left(c u^{2}+c^{\prime} u^{6}\right) \mathbf{e}_{\omega}\right\rangle \\
\text { with } \\
\phi_{1}\left(u^{6} \mathbf{e}_{\omega}\right)=\mathbf{e}_{\omega}, \phi_{1}\left(u^{6} \mathbf{e}_{\omega}^{\prime}+\left(c u^{2}+c^{\prime} u^{6}\right) \mathbf{e}_{\omega}\right)=\mathbf{e}_{\omega}^{\prime} \\
N\left(\mathbf{e}_{\omega}\right)=0, \quad N\left(\mathbf{e}_{\omega}^{\prime}\right)=-c u^{24} \mathbf{e}_{\omega}
\end{gathered}
$$


and descent data

$$
\begin{gathered}
\widehat{\gamma}_{4}\left(\mathbf{e}_{\omega}\right)=\sqrt{-1} \mathbf{e}_{\omega}, \widehat{\gamma}_{4}\left(\mathbf{e}_{\omega}^{\prime}\right)=\sqrt{-1} \mathbf{e}_{\omega}^{\prime}, \\
\widehat{\gamma_{3}^{ \pm 1}}\left(\mathbf{e}_{\omega}\right)=\mathbf{e}_{\omega}, \widehat{\gamma_{3}^{ \pm 1}}\left(\mathbf{e}_{\omega}^{\prime}\right)=\mathbf{e}_{\omega}^{\prime}+c\left( \pm u^{6} \pm u^{18}-u^{24} \pm u^{30}\right) \mathbf{e}_{\omega} .
\end{gathered}
$$

The classes in $\operatorname{Ext}_{\mathbf{F}_{3}\left[G_{3}\right]}^{1}(\omega, \omega)$ which split over an unramified extension of $\mathbf{Q}_{3}$ correspond to the pairs with $c=0$.

Proof. We treat the first part of the lemma; replacing $\sqrt{-1}$ with $-\sqrt{-1}$ throughout gives the proof of the second part.

As usual, we can find an ordered $\mathbf{F}_{3}[u] / u^{36}$-basis $\mathbf{e}_{1}, \mathbf{e}_{1}^{\prime}$ of $\mathcal{M}$ so that

$$
\mathcal{M}_{1}=\left\langle u^{6} \mathbf{e}_{1}, u^{6} \mathbf{e}_{1}^{\prime}+h \mathbf{e}_{1}\right\rangle, \quad \phi_{1}\left(u^{6} \mathbf{e}_{1}\right)=\mathbf{e}_{1}, \quad \phi_{1}\left(u^{6} \mathbf{e}_{1}^{\prime}+h \mathbf{e}_{1}\right)=\mathbf{e}_{1}^{\prime},
$$

and $\widehat{\gamma}_{4}\left(\mathbf{e}_{1}\right)=-\sqrt{-1} \mathbf{e}_{1}, \widehat{\gamma}_{4}\left(\mathbf{e}_{1}^{\prime}\right)=-\sqrt{-1} \mathbf{e}_{1}^{\prime}$. The conditions $\widehat{\gamma}_{4}\left(\mathcal{M}_{1}^{\prime}\right) \subseteq \mathcal{M}_{1}^{\prime}$ and $\widehat{\gamma}_{4} \circ \phi_{1}^{\prime}=\phi_{1}^{\prime} \circ \widehat{\gamma}_{4}$ on $\mathcal{M}_{1}^{\prime}$ amount to

$$
h(u) \equiv-h(-\sqrt{-1} u) \bmod u^{18}
$$

Since $\left\{u^{6} t-u^{6} t^{3} \mid t \in \mathbf{F}_{3}[u] / u^{36}\right\}$ consists of multiples of $u^{7}$, we can change the choice of $\mathbf{e}_{1}^{\prime}$ so that

$$
h=c u^{2}+c^{\prime} u^{6}
$$

for some $c, c^{\prime} \in \mathbf{F}_{3}$, where we may a priori lose the diagonal form of $\widehat{\gamma}_{4}$. But the same kind of calculation as in Lemma 9.3.3 shows $\widehat{\gamma}_{4}\left(\mathbf{e}_{1}^{\prime}\right)=-\sqrt{-1} \mathbf{e}_{1}^{\prime}+a \sqrt{-1} \mathbf{e}_{1}$ for some $a \in \mathbf{F}_{3}$, so the condition $\widehat{\gamma}_{4}^{4}=1$ forces $a=0$ (i.e. $\widehat{\gamma}_{4}$ still has diagonal action).

It is straightforward to compute the asserted formula for $N$, and then the wild descent data can be computed exactly as in our previous computations of wild descent data; this yields the formulas

$$
\widehat{\gamma_{3}^{ \pm 1}}\left(\mathbf{e}_{1}\right)=\mathbf{e}_{1}, \widehat{\gamma_{3}^{ \pm 1}}\left(\mathbf{e}_{1}^{\prime}\right)=\mathbf{e}_{1}^{\prime}+\left(c_{ \pm 1}+c\left( \pm u^{6} \pm u^{18}-u^{24} \pm u^{30}\right)\right) \mathbf{e}_{1},
$$

where $c_{ \pm 1} \in \mathbf{F}_{3}$. Modulo $u$, the linear action of $\gamma_{3}^{ \pm 1} \gamma_{4} \gamma_{3}^{ \pm 1} \gamma_{4}^{3}$ sends $\mathbf{e}_{1}^{\prime}$ to $\mathbf{e}_{1}^{\prime}-c_{ \pm 1} \mathbf{e}_{1}$, but $\gamma_{3}^{ \pm 1} \gamma_{4} \gamma_{3}^{ \pm 1} \gamma_{4}^{3}=1$, so $c_{\epsilon}=0$ for $\epsilon= \pm 1$. Thus, we obtain the asserted list of possibilities. The well-definedness of these examples follows from Lemma 5.2 .2 and Corollary 5.6.2.

It is easy to see that there is a non-zero map $\overline{\mathbf{F}}_{3} \otimes_{\mathbf{F}_{3}} \mathcal{M}(0,1) \rightarrow \overline{\mathbf{F}}_{3} \otimes_{\mathbf{F}_{3}} \mathcal{M}$ if and only if $c=0$, in which case such non-zero maps are precisely those induced by

$$
\mathbf{e} \longmapsto a u^{9} \mathbf{e}_{1}+u^{3}\left(u^{6} \mathbf{e}_{1}^{\prime}+c^{\prime} u^{6} \mathbf{e}_{1}\right)
$$

where $a \in \overline{\mathbf{F}}_{3}$ satisfies $a^{3}=a+c^{\prime}$. The verification that $c=c^{\prime}=0$ corresponds to being in the kernel of our map of $\mathrm{Ext}^{1}{ }^{1}$ s is now clear, since $X^{3}=X+c^{\prime}$ has a solution in $\mathbf{F}_{3}$ if and only if $c^{\prime}=0$.

9.4. Completion of the proof of Theorem 4.6.1, Everything in Theorem 4.6.1 is now clear except for the third assertion, which we now prove. Let $\left(\mathcal{G}^{\prime},\{[g]\}\right)$ be as in the third part of that theorem. We may suppose that $\mathcal{G}^{\prime}=\mathcal{G} \times{ }_{\mathcal{O}_{F}} \mathcal{O}_{F^{\prime}}$ for some $\mathcal{G}_{/ \mathcal{O}_{F}}$. The filtration on $\bar{\rho} \otimes_{\mathbf{F}_{3}} k$ gives a filtration

$$
(0) \longrightarrow \mathcal{S}_{\omega} \longrightarrow \mathcal{G} \longrightarrow \mathcal{G}_{1} \longrightarrow(0)
$$

which is compatible with the descent data over $\mathbf{Q}_{3}$. According to Lemma 5.2 .3 we have $\mathcal{M}_{\pi}\left(\mathcal{G}_{\omega}\right) \cong \mathcal{M}\left(k ; r_{\omega}, f_{\omega}\right)$ and $\mathcal{M}_{\pi}\left(\mathcal{G}_{1}\right) \cong \mathcal{M}\left(k ; r_{1}, f_{1}\right)$ for some $0 \leq r_{1}, r_{\omega} \leq 12$ and some $f_{1}, f_{\omega} \in k[u] / u^{36}$. We will let $\chi$ denote either 1 or $\omega$. In particular $\mathcal{M}_{\pi}\left(\mathcal{G}_{\chi}\right)_{1}=u^{r} \chi \mathcal{M}_{\pi}\left(\mathfrak{G}_{\chi}\right)$ for $\chi=1, \omega$. From this one can conclude that if $\mathcal{H}$ is 
a subquotient of $\mathcal{G}_{\chi}$, then $\mathcal{M}_{\pi}(\mathcal{H})_{1}=u^{r_{\chi}} \mathcal{M}_{\pi}(\mathcal{H})$. Quite generally, for any Breuil module $\mathcal{M}$ over $\mathcal{O}_{F}$ with $\mathcal{M}_{1}=u^{r} \mathcal{M}$ and any short exact sequence of Breuil modules

$$
0 \longrightarrow \mathcal{M}^{\prime} \longrightarrow \mathcal{M} \longrightarrow \mathcal{M}^{\prime \prime} \longrightarrow 0
$$

we must also have

$$
\mathcal{M}_{1}^{\prime}=u^{r} \mathcal{M}^{\prime}, \mathcal{M}_{1}^{\prime \prime}=u^{r} \mathcal{M}^{\prime \prime}
$$

Indeed, $\mathcal{M} \rightarrow \mathcal{M}^{\prime \prime}$ is a surjection taking $\mathcal{M}_{1}$ onto $\mathcal{M}_{1}^{\prime \prime}$, so the assertion for $\mathcal{M}^{\prime \prime}$ is clear. Since $\mathcal{M}^{\prime}$ is an $\mathbf{F}_{3}[u] / u^{36}$-module direct summand of $\mathcal{M}$ and

$$
\mathcal{M}_{1}^{\prime}=\mathcal{M}^{\prime} \cap \mathcal{M}_{1}=\mathcal{M}^{\prime} \cap u^{r} \mathcal{M},
$$

the assertion for $\mathcal{M}^{\prime}$ is likewise clear. We conclude that $\left(\mathcal{M}_{\pi}\left(\mathcal{G}_{\chi}\right)^{\prime},\{\widehat{g}\}\right)$ admits a filtration with successive quotients $\mathcal{M}_{r_{\chi}, \chi}^{\prime}$. Thus $r_{\chi} \in\{2,6,10\}$.

Consider a fixed surjection of $\mathbf{F}_{3}\left[G_{3}\right]$-modules

$$
\bar{\rho} \otimes k \longrightarrow \bar{\rho} .
$$

This gives rise to a finite flat $\mathcal{O}_{F}$-group scheme $\mathcal{H}$ with descent data on $\mathcal{H}^{\prime}=\mathcal{H} \times \mathcal{O}_{F^{\prime}}$ over $\mathbf{Q}_{3}$ corresponding to $\bar{\rho}$ and an epimorphism

$$
\mathcal{G} \longrightarrow \mathcal{H}
$$

compatible with descent data. Consider the commutative diagram

$$
\begin{array}{cccccc}
0 & \rightarrow \mathcal{M}_{\pi}\left(\mathcal{H}_{1}\right) & \rightarrow & \mathcal{M}_{\pi}(\mathcal{H}) & \rightarrow \mathcal{M}_{\pi}\left(\mathcal{H}_{\omega}\right) & \rightarrow 0 \\
& & & \downarrow & & \\
& & & & \downarrow & \\
0 & \rightarrow \mathcal{M}_{\pi}\left(\mathcal{G}_{1}\right) & \rightarrow & \mathcal{M}_{\pi}(\mathcal{G}) & \rightarrow \mathcal{M}_{\pi}\left(\mathcal{G}_{\omega}\right) & \rightarrow 0
\end{array}
$$

where the top row corresponds to the non-split filtration of $\bar{\rho}$. The middle vertical map is an isomorphism of the source onto an $\mathbf{F}_{3}[u] / u^{36}$-module direct summand of the target, so the left vertical map is as well, because an injection of $\mathbf{F}_{3}[u] / u^{36}$ into a free $\mathbf{F}_{3}[u] / u^{36}$-module must be an identification with such a direct summand (consider torsion). This forces $\mathcal{M}_{\pi}\left(\mathcal{H}_{1}\right)^{\prime}=\mathcal{M}_{r_{1}, \mathbf{1}}^{\prime}$ and so, by Proposition 9.2.1 we see that $r_{1} \neq 2$. Repeating the analogous argument applied to a submodule $\bar{\rho} \subseteq \bar{\rho} \otimes k$ one sees that $r_{\omega} \neq 10$.

Thus $\left(\mathcal{G}^{\prime},\{[g]\}\right)$ is weakly filtered by $\left\{\mathcal{G}_{s, 1}, \mathcal{G}_{r, \omega}\right\}$ for $(r, s)=(2,6),(6,10),(2,10)$ or $(6,6)$, as desired.

9.5. Completion of the proof of Theorem 4.6.3. Write $A_{N}$ for $\mathbf{F}_{3}[[T]] /\left(T^{N}\right)$. For $(r, s)=(2,6),(6,10)$ and $(2,10)$, we will define a Breuil module $\mathcal{M}_{N,(r, s)}$ over $\mathcal{O}_{F}$ and descent data $\{\widehat{g}\}$ for $\operatorname{Gal}\left(F^{\prime} / \mathbf{Q}_{3}\right)$ on $\mathcal{M}_{N,(r, s)}^{\prime}=\mathcal{M}_{N,(r, s)} \otimes_{\mathbf{F}_{3}} \mathbf{F}_{9}$ such that $\mathcal{M}_{N,(r, s)}$ and $\left(\mathcal{M}_{N,(r, s)}^{\prime},\{\widehat{g}\}\right)$ have compatible actions of $A_{N}$ (and $\widehat{\gamma}_{2}=1 \otimes$ Frob 3$)$. More specifically set $t=2,6$ or 8 according as $(r, s)=(2,6),(6,10)$ or $(2,10)$. Viewing $\bar{\rho}$ as an extension class, it corresponds to a particular pair $\left(c, c_{1}\right) \in \mathbf{F}_{3}^{2}$ in Proposition 9.2.1 Fix these values. Motivated by the idea of deforming the formulae in Proposition 9.2.1, we are led to define

$$
\begin{gathered}
\mathcal{M}_{N,(r, s)}=\left(A_{N}[u] / u^{36}\right) \mathbf{e}_{1} \oplus\left(A_{N}[u] / u^{36}\right) \mathbf{e}_{\omega}^{\prime}, \\
\left(\mathcal{M}_{N,(r, s)}\right)_{1}=\left\langle u^{s} \mathbf{e}_{1}, u^{r} \mathbf{e}_{\omega}^{\prime}+(c+T) u^{t} \mathbf{e}_{1}\right\rangle
\end{gathered}
$$

with

$$
\phi_{1}\left(u^{s} \mathbf{e}_{1}\right)=\mathbf{e}_{1}, \quad \phi_{1}\left(u^{r} \mathbf{e}_{\omega}^{\prime}+(c+T) u^{t} \mathbf{e}_{1}\right)=\mathbf{e}_{\omega}^{\prime}
$$


It is straightforward to check that $N \circ \phi_{1}=0$ on $\mathcal{M}_{N,(r, s)}$. We may define $A_{N}$-linear descent data on $\mathcal{M}_{N,(r, s)}^{\prime}$ by setting $\widehat{\gamma}_{2}=1 \otimes$ Frob $_{3}$ and using the following formulae.

(1) When $(r, s)=(2,6)$, set

$$
\begin{gathered}
\widehat{\gamma}_{4}\left(\mathbf{e}_{1}\right)=-\sqrt{-1} \mathbf{e}_{1}, \quad \widehat{\gamma}_{4}\left(\mathbf{e}_{\omega}^{\prime}\right)=-\sqrt{-1} \mathbf{e}_{\omega}^{\prime}, \\
\widehat{\gamma_{3}^{ \pm 1}}\left(\mathbf{e}_{1}\right)=\mathbf{e}_{1}, \widehat{\gamma_{3}^{ \pm 1}}\left(\mathbf{e}_{\omega}^{\prime}\right)=\left(1 \pm u^{18}\right)\left(\mathbf{e}_{\omega}^{\prime} \pm c_{1} u^{6} \mathbf{e}_{1}\right) .
\end{gathered}
$$

(2) When $(r, s)=(6,10)$, set

$$
\begin{gathered}
\widehat{\gamma}_{4}\left(\mathbf{e}_{1}\right)=\sqrt{-1} \mathbf{e}_{1}, \quad \widehat{\gamma}_{4}\left(\mathbf{e}_{\omega}^{\prime}\right)=\sqrt{-1} \mathbf{e}_{\omega}^{\prime}, \\
\widehat{\gamma_{3}^{ \pm 1}}\left(\mathbf{e}_{1}\right)=\left(1 \mp u^{18}\right) \mathbf{e}_{1}, \widehat{\gamma_{3}^{ \pm 1}}\left(\mathbf{e}_{\omega}^{\prime}\right)=\left(\mathbf{e}_{\omega}^{\prime} \pm c_{1} u^{6} \mathbf{e}_{1}\right) .
\end{gathered}
$$

(3) When $(r, s)=(2,10)$, set

$$
\begin{gathered}
\widehat{\gamma}_{4}\left(\mathbf{e}_{1}\right)=\sqrt{-1} \mathbf{e}_{1}, \quad \widehat{\gamma}_{4}\left(\mathbf{e}_{\omega}^{\prime}\right)=-\sqrt{-1} \mathbf{e}_{\omega}^{\prime}, \\
\widehat{\gamma_{3}^{ \pm 1}}\left(\mathbf{e}_{1}\right)=\left(1 \mp u^{18}\right) \mathbf{e}_{1}, \widehat{\gamma_{3}^{ \pm 1}}\left(\mathbf{e}_{\omega}^{\prime}\right)=\left(1 \pm u^{18}\right)\left(\mathbf{e}_{\omega}^{\prime} \pm c_{1} u^{12} \mathbf{e}_{1}\right) .
\end{gathered}
$$

It is readily checked that this defines an object of $\phi_{1} D D_{F^{\prime} / \mathbf{Q}_{3}, \mathcal{J}}$ with an action of $A_{N}$. Let $\mathcal{G}_{N,(r, s)}$ and $\left(\mathcal{G}_{N,(r, s)}^{\prime},\{[g]\}\right)$ be the corresponding finite flat $\mathcal{O}_{F}$-group scheme and finite flat $\mathcal{O}_{F^{\prime}}$-group scheme with descent data.

If $1 \leq M<N$, then we have a short exact sequence in $\underline{\phi 1}_{1} D D F^{\prime} / \mathbf{Q}_{3}, \mathcal{J}$

$$
(0) \longrightarrow \mathcal{M}_{M,(r, s)}^{\prime} \longrightarrow \mathcal{M}_{N,(r, s)}^{\prime} \longrightarrow \mathcal{M}_{N-M,(r, s)}^{\prime} \longrightarrow(0),
$$

where the first map is induced by multiplication by $T^{N-M}$. The case $M=1$ shows that

$$
\left(\mathcal{G}_{N,(r, s)},\{[g]\}\right)_{\mathbf{Q}_{3}} / T\left(\mathcal{G}_{N,(r, s)},\{\widehat{g}\}\right)_{\mathbf{Q}_{3}}
$$

corresponds to $\bar{\rho}$. Thus we get a surjection of $A_{N}\left[G_{3}\right]$-modules $A_{N}^{2} \rightarrow \mathcal{G}_{N,(r, s)}\left(\overline{\mathbf{Q}}_{3}\right)$, which must in fact be an isomorphism (count orders). Thus $\left(\mathcal{G}_{N,(r, s)},\{[g]\}\right)_{\mathbf{Q}_{3}}$ defines a deformation $\rho_{N,(r, s)}$ of $\bar{\rho}$ to $A_{N}^{2}$. For $N \geq 2$ we have $\rho_{N,(r, s)} \bmod T^{2} \cong$ $\rho_{2,(r, s)}$.

We also have an exact sequence

$$
(0) \longrightarrow \mathcal{M}_{s, 1}^{\prime} \otimes_{\mathbf{F}_{3}} A_{N} \longrightarrow\left(\mathcal{M}_{N,(r, s)}^{\prime},\{\widehat{g}\}\right) \longrightarrow \mathcal{M}_{r, \omega}^{\prime} \otimes_{\mathbf{F}_{3}} A_{N} \longrightarrow(0)
$$

in $\underline{\phi}_{1} D D F^{\prime} / \mathbf{Q}_{3}$, from which we obtain an exact sequence of $A_{N}\left[G_{3}\right]$-modules

$$
(0) \longrightarrow X_{\omega} \longrightarrow \rho_{N} \longrightarrow X_{1} \longrightarrow(0) .
$$

Note that $X_{1} \cong \mathbf{F}_{3}^{N}$ and $X_{\omega} \cong \mathbf{F}_{3}(\omega)^{N}$ as $\mathbf{F}_{3}\left[G_{3}\right]$-modules. Moreover, this sequence must split as a sequence of $A_{N}$-modules. (Use, for instance, the kernel of $\rho_{N}(\sigma)-1$ for any $\sigma \in G_{3}-G_{\mathbf{Q}_{3}(\sqrt{-3})}$.) Thus $X_{1} \cong A_{N}$ and $X_{\omega} \cong A_{N}(\omega)$ as $A_{N}\left[G_{3}\right]$-modules, so $\operatorname{det} \rho_{N}=\omega$.

Finally, we must check that the exact sequence

$$
(0) \longrightarrow \bar{\rho} \longrightarrow \rho_{2} \longrightarrow \bar{\rho} \longrightarrow(0)
$$

is not split. We have maps of Breuil modules

$$
\begin{aligned}
f_{1}: \mathcal{M}_{s, 1} & \longrightarrow \mathcal{M}_{2,(r, s)} \\
\mathbf{e} & \longmapsto \mathbf{e}_{1}
\end{aligned}
$$


and

$$
\begin{aligned}
f_{2}: \mathcal{M}_{2,(r, s)} & \longmapsto \mathcal{M}_{r, \omega} \\
\mathbf{e}_{1} & \longmapsto 0 \\
T \mathbf{e}_{1} & \longmapsto 0 \\
\mathbf{e}_{\omega}^{\prime} & \longmapsto 0 \\
T \mathbf{e}_{\omega}^{\prime} & \longmapsto \mathbf{e}
\end{aligned}
$$

compatible with descent data. These give rise to maps

$$
f_{1}^{*}: \rho_{2} \longrightarrow 1
$$

and

$$
f_{2}^{*}: \omega \longrightarrow \rho_{2}
$$

such that the composites

$$
\bar{\rho} \hookrightarrow \rho_{2} \stackrel{f_{1}^{*}}{\longrightarrow} 1
$$

and

$$
\omega \stackrel{f_{2}^{*}}{\longrightarrow} \rho_{2} \longrightarrow \bar{\rho}
$$

are non-zero.

To check that

$$
(0) \longrightarrow \bar{\rho} \longrightarrow \rho_{2} \longrightarrow \bar{\rho} \longrightarrow(0)
$$

is non-split, it suffices to check that

$$
(0) \longrightarrow \omega \longrightarrow \operatorname{ker} f_{1}^{*} / \operatorname{Im} f_{2}^{*} \longrightarrow 1 \longrightarrow(0)
$$

is non-split. However, $\operatorname{ker} f_{1}^{*} / \operatorname{Im} f_{2}^{*}$ corresponds to an object $\left(\mathcal{N}^{\prime},\{\widehat{g}\}\right)$ of

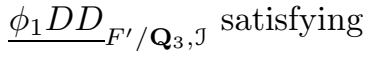

$$
\mathcal{N}^{\prime}=\left(\mathbf{F}_{9}[u] / u^{36}\right)\left(T \mathbf{e}_{1}\right) \oplus\left(\mathbf{F}_{9}[u] / u^{36}\right) \mathbf{e}_{\omega}^{\prime}, \quad \mathcal{N}_{1}^{\prime}=\left\langle u^{s}\left(T \mathbf{e}_{1}\right), u^{r} \mathbf{e}_{\omega}^{\prime}+u^{t}\left(T \mathbf{e}_{1}\right)\right\rangle
$$

with

$$
\phi_{1}\left(u^{s}\left(T \mathbf{e}_{1}\right)\right)=\left(T \mathbf{e}_{1}\right), \quad \phi_{1}\left(u^{r} \mathbf{e}_{\omega}^{\prime}+u^{t}\left(T \mathbf{e}_{1}\right)\right)=\mathbf{e}_{\omega}^{\prime} .
$$

By Lemma 5.2.2, the sequence of Breuil modules with descent data

$$
(0) \longrightarrow \mathcal{M}_{s, 1}^{\prime} \longrightarrow \mathcal{N}^{\prime} \longrightarrow \mathcal{M}_{r, \omega}^{\prime} \longrightarrow(0)
$$

is not split. This sequence recovers (9.5.1) under generic fibre descent, so by Proposition 9.2 .1

$$
(0) \longrightarrow \omega \longrightarrow \operatorname{ker} f_{1}^{*} / \operatorname{Im} f_{2}^{*} \longrightarrow 1 \longrightarrow(0)
$$

is not split. 
9.6. Completion of the proof of Theorem 4.6.2. Suppose first that $(r, s)=$ $(2,6),(6,10)$ or $(2,10)$. By Lemma 9.3.1

$$
\theta_{0}: \operatorname{Ext}_{\mathcal{S}_{i,(r, s)}}^{1}(\bar{\rho}, \bar{\rho}) \longrightarrow H^{1}\left(G_{3}, \omega\right)
$$

is the zero map. Lemma 9.3 .3 then tells us that if $r \neq 6$, then

$$
\bar{\theta}_{1}: \operatorname{Ext}_{\mathcal{S}_{i,(r, s)}}^{1}(\bar{\rho}, \bar{\rho}) \longrightarrow H^{1}\left(I_{3}, \mathbf{F}_{3}\right)
$$

is the zero map; while if $s \neq 6$, then

$$
\bar{\theta}_{\omega}: \operatorname{Ext}_{\mathcal{S}_{i,(r, s)}}^{1}(\bar{\rho}, \bar{\rho}) \longrightarrow H^{1}\left(I_{3}, \mathbf{F}_{3}\right)
$$

is the zero map. Thus Theorem 4.7.5 and hence Theorem 4.6.2, follows in these cases.

Now consider the case $(r, s)=(6,6)$. Choose $x \in H_{\mathcal{S}_{i,(6,6)}}^{1}\left(G_{3}, \operatorname{ad}^{0} \bar{\rho}\right)$. Let $\mathcal{G}$ denote the corresponding rank 81 finite flat $\mathcal{O}_{F}$-group scheme with descent data $\{[g]\}$ on $\mathcal{G}^{\prime}=\mathcal{G} \times \mathcal{O}_{F} \mathcal{O}_{F^{\prime}}$. Set $\mathcal{M}=\mathcal{M}_{\pi}(\mathcal{G})$. Let $\mathcal{H} \subset \mathcal{G}$ denote the closed subgroup scheme (with descent data) corresponding to the kernel of the map $\left(\mathcal{G}^{\prime},\{[g]\}\right) \mathbf{Q}_{3} \rightarrow$ $\bar{\rho} \rightarrow \mathbf{F}_{3}$ and let $\mathcal{N}=\mathcal{M}_{\pi}(\mathcal{H})$. Then $\mathcal{N}$ has $\mathbf{F}_{3}[u] / u^{36}$-basis $\mathbf{e}_{\omega}, \mathbf{e}_{1}^{\prime}, \mathbf{e}_{\omega}^{\prime}$ with respect to which

$$
\mathcal{N}_{1}=\left\langle u^{6} \mathbf{e}_{\omega}, u^{6} \mathbf{e}_{1}^{\prime}+\left(b+b^{\prime} u^{4}\right) \mathbf{e}_{\omega}, u^{6} \mathbf{e}_{\omega}^{\prime}+\left(c+c^{\prime} u^{4}\right) \mathbf{e}_{1}^{\prime}+f \mathbf{e}_{\omega}\right\rangle,
$$

where $b, b^{\prime}, c, c^{\prime} \in \mathbf{F}_{3}, f \in \mathbf{F}_{3}[u] / u^{36}$ and $\phi_{1}$ sends the indicated generators of $\mathcal{N}_{1}$ to $\mathbf{e}_{\omega}, \mathbf{e}_{1}^{\prime}, \mathbf{e}_{\omega}^{\prime}$ respectively. Also, the descent data has the form

$$
\widehat{\gamma}_{4}\left(\mathbf{e}_{\omega}\right)=\sqrt{-1} \mathbf{e}_{\omega}, \widehat{\gamma}_{4}\left(\mathbf{e}_{1}^{\prime}\right)=-\sqrt{-1} \mathbf{e}_{1}^{\prime}, \widehat{\gamma}_{4}\left(\mathbf{e}_{\omega}^{\prime}\right)=\sqrt{-1} \mathbf{e}_{\omega}^{\prime}+h_{\gamma_{4}}(u) \mathbf{e}_{\omega}
$$

for some $h_{\gamma_{4}} \in \mathbf{F}_{9}[u] / u^{36}$, and

$$
\begin{gathered}
\widehat{\gamma_{3}^{ \pm 1}}\left(\mathbf{e}_{\omega}\right)=\mathbf{e}_{\omega}, \widehat{\gamma_{3}^{ \pm 1}}\left(\mathbf{e}_{1}^{\prime}\right)=\mathbf{e}_{1}^{\prime}+\left( \pm b-b^{\prime}\left( \pm u^{12}+u^{30}\right)\right) \mathbf{e}_{\omega} \\
\widehat{\gamma_{3}^{ \pm 1}}\left(\mathbf{e}_{\omega}^{\prime}\right)=\mathbf{e}_{\omega}^{\prime}+\left( \pm c-c^{\prime}\left( \pm u^{12}+u^{30}\right)\right) \mathbf{e}_{1}^{\prime}+h_{ \pm 1} \mathbf{e}_{\omega}
\end{gathered}
$$

where $h_{ \pm 1} \in \mathbf{F}_{9}[u] / u^{36}$. Also, as $\bar{\rho}$ is très ramifié, we see that $c \neq 0$ by Proposition 9.2.1 and Lemma 9.3.4

The requirement that $u^{12} \mathcal{N} \subseteq \mathcal{N}_{1}$ forces $\mathcal{N}_{1}$ to contain

$$
\begin{aligned}
u^{12} \mathbf{e}_{\omega}^{\prime}= & u^{6}\left(u^{6} \mathbf{e}_{\omega}^{\prime}+\left(c+c^{\prime} u^{4}\right) \mathbf{e}_{1}^{\prime}+f \mathbf{e}_{\omega}\right)-\left(c+c^{\prime} u^{4}\right)\left(u^{6} \mathbf{e}_{1}^{\prime}+\left(b+b^{\prime} u^{4}\right) \mathbf{e}_{\omega}\right) \\
& +\left(\left(b+b^{\prime} u^{4}\right)\left(c+c^{\prime} u^{4}\right)-f u^{6}\right) \mathbf{e}_{\omega},
\end{aligned}
$$

so $\mathcal{N}_{1}$ must contain $\left(b+b^{\prime} u^{4}\right)\left(c+c^{\prime} u^{4}\right) \mathbf{e}_{\omega}$. As $c \neq 0$ we get $\left(b+b^{\prime} u^{4}\right) \mathbf{e}_{\omega} \in \mathcal{N}_{1}$, and since $\mathbf{e}_{\omega}, u^{4} \mathbf{e}_{\omega} \notin \mathcal{N}_{1}$, we must have $b=b^{\prime}=0$. We conclude that the natural map

$$
\theta_{0}: \operatorname{Ext}_{\mathcal{S}_{i,(6,6)}}^{1}(\bar{\rho}, \bar{\rho}) \longrightarrow H^{1}\left(G_{3}, \omega\right)
$$

is the zero map.

Let us further analyse $\mathcal{N}$. Replacing $\mathbf{e}_{\omega}^{\prime}$ by $\mathbf{e}_{\omega}^{\prime}+t^{3} \mathbf{e}_{\omega}$ for $t \in \mathbf{F}_{3}[u] / u^{36}$ causes $f$ to be replaced by $f-u^{6} t^{3}+u^{6} t$ and otherwise leaves our standardized form unchanged (except that $h_{\gamma_{4}}$ and $h_{ \pm 1}$ may change). Using a suitable choice of such $t$, we may assume $f$ has degree at most 6 . On the other hand,

$$
\begin{aligned}
\widehat{\gamma}_{4}\left(u^{6} \mathbf{e}_{\omega}^{\prime}+\left(c+c^{\prime} u^{4}\right) \mathbf{e}_{1}^{\prime}+f \mathbf{e}_{\omega}\right)= & -\sqrt{-1}\left(u^{6} \mathbf{e}_{\omega}^{\prime}+\left(c+c^{\prime} u^{4}\right) \mathbf{e}_{1}^{\prime}+f \mathbf{e}_{\omega}\right) \\
& +\left(\sqrt{-1}(f(u)+f(-\sqrt{-1} u))-u^{6} h_{\gamma_{4}}(u)\right) \mathbf{e}_{\omega},
\end{aligned}
$$

so $\widehat{\gamma}_{4}\left(\mathcal{N}_{1}^{\prime}\right) \subseteq \mathcal{N}_{1}^{\prime}$ if and only if

$$
f(u)+f(-\sqrt{-1} u) \equiv 0 \bmod u^{6},
$$


which forces

$$
f=a_{2} u^{2}+a_{6} u^{6}
$$

for some $a_{2}, a_{6} \in \mathbf{F}_{3}$. From the wild descent data formulae derived in the proof of Lemma 9.3.4 we also see that $h_{ \pm 1} \equiv 0 \bmod u^{6}$.

Now $\mathcal{M}$ has an ordered basis $\mathbf{e}_{1}, \mathbf{e}_{\omega}, \mathbf{e}_{1}^{\prime}, \mathbf{e}_{\omega}^{\prime}$ with respect to which

$$
\begin{aligned}
& \mathcal{M}_{1}=\left\langle u^{6} \mathbf{e}_{1}, u^{6} \mathbf{e}_{\omega}+\left(c+c^{\prime} u^{4}\right) \mathbf{e}_{1}, u^{6} \mathbf{e}_{1}^{\prime}+h \mathbf{e}_{1},\right. \\
&\left.u^{6} \mathbf{e}_{\omega}^{\prime}+\left(c+c^{\prime} u^{4}\right) \mathbf{e}_{1}^{\prime}+\left(a_{2} u^{2}+a_{6} u^{6}\right) \mathbf{e}_{\omega}+g \mathbf{e}_{1}\right\rangle,
\end{aligned}
$$

where $g, h \in \mathbf{F}_{3}[u] / u^{36}$ and $\phi_{1}$ sends the indicated generators of $\mathcal{M}_{1}$ to $\mathbf{e}_{1}, \mathbf{e}_{\omega}, \mathbf{e}_{1}^{\prime}, \mathbf{e}_{\omega}^{\prime}$. If we try to expand out $u^{12} \mathbf{e}_{\omega}^{\prime}$ as a linear combination of the indicated generators of $\mathcal{M}_{1}$, we find that

$$
u^{12} \mathbf{e}_{\omega}^{\prime} \equiv\left(\left(c+c^{\prime} u^{4}\right) h+c a_{2} u^{2}\right) \mathbf{e}_{1} \bmod \mathcal{M}_{1} .
$$

It follows that $u^{12} \mathbf{e}_{\omega}^{\prime} \in \mathcal{M}_{1}$ if and only if

$$
\left(c+c^{\prime} u^{4}\right) h+c a_{2} u^{2} \equiv 0 \bmod u^{6} .
$$

Since $\bar{\rho}$ is très ramifié, the last part of Proposition 9.2 .1 tells us that $c \neq 0$. Thus, $u^{12} \mathbf{e}_{\omega}^{\prime} \in \mathcal{M}_{1}$ if and only if $h \equiv-a_{2} u^{2} \bmod u^{6}$. We can now use Lemma 9.3 .4 to see that the wild descent data action is determined by

$$
\widehat{\gamma_{3}^{ \pm 1}}\left(\mathbf{e}_{1}\right)=\mathbf{e}_{1}, \widehat{\gamma_{3}^{ \pm 1}}\left(\mathbf{e}_{\omega}\right)=\mathbf{e}_{\omega}+\left( \pm c-c^{\prime}\left( \pm u^{12}+u^{30}\right)\right) \mathbf{e}_{1}, \widehat{\gamma_{3}^{ \pm 1}}\left(\mathbf{e}_{1}^{\prime}\right)=\mathbf{e}_{1}^{\prime}+f_{ \pm 1} \mathbf{e}_{1}
$$

(with $f_{ \pm 1} \equiv 0 \bmod u^{6}$ ), and

$$
\widehat{\gamma_{3}^{ \pm 1}}\left(\mathbf{e}_{\omega}^{\prime}\right)=\mathbf{e}_{\omega}^{\prime}+\left( \pm c-c^{\prime}\left( \pm u^{12}+u^{30}\right)\right) \mathbf{e}_{1}^{\prime}+h_{ \pm 1} \mathbf{e}_{\omega}+g_{ \pm 1} \mathbf{e}_{1}
$$

where $g_{ \pm 1} \in \mathbf{F}_{9}[u] / u^{36}$ and $h_{ \pm 1} \equiv 0 \bmod u^{6}$.

We must have

$$
\widehat{\gamma_{3}^{ \pm 1}}\left(u^{6} \mathbf{e}_{\omega}^{\prime}+\left(c+c^{\prime} u^{4}\right) \mathbf{e}_{1}^{\prime}+\left(a_{2} u^{2}+a_{6} u^{6}\right) \mathbf{e}_{\omega}+g(u) \mathbf{e}_{1}\right) \in \mathcal{M}_{1}^{\prime},
$$

and this expression is easily computed to equal

$$
\begin{aligned}
& u^{6} H_{\gamma_{3}^{+1}}^{6} \cdot\left(\mathbf{e}_{\omega}^{\prime}+\left( \pm c-c^{\prime}\left( \pm u^{12}+u^{30}\right)\right) \mathbf{e}_{1}^{\prime}+h_{ \pm 1} \mathbf{e}_{\omega}+g_{ \pm 1} \mathbf{e}_{1}\right) \\
& +\left(c+c^{\prime} u^{4} H_{\gamma_{3}^{ \pm 1}}^{4}\right)\left(\mathbf{e}_{1}^{\prime}+f_{ \pm 1} \mathbf{e}_{1}\right) \\
& \quad+\left(a_{2} u^{2} H_{\gamma_{3}^{ \pm 1}}^{2}+a_{6} u^{6} H_{\gamma_{3}^{ \pm 1}}^{6}\right)\left(\mathbf{e}_{\omega}+\left( \pm c-c^{\prime}\left( \pm u^{12}+u^{30}\right)\right) \mathbf{e}_{1}\right)+g\left(u H_{\gamma_{3}^{ \pm 1}}\right) \mathbf{e}_{1} .
\end{aligned}
$$

Remembering that $\left\langle u^{6} \mathbf{e}_{1}, u^{12} \mathcal{M}^{\prime}\right\rangle \subseteq \mathcal{M}_{1}^{\prime}$, (9.6.2) becomes

$$
u^{6}\left(\mathbf{e}_{\omega}^{\prime} \pm c \mathbf{e}_{1}^{\prime}\right)+\left(c+c^{\prime} u^{4} H_{\gamma_{3}^{ \pm 1}}^{4}\right) \mathbf{e}_{1}^{\prime}+\left(a_{2} u^{2} H_{\gamma_{3}^{ \pm 1}}^{2}+a_{6} u^{6}\right) \mathbf{e}_{\omega} \pm a_{2} c u^{2} \mathbf{e}_{1}+g(u) \mathbf{e}_{1} \in \mathcal{M}_{1}^{\prime} .
$$

Using the explicit generators of $\mathcal{M}_{1}$ given in (9.6.1) and recalling that $h \equiv-a_{2} u^{2} \bmod$ $u^{6}$, this simplifies to

$$
\pm a_{2} c u^{2} \mathbf{e}_{1} \in \mathcal{M}_{1}^{\prime} .
$$

Thus $a_{2} c u^{2}$ is divisible by $u^{6}$, so $a_{2}=0$.

The image of the class $x$ in $\operatorname{Ext}_{\mathbf{F}_{3}\left[G_{3}\right]}^{1}(\omega, \omega)$ under $\theta_{\omega}$ corresponds to a finite flat $\mathcal{O}_{F}$-group scheme with Breuil module $\mathcal{M}_{x}$ free of rank two over $\mathbf{F}_{3}[u] / u^{36}$ with basis $\mathbf{e}_{\omega}, \mathbf{e}_{\omega}^{\prime}$, and with

$$
\left(\mathcal{M}_{x}\right)_{1}=\left\langle u^{6} \mathbf{e}_{\omega}, u^{6} \mathbf{e}_{\omega}^{\prime}+a_{6} u^{6} \mathbf{e}_{\omega}\right\rangle
$$


where $\phi_{1}$ sends the indicated generators of $\left(\mathcal{M}_{x}\right)_{1}$ to $\mathbf{e}_{\omega}$ and $\mathbf{e}_{\omega}^{\prime}$ respectively. According to the proof of Lemma 9.3.4 this implies that the image of the class $x$ in $\operatorname{Ext}_{\mathbf{F}_{3}\left[G_{3}\right]}^{1}(\omega, \omega)$ is split over an unramified extension of $\mathbf{Q}_{3}$. Thus,

$$
\bar{\theta}_{\omega}: \operatorname{Ext}_{\delta_{i,(6,6)}}^{1}(\bar{\rho}, \bar{\rho}) \longrightarrow H^{1}\left(I_{3}, \mathbf{F}_{3}\right)
$$

is the zero map. This completes the proof of Theorem 4.7.5, and hence of Theorem 4.6 .2 .

\section{Corrigenda FOR CDT}

We would like to take this opportunity to record a few corrections to [CDT.

- Page 523, line -10: Insert " $K$-rational" after "For each".

- Page 532, line -6: "The semisimplicity of $\sigma_{n}$ follows from that of $\sigma_{1}$ " is false and should be deleted. This assertion was not used anywhere in the rest of the paper.

- Page 536, line 7: Replace $\mathrm{GL}_{2}(\mathbf{C})$ by $\mathrm{GL}_{2}(\mathbf{R})$.

- Page 538, line -10: Replace "of type $(S, \tau)$ " by "such that $\left.\rho\right|_{G_{\ell}}$ is of type $\tau$ and $\rho$ is of type $(S, \tau)$ ".

- Page 539, lines 18-20: Replace each $\omega_{1}$ by $\eta_{1}$ and each $\omega_{2}$ by $\eta_{2}$.

- Page 541, line 14: Replace each of the three occurrences of $\mathbf{A}$ by $\mathbf{A}^{\infty}$.

- Page 544, line -6 : "the discrete topology on $V_{p}$ " should read "the $\ell$-adic topology on $M_{p}$ ".

- Page 545, part 4 of Lemma 6.1.2: $V^{\prime}$ should be assumed to be a normal subgroup of $V$.

- Page 546, line 1: We should have noted that the key component of this argument is very similar to the main idea of [Kh].

- §6.2: There are two significant errors in this section. The assertion " $\Gamma=$ $\mathrm{SL}_{2}(\mathbf{Z}) \cap U_{S}$ satisfies the hypotheses of Theorem 6.1.1" is false and $\operatorname{Hom}\left(L_{n}, k\right)$ should be $L_{n} \otimes k$. The argument of this section can be repaired by making the following changes.

- Page 546, lines 5 and 6: Replace "Setting $S=T(\bar{\rho}) \cup\{r\}$, we find that the group $\Gamma=\mathrm{SL}_{2}(\mathbf{Z}) \cap U_{S}$ satisfies the hypotheses of Theorem 6.1.1." by "Set $S=T(\bar{\rho}) \cup\{r\} ; U_{S}^{\prime}=\prod_{p} U_{S, p}^{\prime}$ where $U_{S, p}^{\prime}=$ $U_{1}\left(p^{c_{p}}\right)$ if $p \in T(\bar{\rho})$ and $U_{S, p}^{\prime}=U_{S, p}$ otherwise; $V_{S}^{\prime}=\prod_{p} V_{S, p}^{\prime}$ where $V_{S, p}^{\prime}=U_{1}\left(p^{c_{p}}\right)$ if $p \in T(\bar{\rho})$ and $V_{S, p}^{\prime}=V_{S, p}$ otherwise; and $L_{S}^{\prime}=$ $\operatorname{Hom}_{\mathcal{O}\left[U_{S}^{\prime} / V_{S}^{\prime}\right]}\left(M_{\ell}, H^{1}\left(X_{V_{S}^{\prime}}, \mathcal{O}\right)\right)\left[I_{S}^{\prime}\right]$. Then $\Gamma=\mathrm{SL}_{2}(\mathbf{Z}) \cap U_{S}^{\prime}$ satisfies the hypotheses of Theorem 6.1.1."

- Page 546, lines 7-13: Replace $Y_{S}$ by $Y_{U_{S}^{\prime}}, \operatorname{Hom}\left(L_{n}, k\right)$ by $L_{n} \otimes k, M_{S}$ by $M_{\ell}, \mathcal{F}_{S}$ by $\mathcal{F}_{\mathrm{Hom}_{\mathcal{O}}\left(M_{\ell}, \mathcal{O}\right)}$ and $L_{S}$ by $L_{S}^{\prime}$.

- Page 546, line 13: Replace "and $\mathcal{N}_{S}$ is non-empty." by ". Using the fact that Lemma 5.1.1 holds with $U_{S}^{\prime}$ replacing $U_{S}$ and $\sigma_{\ell}$ replacing $\sigma_{S}$ and the discussion on page 541 we conclude that $\mathcal{N}_{S}$ is non-empty."

- Page 549, line -15: Replace $U_{\left\{r, r^{\prime}\right\}, p}$ by $U_{\{r\}, p}$.

- Page 549, line -11: Replace $U_{S}^{\prime} / U_{0, S}^{\prime}$ by $V_{0} / V_{1}$.

- Page 552, line 4: The assertion is false in the case $\ell \geq 5$. It can be corrected by adding "and $j(E) \not \equiv 1728 \bmod \ell$ (which is true if, for instance, $E$ has potentially supersingular reduction and $\ell \equiv 1 \bmod 4$ )" after "if $\ell \geq 5$ ".

- Page 554, line 11: Replace " $j_{E} \in$ " by " $E$ is isogenous to an elliptic curve with $j$-invariant in the set". 
- Page 554, line 11: Replace $5(29)^{3} / 2^{5}$ by $-5(29)^{3} / 2^{5}$.

- Page 554, line 17: Replace the parenthetical comment "(and $\left.j=5(29)^{3} / 2^{5}\right)$ " by "(and isogenous to one with $j$-invariant $\left.-5(29)^{3} / 2^{5}\right)$ ".

- Page 554, line -5 : Replace $p$ by $q$ and $q$ by $p$.

\section{ACKNOWLEDGEMENTS}

We would like to thank Bas Edixhoven, Rene Schoof, Tom Weston and the referee for corrections and improvements to preliminary versions of this paper; Barry Mazur for helpful conversations; and David Pollack for help with computer calculations which we made in an earlier attempt to compute some of these local deformation rings. We are grateful to the Harvard University Clay fund for supporting the first author during a key visit paid to Harvard. The second author is grateful to the Institute for Advanced Study for its stimulating environment and the University of Münster for its hospitality. The third author is grateful to Harvard University and the Université de Paris Sud for their hospitality. The fourth author is grateful to the University of California at Berkeley for its hospitality and to the Miller Institute for Basic Science for its support.

\section{REFERENCES}

[BBM] P. Berthelot, L. Breen, W. Messing, Théorie de Dieudonné cristalline II, Lecture Notes in Mathematics 930, Springer-Verlag, Berlin, 1982. MR 85k:14023

[Br1] C. Breuil, Schémas en groupes et modules filtrés, C. R. Acad. Sci. Paris 328 (1999), 93-97. MR 99m:14086

[Br2] C. Breuil, Groupes p-divisibles, groupes finis et modules filtrés, Annals of Math. 152 (2000), 489-549. CMP 2001:06

[Ca1] H. Carayol, Sur les représentations $\ell$-adiques associées aux formes modulaires de Hilbert, Ann. Sci. Éc. Norm. Sup. 19 (1986), 409-468. MR 89c:11083

[Ca2] H. Carayol, Sur les représentations galoisiennes modulo $\ell$ attachées aux formes modulaires, Duke Math. J. 59 (1989), 785-801. MR 91b:11058

[Co] B. Conrad, Ramified deformation problems, Duke Math. J. 97 (1999), 439-514. MR 2000h:11055

[CDT] B. Conrad, F. Diamond, R. Taylor, Modularity of certain potentially Barsotti-Tate Galois representations, J. Amer. Math. Soc. 12 (1999), 521-567. MR 99i:11037

[deJ] A.J. de Jong, Finite locally free group schemes in characteristic $p$ and Dieudonné modules, Inv. Math. 114 (1993), 89-138. MR 94j:14043

[De] P. Deligne, Formes modulaires et représentations $\ell$-adiques, in: Lecture Notes in Math. 179, Springer-Verlag, 1971, pp. 139-172.

[DS] P. Deligne, J.-P. Serre, Formes modulaires de poids 1, Ann. Sci. Ec. Norm. Sup. 7 (1974), 507-530. MR 52:284

[Di1] F. Diamond, The refined conjecture of Serre, in Elliptic Curves, Modular Forms and Fermat's Last Theorem (Hong Kong, 1993), International Press, 1995, pp. 22-37. MR 97b:11065

[Di2] F. Diamond, On deformation rings and Hecke rings, Ann. Math. 144 (1996), 137-166. MR 97d:11172

[DT] F. Diamond, R. Taylor, Lifting modular mod $\ell$ representations, Duke Math. J. 74 (1994), 253-269. MR 95e:11052

[E] T. Ekedahl, An effective version of Hilbert's irreducibility theorem, in "Séminaire de Théorie des Nombres, Paris 1988-89", Birkhäuser, 1990. MR 92f:14018

[EGA] A. Grothendieck, Éléments de Géométrie Algébrique $\mathrm{IV}_{4}$, Publ. Math. IHES 32, 1966-7. MR 39:220

[Fa] G. Faltings, Endlichkeitssätze für abelsche Varietäten über Zahlkörpern, Inv. Math. $\mathbf{7 3}$ (1983), 349-366. MR 85g:11026a; erratum MR 85g:11026b 
[FM] J.-M. Fontaine, B. Mazur, Geometric Galois representations, in Elliptic Curves, Modular Forms and Fermat's Last Theorem (Hong Kong, 1993), International Press, 1995, pp. 41-78. MR 96h:11049

[G] P. Gérardin, Facteurs locaux des algèbres simples de rang 4. I, in Groupes Réductifs et Formes Automorphes, I (Paris, 1976-77) Univ. Paris VII, 1978, pp. 37-77. MR 84f:22023

[Kh] C. Khare, A local analysis of congruences in the $(p, p)$ case: Part II, Invent. Math. 143 (2001), no. 1, 129-155. CMP 2001:06

[Kl] F. Klein, Vorlesungen über das Ikosaeder und die Auflösung der Gleichungen vom fünften Grade, Teubner, 1884.

[L] R.P. Langlands, Base Change for GL(2), Annals of Math. Studies 96, Princeton Univ. Press, Princeton, 1980. MR 82a:10032

[Man] J. Manoharmayum, Pairs of mod 3 and mod 5 representations arising from elliptic curves, Math. Res. Lett. 6 (1999), 735-754. MR 2000m:11045

[Maz] B. Mazur, Number theory as gadfly, Amer. Math. Monthly 98 (1991), 593-610. MR 92f: 11077

[Ra] M. Raynaud, Schémas en groupes de type $(p, p, \ldots, p)$, Bull. Soc. Math. France 102 (1974), 241-280. MR 54:7488

[Ri] K. Ribet, The $\ell$-adic representations attached to an eigenform with Nebentypus: a survey, in: Lecture Notes in Math. 601, Springer-Verlag, 1977, pp. 17-52. MR 56:11907

[SBT] N. Shepherd-Barron, R. Taylor, Mod 2 and $\bmod 5$ icosahedral representations, J. Amer. Math. Soc. 10 (1997), 283-298. MR 97h:11060

[Se1] J.-P. Serre, Local Fields, Springer-Verlag, 1979. MR 82e:12016

[Se2] J.-P. Serre, Sur les représentations modulaires de degré 2 de Gal $(\overline{\mathbf{Q}} / \mathbf{Q})$, Duke Math. J. 54 (1987), 179-230. MR 88g:11022

[Sh1] G. Shimura, On elliptic curves with complex multiplication as factors of the Jacobians of modular function fields, Nagoya Math. J. 43 (1971), 199-208. MR 45:5111

[Sh2] G. Shimura, Introduction to the Arithmetic Theory of Automorphic Functions, Princeton Univ. Press, Princeton, 1971. MR 47:3318

[Sh3] G. Shimura, Response to 1996 Steele Prize, Notices of the AMS 43 (1996), 1344-1347.

$[\mathrm{T}] \mathrm{J}$. Tate, $p$-divisible groups, in "Proceedings of a conference on local fields (Driebergen, 1966)", Springer, 1967, 158-183. MR 38:155

[TW] R. Taylor, A. Wiles, Ring-theoretic properties of certain Hecke algebras, Ann. Math. 141 (1995), 553-572. MR 96d:11072

[T] J. Tunnell, Artin's conjecture for representations of octahedral type, Bull. Amer. Math. Soc. 5 (1981), 173-175. MR 82j:12015

[We1] A. Weil, Über die Bestimmung Dirichletscher Reihen durch Funktionalgleichungen, Math. Ann. 168 (1968), 149-156. MR 34:7473

[We2] A. Weil, Scientific works. Collected papers. III (1964-1978), Springer-Verlag, 1979. MR 80k:01067c

[Wi] A. Wiles, Modular elliptic curves and Fermat's Last Theorem, Ann. Math. 141 (1995), 443-551. MR 96d:11071

Département de Mathématiques, CNRS, Université Paris-Sud, 91405 Orsay Cedex, FRANCE

E-mail address: Christophe.BREUIL@math.u-psud.fr

Department of Mathematics, Harvard University, Cambridge, Massachusetts 02138

E-mail address: bconrad@math.harvard.edu

Current address: Department of Mathematics, University of Michigan, Ann Arbor, Michigan 48109

E-mail address: bdconrad@math.1sa.umich.edu

Department of Mathematics, Brandeis University, Waltham, Massachusetts 02454

E-mail address: fdiamond@euclid.math.brandeis.edu

Department of Mathematics, Harvard University, Cambridge, Massachusetts 02138

E-mail address: rtaylor@math.harvard.edu 